\title{
Tesis Doctoral \\ Formación y Evolución \\ de Enanas Blancas Deficientes \\ en Hidrógeno
}

Autor: Lic. Jorge Alejandro Panei

Director: Dr. Leandro Gabriel Althaus

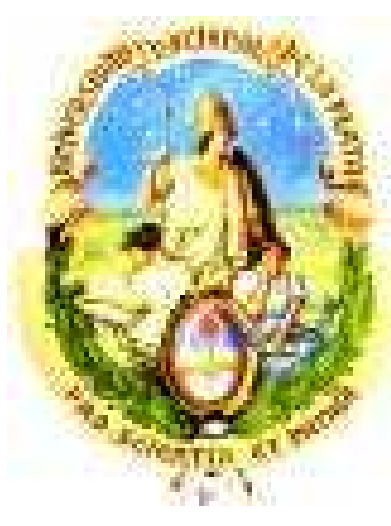

Junio de 2004

Facultad de Ciencias Astronómicas y Geofísicas

Universidad Nacional de La Plata

Paseo del Bosque S/N, (B1900FWA) La Plata, Argentina 


\section{Resumen}

Desde hace tiempo la explicación de la existencia de estrellas deficientes en hidrógeno ha sido de amplio debate. Uno de los mecanismos posibles que explica la formación de estrellas post-AGB con estas características, involucra un flash tardío de la capa de helio. Para arribar a este tipo de objetos hemos calculado la evolución previa, para estrellas de masa intermedia, desde la ZAMS hasta el recorrido a lo largo de la rama de enfriamiento de las enanas blancas, ya que es de crucial importancia tener un perfil químico realista lo cual es posible de obtener únicamente si se calcula toda la evolución de la estrella desde su formación.

Las estrellas de masa intermedia son las estrellas más abundantes en el universo y por lo tanto de fundamental importancia para su comprensión. Por estrellas de masa intermedia entendemos a aquellas que poseen una masa entre $2-2.3 M_{\odot}$ y $7-8 M_{\odot}$, las que finalizarán sus vidas como estrellas enanas blancas, estos son objetos extremadamente densos y compactos que evolucionan lentamente a expensas de su energía térmica almacenada durante su evolución previa, hasta llegar a la invisibilidad.

En particular, los aspectos vinculados a la evolución de estrellas de masa intermedia como las estrellas ricas en carbono, la formación de elementos pesados mediante los procesos de captura lenta de neutrones, la abundancia de litio, los procesos de nucleosíntesis durante la fase térmicamente pulsante, etc, constituyen temas de intensa investigación en la actualidad.

Igualmente el destino final de estas estrellas, las enanas blancas son un campo de investigación sumamente atractivo por el interés que reporta, no solamente para estas estrellas en sí mismo, sino también para numerosos campos más allá de la astrofísica estelar. Podemos mencionar algunos temas de investigación vinculados a ellas:

- El uso de enanas blancas como cronómetros estelares independientes para determinar edades de cúmulos globulares. El desarrollo de telescopios de nueva generación ha permitido poder observar la secuencia de enfriamiento de las enanas blancas en cúmulos estelares cercanos. Esto ha abierto la posibilidad de utilizar modelos evolutivos de estas estrellas para estimar la edad de dichos cúmulos de manera independiente a los métodos tradicionales.

- Es bien sabido que muchas enanas blancas exhiben pulsaciones no radiales en algunas etapas de su larga evolución. En particular, la astrosismología se ha convertido, en los ultimos años, en una herramienta sumamente potente para investigar la estructura interna de estas estrellas y de sus progenitores. Más aún, recientemente la posibilidad de establecer la existencia de un núcleo cristalizado en enanas blancas pulsantes mediante su espectro pulsacional, ha captado la atención de numerosos investigadores. 
- El empleo de las enanas blancas como laboratorios ideales para el estudio de procesos físicos imposibles de reproducir en laboratorios terrestres. Las densidades centrales extremadamente elevadas convierten a estas estrellas en objetos únicos para el estudio de partículas fundamentales débilmente interactuantes tales como los neutrinos, axiones $\mathrm{y}$ variaciones de constantes fundamentales.

- La existencia de una conexión evolutiva entre las estrellas calientes PG1159 ricas en helio, carbono y oxígeno con las enanas blancas ricas en helio de tipo espectral DB a temperaturas mucho más frías, conocida como la conexión evolutiva PG1159-DB-DQ.

La presente tesis aborda algunos de los ítems mencionados. En particular nuestro interés radica en explorar los mecanismos que llevan a la formación de las enanas blancas deficientes en hidrógeno, desde un punto de vista de una descripción detallada de los procesos físicos responsables de los cambios de abundancia que tienen lugar a lo largo de toda la evolución de la estrella progenitora. En efecto la composición química interna es el punto más importante en el modelado de estas estrellas. Para ello empleamos un esquema numérico que permite calcular los cambios químicos inducidos por los mecanismos de mezcla de manera simultánea con los cambios producidos por las reacciones nucleares; teniendo en cuenta los procesos físicos que forman el núcleo de carbono-oxígeno, como la mezcla convectiva, overshooting, inestabilidad tipo Rayleigh-Taylor y difusión microscópica.

Para tal fin se han calculado modelos estelares mediante el código desarrollado íntegramente en el Observatorio de La Plata (LPCODE) y escrito en forma independiente de otros investigadores. Los modelos evolutivos fueron calculados desde la secuencia de edad cero, pasando por la fase de gigante roja, la evolución durante la fase térmicamente pulsante de la AGB, el escenario de "born again" (durante el cual la estrella vuelve hacia la región de las gigantes rojas y donde la envoltura remanente rica en hidrógeno es completamente consumida por reacciones nucleares), la etapa de nebulosa planetaria, el estado de PG1159 y calculando finalmente la rama de enfriamiento de las enanas blancas, obteniendo así modelos estelares de enanas blancas deficientes en hidrógeno, es decir de tipo espectral DB, de manera consistente con la historia evolutiva de los progenitores de estas estrellas, finalizando con modelos de estrellas DQ, remanentes estelares contaminados de carbono en su superficie.

Además del problema de la formación de las enanas blancas deficientes en hidrógeno, la presente tesis tiene como otro objetivo el cálculo de relaciones masa-radio a temperatura finita para enanas blancas de distintas composiciones químicas y masas estelares. En vista del grado de sofisticación alcanzado por las observaciones actuales, dichas relaciones masa-radio resultan de suma utilidad en la interpretación de numerosas propiedades de estas estrellas. 


\section{Índice General}

$\begin{array}{lll}\text { Resumen } & \text { I }\end{array}$

Índice General

1 Introducción $\quad 1$

2 Aspectos físicos y computacionales $\quad 6$

2.1 Descripción general del código . . . . . . . . . . . . . . . 6

2.2 Evolución química . . . . . . . . . . . . . . . . . 8

2.3 Overshooting $(\mathrm{OV}) \ldots \ldots \ldots \ldots \ldots$

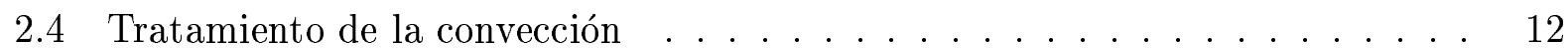

2.5 Tasa de pérdida de masa . . . . . . . . . . . . . . . 12

Parte I: Relación masa-radio $\quad 14$

3 Relaciones masa-radio $\quad 15$

3.1 Masa límite de Chandrasekhar . . . . . . . . . . . . . . . 15

3.2 Refinamientos a la teoría de Chandrasekhar: modelos de Hamada y Salpeter 15

3.3 Relaciones masa-radio para enanas blancas con diferentes composiciones

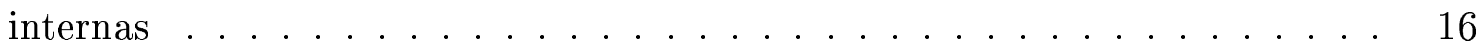

Parte II: Formación de estrellas deficientes en hidrógeno 26

4 Evolución previa a la AGB $\quad 27$

4.1 Evolución hacia la fase gigante . . . . . . . . . . . . . . 27

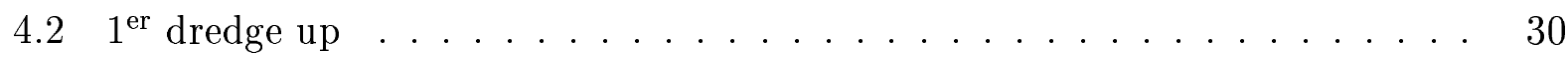

4.3 Evolución en la RGB . . . . . . . . . . . . . . . . . . . . . 30

4.3.1 Estrellas de baja masa . . . . . . . . . . . . 30

4.3.2 Estrellas de masa intermedia . . . . . . . . . . . . 32

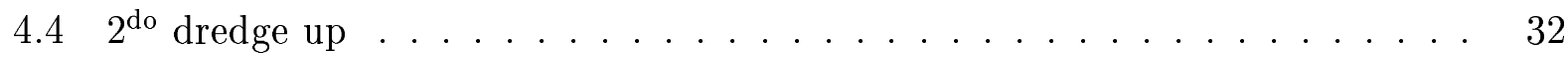

4.5 Evolución durante la AGB temprana . . . . . . . . . . . . . . 35

5 Evolución durante la AGB $\quad 37$

5.1 Pulsos térmicos y $3^{\text {er }}$ mezclado convectivo externo . . . . . . . . . 37

5.2 El rol del overshooting . . . . . . . . . . . . . . . . . 45

5.3 Hot Bottom Burning $(\mathrm{HBB}) \ldots \ldots \ldots 46$

5.4 La barrera de entropía . . . . . . . . . . . . . . . . . . 49 
5.4.1 La barrera de entropía previene el contacto entre las regiones de la capa y la envoltura convectiva . . . . . . . . . . . 52

5.4 .2 El MCE y el carbono estelar . . . . . . . . . . . . . . . . . . . . . . . . . . . . . 53

5.4.3 El por qué del MCE y la entropía . . . . . . . . . . 55

6 Evolución más allá de la AGB $\quad 56$

6.1 Pérdida de masa . . . . . . . . . . . . . . . 56

6.2 Pulsos térmicos finales . . . . . . . . . . . . . . 57

6.3 Pulso térmico final de la AGB (AFTP) . . . . . . . . . . . . . 58

6.4 Pulso térmico tardío $(\mathrm{LTP}) \ldots \ldots \ldots \ldots$. . . . . . . . . . . 59

6.5 Pulso térmico muy tardío $(\mathrm{VLTP}) \ldots \ldots \ldots \ldots$. . . . . . . . . . . . . . . . . . .

$\begin{array}{lll}7 & \text { La baja abundancia de hidrógeno } & 68\end{array}$

7.1 Abell 30 y Abell $78 \ldots \ldots \ldots$. . . . . . . . . . . . . . 82

7.1.1 Dilución y quema de hidrógeno . . . . . . . . . . 83

7.1.2 Dilución convectiva superficial . . . . . . . . . . . 84

7.1.3 Eyección debida al viento . . . . . . . . . . . . . . 84

7.1.4 Mecanismos posibles para explicar el nitrógeno superficial . . . . . . 85

8 Escenarios born again $\quad \mathbf{8 6}$

8.1 Objetos Sakurai: otra evidencia observacional de born again . . . . . . 86

8.1.1 La historia evolutiva de un objeto Sakurai: V4334 Sgr . . . . . . . . . 86

8.1.2 ¿Cómo se reproduce un modelo de un objeto Sakurai? . . . . . . . . 88

8.1.3 Comparación con las observaciones . . . . . . . . . . 89

8.2 Tratamiento de la convección _. . . . . . . . . . . . . . . 90

9 Las estrellas PG1159 94

9.1 Generalidades . . . . . . . . . . . . . . . . . 94

9.2 Evolución de las PG1159 . . . . . . . . . . . . . . . 96

10 Aspectos generales de las enanas blancas $\quad 99$

10.1 ¿Cuáles estrellas se convierten en enanas blancas? . . . . . . . . . . . . 99

10.2 Aspectos observacionales . . . . . . . . . . . . . . . . . . . . . . . . . . . . . . . . . . . . . . . . . . . . . . . . .

10.3 Aspectos estadísticos . . . . . . . . . . . . . . . . . 100

10.4 Clasificación espectral _ . . . . . . . . . . . . . . . . . 101

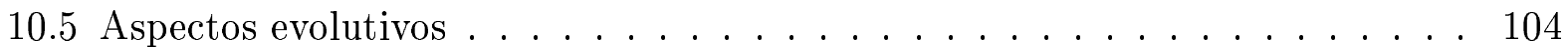

10.6 Enanas blancas variables: bandas de inestabilidad . . . . . . . . . . . 105

10.6.1 Estrellas pre-enanas blancas variables . . . . . . . . 105 
10.6.2 Estrellas variables DBV (o V4777 Her) . . . . . . . . . . . . 107

10.6.3 Estrellas variables DAV (o ZZ Ceti) . . . . . . . . . . . . . 107

11 Evolución de las enanas blancas $\quad 109$

11.1 Primeras etapas de la rama de enfriamiento . . . . . . . . . . . . . 109

11.2 Enanas blancas DO . . . . . . . . . . . . . . . . . . . . 109

11.3 Enanas blancas DB . . . . . . . . . . . . . . . . . . . 113

11.4 Enanas blancas DQ . . . . . . . . . . . . . . . . . . . . . . . . . . . . . . . . . . .

11.5 Una enana blanca DB: GD358 . . . . . . . . . . . . 116

$\begin{array}{lr}12 \text { Conclusiones } & 118\end{array}$

13 Apéndice A: Conceptos astrofísicos $\quad 124$

13.1 Ecuación de equilibrio hidrostático . . . . . . . . . . . . . . . . . 124

13.2 Transporte de energía por radiación, conducción y convección . . . . . . 125

13.2.1 Transporte de energía radiativa . . . . . . . . . . . . 125

13.2.2 Transporte de energía conductiva . . . . . . . . . . . . 127

13.2 .3 Gradiente radiativo . . . . . . . . . . . . . . . . 127

13.2 .4 Gradiente adiabático . . . . . . . . . . . . . . . . 128

13.3 Inestabilidad dinámica . . . . . . . . . . . . . . . . . . . . . . . 129

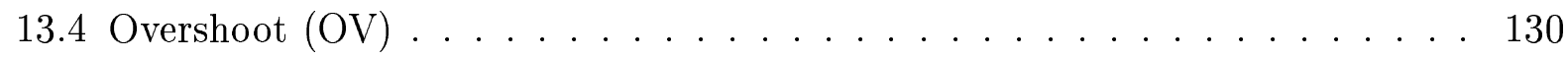

13.5 Semiconvección . . . . . . . . . . . . . . . . . 130

13.6 Peso molecular medio y presión de radiación . . . . . . . . . . . . . 131

13.7 Física de las enanas blancas . . . . . . . . . . . . . . . . . 134

13.7.1 Ecuación de estado para un gas ideal de electrones . . . . . . . . 134

13.7.2 La ecuación de Chandrasekhar . . . . . . . . . . . . . . . 138

13.7.3 Modelos estelares simples, relación masa-radio y el límite de Chandrasekhar . . . . . . . . . . . . . . . . . 139

14 Apéndice B: Glosario 143

15 Apéndice C: Tabla de símbolos $\quad 145$

$\begin{array}{lr}16 \text { Apéndice D: Tabla de unidades } & 146\end{array}$

17 Apéndice E: Abundancias Solares y Reacciones Nucleares 147

17.1 Abundancias solares . . . . . . . . . . . . . . . . . . . . 147

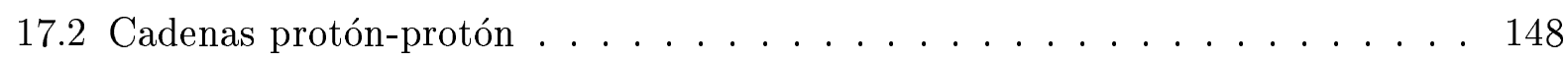

17.3 Ciclo CNOF . . . . . . . . . . . . . . . . . . . . . . 149 
17.4 Quema de helio . . . . . . . . . . . . . . . 150

17.5 Quema de carbono y oxígeno . . . . . . . . . . . . . . 151

17.6 Fotodesintegración del neón . . . . . . . . . . . . . . 152

$\begin{array}{lr}\text { Bibliografía } & 153\end{array}$ 


\section{Índice de Figuras}

1 Relación masa-radio para enanas blancas con un núcleo de helio. Las líneas llenas y punteadas corresponden a los casos de objetos con una envoltura de hidrógeno puro de $3 \times 10^{-4} M_{*}$ y a los modelos sin esa envoltura de hidrógeno, respectivamente. Las líneas de trazos representan la relación masa-radio de $H$-S para modelos homogéneos de helio. Los modelos de temperatura finita están ordenados desde abajo hacia arriba con el aumento de temperatura $T_{\text {eff }}$ correspondiente a (en $\left.10^{3} \mathrm{~K}\right)$ 4, 8, 12, 16 y 20.

2 Relación masa-radio para enanas blancas con un núcleo de carbono rodeado por una envoltura de helio con un espesor de $10^{-2} M_{*}$. Las lineas llenas y las punteadas tienen el mismo significado que en la fig. (1) pero para el caso de la envoltura de hidrógeno asumimos una masa de $10^{-5} M_{*}$. Las líneas de trazos corresponden a la relación masa-radio de H-S para modelos homogéneos de carbono. Hemos incluido en esta figura los valores correspondientes a $T_{\text {eff }}\left(\right.$ en $\left.10^{3} \mathrm{~K}\right)$ de $5,15,25,35,45,55$, 70, 85, 100, 115, 130 y 145. Hemos incluido datos observacionales con sus barras de error de las enanas blancas que se indican. Por completitud hemos extendido los cálculos hasta una masa de $0.3 M_{\odot}$. . . . . . . . . . . . . . . . 21

$3 \quad$ Igual que en la fig. (2) pero para un núcleo de oxígeno. . . . . . . . . . . . . 22

$4 \quad$ Igual que en la fig. (2) pero para un núcleo de silicio. . . . . . . . . . . . . .

$5 \quad$ Igual que en la fig. (2) pero para un núcleo de hierro. Notar que en este caso los modelos resultan con radios estelares mucho menores que para las demás composiciones químicas centrales. . . . . . . . . . . . . . . . .

$6 \quad$ Interior estelar de una estrella de $M_{Z A M S}=5 M_{\odot}$, se representan los distintos transportes de energía, y las quemas de hidrógeno y helio en el interior. En ambas regiones de quema se tiene en cuenta la generación de energía nuclear $\left(\varepsilon_{H} y \varepsilon_{H e}\right)$, cuando excede a $10^{3} \mathrm{erg}^{-1} \mathrm{~s}^{-1}$. Obsérvese la disminución de la masa estelar total (la línea llena que comienza en $M / M_{\odot}=5$ ), en este caso la pérdida de masa la iniciamos, en el código de evolución, cuando la estrella trepa por la rama gigante.

$7 \quad$ Trayectoria evolutiva de una estrella de $M_{Z A M S}=5 M_{\odot}$, diagrama $H-R$ y evolución de su región central. A la derecha graficamos la rama gigante en forma ampliada, para ver con más detalle los puntos dados en la tabla [(2), sec. (4.1)]. . . . . . . .

8 Interior estelar de una estrella de $M_{Z A M S}=5 M_{\odot}$, durante el $1^{\text {er }}$ "dredge up". Representamos los distintos transportes de energía y la quema de hidrógeno y helio en el interior, para el caso en que las generaciones de energía nuclear $\varepsilon_{H}$ y $\varepsilon_{H e}$ exceden a $10^{3} \operatorname{erg}^{-1} s^{-1} \ldots \ldots \ldots \ldots \ldots$ 
$9 \quad$ Interior estelar de una estrella de $M_{Z A M S}=5 M_{\odot}$, durante el $2^{\text {do }}$ "dredge up", para

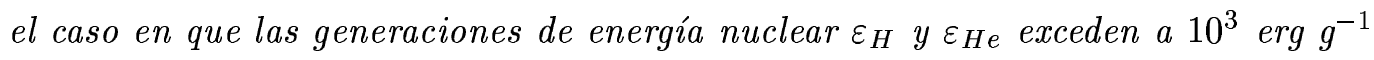
$s^{-1} \ldots \ldots \ldots \ldots \ldots \ldots \ldots \ldots \ldots$

10 Cambios de abundancias superficiales durante el $1^{\text {er }}$ y $2^{\text {do }}$ "dredge up", para una estrella $M_{Z A M S}=5 M_{\odot}$. Para las curvas representadas a la izquierda, la línea de segmentos indica el comienzo del $1^{\text {er }}$ "dredge up" (punto C) y la correspondiente de punto y segmentos el fin del mismo (punto D). En las curvas de la derecha las líneas de segmentos y de segmentos y puntos tienen el mismo significado anterior, pero para el $2^{\text {do }}$ "dredge up" (puntos $F$ y $H$ ). La línea de puntos es la que corresponde al punto $G$, que es durante el momento en que se va acentuando el $2^{\text {do }}$ "dredge up'. .

11 Luminosidad superficial, temperatura efectiva, la luminosidad debido a la quema de hidrógeno por el ciclo p-p y CNO, y la quema de helio; para una estrella de $M_{Z A M S}=2.5 M_{\odot} \ldots \ldots \ldots \ldots \ldots \ldots$

12 Interior de una estrella con una masa de $M_{Z A M S}=2.5 M_{\odot}$, durante el $9^{\text {no }}$, $10^{\text {mo }}$ y $11^{\text {mo }}$ pulso. Se indican distintos tipos de transporte y también la luminosidad integrada debida a la quema de helio. En la figura se ha agregado en qué lugar debajo de la envoltura convectiva, se forma la capa conductora de los pulsos. Notar que esta es una región diminuta de la estrella, ya que la misma es $\Delta m_{r} \simeq 0.02 M_{\odot}$.

13 Interior de una estrella con una masa de $M_{Z A M S}=2.5 M_{\odot}$, durante el $9^{\text {no }}, 10^{\text {mo }}$ y $11^{\text {mo }}$ pulso. Se muestra la región correspondiente donde se encuentra la capa convectiva. Se indican distintos tipos de transporte y también la quema de hidrógeno y helio. Se ha considerado liberación de energía cuando $\varepsilon_{H}>10^{3} \mathrm{erg}^{-1} s^{-1} y$ $\varepsilon_{H e}>3 \times 10^{6} \mathrm{erg}^{-1} \mathrm{~s}^{-1} \ldots \ldots \ldots \ldots \ldots$

14 Interior de una estrella con una masa de $M_{Z A M S}=2.5 M_{\odot}$, durante el $9^{\text {no }}, 10^{\text {mo }}$ y $11^{\text {mo }}$ pulso. Se muestran en forma ampliada las capas convectivas, los distintos tipos de transporte y también la quema de hidrógeno y helio. En la figura se muestra como cesa la quema de hidrógeno debido a que la capa de hidrógeno es empujada hacia afuera de la estrella hacia regiones más frías. Notar que posteriormente la envoltura convectiva avanza hacia adentro para dragar el helio, carbono y oxígeno hacia la superficie. Se ha considerado liberación de energía cuando $\varepsilon_{H}>10^{3} \mathrm{erg}^{-1} s^{-1} y$ $\varepsilon_{H e}>3 \times 10^{6} \mathrm{erg}^{-1} \mathrm{~s}^{-1} \ldots \ldots \ldots \ldots \ldots \ldots$

15 Interior de una estrella con una masa de $M_{Z A M S}=2.5 M_{\odot}$, durante el $9^{\text {no }}$ pulso. Se muestran los distintos tipos de transporte y la quema de hidrógeno y helio. Se indican las luminosidades integradas de la quema de hidrógeno por los ciclos p-p y CNO, y la correspondiente a la de helio. Se ha considerado liberación de energía cuando $\varepsilon_{H}>10^{3} \mathrm{erg}^{-1} \mathrm{~s}^{-1}$ y $\varepsilon_{H e}>3 \times 10^{6} \mathrm{erg}^{-1} \mathrm{~s}^{-1} \ldots \ldots \ldots$ 
16 Abundancias superficiales y $3^{\text {er }}$ "dredge up", para una estrella con masa $M_{Z A M S}=$ $2.5 M_{\odot}$. Se han agregado los pulsos debido a la quema de helio. Observar como a partir del $8^{v o}$ pulso se produce un enriquecimiento en la superficie estelar con helio, carbono y oxígeno, y una disminución del hidrógeno. . . . . . . . . . . . . .

17 Temperatura vs. masa para las regiones convectivas para estrellas con diferentes masas iniciales $\left(M_{Z A M S}\right)$. La región sombreada representa la base de la envoltura convectiva y los segmentos verticales son las zonas convectivas conductoras de los pulsos de la capa de helio (Blöcker et al., 1999). . . . . . . . . . . . . . . .

18 Temperatura de la base de la envoltura convectiva $\left(T_{\text {bottom }}\right)$ para modelos con masas $M_{Z A M S}=4,5$ y $6 M_{\odot}$. El fuerte aumento de $T_{\text {bottom }}$ indica la eficiencia del $H B B$. El timepo $t=0$ se refiere al máximo de $L_{H e}$ del primer pulso térmico (Blöcker et

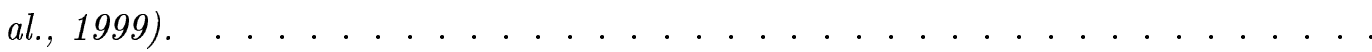

19 (a) En la figura superior izquierda mostramos, a partir de nuestros cálculos, la región interna de la estrella en donde está presente la capa conductora del $g^{n o}$ pulso. Se ha considerado para la quema de hidrógeno y helio que $\varepsilon_{H}>10^{3} \mathrm{erg}^{-1} s^{-1} y$ $\varepsilon_{H e}>3 \times 10^{6} \mathrm{erg} \mathrm{g}^{-1} \mathrm{~s}^{-1}$. Los números corresponden a un valor dado para distintas coordenadas masa. (b) En la figura superior derecha se muestra como la barrera de entropía se levanta hacia el máximo de $\varepsilon_{H e}$. (c) En la figura inferior izquierda se grafica la marcha de la temperatura con el tiempo para distintas coordenadas masas, se observa que para la capa 1 la temperatura aumenta en $\simeq 100 \times 10^{6} \mathrm{~K}$ (de 158 a 251 en $\left.10^{6} \mathrm{~K}\right)$. (d) En la figura inferior derecha tenemos densidad vs. tiempo, observar que después del pulso la densidad de la capa 1 , cae $\approx 1$ orden de magnitud $\left(d e \approx 4.4\right.$ a 0.45 en unidades de $\left.10^{4} \mathrm{~g} \mathrm{~cm}^{-3}\right)$. . . . . . . . . . . . . . .

20 (a) Para la figura superior izquierda ver los comentarios de la fig. (19). (b) En la figura superior derecha se grafica el radio en función del tiempo. Notar cómo se expande la estrella en la parte superior de la capa convectiva (capa 13) moviéndose $\approx 2.37 \times 10^{5} \mathrm{~km} \mathrm{hacia} \mathrm{afuera}\left(\approx \frac{1}{3} R_{\odot}\right.$, aumenta de 0.141 a $\left.2.511 \times 10^{10} \mathrm{~cm}\right)$. (c) En la figura inferior izquierda se representa la liberación de energía nuclear $\left(\varepsilon_{n u c}\right)$ para las capas 1, 2, y 3, estas capas corresponden a la zona en donde $\varepsilon_{n u c}$ es mayor. Se agregan también las luminosidades integradas en toda la estrella $L_{p-p}, L_{C N O} y$ $L_{H e}$. (d) En la figura inferior derecha se grafica la presión de radiación vs. tiempo.

21 Se grafica el comportamiento del interior de la estrella en una región próxima a la capa convectiva y para varias etapas evolutivas en un entorno del $9^{\text {no }}$ y $10^{\text {mo }}$ pulso. Se muestran los perfiles internos de ${ }^{1} \mathrm{H},{ }^{4} \mathrm{He} y{ }^{12} \mathrm{C}$, las regiones con overshooting y convectivas pertenecientes a la envoltura y a la capa conductora de cada pulso. . . 
22 AFTP: se muestra la evolución de modelos post-AGB con masas $\left(M_{Z A M S} / M_{\odot}\right.$, $\left.M_{W D} / M_{\odot}\right)=(3,0.605),(3,0.625)$ y $(5,0.836)$. Las marcas indican el tiempo en $10^{3}$ años. . . . . . . . . . . . . . . . . .

23 LTP: se muestra la evolución de un modelo post-AGB con masas $\left(M_{Z A M S}, M_{W D}\right)$ $=\left(3 M_{\odot}, 0.625 M_{\odot}\right)$, sufriendo un LTP. La edad cero corresponde al período pulsacional de 50 días. El tiempo está en unidades de $10^{3}$ años $(\phi=0.87)$. . . . .

24 VLTP: se muestra la evolución de un modelo post-AGB con masas $\left(M_{Z A M S}\right.$, $\left.M_{W D}\right)=\left(5 M_{\odot}, 0.836 M_{\odot}\right)$, sufriendo un VLTP. El tiempo está en unidades de $10^{3}$ años $(\phi=0.90) \ldots \ldots \ldots \ldots \ldots \ldots$

25 Trayectoria evolutiva sobre el diagrama $H-R$ de una estrella con masa $\left(M_{Z A M S}\right.$ , $\left.M_{W D}\right)=\left(2.7 M_{\odot}, 0.5885 M_{\odot}\right)$ que experimenta un VLTP en su fase temprana de enfriamiento después de que la quema de hidrógeno ha cesado. Se muestra la evolución desde la ZAMS, pasando por la fase de los pulsos térmicos, etapa de nebulosa planetaria, fase de 'born again', hasta la rama de enfriamiento de las enanas blancas. Los números sobre la trayectoria indican edades en miles de años donde se ha tomado el cero en el máximo de $L_{H e}$ del último pulso. Se indica el dominio de las estrellas PG1159, DO, DB y DQ, como así también el estado de pulsos térmicos, VLTP y born again. Después del episodio de born again el remanente post-AGB deficiente en hidrógeno experimenta una segunda incursión a la región de temperaturas bajas alcanzando posteriormente la rama de enfriamiento de las enanas blancas. Computada con nuestro código. . . . . . . . . . . . .

26 Pulsos térmicos para una estrella de $M_{Z A M S}=2.7 M_{\odot}$. Mostramos, a partir de nuestros resultados, la dependencia temporal de la luminosidad superficial ( $\left.L_{\text {sup }}\right)$, la luminosidad debida a la quema de hidrógeno por ciclo $C N O\left(L_{C N O}\right)$ y la debida a la quema de helio $\left(L_{H e}\right)$ en unidades solares. Después de experimentar 10 pulsos térmicos, el progenitor abandona la $A G B$, con una fase $\phi=0.89$, indicada en el gráfico con un punto negro. El gráfico de la derecha muestra el último pulso térmico que ocurre en la $A G B$ y el responsable del born again que se sucede en la fase post-AGB (pulso 11) cuando el remanente está en la rama de enfriamiento, ahí la luminosidad $L_{C N O}$ excede los $10^{8} L_{\odot}$. . . . . . . . . . . . . . . .

27 Para un modelo $\left(M_{Z A M S}, M_{W D}\right)=\left(2.7 M_{\odot}, 0.5885 M_{\odot}\right)$, se representa en el gráfico superior, la abundancia de ${ }^{4} \mathrm{He},{ }^{12} \mathrm{C} y{ }^{16} \mathrm{O}$ antes del primer pulso térmico. Ídem en el inferior, para el instante que realiza el primer codo en el azul en el diagrama $H-R$ (es el punto que en la fig. (25) está rotulado con $t=-12.7$ ). . . . . . . . 
28 Diagrama $H-R$ de los estados evolutivos de un pulso térmico muy tardío para una estrella con masa $\left(M_{Z A M S}, M_{W D}\right)=\left(2.7 M_{\odot}, 0.5885 M_{\odot}\right)$. El último pulso térmico es el responsable del episodio born again. Notar que el remanente experimenta una segunda incursión (doble loop) hacia la región de las gigantes rojas antes de evolucionar hacia el estado de enana blanca. El gráfico que se incluye muestra la dependencia temporal de la quema de hidrógeno por el ciclo CNO y por el helio (linea llena y punteada, respectivamente). Las letras seleccionadas indican: $A$ y $C$ corresponden al pico de la quema de helio $\left(L_{H e}\right)$ y al pico de la quema de hidrógeno por el ciclo CNO, respectivamente. En el punto B los protones comienzan a ser tragados y quemados por la zona convectiva del flash de helio. Entre los puntos E y $F$, la quema de hidrógeno se vuelve casi extinta, y el hidrógeno remanente alcanza el valor $1.3 \times 10^{-8} M_{\odot} \ldots \ldots \ldots \ldots \ldots \ldots \ldots$

29 Trayectoria completa de una estrella con $\left(M_{Z A M S}, M_{W D}\right)=\left(2.7 M_{\odot}, 0.5885 M_{\odot}\right)$. Se indican con círculos negros los distintos tiempos los cuales se corresponden a las figs. (31) a (39). Los círculos blancos representan instantes en determinados puntos característicos de la evolución. El cero se ha tomado en el máximo de la $L_{H e}$ (en el mismo lugar que en la fig. (25)). Obtenida de nuestros resultados. . . .

30 Para una estrella con masa $\left(M_{Z A M S}, M_{W D}\right)=\left(2.7 M_{\odot}, 0.5885 M_{\odot}\right)$ y para el último pulso encargado de generar el "born again", se grafican las luminosidades integradas $L_{\mathrm{He}}$ y $L_{C N O}$. Los círculos grises y blancos representan los tiempos dados en la fig. (29). Los tiempos que se indican sobre los círculos grises son los mismos que para los círculos blancos con igual abscisa. En el gráfico del recuadro inferior se indica que parte del pulso se muestra en forma ampliada. . . . . . . . . . . . . .

31 Interiores de los modelos estelares donde se indican las abundancias de ${ }^{1} \mathrm{H},{ }^{4} \mathrm{He}$, ${ }^{12} C,{ }^{13} C,{ }^{14} N y{ }^{16} \mathrm{O}$ como así también las regiones convectivas y el overshooting. Se grafica $\log q\left(\equiv \log \left(1-\frac{m_{r}}{M_{*}}\right)\right)$ vs. $\log X_{i}$, con $X_{i}$ : abundancia fraccional. El modelo superior corresponde a $t \simeq-42.8$ años y el inferior a $t \simeq-32.8$ años. . . . . . .

32 Idem fig. (31). El gráfico superior e inferior corresponde para $t \simeq-30.3$ y -1.88 años respectivamente. Observar el avance de la región convectiva. . . . . . . . .

33 Idem fig. (31). El gráfico superior e inferior corresponde a $t \simeq-0.47$ y 0.53 años respectivamente. La región convectiva avanza hacia afuera transformando los perfiles internos de abundancias. . . . . . . . . . . . . . . . . . .

34 Idem fig. (31). El gráfico superior e inferior corresponde para $t \simeq 0.72$ y 0.74 años respectivamente $(\Delta t \simeq 7.3$ días). El tiempo 0.74 es el del máximo de la luminosidad $L_{C N O} \ldots \ldots \ldots \ldots \ldots \ldots \ldots$

35 Idem fig. (31). El gráfico superior e inferior corresponde para $t \simeq 0.76$ y 0.78 años respectivamente $(\Delta t \simeq 7.3$ días $) . \ldots \ldots \ldots \ldots$ 
36 Idem fig. (31). El gráfico superior e inferior corresponde para $t \simeq 0.80$ y 0.82 años respectivamente $(\Delta t \simeq 7.3$ días). La región convectiva alcanzó la superficie, la estrella está en el vértice inferior y avanzando hacia el born again. El hidrógeno superficial comienza a disminuir para el modelo del gráfico inferior. . . . . . . . .

37 Idem fig. (31). El gráfico superior e inferior corresponde para $t \simeq 0.84$ y 0.98 años respectivamente $\left(\Delta t \simeq 51.1\right.$ días). El hidrógeno superficial es $X_{H} \simeq 0.018$. . . . .

38 Idem fig. (31). El gráfico superior e inferior corresponde para $t \simeq 1.15$ y 1.33 años respectivamente $\left(\Delta t \simeq 65.7\right.$ días). El hidrógeno superficial a $t \simeq 14.07$ es $X_{H} \simeq$

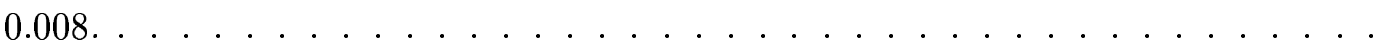

39 Idem fig. (31). El gráfico superior e inferior corresponde para $t \simeq 116.3$ y 982.6 años respectivamente. En el grafico superior es $X_{H} \simeq 3 \times 10^{-7}$ y en el inferior el hidrógeno superficial ha sido agotado casi por completo. . . . . . . . . . . .

40 Diagrama $H-R$ de la secuencia post-AGB para una estrella de $0.604 M_{\odot}$ de Herwig et al. (1999a;línea llena) con el VLTP y la subsiguiente evolución born again recalculada con $f_{v}=3$ (línea punteada, rotulada con " $A:$ :) y $f_{v}=30$ (línea de segmentos, rotulada como "B:"). El tiempo dado para $\log T_{\text {eff }}=3.9$ corresponde para el primer espectro presentado en Asplund et al. (1999) con fecha del 20 de abril de 1996. Todos los tiempos indicados están en años y dan el tiempo evolutivo desde el momento del pico de la gran luminosidad de la quema de hidrógeno $L_{H}$. Las rectas de trazos cortos representan tres magnitudes diferentes debajo de las cuales ha sido observada V4334 Sgr en diferentes momentos. En esta figura, se ha asumido que $d=4$ kpc y la luminosidad considerada es la de la trayectoria evolutiva de la estrella de $0.604 M_{\odot}$. Hay dos rectas segmentadas en donde se indican como límite superior (upper limit) debido a que V4334 Sgr ha sido vista por debajo o cerca del límite de detección. (Herwig, 2001) . . . . . . . . . . . . . .

41 Figura superior: mostramos la liberación de energía nuclear por quema de hidrógeno y helio, y el coeficiente de difusión (linea llena y segmentada, respectivamente, escala izquierda), y perfil de hidrógeno (línea punteada, escala derecha) para el máximo de luminosidad por quema de hidrógeno durante el último pulso térmico -punto $C$ de la fig. (28). Observar los picos de liberación de energía por quema de hidrógeno y helio debajo de la superficie estelar hacia 0.008 y $0.03 M_{\odot}$, respectivamente. Notar la presencia de las dos zonas convectivas (caraterizadas por grandes valores de D) las cuales están separadas por una región de inestabilidad salt finger. El parámetro de $O V$ es $f=0.015$. Figura inferior: al igual que en la superior, pero para un parámetro de $O V$ de $f=0.030$. Las 2 zonas convectivas están conectadas por una zona de $O V$, con la consecuencia de que algunos protones son ingeridos por la zona convectiva del flash de helio. . . . . . . . . . . . . . . . . . . 
42 Temperatura efectiva vs. tiempo para los estados evolutivos desde el último pulso térmico de helio y subsiguientes. Las líneas llenas y segmentadas corresponden a valores del parámetro de $O V f$ de 0.015 y 0.030, respectivamente. Para mayor claridad los primeros 9 años de la evolución son expandidos en el gráfico de la izquierda. En cada secuencia el cero en el tiempo corresponde al pico del pulso térmico de helio. Notar que en el caso de mayor eficiencia de $O V(f=0.030)$, la evolución born again es considerablemente mayor a aquella para el caso de $f=$ 0.015. Gráfico obtenido a partir de nuestros resultados. . . . . . . . . . .

43 Parte de la trayectoria evolutiva en el diagrama $H R$ de una estrella con una masa $\left(M_{Z A M S}, M_{W D}\right)=\left(2.7 M_{\odot}, 0.5885 M_{\odot}\right)$, en la etapa de estrella pre-enana blanca PG1159. Indicamos con círculos negros los cuatro tiempos correspondientes a la fig. (44). Los círculos grises indican los extremos de la banda de las PG1159. . . .

44 Figura superior: abundancias químicas internas de cuatro instantes evolutivos distintos para un modelo con una masa de $\left(M_{Z A M S}, M_{W D}\right)=\left(2.7 M_{\odot}, 0.5885 M_{\odot}\right)$, en el régimen de pre-enana blanca PG1159. Se representan las abundancias de ${ }^{4} \mathrm{He},{ }^{12} \mathrm{C},{ }^{13} \mathrm{C},{ }^{14} \mathrm{~N}$ y ${ }^{16} \mathrm{O}$ en función de la fracción de masa $q$ para el remanente de $0.5885 M_{\odot}$. Notar que en la figura para $t=669.9325 \times 10^{6}$ años aparece un aumento para el oxígeno como resultado de la captura de partículas a por el ${ }^{13} \mathrm{C}$. Figura inferior: idem fig. superior pero con las abundancias dadas en escala lo-

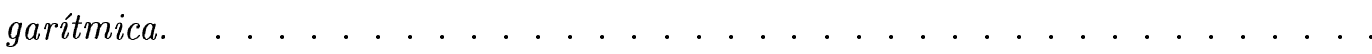

45 Diagrama de Hertzsprung-Russell esquemático ilustrando la localización de varias clases de estrellas enanas blancas. La banda gris representa la curva de enfriamiento de las enanas blancas. Sobre la misma se indica los distintos tipos espectrales de enanas blancas, se muestra también la brecha en donde están ausentes las DB y las pre-enanas blanca PG1159. . . . . . . . . . . . . . . .

46 Diagrama de Hertzsprung-Russell esquemático ilustrando la localización de varias clases de estrellas pulsantes. La línea de trazos muestra la secuencia principal, las curvas continuas representan algunas trayectorias evolutivas para masas de 1, 2, 3, 4, 7, 12 y $20 M_{\odot}$, la línea de trazos y puntos es la rama horizontal y la curva punteada es el track de enfriamiento de las enanas blancas (Christensen-Dalsgaard,

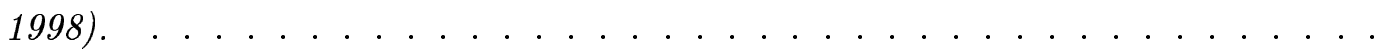

47 Diagrama $H-R$ de la rama de enfriamiento para el remanente de $0.5885 M_{\odot}$, los círculos negros representan los distintos modelos de las fig. (48), (49), (51) y (52). Las edades están en millones de años y las temperaturas efectivas en grados kelvin. Obtenido a partir de nuestros resultados. . . . . . . . . . . . . . 110 
48 Figura superior: abundancias químicas internas de cuatro modelos distintos para una estrella con $\left(M_{Z A M S}, M_{W D}\right)=\left(2.7 M_{\odot}, 0.5885 M_{\odot}\right)$, en las primeras etapas de la rama de enfriamiento de enana blanca. Figura inferior: idem figura superior pero con las abundancias dadas en escala logarítmica. . . . . . . . . . . . . 111

49 Figura superior: abundancias químicas internas de cuatro modelos distintos para una enana blanca DO con $\left(M_{Z A M S}, M_{W D}\right)=\left(2.7 M_{\odot}, 0.5885 M_{\odot}\right)$. Figura inferior: idem figura superior pero con las abundancias dadas en escala logarítmica. . . . . 112

50 Abundancia fraccional por masa para ${ }^{4} \mathrm{He} y{ }^{12} \mathrm{C}$ (línea llena segmentada, respectivamente) como una función de la fracción de masa exterior q para un modelo de $D B$ de $0.60 M_{\odot}$ para una temperatura $T_{\text {eff }}=27300 \mathrm{~K}$. Las líneas gruesas (delgadas) corresponden al caso en el cual la difusión térmica es considerada (despreciada). Claramente se ve que la inclusión de la difusión térmica conduce a mantos puros de helio más gruesos (Althaus and Córsico, 2004). . . . . . . . . . . . . .

51 Figura superior: abundancias químicas internas de cuatro modelos distintos para una enana blanca $D B$ con $\left(M_{Z A M S}, M_{W D}\right)=\left(2.7 M_{\odot}, 0.5885 M_{\odot}\right)$. Figura inferior: idem figura superior pero con las abundancias dadas en escala logarítmica. . . . . 114

52 Figura superior: abundancias químicas internas de dos modelos distintos para una enana blanca $D Q$ con $\left(M_{Z A M S}, M_{W D}\right)=\left(2.7 M_{\odot}, 0.5885 M_{\odot}\right)$. Figura inferior: idem figura superior pero con las abundancias dadas en escala logarítmica. . . . . 116 


\section{Índice de Tablas}

1 Reacciones nucleares consideradas en $L P C O D E \ldots \ldots \ldots \ldots$

2 Descripción de los puntos representativos de las figuras para una estrella de $M_{Z A M S}$ $=5 M_{\odot} \ldots \ldots \ldots \ldots \ldots \ldots \ldots \ldots \ldots \ldots \ldots \ldots \ldots \ldots \ldots \ldots \ldots$

$3 \quad$ Clasificación espectral de estrellas enanas blancas. . . . . . . . . . . 102

$4 \quad$ Núcleos más abundantes del sistema Solar . . . . . . . . . . . . . . . . . 147

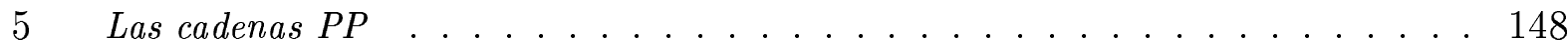

6 Los ciclos CNOF . . . . . . . . . . . . . . . . . . . . . 149

7 Ciclo $3-\alpha \ldots \ldots \ldots \ldots \ldots \ldots \ldots$

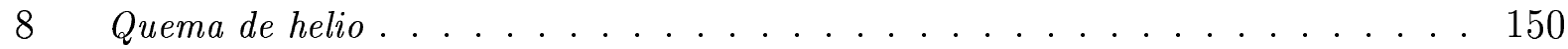

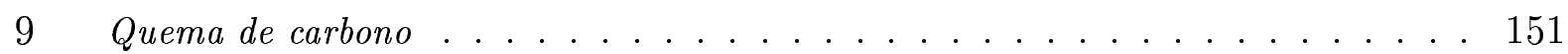

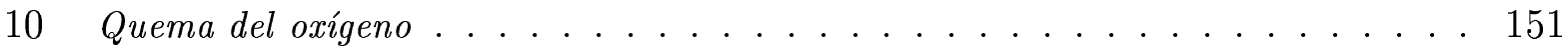

11 Fotodesintegración del neón . . . . . . . . . . . . . 152 


\section{Introducción}

En la actualidad, es un hecho bien establecido, a través de numerosos argumentos teóricos y observaciones, que el hidrógeno es el elemento más abundante del universo. En efecto, aproximadamente el 95\% de la masa del gas interestelar está compuesta de hidrógeno neutro (HI) y menos del 1\% de la masa total está constituida por elementos pesados y moléculas. La mayoría de las estrellas que pueblan el universo, aún en etapas avanzadas de su evolución, poseen hidrógeno primordial observable en sus envolturas. Sin embargo, desde hace algún tiempo los astrónomos han comenzado a detectar estrellas cuyos espectros revelan la ausencia de hidrógeno en sus atmósferas. Ejemplo de estrellas deficientes en hidrógeno son las variables supergigantes R Coronae Borealis (RCB), las PG1159 -las estrellas más calientes conocidas--, las estrellas centrales de nebulosas planetarias Wolf-Rayet (CSPNe: central Stars Planetary Nebulae), entre otras.

Desde las últimas décadas, la existencia de estrellas deficientes en hidrógeno ha sido objeto de intenso debate entre los astrofísicos. En parte, esto se debe a que los cálculos standard de evolución estelar predicen que la mayoría de las estrellas, aún en etapas muy avanzadas de su evolución, poseen una envoltura remanente rica en hidrógeno [por ej. (Vassiliadis and Wood, 1993a); (Blöcker, 1995a)]. Sin embargo, se han propuesto un número de posibles mecanismos que dan lugar a la formación de estrellas deficientes en hidrógeno. En efecto, existen varios escenarios que conducen a la formación de estrellas carentes de hidrógeno en sus envolturas. De acuerdo a Iben y colaboradores (Iben et al., 1996), los escenarios más ampliamente aceptados que conducen, por ejemplo, a la formación de las supergigantes RCB (con envolturas ricas en helio), involucran:

1) La ocurrencia de un flash termonuclear tardío (posterior a la quema de hidrógeno) en la capa rica en helio de una estrella central de nebulosa planetaria.

2) El colapso de un sistema binario en donde ambas componentes son enanas blancas, una de helio y otra de carbono-oxígeno, y cuya envoltura es común a ambas.

3) La fusión entre una estrella de neutrones y una estrella rica en helio.

Por otra parte, el escenario más ampliamente aceptado para explicar las WR CSPNe y sus descendientes más directos las PG1159 involucra la ocurrencia de un pulso térmico tardío en una estrella muy evolucionada, escenario conocido como born again. Dentro de esta categoría existen estrellas que presentan un espectro de helio casi puro y que estarían vinculadas a estrellas evolucionadas directamente de la rama horizontal extrema las cuales no experimentarían la etapa de los pulsos térmicos que ocurren durante la evolución a través 
de la rama asintótica gigante (AGB: Asymptotic Giant Branch) conocidas en la literatura como estrellas AGB-manqué.

Muchos de los canales evolutivos que predicen la formación de estrellas deficientes en hidrógeno no han sido explorados ampliamente y muchos de ellos están basados sobre consideraciones puramente especulativas, aún no puestas sobre bases teóricas firmes.

Antes de explicar como ocurre el escenario de born again, veamos qué sucede en su etapa previa. La fase AGB es la etapa más corta en la evolución de las estrellas de masa intermedia, y a pesar de su brevedad, es una fase evolutiva sumamente rica en lo que respecta a la nucleosíntesis. En efecto se cree que durante esta etapa se forma buena parte de los elementos más pesados que el hierro a través del proceso lento de captura de neutrones. En la fase AGB las estrellas están constituidas de un núcleo inerte de carbono-oxígeno rodeado de una capa delgada donde se quema helio. Encima de ésta, existe otra capa aún más delgada en la cual se quema el hidrógeno. Sobre ambas yace una envoltura convectiva. Durante la AGB se produce un dragado del material desde la base de la envoltura hacia la superficie, este evento es conocido como $3^{\text {er }}$ dredge up. A través del $3^{\text {er }}$ dredge-up la envoltura de la estrella es enriquecida con elementos pesados. Cabe mencionar que, en estrellas de masa intermedia existen dos etapas previas donde también se enriquece la superficie estelar con elementos procesados nuclearmente en el interior. Estas etapas son, el $1^{\text {er }}$ dredge up, que ocurre durante el primer ascenso de la estrella a la fase gigante, y el $2^{d o}$ dredge up, que tiene lugar en la rama asintótica gigante temprana (E-AGB: Early-AGB), antes de la AGB. El $2^{d o}$ dredge up sólo está presente en las estrellas más masivas de las estrellas de masa intermedia. Todos estos cambios de abundancias superficiales tienen lugar cuando la envoltura convectiva avanza hacia adentro en la estrella. Los cálculos teóricos detallados del $3^{e r}$ dredge up predicen abundancias químicas en la base de la envoltura en buen acuerdo con las abundancias superficiales observadas en las estrellas centrales de las nebulosas planetarias tipo Wolf-Rayet y en las PG1159.

El desarrollo de un pulso térmico tardío experimentado por un remanente post-AGB es, hoy en día, el mecanismo más altamente aceptado para explicar el origen de las enanas blancas deficientes en hidrógeno. En este sentido existen tres tipos de escenarios evolutivos que pueden llevar a la formación de estrellas deficientes en hidrógeno. Todos ellos invocan la necesidad de un pulso térmico final. Durante dicho pulso la envoltura de hidrógeno es completamente destruida por combustión nuclear o diluida por mezcla convectiva. Los escenarios evolutivos propuestos son [(Blöcker, 2001); (Herwig, 2001a)]: (i) un pulso térmico final (AFTP: AGB Final Thermal Pulse), que se produce muy tarde en la fase AGB y antes de salir de ésta; (ii) un pulso térmico tardío (LTP: Late Thermal Pulse), producido durante la evolución post-AGB cuando la quema de hidrógeno está todavía presente, sucediendo en su camino hacia altas temperaturas a luminosidad constante, en el diagrama $\mathrm{H}-\mathrm{R}$; y (iii) un 
pulso térmico muy tardío (VLTP: Very Late Thermal Pulse), que sucede cuando la estrella está sobre la rama de enfriamiento y la combustión del hidrógeno ha cesado. Todos estos escenarios conducen a estrellas post-AGB con baja abundancia superficial de hidrógeno: éste es diluido por el dredge up (en el caso de AFTP o LTP) o bien es completamente quemado (a través del VLTP).

En esta tesis nos concentraremos en el tercer escenario, pues aquí el hidrógeno remanente es consumido y por lo tanto es el canal más viable para explicar la existencia de las enanas blancas deficientes en hidrógeno. El remanente post-AGB está constituido por un núcleo de carbono-oxígeno, por encima del cual existe una capa delgada convectiva en la que el helio es quemado de manera inestable. Como consecuencia de los enormes flujos de energía que se generan, aparece una zona convectiva intermedia cuyo borde superior se propaga hacia la superficie de la estrella en menos de un año. Como resultado el hidrógeno externo es transportado hacia el interior donde finalmente es quemado mediante la reacción ${ }^{12} \mathrm{C}(p, \gamma){ }^{13} \mathrm{~N}$. El cálculo de estas etapas evolutivas es realmente muy complejo, porque la escala de tiempo en la cual ocurre la quema de protones, es comparable a la escala de tiempo característica de los procesos de mezcla convectiva. De modo que, la aproximación de mezcla instantánea, la cual es buena en otras etapas evolutivas, deja de ser válida. Un tratamiento de mezcla instantánea en esta etapa supondría que los protones se queman en la base de la envoltura, lo cual es incorrecto, ya que dada la rapidez con que ocurren las reacciones nucleares, los protones serán quemados en algún punto fuera de la envoltura, en su viaje hacia el interior de la estrella. Es aquí donde cobra importancia un tratamiento simultáneo de los cambios químicos producidos por reacciones nucleares y los debidos a los procesos de mezcla.

La energía liberada por quema de protones conduce eventualmente a un desdoblamiento de la región convectiva, en una zona superior que genera energía por la quema de hidrógeno, y otra inferior donde se quema helio. El flash producido es debido a que se están quemando protones a temperaturas excesivamente altas, del orden de $\approx 10^{8} \mathrm{~K}$, esto es a temperaturas típicas de quema de helio. Durante este pulso la mayor parte de hidrógeno remanente en la estrella es introducido dentro de la capa que quema helio y así es quemado casi por completo. La zona convectiva superior tiene una vida corta pues el hidrógeno de la envoltura es consumido rápidamente. Como resultado final la estrella se vuelve carente de hidrógeno, exponiendo en la superficie las abundancias de la intercapa. Luego del pulso la estrella se expande durante un período corto hacia las dimensiones de gigante roja. Esta fase de la estrella es la conocida como born again. Efectivamente la estrella sufre un rejuvenecimiento en su aspecto externo. Posteriomente, el remanente procede a quemar el helio durante una escala de tiempo larga, desandando aproximadamente (en el diagrama $\mathrm{H}-\mathrm{R}$ ) el camino seguido mientras quemó el hidrógeno en forma estable. A pesar de la brevedad de la duración de esta fase existen registros de objetos encontrados en esta etapa evolutiva. El caso más 
llamativo es el objeto Sakurai, V4334 Sagittarii, con un tiempo de escala born again del orden de 5 años, tiempo durante el cual el objeto ha aumentado su tamaño en un factor 1000. Esta clase de objetos ofrece una oportunidad importante para testear los cálculos de evolución estelar durante la etapa born again.

Después del escenario born again la estrella se desplaza en el diagrama $\mathrm{H}-\mathrm{R}$ por última vez hacia temperaturas elevadas y a luminosidad constante, hacia el dominio de las estrellas calientes PG1159. Gracias al desarrollo reciente de telescopios de alta resolución, las estrellas PG1159 están captando un interés creciente por parte de los investigadores. Muchas de ellas presentan pulsaciones, las cuales las convierten en candidatos ideales para el estudio de cuestiones que van más allá de la astrofísica estelar como por ejemplo la física de partículas. Las PG1159 constituyen un nexo evolutivo entre las estrellas supergigantes y las enanas blancas deficientes en hidrógeno. Las estrellas enanas blancas son el destino evolutivo final de las estrellas de masa baja e intermedia, esto es de la mayoría de las estrellas existentes en el universo. Puesto que aproximadamente un 20\% de estas estrellas carecen de hidrógeno, si las PG1159 son los antecesores directos de las enanas blancas deficientes en hidrógeno y además el escenario born again es el canal más viable para formar estas enanas blancas, entonces es claro que un porcentaje similar de las estrellas que se forman en el universo experimentarán un flash termonuclear tardío. Las PG1159 se encuentran en un rango de temperatura efectiva entre $75000 \mathrm{~K}$ y $180000 \mathrm{~K}$, y su prototipo es la estrella PG1159-035. La etapa PG1159 dura aproximadamente unos $10^{4}-10^{5}$ años. Después de este tiempo se apaga la capa que quema helio, la cual proveía la mayor parte de la luminosidad emergente de la estrella. El remanente ingresa entonces a lo que será su etapa evolutiva más lenta, la etapa de enana blanca.

El estudio de las estrellas enanas blancas es de gran importancia principalmente porque pueden ser consideradas como laboratorios naturales para explorar las propiedades de la materia bajo densidades y presiones extremas, no reproducibles en laboratorios terrestres. Muchas de estas estrellas presentan pulsaciones traducidas en variaciones multiperiódicas de su luminosidad. Las enanas blancas pulsantes constituyen una herramienta muy sensible para verificar la validez de los cálculos teóricos corrientes de estructura y evolución estelar. Los períodos de oscilación dependen sensiblemente de la estructura interna de las estrellas pulsantes, y en particular, de la forma precisa de los perfiles químicos internos. Tanto las observaciones como los análisis teóricos parecen confirmar una estructura en capa de las envolturas no-degeneradas de las enanas blancas. Obviamente, la validez de las pulsaciones estelares como una herramienta de diagnóstico de los interiores estelares depende justamente del grado de sofisticación y exactitud con que son construidos los modelos teóricos. Así, es crucial en este aspecto disponer de modelos de enanas blancas muy detallados, en particular en lo que respecta a la estructura química interna (perfiles de composición química). 
Como hemos mencionado la presente tesis está orientada hacia el estudio de los procesos de formación y evolución de las enanas blancas deficientes en hidrógeno. Para ello hemos realizado cálculos evolutivos detallados y completos, comenzando desde la secuencia principal, para modelos con masas de 2.5, 2.7 y $5 M_{\odot}$. Las simulaciones numéricas fueron extendidas de modo de cubrir las etapas evolutivas correspondientes a la rama de las gigantes rojas, la fase térmicamente pulsante AGB, el escenario born again, la etapa de PG1159 y terminando con la rama de enfriamiento de las enanas blancas.

Además de los objetivos delineados antes, parte de la labor desarrollada durante el trabajo de diploma consisitió en el cálculo de relaciones masa-radio para enanas blancas con núcleos de helio, carbono, oxígeno, silicio y hierro, para casos con envolturas de hidrógeno y helio, y envolturas sin hidrógeno. Todas estas relaciones masa-radio fueron computadas con el código de evolución estelar LPCODE. Las relaciones masa-radio son empleadas para confrontar predicciones teóricas sobre la composición interna de las enanas blancas con los datos observacionales. Muy recientemente han sido empleadas nuestras relaciones masaradio (Panei et al., 2000b) para el estudio de la distribución de masas de enanas blancas DA mediante datos del Sloan Digital Sky Survey (Madej et al., 2004). En dicho trabajo se determinaron masas, radios y luminosidades bolométricas de alrededor de 1300 enanas blancas. 


\section{Aspectos físicos y computacionales}

\subsection{Descripción general del código}

Para la realización de esta tesis se empleó nuestro código para evolución estelar LPCO$\mathrm{DE}^{1}$. Este programa ha sido desarrollado íntegramente en el Observatorio de La Plata.

El código está basado sobre el método de Kippenhahn, Weigert y Hofmeister (Kippenhahn et al., 1967) para calcular evolución estelar. Las integraciones de la envoltura son computadas para especificar las condiciones límites externas. Estas se realizan comenzando con valores desde la fotósfera hacia adentro de la estrella para un ajuste de la fracción de masa (cercana a la fotósfera). La variable independiente es $\xi=\ln \left(1-M_{r} / M_{*}\right.$ ) (llamada coordenada lagrangiana, también puede aparecer en esta tesis como $\xi=\ln (q)$, con $\left.q=1-M_{r} / M_{*}\right)$ y las variables dependientes son: radio $(r)$, presión $(P)$, luminosidad $(l)$ y temperatura $(T)$. Se consideran las siguientes variables, para el código:

$$
\begin{aligned}
\theta^{(n+1)} & =\theta^{(n)}+\ln \left(1+u_{\theta}\right) \\
p^{(n+1)} & =p^{(n)}+\ln \left(1+u_{p}\right) \\
x^{(n+1)} & =x^{(n)}+\ln \left(1+u_{x}\right) \\
l^{(n+1)} & =l^{(n)}+u_{l}
\end{aligned}
$$

siendo $u_{\theta}, u_{p}, u_{x} \mathrm{y} u_{l}$ las cantidades a iterar $u_{\theta}=\frac{\Delta T}{T^{(n)}}, u_{p}=\frac{\Delta P}{P^{(n)}}, u_{x}=\frac{\Delta r}{r^{(n)}}$ y $u_{l}=\Delta l$, donde los subíndices $n$ y $n+1$ indican el comienzo y el fin de un intervalo temporal -ver (Kippenhahn et al., 1967). Aquí, $\theta=\ln T, x=\ln r$ y $p=\ln P$. Así, el esquema de iteración de Henyey está aplicado a las diferencias en la luminosidad, presión, temperatura y radio entre el modelo previo y el calculado.

El código LPCODE emplea las opacidades radiativas OPAL (que tienen en cuenta composiciones ricas en carbono y oxígeno) para metalicidades arbitrarias dadas por Iglesias y Rogers (Iglesias and Rogers, 1996) y las de Alexander y Ferguson (Alexander and Ferguson, 1994) para regímenes de bajas temperaturas. Se emplean las opacidades para distintas metalicidades, durante la etapa de enfriamiento de la enana blanca, según el gradiente de metalicidad que se desarrolla en la envoltura de los modelos, como un resultado del asentamiento gravitacional. La ecuación de estado para el régimen de baja densidad comprende ionización parcial para composiciones de hidrógeno y helio, presión de radiación y la contribución de los iones. Para el régimen de altas densidades se consideran a los electrones parcialmente degenerados e interacciones coulombianas. Para el régimen de enana blanca se

\footnotetext{
${ }^{1}$ Este código está escrito en lenguaje FORTRAN 77 y posee más de 27000 líneas.
} 
ha incluido una versión actualizada de la ecuación de estado dada por Magni y Mazzitelli (Magni and Mazzitelli, 1979). En el código LPCODE la cristalización se considera cuando la constante de acoplamiento, $\Gamma \equiv Z^{2} e^{2} / \bar{r} k_{B} T$ alcanza el valor 180 .

Para la tasa de emisión de neutrinos: las opacidades conductivas empleadas y la emisión de neutrinos por Bremsstrahlung en la fase de cristalización son tomadas de los trabajos de Itoh y colaboradores -(Itoh et al., 1984a); (Itoh et al., 1984b); ver también fe de erratas (Itoh et al., 1987). Para describir la quema de hidrógeno (cadena protón-protón y ciclo CNO) y de helio, e ignición de carbono, se ha considerado una red de 34 reacciones termonucleares - ver tabla [(1), sec. (2.1)]- con 17 isótopos intervinientes en la reacción. Los considerados son: ${ }^{1} \mathrm{H},{ }^{2} \mathrm{H},{ }^{3} \mathrm{He},{ }^{4} \mathrm{He},{ }^{7} \mathrm{Li},{ }^{7} \mathrm{Be},{ }^{12} \mathrm{C},{ }^{13} \mathrm{C},{ }^{14} \mathrm{~N},{ }^{15} \mathrm{~N},{ }^{16} \mathrm{O},{ }^{17} \mathrm{O},{ }^{18} \mathrm{O},{ }^{19} \mathrm{~F},{ }^{20} \mathrm{Ne},{ }^{22} \mathrm{Ne}$ y ${ }^{24} \mathrm{Mg}$.

\begin{tabular}{|c|c|}
\hline$\overline{7} \mathrm{Be}+e^{-} \rightarrow{ }^{7} \mathrm{Li}+\nu$ & $p+p+e^{-} \rightarrow d+\nu$ \\
\hline$p+p \rightarrow d+e^{+}+\nu$ & $p+d \rightarrow{ }^{3} \mathrm{He}+\gamma$ \\
\hline$p+{ }^{3} \mathrm{He} \rightarrow{ }^{4} \mathrm{He}+\gamma$ & ${ }^{3} \mathrm{He}+{ }^{4} \mathrm{He} \rightarrow{ }^{7} \mathrm{Be}+\gamma$ \\
\hline$p+{ }^{7} \mathrm{Be} \rightarrow{ }^{4} \mathrm{He}+{ }^{4} \mathrm{He}$ & $p+{ }^{7} \mathrm{Li} \rightarrow{ }^{4} \mathrm{He}+{ }^{4} \mathrm{He}$ \\
\hline${ }^{4} \mathrm{He}+{ }^{4} \mathrm{He} \rightarrow p+p+{ }^{4} \mathrm{He}$ & $\begin{aligned} p+{ }^{12} \mathrm{C} & \rightarrow\left({ }^{13} \mathrm{~N}+\gamma\right. \\
/{ }^{13} \mathrm{~N} & \rightarrow){ }^{13} \mathrm{C}+e^{+}+\nu\end{aligned}$ \\
\hline$p+{ }^{13} \mathrm{C} \rightarrow{ }^{14} \mathrm{~N}+\gamma$ & $\begin{array}{l}p+{ }^{14} \mathrm{~N} \rightarrow\left({ }^{15} \mathrm{O}+\gamma\right. \\
\left.\quad /{ }^{15} \mathrm{O} \rightarrow\right){ }^{15} \mathrm{~N}+e^{+}+\nu\end{array}$ \\
\hline$p+{ }^{15} \mathrm{~N} \rightarrow{ }^{16} \mathrm{O}+\gamma$ & $p+{ }^{15} \mathrm{~N} \rightarrow{ }^{4} \mathrm{He}+{ }^{12} \mathrm{C}$ \\
\hline$p+{ }^{16} \mathrm{O} \rightarrow\left({ }^{17} \mathrm{~F}+\gamma\right.$ & $p+{ }^{17} \mathrm{O} \rightarrow\left({ }^{18} \mathrm{~F}+\gamma\right.$ \\
\hline $\begin{aligned} /{ }^{17} \mathrm{~F} & \rightarrow){ }^{17} \mathrm{O}+e^{+}+\nu \\
p+{ }^{17} \mathrm{O} & \rightarrow{ }^{4} \mathrm{He}+{ }^{14} \mathrm{~N}\end{aligned}$ & $\begin{array}{l}\left.\quad /{ }^{18} \mathrm{~F}+e^{-} \rightarrow\right){ }^{18} \mathrm{O}+\nu \\
p+{ }^{18} \mathrm{O} \rightarrow{ }^{4} \mathrm{He}+{ }^{15} \mathrm{~N}\end{array}$ \\
\hline$p+{ }^{18} \mathrm{O} \rightarrow{ }^{19} \mathrm{~F}+\gamma$ & $p+{ }^{19} \mathrm{~F} \rightarrow{ }^{20} \mathrm{Ne}+\gamma$ \\
\hline$p+{ }^{19} \mathrm{~F} \rightarrow{ }^{4} \mathrm{He}+{ }^{16} \mathrm{O}$ & $\begin{array}{l}{ }^{4} \mathrm{He}+{ }^{4} \mathrm{He}\left(\rightarrow{ }^{8} \mathrm{Be}\right. \\
\left.\quad /{ }^{8} \mathrm{Be}\right)+{ }^{4} \mathrm{He} \rightarrow{ }^{12} \mathrm{C}+\gamma\end{array}$ \\
\hline${ }^{4} \mathrm{He}+{ }^{12} \mathrm{C} \rightarrow{ }^{16} \mathrm{O}+\gamma$ & ${ }^{4} \mathrm{He}+{ }^{16} \mathrm{O} \rightarrow{ }^{20} \mathrm{Ne}+\gamma$ \\
\hline${ }^{4} \mathrm{He}+{ }^{14} \mathrm{~N} \rightarrow{ }^{18} \mathrm{O}$ & ${ }^{4} \mathrm{He}+{ }^{15} \mathrm{~N} \rightarrow{ }^{19} \mathrm{~F}+\gamma$ \\
\hline${ }^{4} \mathrm{He}+{ }^{20} \mathrm{Ne} \rightarrow{ }^{24} \mathrm{Mg}+\gamma$ & ${ }^{4} \mathrm{He}+{ }^{17} \mathrm{O} \rightarrow p+{ }^{20} \mathrm{Ne}$ \\
\hline${ }^{12} \mathrm{C}+{ }^{12} \mathrm{C} \rightarrow{ }^{24} \mathrm{Mg}+\gamma$ & ${ }^{12} \mathrm{C}+{ }^{12} \mathrm{C} \rightarrow{ }^{4} \mathrm{He}+{ }^{20} \mathrm{Ne}$ \\
\hline${ }^{4} \mathrm{He}+{ }^{13} \mathrm{C}+\gamma \rightarrow n+{ }^{16} \mathrm{O}$ & ${ }^{4} \mathrm{He}+{ }^{18} \mathrm{O} \rightarrow{ }^{22} \mathrm{Ne}+\gamma$ \\
\hline${ }^{4} \mathrm{He}+{ }^{22} \mathrm{Ne} \rightarrow n+{ }^{25} \mathrm{Mg}$ & ${ }^{4} \mathrm{He}+{ }^{22} \mathrm{Ne} \rightarrow{ }^{26} \mathrm{Mg}+\gamma$ \\
\hline
\end{tabular}

Las redes de reacciones nucleares son tomadas de Caughlan y Fowler (Caughlan and Fowler, 1988), excepto para las reacciones ${ }^{15} \mathrm{~N}(p, \gamma){ }^{16} \mathrm{O},{ }^{15} \mathrm{~N}(p, \alpha){ }^{12} \mathrm{C},{ }^{18} \mathrm{O}(p, \alpha){ }^{15} \mathrm{~N},{ }^{18} \mathrm{O}(p, \gamma){ }^{19} \mathrm{~F}$, 
${ }^{12} \mathrm{C}(\alpha, \gamma){ }^{16} \mathrm{O},{ }^{16} \mathrm{O}(\alpha, \gamma){ }^{20} \mathrm{Ne},{ }^{13} \mathrm{C}(\alpha, n){ }^{16} \mathrm{O},{ }^{18} \mathrm{O}(\alpha, \gamma){ }^{22} \mathrm{Ne},{ }^{22} \mathrm{Ne}(\alpha, n){ }^{25} \mathrm{Mg}$ y ${ }^{22} \mathrm{Ne}(\alpha, \gamma){ }^{26} \mathrm{Mg}$, las cuales se han obtenido a partir del trabajo de Angulo (Angulo, 1999). En particular la tasa de reacción ${ }^{12} \mathrm{C}(\alpha, \gamma){ }^{16} \mathrm{O}$ dada por Angulo, es de alrededor de dos veces más grande que la de Caughlan y Fowler. En la tabla [(1), sec. (2.1)] se han tenido en cuenta reacciones que involucran $n,{ }^{25} \mathrm{Mg}$ y ${ }^{26} \mathrm{Mg}$, debido a su importancia en la evolución estelar.

Los pasos temporales evolutivos durante la fase de los pulsos térmicos van desde unos pocos días durante los flashes del helio y las subsecuentes fases donde ocurre el tercer dredge up, hasta algunos años durante la fase estacionaria de la quema de hidrógeno en los interpulsos.

\subsection{Evolución química}

Un aspecto importante del presente estudio es el modelado de la distribución de las abundancias químicas a través de todas las diferentes fases evolutivas. Para este fin consideramos un esquema dependiente del tiempo para el tratamiento simultáneo de los cambios químicos causado por la quema nuclear y la mezcla convectiva, semiconvectiva, inestabilidad tipo Rayleigh-Taylor (salt finger) y overshooting (OV) -ver sec. (13.4). Tal acoplamiento entre la evolución nuclear y la mezcla dependiente del tiempo es mucho más realista que la mezcla instantánea, aproximación que es usualmente asumida en el modelado estelar. En particular esta clase de tratamiento ha sido usado por Mazzitelli y colaboradores (Mazzitelli et al., 1999) para estudiar la producción de litio por el hot bottom burning en las estrellas de la AGB (Ventura et al., 1999).

Presentamos a continuación algunos detalles del método numérico para los cambios de abundancias incluidos en LPCODE. Los cambios de abundancias para todos los elementos químicos están descriptos por el conjunto de ecuaciones:

$$
\left(\frac{d \vec{Y}}{d t}\right)=\left(\frac{\partial \vec{Y}}{\partial t}\right)_{\mathrm{nuc}}+\frac{\partial}{\partial M_{r}}\left[\left(4 \pi r^{2} \rho\right)^{2} D \frac{\partial \vec{Y}}{\partial M_{r}}\right]
$$

donde $\vec{Y}$ es un vector que contiene la fracción numérica de todas las especies nucleares consideradas. Aquí la mezcla debida a la convección, semiconvección, salt finger y overshooting es tratada como un proceso de difusión el cual está descripto en el segundo término de la ec. (2) en términos de la coordenada masa $M_{r}$. La eficiencia del proceso de mezcla está descripto por coeficientes de difusión apropiados $D$ (ver más adelante). El primer término de la ec. (2), da los cambios de abundancias debido a las reacciones termonucleares, los cuales están acoplados con el proceso de mezcla. Este término es desarrollado como una función de abundancias locales. Empleando el esquema de Arnett y Truran (Arnett and Truran, 1969), después de la linealización podemos escribir: 


$$
\left(\frac{\vec{Y}_{j}^{n+1}-\vec{Y}_{j}^{n}}{\Delta t}\right)_{\mathrm{nuc}}=-\Gamma_{j} \vec{Y}^{n+1}+\vec{\Lambda}_{j},
$$

donde $\Delta t$ es el intervalo de tiempo $\Delta t=t^{n+1}-t^{n}$ y los supraíndices $n$ y $n+1$ indican el comienzo y el final del intervalo. El subíndice $j$, indica el $j^{\text {ésimo }}$ punto del grillado en que se divide el interior estelar. $\Gamma_{j}$ es una matriz de $N \times N$ (donde $N$ es le número de especies nucleares consideradas) y $\vec{\Lambda}_{j}$ es un vector de dimensión $N$, ambos con elementos que involucran las abundancias $\left(\vec{Y}_{j}^{n}\right)$ y tasas de reacciones nucleares para $t^{n}$. Usando el esquema de diferencias finitas de tres puntos, el segundo término de la ec. (2) puede ser aproximado por

$$
\left\{\frac{\partial}{\partial M_{r}}\left[\left(4 \pi r^{2} \rho\right)^{2} D \frac{\partial \vec{Y}}{\partial M_{r}}\right]\right\}_{j}^{n+1}=\mathcal{A} \vec{Y}_{j-1}^{n+1}+\mathcal{B} \vec{Y}_{j}^{n+1}+\mathcal{C} \vec{Y}_{j+1}^{n+1} .
$$

donde $\mathcal{A}, \mathcal{B}$ y $\mathcal{C}$ son matrices diagonales con una dimensión de $N \times N$. Específicamente, los elementos no nulos de tales matrices están dados por:

$$
\begin{gathered}
a_{i i}=\frac{(4 \pi)^{2}\left(\rho^{2} r^{4} D\right)_{j-1 / 2}}{\left(m_{j-1 / 2}-m_{j+1 / 2}\right)\left(m_{j-1}-m_{j}\right)} \\
c_{i i}=\frac{(4 \pi)^{2}\left(\rho^{2} r^{4} D\right)_{j+1 / 2}}{\left(m_{j-1 / 2}-m_{j+1 / 2}\right)\left(m_{j}-m_{j+1}\right)} \\
b_{i i}=-\left(a_{i i}+c_{i i}\right),
\end{gathered}
$$

donde los subíndices $j+1 / 2(j-1 / 2)$ indica el promedio para la masa de la capa ubicada entre el punto del grillado $j$ y $j+1(j-1)$. Para los límites del intervalo de integración de masa, no tenemos flujo de masa, no hay sumidero $\left(\partial \vec{Y} / \partial M_{r}=0\right)$, así:

$$
\frac{\partial}{\partial M_{r}}\left[\left(4 \pi r^{2} \rho\right)^{2} D \frac{\partial \vec{Y}}{\partial M_{r}}\right]_{\text {borde }}=\left(4 \pi r^{2} \rho\right)^{2} D \frac{\partial^{2} \vec{Y}}{\partial M_{r}^{2}} .
$$

Una expansión de Taylor de segundo orden en las abundancias conducen a

$$
c_{i i}=2 \frac{(4 \pi)^{2}\left(\rho^{2} r^{4} D\right)_{1 / 2}}{\left(m_{1}-m_{2}\right)^{2}} ; \quad b_{i i}=-c_{i i} ; \quad a_{i i}=0
$$


para la condición de borde superior, y a

$$
a_{i i}=2 \frac{(4 \pi)^{2}\left(\rho^{2} r^{4} D\right)_{J-1 / 2}}{\left(m_{J-1}-m_{J}\right)^{2}} ; \quad b_{i i}=-a_{i i} ; \quad c_{i i}=0
$$

para la condición de borde inferior (para el jésimo punto del grillado).

Las ecs. (2), (3) y (4) conducen al siguiente sistema de ecuaciones lineales para ser resueltas simultáneamente para las nuevas abundancias químicas $\vec{Y}^{n+1}$ para el tiempo $t^{n+1}$ :

$$
\mathcal{F}_{j} \vec{Y}_{j}^{n+1}-\mathcal{A}_{j} \vec{Y}_{j-1}^{n+1}-\mathcal{C}_{j} \vec{Y}_{j+1}^{n+1}=\overrightarrow{\mathcal{M}}_{j}
$$

donde $\mathcal{F}_{j}=\mathcal{T}^{-1}+\Gamma_{j}-\mathcal{B}_{j}$ y $\overrightarrow{\mathcal{M}}_{j}=\vec{\Lambda}_{j}+\vec{Y}_{j}^{n} / \Delta t . \mathcal{T}^{-1}$ es una matriz diagonal de $N \times N$ con elementos ${ }^{2} 1 / \Delta t$. Afortunadamente, la ec. (11) tiene una estructura especial (tridiagonal) la cual nos permite minimizar el almacenamiento de la matriz de los coeficientes. Esquemáticamente, tenemos $\left(\mathcal{A}_{j}, \mathcal{F}_{j}\right.$ y $\mathcal{C}_{j}$ son matrices y $\vec{Y}_{j}^{n+1}$ y $\overrightarrow{\mathcal{M}}_{j}$ son vectores $)$

$$
\left(\begin{array}{ccccccccc}
\mathcal{F}_{1} & \mathcal{C}_{2} & & & & & & & \\
\mathcal{A}_{1} & \mathcal{F}_{2} & \mathcal{C}_{3} & & & & & & \\
& \mathcal{A}_{2} & \mathcal{F}_{3} & \mathcal{C}_{4} & & & & & \\
& & \ddots & \ddots & \ddots & & & & \\
& & & \ddots & \ddots & \ddots & & & \\
& & & \ddots & \ddots & \ddots & & \\
& & & & \mathcal{A}_{J-3} & \mathcal{F}_{J-2} & \mathcal{C}_{J-1} & \\
& & & & & \mathcal{A}_{J-2} & \mathcal{F}_{J-1} & \mathcal{C}_{J} \\
& & & & & & \mathcal{A}_{J-1} & \mathcal{F}_{J}
\end{array}\right) \bullet\left(\begin{array}{c}
\vec{Y}_{1}^{n+1} \\
\vec{Y}_{2}^{n+1} \\
\vec{Y}_{3}^{n+1} \\
\vdots \\
\vdots \\
\vdots \\
\vec{Y}_{J-2}^{n+1} \\
\vec{Y}_{J-1}^{n+1} \\
\vec{Y}_{J}^{n+1}
\end{array}\right)=\left(\begin{array}{c}
\overrightarrow{\mathcal{M}}_{1} \\
\overrightarrow{\mathcal{M}}_{2} \\
\overrightarrow{\mathcal{M}}_{3} \\
\vdots \\
\vdots \\
\vdots \\
\overrightarrow{\mathcal{M}}_{J-2} \\
\overrightarrow{\mathcal{M}}_{J-1} \\
\overrightarrow{\mathcal{M}}_{J}
\end{array}\right)
$$

Para resolver este conjunto de ecuaciones, seguimos el método considerado por Iben y MacDonald (Iben and MacDonald, 1985). La ec. (2) es aplicada a las zonas radiativas $(D=0)$ y para las regiones convectivas, semiconvectivas, con overshooting y salt finger dando el coeficiente de difusión $D$, el cual es especificado para cada proceso de acuerdo al tratamiento de convección adoptado. Los diecisiete $(N=17)$ isótopos utilizados son los mencionados en la sección (2.1). En LPCODE, los cambios de abundancias son recalculados después de la convergencia de cada modelo estelar (y no durante las iteraciones). Para una mejor precisión en la integración durante el cómputo de la evolución nuclear, cada paso de tiempo evolutivo es dividido en 5 pasos de tiempo químico.

\footnotetext{
${ }^{2}$ Notar que la ec. (11) acopla la evolución nuclear al actual cambio de composición debido al proceso de mezcla.
} 
La evolución de la distribución de abundancia química causada por la difusión de los elementos químicos durante toda la evolución de la enana blanca constituye un punto importante en los cálculos computacionales. En nuestro tratamiento de la difusión dependiente del tiempo hemos considerado el asentamiento gravitacional y la difusión química y térmica para las siguientes especies nucleares: ${ }^{1} \mathrm{H},{ }^{3} \mathrm{He},{ }^{4} \mathrm{He},{ }^{12} \mathrm{C},{ }^{14} \mathrm{~N}$ y ${ }^{16} \mathrm{O}$. La evolución química resultante de la difusión de los elementos está descripta, para un dado isótopo $i$, por la ecuación de continuidad dada por

$$
\left(\frac{\partial Y_{i}}{\partial t}\right)_{\text {dif }}=-\frac{\partial}{\partial M_{r}}\left(4 \pi r^{2} \rho Y_{i} w_{i}\right)
$$

donde $w_{i}$ es la velocidad de difusión. Hemos adoptado el tratamiento dado por Burgers (Burgers, 1969) para gases compuestos. Nos ocuparemos de la evolución química que ocurre muy adentro en la estrella, así la levitación radiativa (la cual modificaría la composición superficial de la enanas blancas calientes) ha sido despreciada. Las velocidades de difusión, en término del gradiente de densidades de los iones, pueden escribirse de la forma

$$
w_{i}=w_{i}^{\mathrm{gt}}-\sum_{\operatorname{ions}(j)} \sigma_{i j} \frac{d \ln n_{j}}{d r}
$$

donde $w_{i}^{\text {gt }}$ representa la componente de velocidad debida al asentamiento gravitacional y a la difusión térmica. Por mayores detalles ver Althaus y Benvenuto (Althaus and Benvenuto, 2000); también Gautschy y Althaus (Gautschy and Althaus, 2002) para una aplicación a las enanas blancas DB. Los cambios de abundancia resultantes de la difusión de los elementos están completamente acoplados a las reacciones nucleares. Para este fin, la matriz de los coeficientes en la ec. (12) ha sido modificada en forma apropiada.

\subsection{Overshooting (OV)}

Se tiene en cuenta la mezcla por OV dependiente del tiempo durante todos los estados de la evolución pre-enana blanca. El esquema para los cambios de abundancia descriptos anteriormente nos permite un tratamiento autoconsistente del OV difusivo en presencia de la quema nuclear. En particular hemos considerado un decaimiento exponencial para el OV difusivo por arriba y por debajo de cualquier región convectiva, incluyendo el núcleo convectivo (en las fases de secuencia principal y quema central de helio), la envoltura convectiva externa y durante la corta vida de la zona convectiva en el flash de helio la cual desarrolla durante los pulsos térmicos. Se ha seguido el formalismo de Herwig (Herwig, 2000) basado en las simulaciones hidrodinámicas de Freytag y colaboradores (Freytag et al., 1996), lo 
cual muestra que las velocidades turbulentas decaen exponencialmente fuera de los límites convectivos. La expresión para el coeficiente de difusión en las regiones de OV es

$$
D_{\mathrm{os}}=D_{0} \exp \left(\frac{-2 z}{H_{\mathrm{v}}}\right),
$$

donde $D_{0}$ es el coeficiente de difusión en el límite de la zona convectiva, $z$ es la distancia radial desde el borde de la zona convectiva, $H_{\mathrm{v}}=f H_{\mathrm{P}}$, donde el parámetro libre $f$ es una medida de la extensión de la región del $\mathrm{OV}$, y $H_{\mathrm{P}}$ es la altura de escala de la presión para el límite convectivo. Hemos asumido que $f=0.015$, lo cual tiene en cuenta el ancho observado de la secuencia principal así como las abundancias deficientes de hidrógeno entre las capas de los remanentes post-AGB -ver (Herwig et al., 1997); (Herwig et al., 1999a); (Herwig, 2000) para detalles; ver también (Mazzitelli et al., 1999).

\subsection{Tratamiento de la convección}

El programa LPCODE tiene en cuenta una teoría ampliada de la mixing length (longitud de mezcla) para la convección, para fluidos con gradientes de composición, desarrollado por Grossman y colaboradores (Grossman et al., 1993) en su aproximación local como la dada por Grossman y Taam (Grossman and Taam, 1996). Estos autores han desarrollado una teoría de la mixing length no-lineal de convección doble difusiva que se aplica en los regímenes inestables convectivos, semiconvectivos e inestabilidad Rayleigh-Taylor (salt finger). De acuerdo con este tratamiento, el coeficiente de difusión $D$ en la ec. (2) caracterizando tales regímenes de mezcla, está dado por:

$$
D=\ell \sigma
$$

donde $\ell=\alpha H_{P}$ es la longitud de mezcla y $\sigma$ la velocidad turbulenta. El valor de $\sigma$ está determinado por la resolución simultánea de las ecuaciones para la velocidad turbulenta y la conservación de flujo -ec. (9) y (17) de (Grossman and Taam, 1996). Aquí el parámetro $\alpha$ es tomado como 1.5 .

\subsection{Tasa de pérdida de masa}

El tratamiento para la pérdida de masa es aquel empleado por Blöcker (Blöcker, 1995b). En particular, durante la evolución en la AGB, la tasa de pérdida de masa está dada por

$$
\dot{M}=4.83 \times 10^{-9} M_{\mathrm{ZAMS}}^{-2.1} L^{2.7} \dot{M}_{\mathrm{R}}\left[M_{\odot} / y r\right]
$$


donde $\dot{M}_{\mathrm{R}}$ es la tasa de pérdida de masa de Reimer dada por $\dot{M}_{\mathrm{R}}=4 \times 10^{-13} \eta_{\mathrm{R}} L R / M$ con $L, R$ y $M$ luminosidad, radio y masa de la estrella en unidades solares. Esta formulación está basada sobre cómputos dinámicos para atmósferas de estrellas tipo Mira, teniendo en cuenta un fuerte aumento de la tasa de pérdida de masa y modulaciones por los pulsos térmicos esperados en el curso de la evolución AGB. Tomamos el valor del parámetro libre $\eta_{\mathrm{R}}$ en forma algo arbitraria (igual a 1) en lugar de obtener un razonable número de pulsos térmicos sobre la AGB. En nuestro cómputo, los episodios de pérdida de masa que toman lugar durante los estados de quema nuclear de helio y de la rama gigante roja (RGB: Red Giant Branch) son considerados de acuerdo a la formulación usual de Reimer con $\eta_{\mathrm{R}}=1$. 


\section{Parte I}

\section{Relación Masa-Radio}




\section{Relaciones masa-radio}

En esta sección mostraremos resultados sobre relaciones masa-radio para enanas blancas con composiciones internas de helio, carbono, oxígeno, silicio y hierro, con envolturas compuestas de hidrógeno y helio o sin hidrógeno.

\subsection{Masa límite de Chandrasekhar}

Chandrasekhar (Chandrasekhar, 1939) demostró, que partiendo del siguiente sistema de ecuaciones, constituido por la ecuación de equilibrio hidrostático, distribución de masa y la ecuación de estado para un gas de electrones degenerados a temperatura cero

$$
\frac{\partial P}{\partial r}=-\frac{G m}{r^{2}} \varrho \quad, \quad \frac{\partial m}{\partial r}=4 \pi r^{2} \varrho \quad, \quad P=P\left(\varrho, \mu_{e}\right)
$$

se puede reformular el problema a través de una ecuación diferencial de segundo orden (la que gobierna la estructura mecánica de una enana blanca) para una función sin dimensiones, con dos parámetros: $\mu_{e}$, el peso molecular por electrón y $\varrho_{c}$, la densidad central. Esta función debe ser determinada numéricamente. Sin embargo, es fácil ver, como puede obtenerse la solución a partir del sistema de ecs. (18), para un valor dado de $\mu_{e}$, es decir una composición química determinada, y un valor elegido de $\varrho_{c}$-ver Apéndice A, sec. (13.7.3). La integración del sistema de ecs. (18) se realiza desde el centro $(r=0)$ hasta que se alcanza el valor $P(r)=0$ (en la superficie). Esto determina el radio $R$ (radio de la configuración) y la masa $M=M(R)$ del modelo (masa total). Para un valor dado de $\mu_{e}$ se obtiene una única familia de modelos, definiendo implícitamente una relación entre la masa y el radio, la famosa "relación masa-radio" obtenida por Chandrasekhar (Chandrasekhar, 1935). En el límite $\varrho_{c} \rightarrow \infty, R$ se vuelve cero, mientras que la masa se aproxima al valor límite

$$
M_{C h}=1.459 \times\left(\frac{2}{\mu_{e}}\right)^{2} M_{\odot}
$$

la "masa límite de Chandrasekhar" (ver Apéndice A, sec. 13.7.3 caso ultrarelativista).

\subsection{Refinamientos a la teoría de Chandrasekhar: modelos de Ha- mada y Salpeter}

Aunque en el gas ideal, los electrones no interactúan, éste da idea de la contribución dominante a la ecuación de estado para altas densidades. Pero un plasma real consiste en electrones junto con iones pesados y positivamente cargados de una o más especies. 
Las propiedades termodinámicas de la materia están completamente determinadas si se conoce la energía libre de Helmholtz $F(T, V, N)$ (T: temperatura, V: volumen, N: número de partículas) del sistema (Fermi, 1936). Entonces la presión, puede ser obtenida mediante:

$$
P=-\left(\frac{\partial F}{\partial V}\right)_{T, N}
$$

Puesto que $F=E-T \cdot S$ (E: energía interna, S: entropía), a temperatura cero sólo es necesario conocer $E(V, N)$.

La energía del plasma puede escribirse como la suma de la energía cinética de los electrones más los términos de corrección, de los cuales la interacción coulombiana es el mayor (Salpeter, 1961).

$$
E=E_{e}+E_{c}+E_{e x}+E_{T F}+E_{c o r r}
$$

$E_{e}$ : energía cinética de los electrones, está relacionada a la energía de Fermi.

$E_{c}$ : energía de interacción coulombiana.

$E_{e x}$ : energía de intercambio (exchange), esta contribución proviene del hecho de que los electrones son partículas indistinguibles, en la mecánica cuántica.

$E_{T F}$ : corrección de Thomas-Fermi, la contibución de Thomas-Fermi tiene en cuenta el efecto producido por el hecho de que la distribución de electrones está polarizada por los iones cargados positivamente.

$E_{c o r r}$ : energía de correlación, proviene de si la energía de interacción entre electrones está expandida en potencias de $r_{i}$ (la separación típica entre iones).

Con la energía total determinada por la ec. (21), la presión para la ecuación de estado se obtiene como:

$$
P=P_{e}+P_{c}+P_{e x}+P_{T F}+P_{c o r r}
$$

La ecuación de estado obtenida fue usada por Hamada y Salpeter (H-S) (Hamada and Salpeter, 1961) para construir modelos a temperatura cero para las enanas blancas. Puesto que en este caso la ecuación de estado depende de $A$ y $Z$ en lugar de sólo $\mu_{e}$, es necesario especificar la composición química del modelo.

\subsection{Relaciones masa-radio para enanas blancas con diferentes composiciones internas}

Las enanas blancas obviamente no son objetos a temperatura cero, pues irradian. Las relaciones masa-radio para temperatura finita requieren cálculos evolutivos completos para 
estos remanentes que tienen en cuenta la estructura gravotérmica de la estrella. Ejemplos de relaciones masa-radio a temperatura finita son aquellos presentados por Panei y colaboradores (Panei et al., 2000b) para composiciones químicas de helio, carbono, oxígeno, silicio y hierro, con envolturas de hidrógeno y helio, y con envolturas de helio solamente.

Lo relevante de este trabajo, es que fue el primero en el que se calcularon relaciones masaradio a temperatura finita para varias composiciones químicas donde todos los resultados fueron obtenidos bajo las mismas condiciones; de manera tal que se pueden emplear un conjunto de resultados teóricos uniformes -ver por ej. (Madej et al., 2003) y (Madej et al., 2004).

Dicho trabajo fue motivado por las observaciones realizadas por el satélite Hipparcos (Provencal et al., 1998) las que sugerían la existencia de enanas blancas con núcleos de hierro. Posterior a esta publicación de Provencal y colaboradores se encontró que ellos habían empleado modelos de atmósferas equivocados, y así al corregirlos, se determinó que tales interiores estaban compuestos de carbono/oxígeno.

Se sabe que existen diferentes caminos que conducen a más del $90 \%$ de las estrellas a enanas blancas. Por ejemplo, las enanas blancas de baja masa con masas estelares de $M \lesssim$ $0.45 M_{\odot}$ están compuestas por helio y ellas han tenido tiempo suficiente para evolucionar a ese estado como resultado de la evolución binaria; ya que si hubieran evolucionado como estrellas aisladas hubieran necesitado el tiempo de Hubble para convertirse en enana blanca de helio. Para enanas blancas de masas intermedias, la teoría de evolución estelar predice una composición interna dominada por carbono y oxígeno. Finalmente, para la cola de la distribución de las enanas blancas con masas mayores, la teoría predice interiores hechos de neón y magnesio.

Es costumbre emplear relaciones masa-radio, para confrontar las predicciones teóricas sobre la composición interna de las enanas blancas con los datos observacionales. Esto es debido a que, como es bien sabido desde Hamada y Salpeter (1961), las configuraciones a temperatura cero son sensibles a la composición interna. Uno de los efectos que nos permite discriminar la composición interna de las enanas blancas para una masa estelar dada, está relacionada con la dependencia de las contribuciones no-ideales a la ecuación de estado de la materia degenerada (tal como las interacciones coulombianas y las correcciones ThomasFermi) sobre la composición química. Estas contribuciones a la ecuación de estado son mayores para los más altos números atómicos $Z$ del elemento químico. Otro efecto muy importante es que, en el caso de elementos pesados tales como el hierro, los núcleos ya no son simétricos $(Z=26, A=56$ para el hierro), dando un peso molecular medio por electrón mayor que 2 -ver Apéndice A, sec. (13.6) ec. (74) si $X_{F e}=1, Z_{F e}=26, A_{F e}=56$, entonces $\mu_{e} \simeq 2.15$. En acuerdo, para un valor dado de masa, el radio de la enana blanca es decreciente con la función $Z$. 
La idea de estrellas enanas blancas con interiores de hierro está en fuerte contradicción con las predicciones standard de los cálculos de evolución estelar, las cuales sólo permiten un interior rico en hierro en el caso de los objetos pre-supernova.

Isern y colaboradores (Isern et al., 1991) han propuesto procesos físicos capaces de formar enanas blancas de hierro. En sus cálculos encontraron que una ignición explosiva de núcleos con degeneración electrónica de $\mathrm{ONeMg}$, puede depender críticamente sobre la densidad de ignición y la velocidad del frente de quema, dando a la formación de estrellas de neutrones, supernovas termonucleares o enanas blancas de hierro.

En los cómputos realizados por Panei y colaboradores, la evolución de las estrellas enanas blancas, fue calculada para masas desde $0.15 M_{\odot}$ a $0.5 M_{\odot}$ con intervalos del $5 \%$ en masa, para el caso de núcleos de helio; de $0.45 M_{\odot}$ a $1.2 M_{\odot}$ con intervalos de $0.01 M_{\odot}$ para núcleos de carbono, oxígeno y silicio; y de $0.45 M_{\odot}$ a $1.0 M_{\odot}$ con intervalos de $0.01 M_{\odot}$ para el caso de núcleos de hierro. Las secuencias evolutivas fueron computadas hasta por debajo de una luminosidad de $\log L / L_{\odot}=-5$. Las relaciones masa-radio para enanas blancas con composiciones nucleares de ${ }^{12} \mathrm{C},{ }^{16} \mathrm{O},{ }^{28} \mathrm{Si} \mathrm{y}{ }^{56} \mathrm{Fe}$ fueron computadas para valores de temperatura efectiva, desde 5000 a $55000 \mathrm{~K}$, con pasos de $10000 \mathrm{~K}$, y desde 70000 hasta $145000 \mathrm{~K}$, con pasos de 15000K. Para el caso de los modelos de enanas blancas de helio fueron considerados valores de temperatura efectiva desde $4000 \mathrm{~K}$ hasta $20000 \mathrm{~K}$, con pasos de $4000 \mathrm{~K}$. Para explorar la sensibilidad de los modelos a una envoltura de hidrógeno, se consideraron dos valores: $M_{H} / M_{*}=10^{-5}\left(3 \times 10^{-4} M_{*}\right.$ en el caso de modelos con núcleos de helio) y $M_{H} / M_{*}=0$, es decir sin hidrógeno.

En la fig. (1) se muestran relaciones masa-radio para modelos con núcleos de helio. Se han considerado modelos con masas de hasta $0.5 M_{\odot}$; debido a que, a mayores masas los objetos podrían encender el helio durante etapas evolutivas previas y podrían no terminar sus vidas como enanas blancas de helio. Como es bien sabido los modelos con mayores radios tienen mayor temperatura efectiva. Es notable el efecto sobre el radio estelar inducido por la presencia de una envoltura externa de hidrógeno. Estos efectos son particularmente importantes para modelos de baja masa. Consideremos por ejemplo el caso de una enana blanca de helio de $0.3 M_{\odot}$. En el caso de ausencia de una envoltura de hidrógeno, para la temperatura efectiva más alta considerada en este trabajo, el objeto tiene un radio de $\approx 50 \%$ mayor que el correspondiente al modelo de H-S. Si se incluye la envoltura de hidrógeno, el radio es $\approx 80 \%$ mayor que el de H-S. Notar que a $T_{\text {eff }} \rightarrow 0$, el radio de los objetos tiende a los valores de H-S.

En las figs. (2) a (4) se muestran los resultados obtenidos para estrellas con interiores de carbono, oxígeno y silicio respectivamente. Aunque son notables, los efectos debido a temperatura finita y la presencia de una envoltura de hidrógeno, estos no son tan grandes como en el caso previo de los modelos de enanas blancas de helio, mostrados en la fig. (1). 


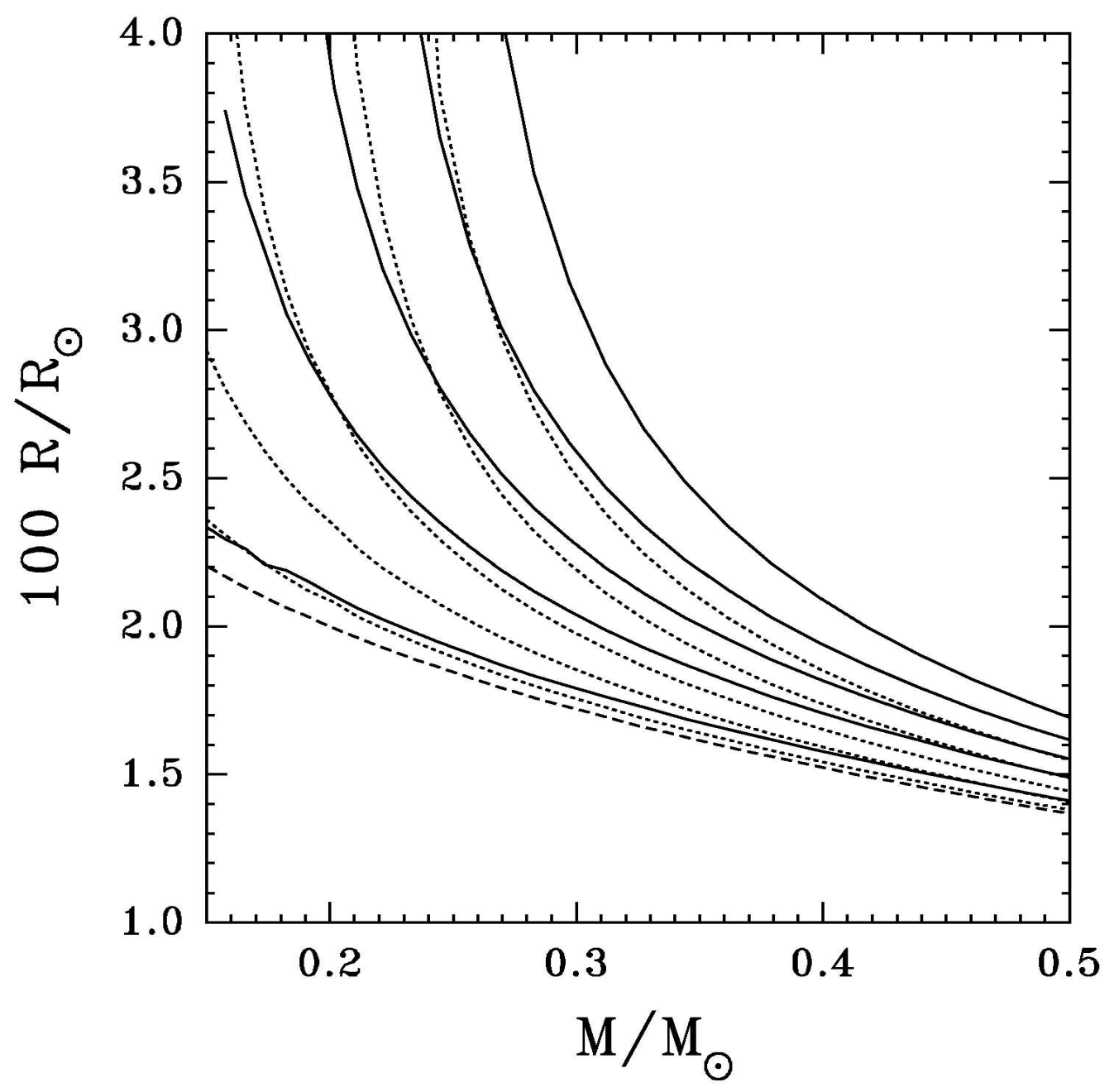

Figura 1: Relación masa-radio para enanas blancas con un núcleo de helio. Las líneas llenas y punteadas corresponden a los casos de objetos con una envoltura de hidrógeno puro de $3 \times 10^{-4} M_{*}$ y a los modelos sin esa envoltura de hidrógeno, respectivamente. Las líneas de trazos representan la relación masa-radio de $H$-S para modelos homogéneos de helio. Los modelos de temperatura finita están ordenados desde abajo hacia arriba con el aumento de temperatura $T_{\text {eff }}$ correspondiente a (en $\left.10^{3} \mathrm{~K}\right)$ 4, 8, 12, 16 y 20. 
Por ejemplo para modelos de $1.2 M_{\odot}$, ambos efectos permiten aumentar el radio de la estrella hasta $\lesssim 19 \%$. Esto es lo esperado, debido a que con el aumento de la masa, la densidad interna y el potencial químico por electrón, $\mu_{e}$, también aumenta. Esto último puede verse fácilmente pues como $\varrho \propto \frac{M}{R^{3}}$, y por la relación masa-radio -Apéndice A, sec. (13.7.3), ec. (121)- $R \propto M^{-\frac{1}{3}}$, entonces $\varrho \propto M^{2}$, y por ecs. (74) y (64) $\mu_{e} \propto \varrho$.

Así, como los efectos térmicos en la ecuación de estado del gas degenerado entran como una corrección $\propto\left(T / \mu_{e}\right)^{2}$, la ecuación de estado se vuelve más cercana a la conducta de temperatura cero, es decir a la estructura de H-S. Como el espesor de la capa de hidrógeno es $\propto g^{-1}$ (donde $g$ es la gravedad superficial) esto hace que también tienda a cero para modelos muy masivos. Notar que el carbono, oxígeno y silicio tienen peso molecular medio por electrón muy cercano a $2\left(\mu_{e}=2.001299,2.000000,1.999364\right.$ y 1.998352 para el helio, carbono oxígeno y silicio respectivamente); así, las diferencias en radio, para una dada masa estelar son en su mayoría, debido casi por completo, a los términos de corrección de la ecuación de estado para el caso no-ideal. Debido a esto las diferencias en radio son pequeñas, del orden de unos porcientos.

Se han incluido en cada figura las correspondientes secuencias de H-S. Notar que para un valor dado de la masa, los modelos $\mathrm{H}-\mathrm{S}$ tienen radios menores y que existe algunas diferencias pequeñas aún para los modelos de $T_{\text {eff }}$ más baja.

En la fig. (5) se muestran las secuencias masa-radio correspondientes a hierro. Estas son notablemente diferentes de las relaciones masa-radio mostradas anteriormente, debido principalmente a un mayor peso molecular por electrón $\left(\mu_{e}=2.151344\right)$ y también debido a un número atómico mayor $(Z=26)$ que indica un gas degenerado mucho más fuertemente interactuante, comparado con el caso de composición standard.

En el caso de un núcleo de hierro, para un valor de masa fijo, la densidad media es casi dos veces la correspondiente a la de los núcleos de carbono y oxígeno. Así, no debería sorprender que para el rango de $T_{\text {eff }}$ considerado aquí, los efectos térmicos son menos importantes que en el caso standard. Por ejemplo para el modelo de hierro de $0.45 M_{\odot}$ a $T_{\text {eff }} \approx 25000 \mathrm{~K}$, los efectos térmicos aumentan el radio sólo por un 17\%. Para el caso del núcleo de hierro, hemos considerado modelos hasta una masa de $1.0 M_{\odot}$. Objetos con masas mayores están muy próximos a la masa límite para tales composiciones $\left(M_{c h} \simeq 1.261 M_{\odot}\right.$ para el hierro de acuerdo a la ec. (19)) y podrían tener densidades internas tan altas que la ecuación de estado podría no ser apropiada. Las enanas blancas de hierro dan radios más pequeños debido a las mayores interacciones coulombianas.

Cabe mencionar que las secuencias evolutivas computadas son muy detalladas y han sido usadas recientemente en un trabajo realizado por Madej y colaboradores (Madej et al., 2003) para la búsqueda, a través de fotometría UBVRI, de objetos enanas blancas con núcleos de hierro. También Madej y colaboradores (Madej et al., 2004) han empleado nuestras relaciones 


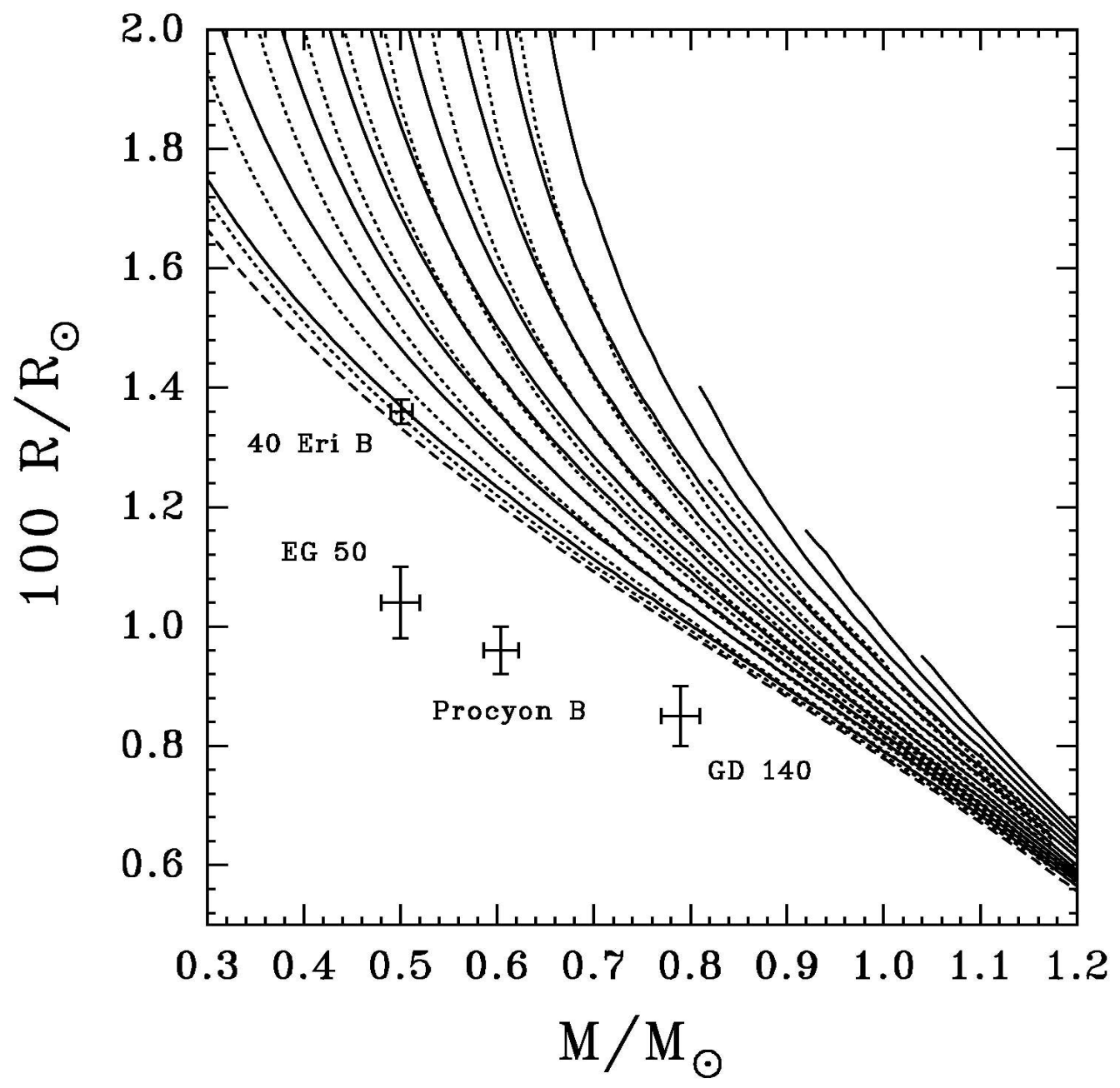

Figura 2: Relación masa-radio para enanas blancas con un núcleo de carbono rodeado por una envoltura de helio con un espesor de $10^{-2} M_{*}$. Las líneas llenas y las punteadas tienen el mismo significado que en la fig. (1) pero para el caso de la envoltura de hidrógeno asumimos una masa de $10^{-5} M_{*}$. Las líneas de trazos corresponden a la relación masa-radio de H-S para modelos homogéneos de carbono. Hemos incluido en esta figura los valores correspondientes a $T_{\text {eff }}\left(\right.$ en $\left.10^{3} \mathrm{~K}\right)$ de 5, 15, 25, 35, 45, 55, 70, 85, 100, 115, 130 y 145 . Hemos incluido datos observacionales con sus barras de error de las enanas blancas que se indican. Por completitud hemos extendido los cálculos hasta una masa de $0.3 M_{\odot}$. 


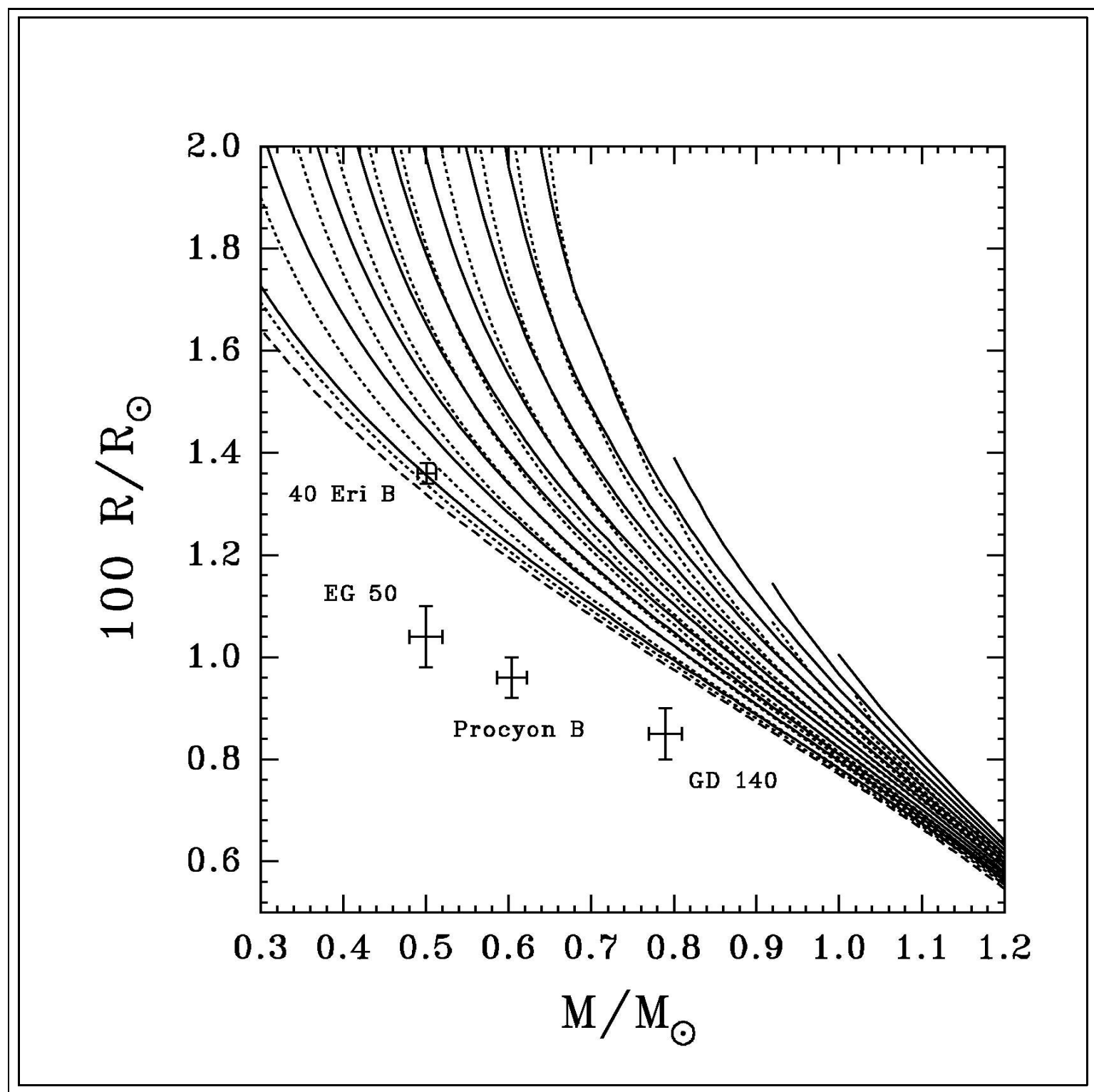

Figura 3: Igual que en la fig. (2) pero para un núcleo de oxígeno. 


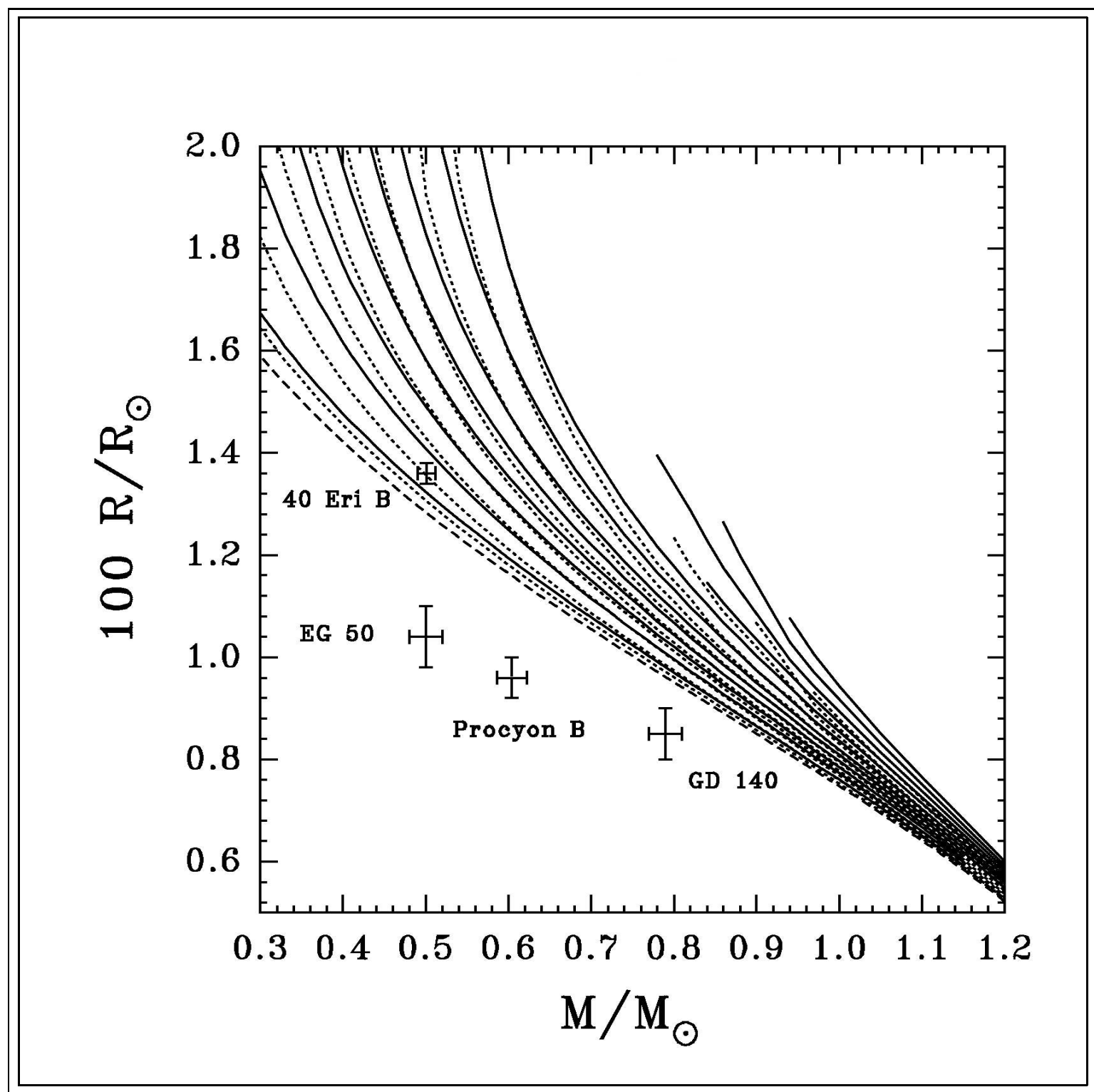

Figura 4: Igual que en la fig. (2) pero para un núcleo de silicio. 


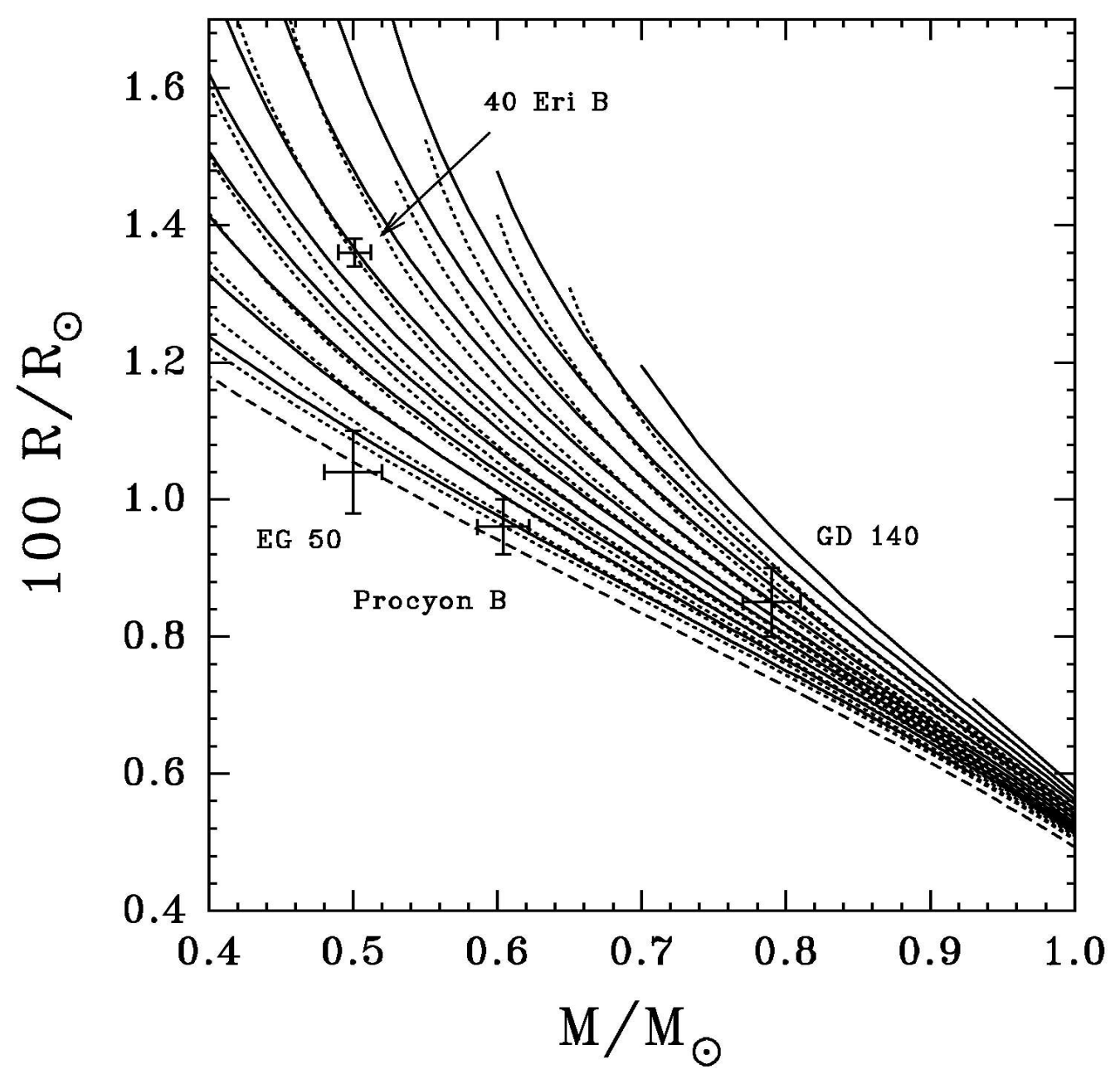

Figura 5: Igual que en la fig. (2) pero para un núcleo de hierro. Notar que en este caso los modelos resultan con radios estelares mucho menores que para las demás composiciones químicas centrales. 
masa-radio para determinar la distribución de masa de unas 1300 enanas blancas en el Sloan Digital Sky Survey.

Resultados evolutivos de las posibles enanas de hierro fueron realizados por Panei y colaboradores (Panei et al., 2000a), como así también una evaluación de la posibilidad de utilizar la función luminosidad de las enanas blancas como herramienta para discriminar entre aquellas con núcleos de hierro, de las que poseen núcleos de carbono-oxígeno. En dicho trabajo se ha examinado la evolución de las condiciones centrales, luminosidad por pérdida por neutrinos, gravedad superficial, cristalización, perfiles de luminosidad interna y edades. En estas estrellas el enfriamiento es fuertemente acelerado (hasta un factor cinco para modelos con una composición de hierro puro) comparado al del caso standard. Encontramos que las enanas blancas poseen una función luminosidad muy diferente de la que presentan las enanas blancas de carbobo-oxígeno. Este hecho permitiría en principio, diferenciar entre ambos tipos de enanas blancas. 


\section{Parte II}

\section{Formación de Estrellas Deficientes en Hidrógeno}




\section{Evolución previa a la AGB}

\subsection{Evolución hacia la fase gigante}

Las estrellas se forman a partir de la contracción del gas y polvo interestelar. Si las ubicáramos en el diagrama $\mathrm{H}-\mathrm{R}$ antes del encendido de la fuente de energía nuclear, se estarían desplazando desde la región de inestabilidad de Hayashi hacia la secuencia principal. Una vez que se inicia el quemado de hidrógeno central, se encuentran en lo que se conoce como secuencia principal. En la fig. (6), esta etapa corresponde al extremo izquierdo, el modelo representado es de una estrella con $M_{Z A M S}=5 M_{\odot}$. En la secuencia principal la estrella obtiene su energía de la quema nuclear de hidrógeno, el cual es transformado en helio, en su centro -hasta que alcanza el punto A en figs. (6), (7) y (8) ver tabla [(2), sec. (4.1)]. Debido a la gran energía ganada en la transformación de hidrógeno en helio, por ejemplo para una estrella con una masa de $1 M_{\odot}$ y con abundancias $X=0.705, Y=0.275$, la edad en la secuencia principal será de $\sim 12600 \times 10^{6}$ años (esto es cuando alcanza el punto A), para una con $1.5 M_{\odot}$ e igual abundancias será de $\sim 2600 \times 10^{6}$ años y para una de $5 M_{\odot}$ e igual abundancias será de $\sim 106.9 \times 10^{6}$ años.

\begin{tabular}{|c|c|c|}
\hline Punto & Edad $\left[10^{6}\right.$ años $]$ & Etapa evolutiva \\
\hline A & 106.926471 & agota el hidrógeno central \\
\hline B & 107.315599 & enciende el helio central \\
\hline $\mathrm{C}$ & 107.322692 & inicio del primer dredge up \\
\hline $\mathrm{D}$ & 107.551700 & fin del primer dredge up \\
\hline $\mathrm{E}$ & 122.562231 & agota el helio central \\
\hline $\mathrm{F}$ & 122.876719 & inicio del segundo dredge up \\
\hline G & 123.100129 & se acentúa el segundo dredge up \\
\hline $\mathrm{H}$ & 123.164997 & fin del segundo dredge up \\
\hline
\end{tabular}

Durante esta fase la luminosidad aumenta lentamente (hasta llegar al punto A). Cuando en la región central aproximadamente el $12 \%$ de la masa ha sido transformada en helio, comienzan a ocurrir cambios mayores en la estructura. Cuando es agotado el hidrógeno en el centro, la generación de energía nuclear (mediante quema de hidrógeno) toma lugar ahora en una capa esférica alrededor del núcleo inerte de helio (posterior al punto A y antes del B -ver fig. (8).

Este cambio es acompañado por una expansión y un enfriamiento de las capas exteriores 


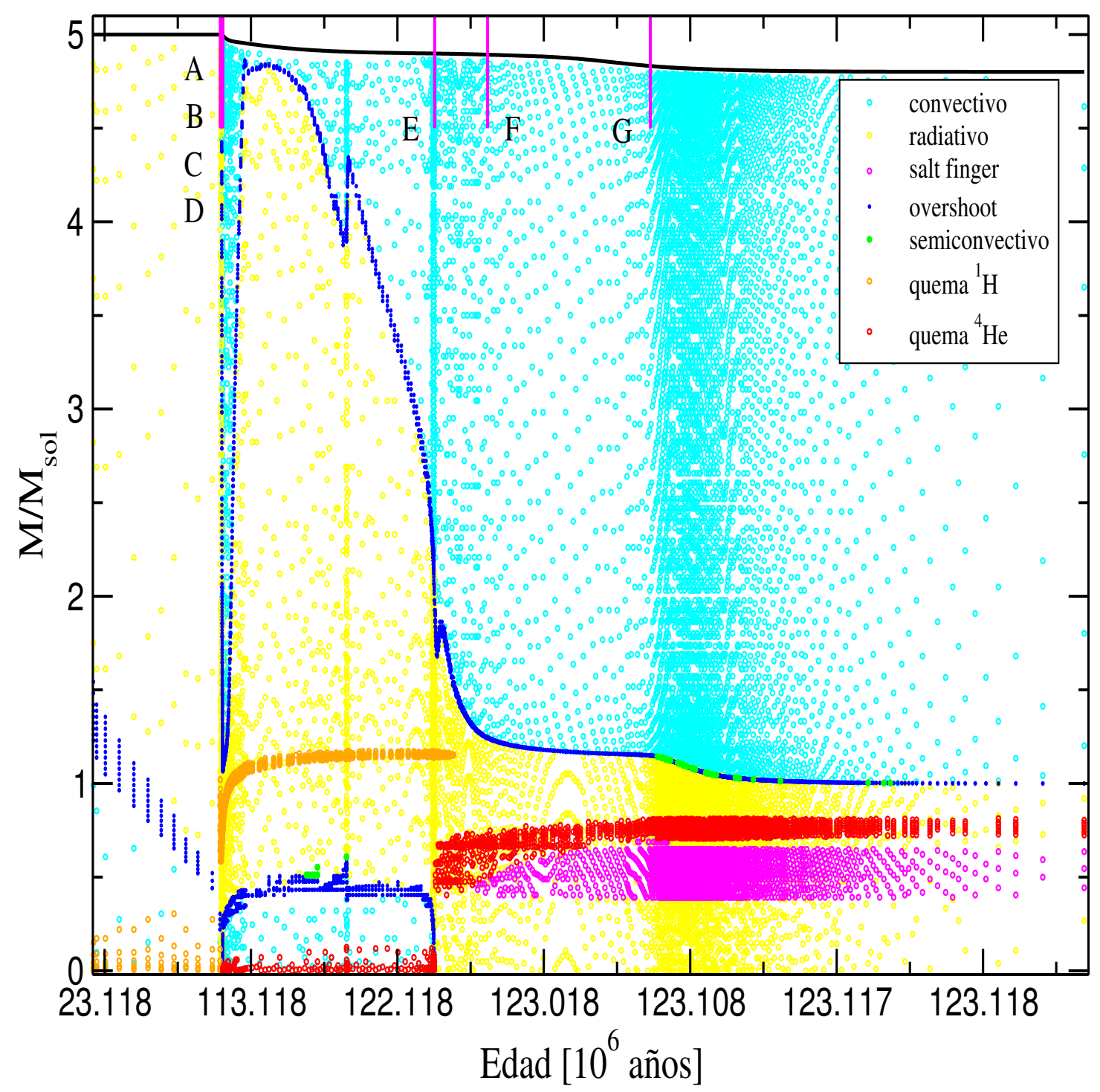

Figura 6: Interior estelar de una estrella de $M_{Z A M S}=5 M_{\odot}$, se representan los distintos transportes de energía, y las quemas de hidrógeno y helio en el interior. En ambas regiones de quema se tiene en cuenta la generación de energía nuclear $\left(\varepsilon_{H}\right.$ y $\left.\varepsilon_{H e}\right)$, cuando excede a $10^{3} \mathrm{erg} \mathrm{g}^{-1} \mathrm{~s}^{-1}$. Obsérvese la disminución de la masa estelar total (la línea llena que comienza en $M / M_{\odot}=5$ ), en este caso la pérdida de masa la iniciamos, en el código de evolución, cuando la estrella trepa por la rama gigante. 


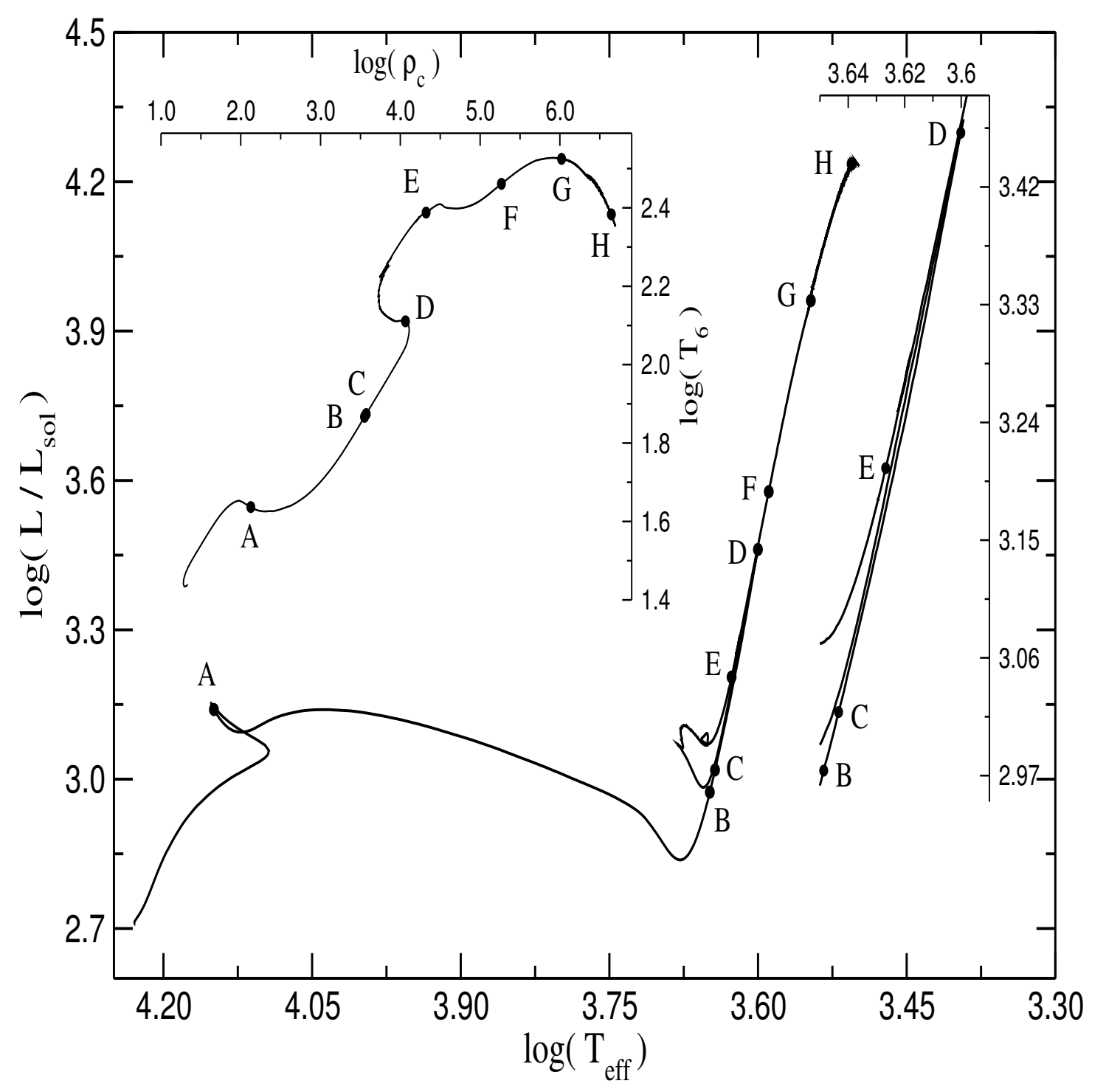

Figura 7: Trayectoria evolutiva de una estrella de $M_{Z A M S}=5 M_{\odot}$, diagrama $H-R$ y evolución de su región central. A la derecha graficamos la rama gigante en forma ampliada, para ver con más detalle los puntos dados en la tabla [(2), sec. (4.1)]. 
de la estrella: en el diagrama $\mathrm{H}-\mathrm{R}$ la estrella se mueve a la derecha y arriba hacia radios mayores (antes de arribar el punto B en el rojo, ver fig. (7)). Mientras la estrella se va desplazando del punto $\mathrm{B}$ al $\mathrm{D}$, está recorriendo la parte de la trayectoria conocida como rama gigante roja (RGB: Red Giant Branch).

\section{2 $1^{\text {er }}$ dredge up}

Desde el punto de vista de las comparaciones con las observaciones, los cambios de abundancia superficial que ocurren, previo a la fase AGB, son de gran importancia. Estos cambios son realizados cuando la base de la envoltura convectiva se extiende hacia adentro para dragar el material que ha experimentado la quema de hidrógeno durante las fases precedentes. La representación del interior de tal etapa evolutiva se realiza en la fig. (8). El $1^{\mathrm{er}}$ dredge up o $1^{\mathrm{er}}$ mezclado convectivo externo (MCE), ocurre en la estrella cuando se convierte en gigante roja por primera vez (siguiendo al agotamiento de hidrógeno central). Para una estrella de $5 M_{\odot}$ los mayores resultados que obtuvimos, son los siguientes, ver fig. (10): aumento de $\approx 180 \%$ más para la abundancia superficial del ${ }^{14} \mathrm{~N}$, una reducción en la abundancia superficial del ${ }^{12} \mathrm{C}$ de $\approx 30 \%$, la formación de la razón superficial ${ }^{12} \mathrm{C} /{ }^{13} \mathrm{C}$ de alrededor $22\left(0.205 \times 10^{-02} / 0.934 \times 10^{-04}\right.$ hacia el fin del $\left.1^{e r} \mathrm{MCE}\right)$, una reducción en la superficie del ${ }^{7} \mathrm{Li}\left(0.123 \times 10^{-09}-0.732 \times 10^{-10}\right.$ valores al inicio y al fin del $\left.1^{\text {er }} \mathrm{MCE}\right)$, una reducción de $\approx 4 \%$ en el ${ }^{1} \mathrm{H}$ y $\approx 9 \%$ en el ${ }^{16} \mathrm{O}$, y un aumento de $\approx 9 \%$ para el ${ }^{4} \mathrm{He}$.

\subsection{Evolución en la RGB}

En la parte superior de la RGB (Red Giant Branch) en el punto D, la estrella alcanza en su centro las condiciones necesarias para encender el helio y comenzar los próximos procesos nucleares, para transformarlo en carbono, oxígeno y neón.

\subsubsection{Estrellas de baja masa}

En las estrellas de baja masa como el sol, la densidad en el centro en la fase RGB, es tan alta que los electrones están parcialmente degenerados. En la materia no-degenerada, el aumento de temperatura causado por la nueva fuente de energía, podría estar acompañado por un aumento de presión, conduciendo a la expansión, enfriamiento y estabilización de la tasa de quema de helio. Sin embargo, debida a la degeneración, no hay cambios significativos en la presión; el aumento de temperatura conduce a una nueva liberación de energía y desarrollando una inestabilidad térmica. Este fenómeno es llamado "flash de helio"; conduce a una reestructuración dinámica de la estrella después de que la degeneración es disipada a altas temperaturas. 


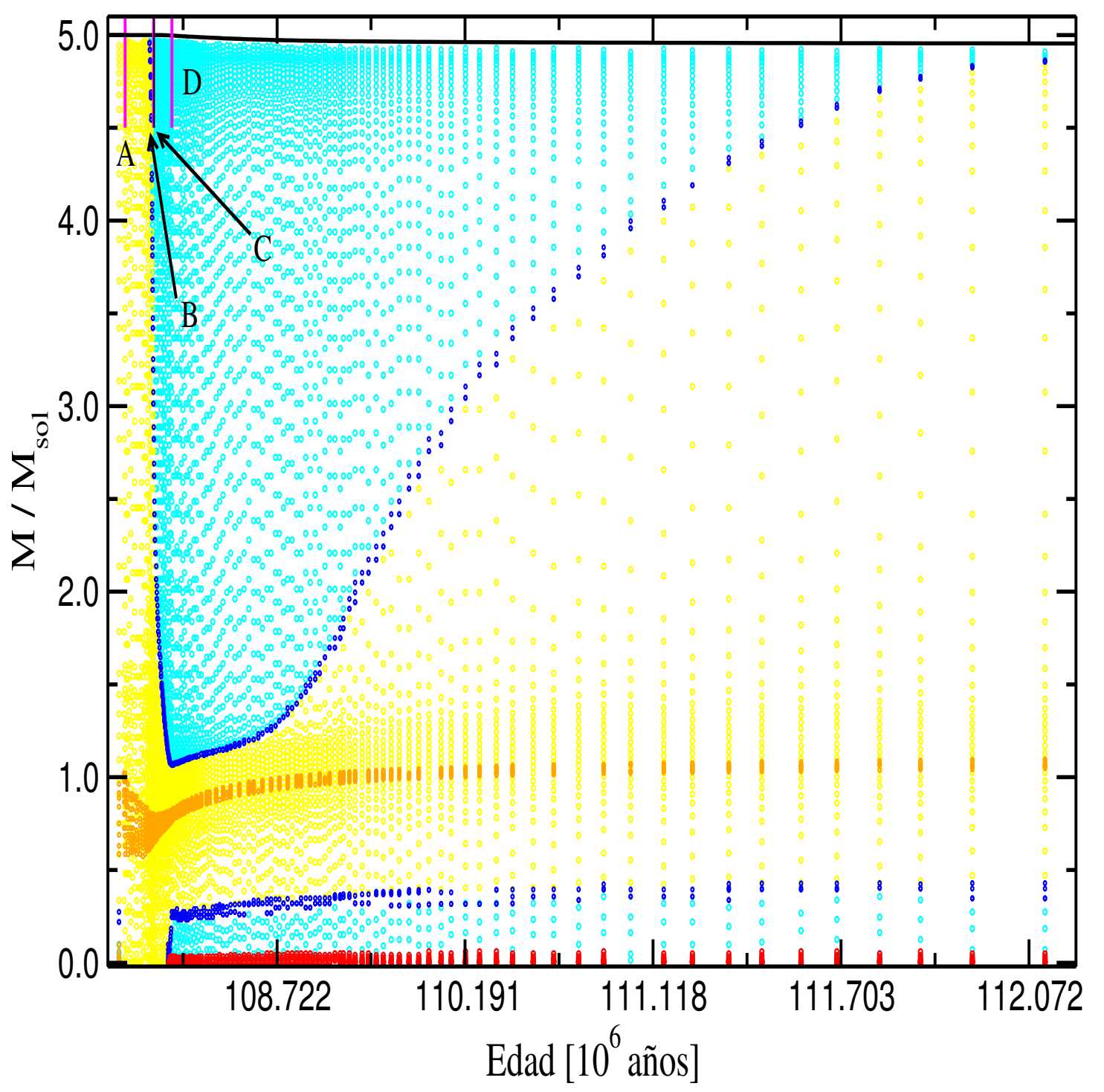

Figura 8: Interior estelar de una estrella de $M_{Z A M S}=5 M_{\odot}$, durante el $1^{\text {er }}$ "dredge up". Representamos los distintos transportes de energía y la quema de hidrógeno y helio en el interior, para el caso en que las generaciones de energía nuclear $\varepsilon_{H}$ y $\varepsilon_{H e}$ exceden a $10^{3} \operatorname{erg~g}^{-1} s^{-1}$. 


\subsubsection{Estrellas de masa intermedia}

En estas estrellas, la expansión de las capas exteriores transforman a las mismas en gigante roja lo cual sucede en una etapa muy corta de su evolución. En este momento de la evolución la estrella se mueve del punto B al D.

La evolución es similar cualitativamente para todas las estrellas en las cuales el helio se enciende antes de que el núcleo se vuelva degenerado.

Como consecuencia de la rápida contracción y calentamiento del núcleo, se inicia la quema de helio en el centro (punto B). La estrella se encuentra entonces en la región de las gigantes rojas. Luego de esta etapa, cuando desciende por la rama gigante, ésta se vuelve hacia la izquierda sobre el diagrama $\mathrm{H}-\mathrm{R}$, para realizar un loop en su trayectoria (para $3.64 \lesssim \log T_{\text {eff }} \lesssim 3.68$, de la fig. (7)). El helio es quemado en el núcleo y llega a su punto más azul, cuando éste tiene una abundancia de $Y \approx 0.66$. Posteriormente se desplaza nuevamente hacia el rojo hacia la vecindad de la línea de Hayashi. El tamaño de los loops realizados depende de la masa de la estrella (existe una variación también debido al overshoot y las tablas de opacidades empleadas).

En el núcleo la quema de helio cesa cuando el ${ }^{4} \mathrm{He}$ es convertido completamente en ${ }^{12} \mathrm{C}$, ${ }^{16} \mathrm{O}$ y ${ }^{20} \mathrm{Ne}$ (punto E). La quema continúa en una capa concéntrica rodeando al núcleo agotado (ésta se inicia poco después del punto E). Mientras el helio se quema en una capa y hacia afuera en masa, el núcleo de carbono-oxígeno aumenta en masa, gracias a las cenizas de la capa que quema helio, y se contrae. La estrella tiene dos fuentes de energía, la capa que quema helio y otra capa por encima de ésta y por debajo de la envoltura que quema hidrógeno. Se produce en este momento una contracción del núcleo, una expansión de la región con helio entre ambas fuentes y una contracción de la envoltura. Baja la temperatura de la capa que quema hidrógeno y por lo tanto cesa la quema. Ahora la contracción del núcleo es acompañada por una expansión de las capas que están por encima de la quema de helio.

La envoltura convectiva externa, gradualmente se mueve hacia abajo en extensión hasta alcanzar más del $80 \%$ de la masa estelar. Los núcleos que han sido procesados en elementos más pesados son ahora dragados hacia la superficie. Este fenómeno es conocido como el $2^{\text {do }}$ dredge up o $2^{\mathrm{do}}$ mezclado convectivo externo (MCE).

\section{4 $2^{\text {do }}$ dredge up}

La fase del $2^{\text {do }}$ mezclado convectivo externo (MCE) ocurre en las estrellas de masa intermedia más masivas, y acompaña a la formación de un núcleo con electrones degenerados a continuación del agotamiento de helio central.

En el material dragado, el cual puede ser tan grande como $1 M_{\odot}$ en las estrellas de masa 


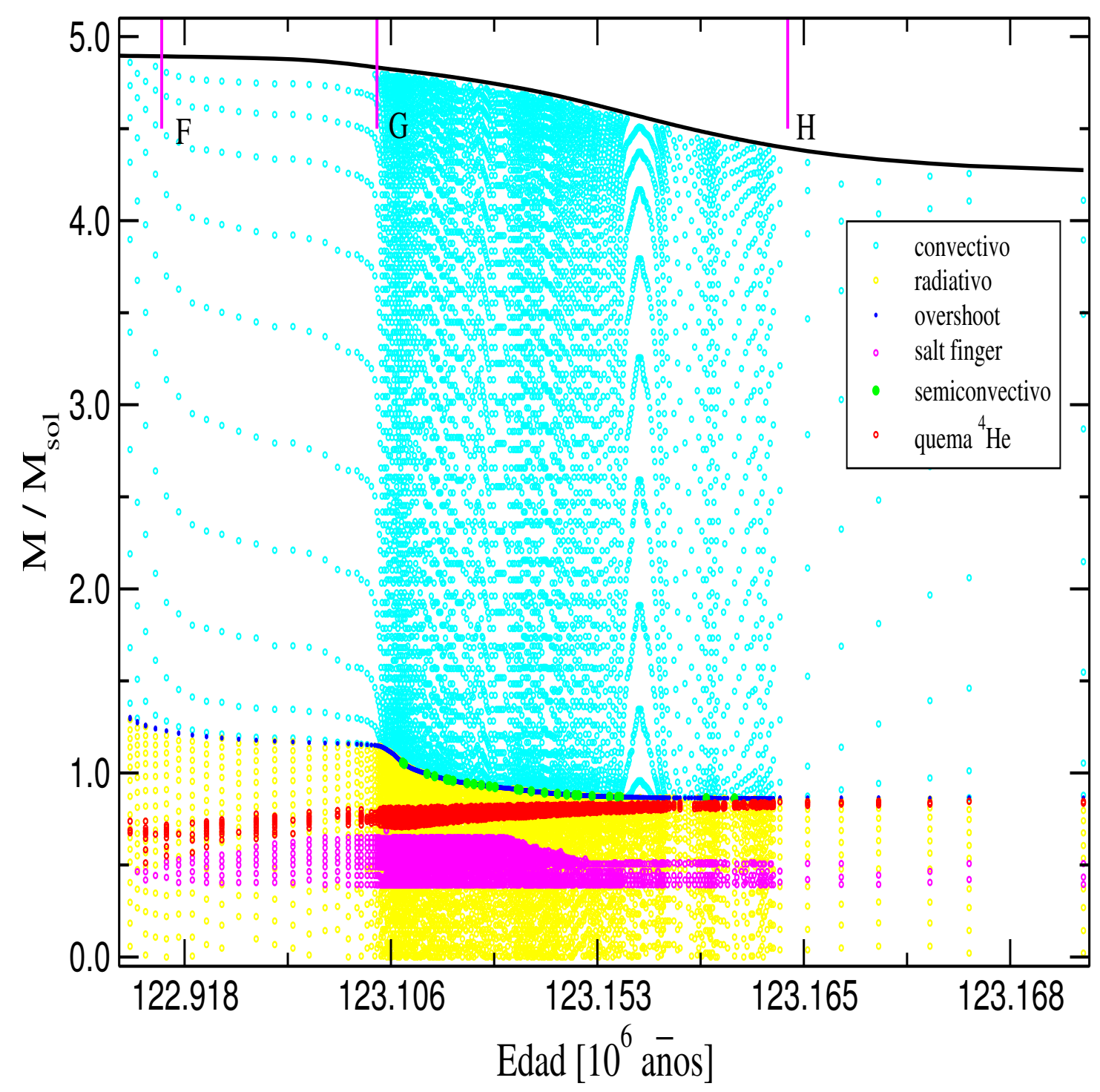

Figura 9: Interior estelar de una estrella de $M_{Z A M S}=5 M_{\odot}$, durante el $2^{\text {do }}$ "dredge up", para el caso en que las generaciones de energía nuclear $\varepsilon_{H}$ y $\varepsilon_{H e}$ exceden a $10^{3} \mathrm{erg} \mathrm{g}^{-1} \mathrm{~s}^{-1}$. 


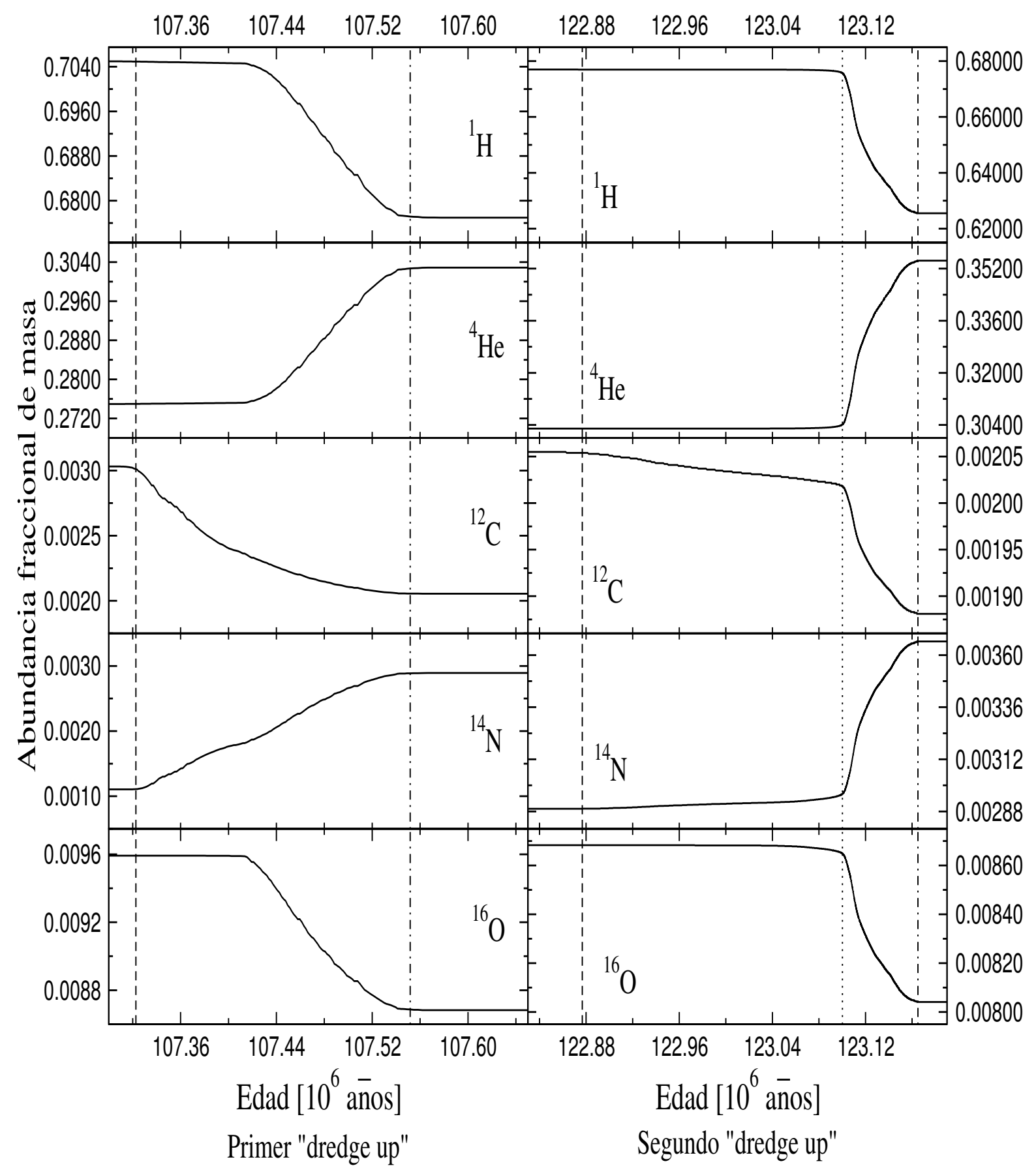

Figura 10: Cambios de abundancias superficiales durante el $1^{\text {er }}$ y $2^{\text {do }}$ "dredge up", para una estrella $M_{Z A M S}=5 M_{\odot}$. Para las curvas representadas a la izquierda, la línea de segmentos indica el comienzo del $1^{\text {er }}$ "dredge up" (punto C) y la correspondiente de punto y segmentos el fin del mismo (punto D). En las curvas de la derecha las líneas de segmentos y de segmentos y puntos tienen el mismo significado anterior, pero para el $2^{\text {do }}$ "dredge up" (puntos F y H). La línea de puntos es la que corresponde al punto $G$, que es durante el momento en que se va acentuando el $2^{\text {do }}$ "dredge up'. 
intermedia más masivas, el hidrógeno ha sido convertido completamente en helio y ambos el ${ }^{12} \mathrm{C}$ y ${ }^{16} \mathrm{O}$ han sido convertidos casi completamente en ${ }^{14} \mathrm{~N}$. En la fig. (9) ilustramos como la base de la envoltura convectiva se mueve hacia abajo en masa a través de la materia que está siendo dragada hacia afuera desde la capa que quema helio durante la fase del $2^{\mathrm{do}} \mathrm{MCE}$ (punto G).

En esta fase la temperatura en el núcleo agotado en helio y en rápida contracción, va primero en aumento como resultado de la liberación de energía potencial gravitacional y luego decrece cuando se vuelve importante la liberación de energía por neutrinos. El resultado neto es un núcleo con electrones altamente degenerados en el cual la temperatura no se vuelve lo suficientemente alta como para que se encienda el carbono.

\subsection{Evolución durante la AGB temprana}

De acuerdo a lo visto en la sec. (4.3) las estrellas de baja masa desarrollan un núcleo de helio con degeneración electrónica, inmediatamente después de la etapa de secuencia principal. Estas estrellas tienen, como límite inferior para la masa, de $0.8-1 M_{\odot}$ de masa inicial en la secuencia principal y como límite superior de $2-2.3 M_{\odot}$; la precisión de los límites depende de la composición química inicial.

Las estrellas de masa intermedia son aquellas que encienden el helio en forma "nodegenerada", pero desarrollan un núcleo de carbono-oxígeno con degeneración electrónica seguido del agotamiento de helio en el núcleo. Estas tienen como límite superior alrededor de $7-8 M_{\odot}$ como masa inicial en la secuencia principal, nuevamente el valor preciso depende de la composición química inicial.

Las estrellas de baja masa también desarrollan un núcleo de carbono-oxígeno con degeneración electrónica después de agotar el helio central; durante su evolución posterior tienen una conducta cualitativamente similar a las estrellas de masa intermedia para los mismos estados evolutivos nucleares. Esta fase común se refiere a la fase evolutiva de la rama asintótica gigante (AGB).

La AGB temprana o E-AGB (early-AGB) es aquella en la cual las estrellas no han comenzado a pulsar térmicamente. En la fase de la E-AGB, la quema de hidrógeno en capa se ha extinguido, y el helio se quema en una delgada capa que provee la mayoría de la energía que alcanza la superficie estelar (desde el punto $\mathrm{F}$ y hasta antes de los pulsos térmicos).

La designación "asintótica" en las siglas AGB proviene del hecho que, para masas iniciales $M_{Z A M S} \lesssim 1 M_{\odot}$, la relación $T_{\text {eff }}-L$ para las estrellas AGB de baja masa es muy próxima a la relación $T_{\text {eff }}-L$ para las de baja masa de la primer rama gigante. Para las estrellas más masivas el término "asintótico" no tiene sentido morfológico.

Hacia el final de la fase E-AGB, el hidrógeno se reenciende en una capa delgada y la 
estrella comienza a pulsar térmicamente. La ubicación en masa de la capa que quema hidrógeno $\left(M_{H}\right)$ cuando comienza el pulso térmico, es una función bien definida de la masa inicial $M_{Z A M S}$. Para estrellas de masa intermedia con composición similar a las estrellas de Población I, esta masa está dada aproximadamente por $M_{H} \simeq 0.59+0.0526 M_{Z A M S}$ (según Iben, para el caso en que $M_{Z A M S}=5 M_{\odot}$, es $\simeq 0.853$, según nuestros cálculos este valor es $\simeq 0.8638$ ). Para estrellas de baja masa, $M_{H}$ es sólo escasamente mayor que la masa del núcleo de hidrógeno agotado, hacia el encendido del flash de helio, o $M_{H} \simeq 0.53$ (Iben and Renzini, 1983). 


\section{Evolución durante la AGB}

Las estrellas de masa intermedia evolucionan después de completar la quema de hidrógeno y helio central, a través de la fase AGB. En esa etapa, consisten en un objeto compacto con un núcleo de carbono/oxígeno $\left(\sim 0.5, \ldots, 1 M_{\odot}\right)$ rodeado por una delgada capa de algunas $10^{-2} M_{\odot}$ ocupadas por dos envolturas que queman helio e hidrógeno respectivamente, y una enorme envoltura casi totalmente convectiva. Aunque la fase AGB es corta, es de esencial importancia puesto que está gobernada por una rica nucleosíntesis. Se suceden procesos, como los de mezcla, los cuales llevan hacia la superficie de la estrella, el material producido en el interior, y fuertes vientos estelares que "barren" las superficies estelares, por lo que continuamente enriquecen al medio interestelar con elementos pesados. Esto importa en la evolución en la parte superior de la AGB la cual es dominada por la continua y creciente pérdida de masa (Habing, 1996). Las observaciones indican tasas de $10^{-7} M_{\odot} /$ año para estrellas Mira de cortos períodos y hasta $10^{-4} M_{\odot} / a \tilde{n} o$ para estrellas variables luminosas de largo período (Wood, 1997). Muy probablemente, estos vientos son conducidos por polvo y ondas de choques (Winters, 1998) llevando grandes tasas de pérdida de masa hasta el completo ocultamiento de la estrella por una envoltura circunestelar de polvo.

Los procesos de mezcla y nucleosíntesis, las inestabilidades térmicas de la quema de helio en capas (pulsos térmicos) y la posible penetración de la envoltura convectiva dentro de la capa de quema de hidrógeno ( $H B B$ : Hot Bottom Burning) son cruciales para la evolución en la parte superior de la AGB.

\subsection{Pulsos térmicos y $3^{\text {er }}$ mezclado convectivo externo}

Los pulsos térmicos ocurren en modelos que contienen una o más capas que son fuente de energía. La inestabilidad se manifiesta en una corrida térmica: la capa reacciona al excedente de energía con un aumento de la temperatura, el cual incrementa la liberación de energía nuclear, etc. El aumento de temperatura está conectado a una expansión de las capas. Puesto que la quema de helio es extremadamente sensible con la temperatura -ver ec. (131)-, un pequeño aumento de la temperatura incrementa en gran parte la producción de luminosidad debida al helio en la capa. Esta enorme cantidad de energía fuerza al desarrollo de una zona convectiva conductora de los pulsos, la cual mezcla los productos de la quema de helio, esto es carbono y oxígeno, dentro de una región intercapa (que está ubicada por encima de ella y por debajo de la envoltura convectiva externa). Pero la mayor parte de la energía es usada para expandir las capas de arriba, empujando al material dentro de regiones más frías. Esta expansión reduce considerablemente la temperatura de la capa fuente de energía debida a la quema de hidrógeno, tal que la luminosidad debida a la quema de hidrógeno 
se reduce significativamente. Después de su comienzo aproximadamente lento, la corrida térmica se acelera más y más, hasta alcanzar un pico marcado en pocos años. La capa de helio ahora está muy expandida y entonces deja de ser inestable. Toda la región entonces comienza a contraerse de nuevo, lo cual calienta la capa de hidrógeno y entonces se recupera la productividad de energía. En unos pocos miles de años la región recupera su estructura original, la capa de helio se vuelve nuevamente inestable y comienza el próximo pulso.

Cuando la quema de hidrógeno cesa temporariamente, permite a la envoltura convectiva avanzar hacia abajo después del pulso, para penetrar en aquellas regiones de la intercapa enriquecidas anteriormente con carbono (y oxígeno) y así mezclar este material hacia la superficie. Con el avance de la envoltura convectiva hacia abajo, posterior al pulso, se produce lo que se conoce como $3^{\text {er }}$ dredge up, el cual es el encargado de llevar a la formación de estrellas de carbono. Al igual que antes este dragado (que se sucede en los pulsos térmicos y por lo tanto más de una vez) es el encargado de enriquecer nuevamente la superficie con elementos pesados.

En la parte superior de la AGB la quema de helio en capas se vuelve recurrentemente inestable creciendo hacia los pulsos térmicos [(Schwarzschild and Härm, 1965);(Weigert, 1966)]. Durante estas inestabilidades la luminosidad de la capa de helio aumenta rápidamente en un tiempo corto, por ejemplo para una estrella de $M_{Z A M S}=2.5 M_{\odot}$, en unos 200 años va desde $10^{3}$ a $10^{7} L_{\odot}$. La fig. (11) muestra la evolución de las luminosidades de la quema en capa de hidrógeno y helio durante los pulsos térmicos de una estrella de $M_{Z A M S}=2.5 M_{\odot}$. Computado con el código de evolución estelar.

La zona convectiva conductora de los pulsos puede verse en las figs. (12), (13), (14) y (15). En la fig. (15) se observa claramente que una pequeña región de la masa estelar $\left(\Delta m_{r} \simeq 0.02 M_{\odot}\right)$ es la encargada de soportar la envoltura de la estrella que al inicio de los pulsos térmicos es de $\simeq 1.8 M_{\odot}\left(\right.$ para $\left.M_{Z A M S}=2.5 M_{\odot}\right)$.

Existe una gran liberación de energía en la base de la capa convectiva en donde se realiza la quema de helio -ver fig. (15). Debido a que la capa de hidrógeno es empujada dentro de regiones más frías, la quema de hidrógeno cesa temporariamente, de esta manera la envoltura convectiva avanza hacia abajo después del pulso, para penetrar en aquellas regiones intercapas enriquecidas anteriormente con carbono (y oxígeno) y llevar este material hacia la superficie -ver fig. (14). El cambio en las abundancias se observa en la fig. (16).

Luego la quema nuclear debida al pulso de hidrógeno se reinicia y provee nuevamente la principal fuente de energía. 


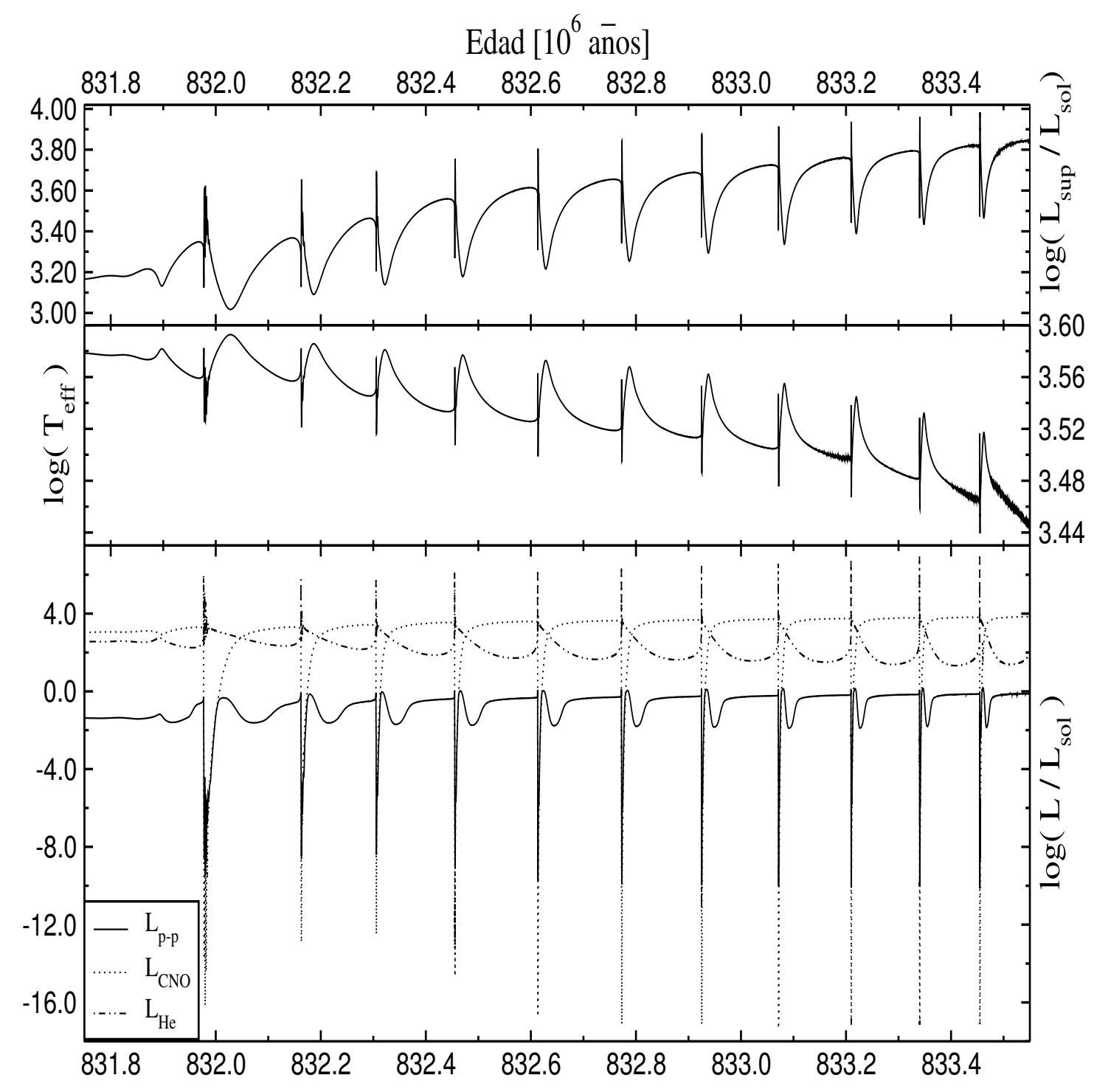

Figura 11: Luminosidad superficial, temperatura efectiva, la luminosidad debido a la quema de hidrógeno por el ciclo p-p y CNO, y la quema de helio; para una estrella de $M_{Z A M S}=2.5 M_{\odot}$. 


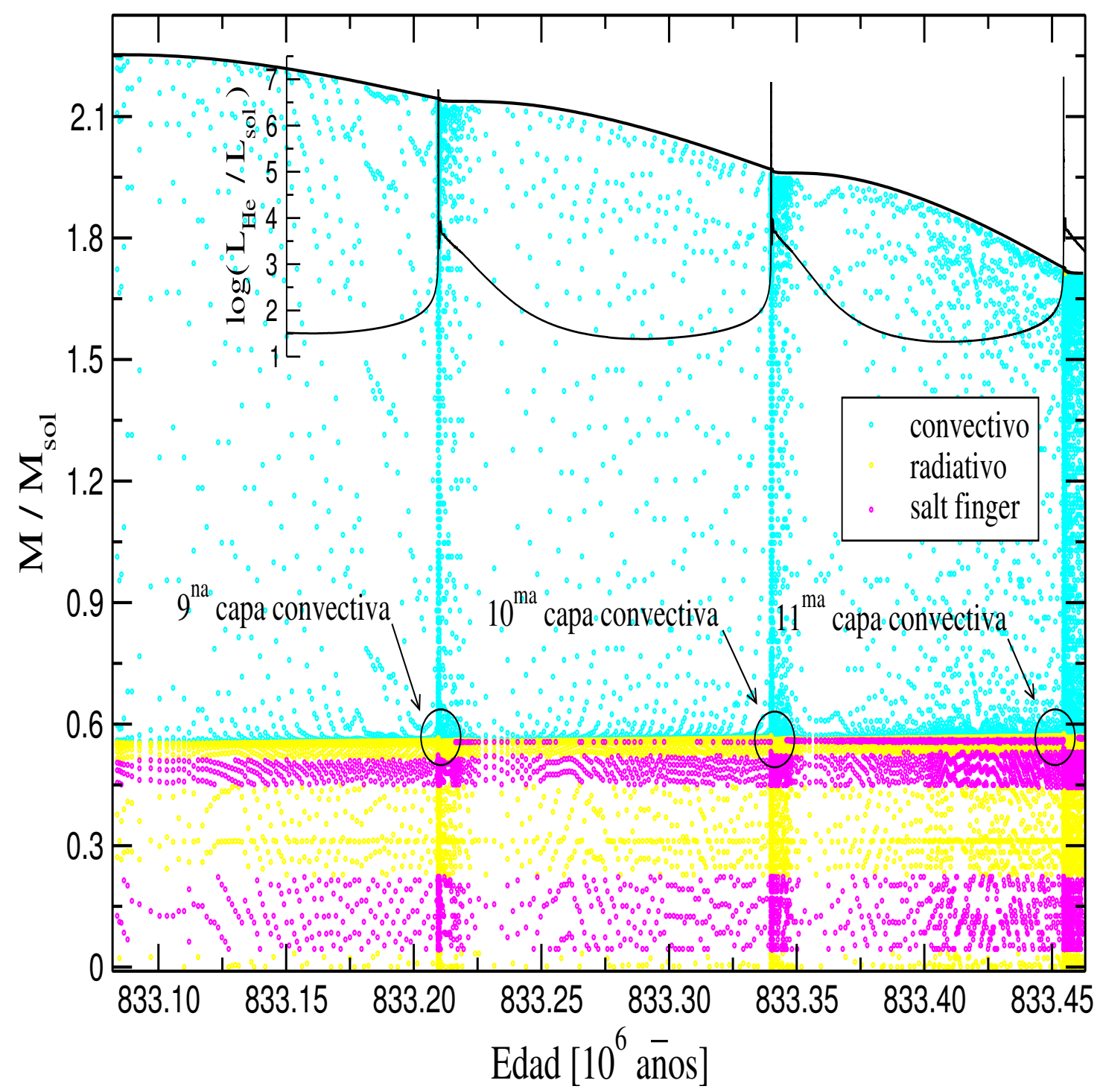

Figura 12: Interior de una estrella con una masa de $M_{Z A M S}=2.5 M_{\odot}$, durante el $9^{\text {no }}, 10^{\text {mo }}$ y $11^{\text {mo }}$ pulso. Se indican distintos tipos de transporte y también la luminosidad integrada debida a la quema de helio. En la figura se ha agregado en qué lugar debajo de la envoltura convectiva, se forma la capa conductora de los pulsos. Notar que esta es una región diminuta de la estrella, ya que la misma es $\Delta m_{r} \simeq 0.02 M_{\odot}$. 


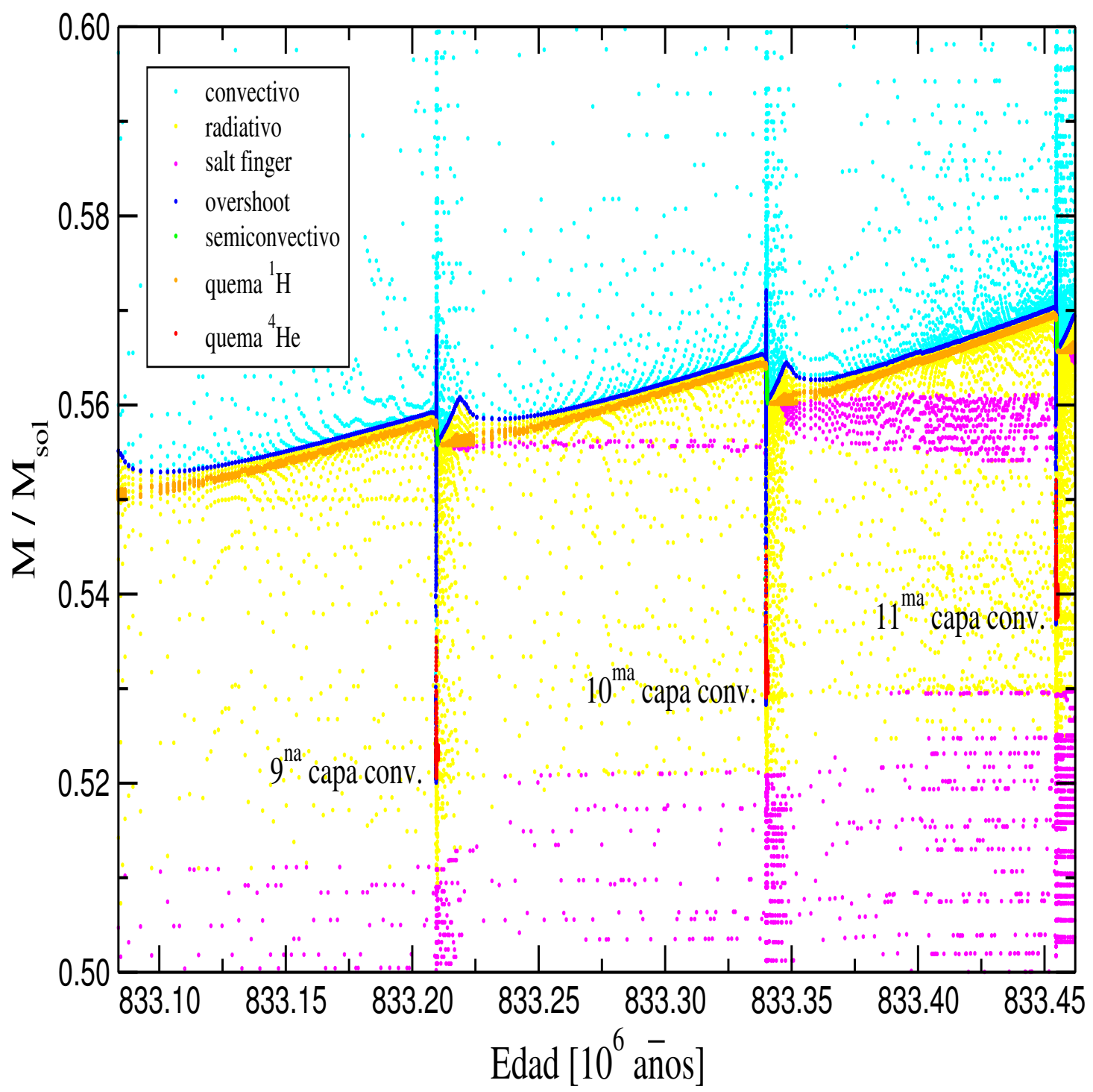

Figura 13: Interior de una estrella con una masa de $M_{Z A M S}=2.5 M_{\odot}$, durante el $9^{\text {no }}, 10^{\text {mo }}$ y $11^{\text {mo }}$ pulso. Se muestra la región correspondiente donde se encuentra la capa convectiva. Se indican distintos tipos de transporte y también la quema de hidrógeno y helio. Se ha considerado liberación de energía cuando $\varepsilon_{H}>10^{3} \mathrm{erg}^{-1} \mathrm{~s}^{-1}$ y $\varepsilon_{H e}>3 \times 10^{6} \mathrm{erg}^{-1} \mathrm{~s}^{-1}$. 


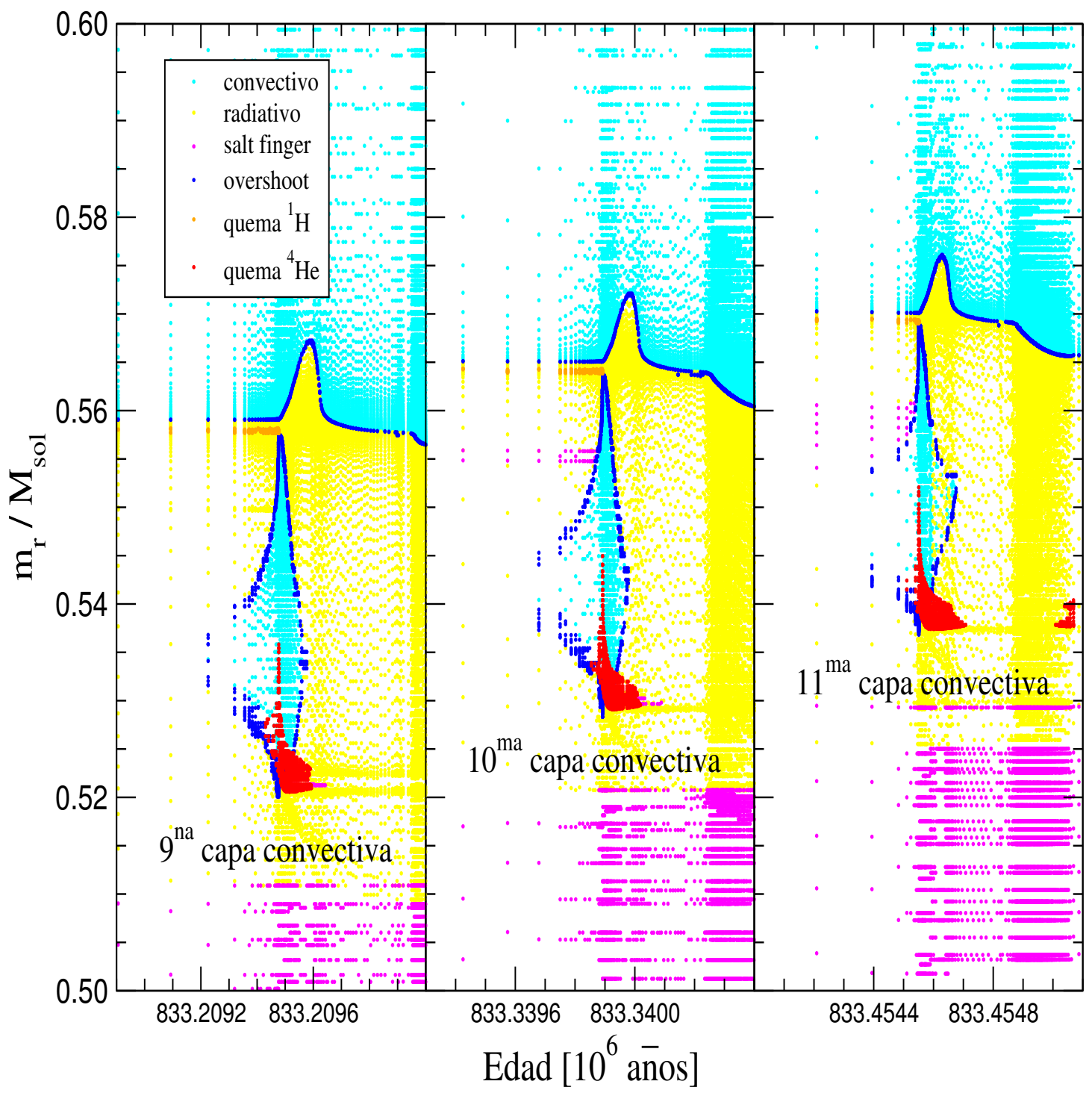

Figura 14: Interior de una estrella con una masa de $M_{Z A M S}=2.5 M_{\odot}$, durante el $9^{\text {no }}$, $10^{\text {mo }}$ y $11^{\text {mo }}$ pulso. Se muestran en forma ampliada las capas convectivas, los distintos tipos de transporte y también la quema de hidrógeno y helio. En la figura se muestra como cesa la quema de hidrógeno debido a que la capa de hidrógeno es empujada hacia afuera de la estrella hacia regiones más frías. Notar que posteriormente la envoltura convectiva avanza hacia adentro para dragar el helio, carbono y oxígeno hacia la superficie. Se ha considerado liberación de energía cuando $\varepsilon_{H}>10^{3} \mathrm{erg} \mathrm{g}^{-1} \mathrm{~s}^{-1}$ $y \varepsilon_{H e}>3 \times 10^{6} \mathrm{erg}^{-1} \mathrm{~s}^{-1}$. 


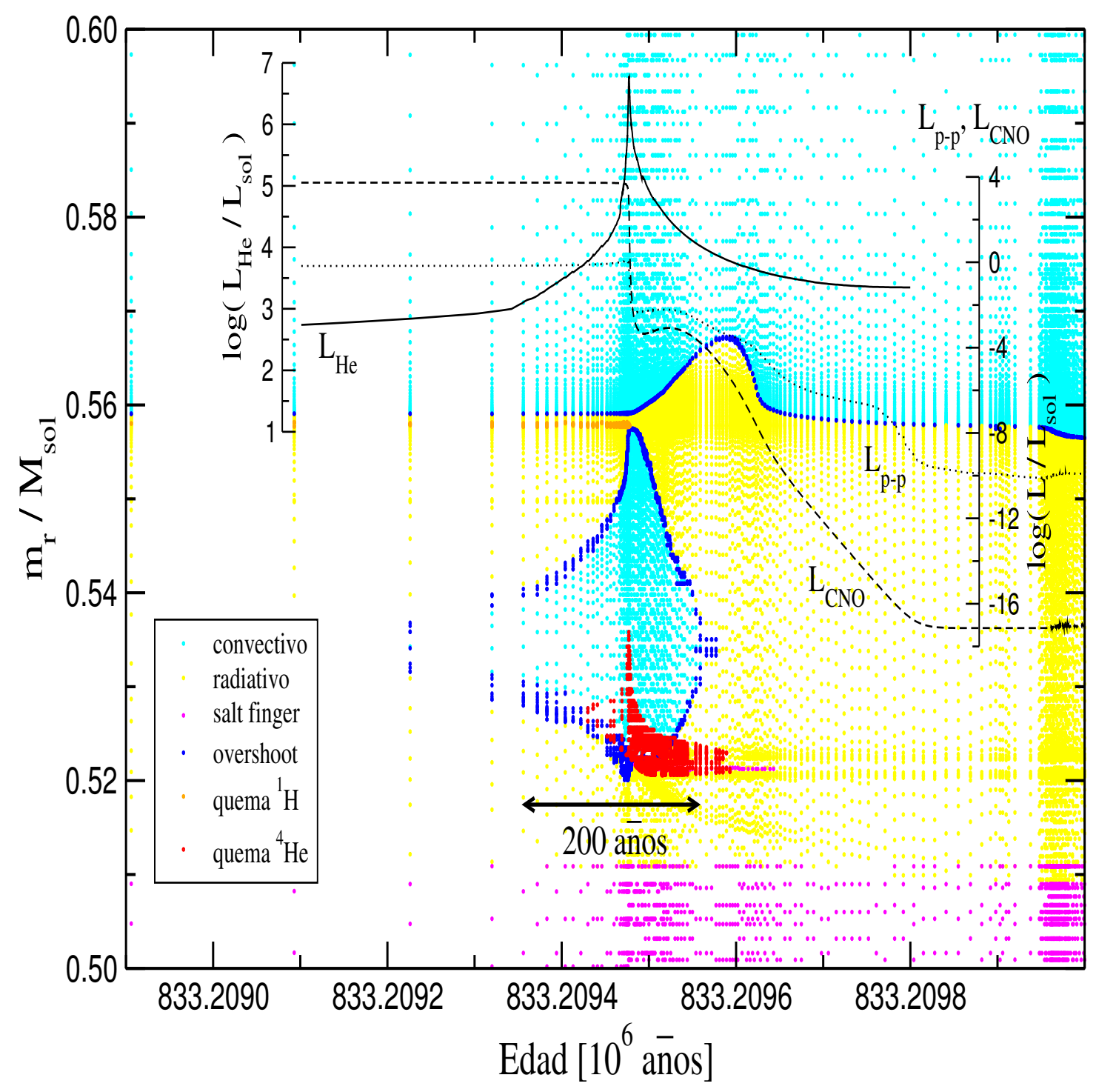

Figura 15: Interior de una estrella con una masa de $M_{Z A M S}=2.5 M_{\odot}$, durante el $9^{\text {no }}$ pulso. Se muestran los distintos tipos de transporte y la quema de hidrógeno y helio. Se indican las luminosidades integradas de la quema de hidrógeno por los ciclos p-p y CNO, y la correspondiente a la de helio. Se ha considerado liberación de energía cuando $\varepsilon_{H}>10^{3} \mathrm{erg} \mathrm{g}^{-1} \mathrm{~s}^{-1}$ y $\varepsilon_{H e}>$ $3 \times 10^{6} \mathrm{erg} \mathrm{g}^{-1} \mathrm{~s}^{-1}$. 


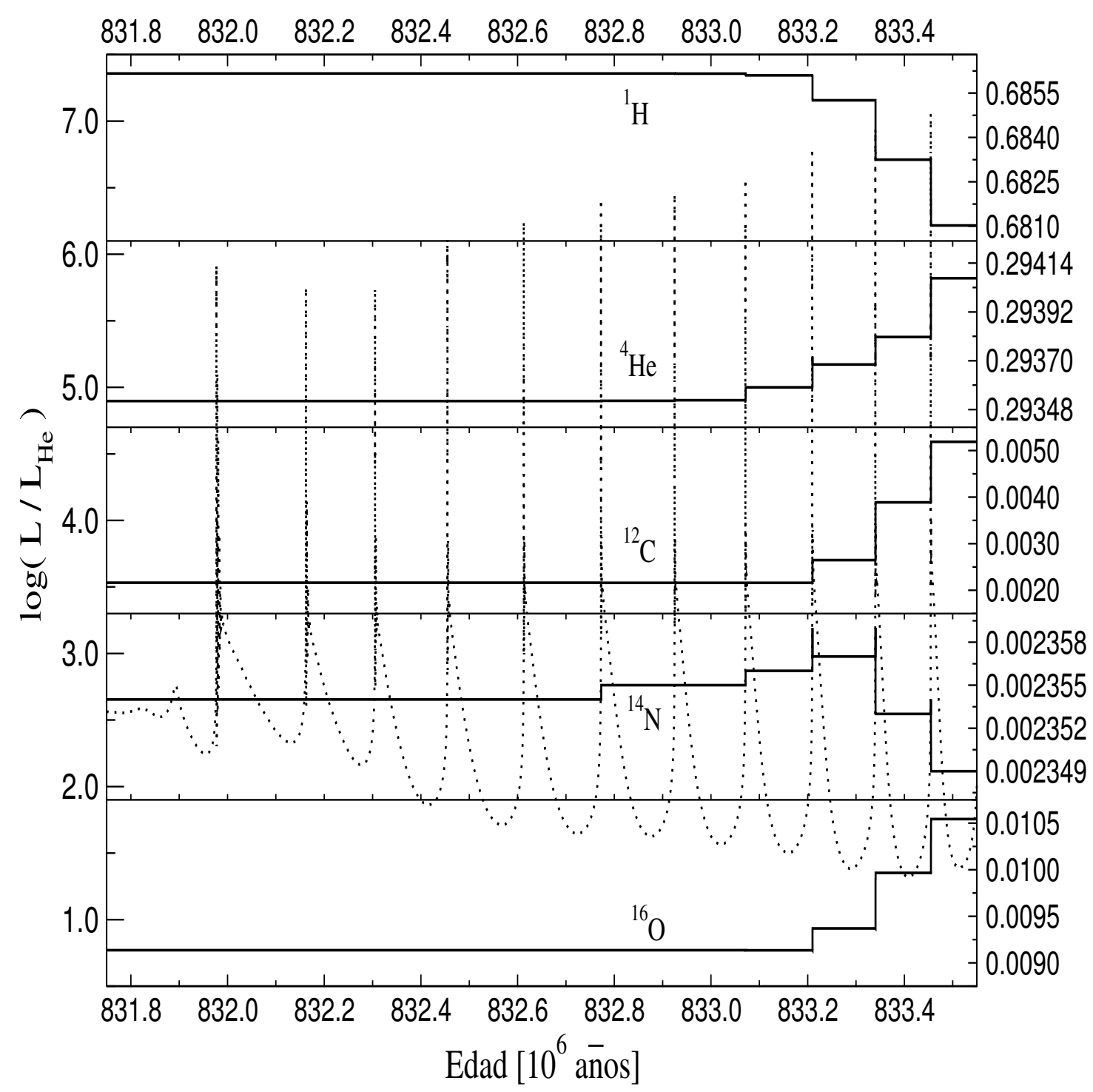

Figura 16: Abundancias superficiales y $3^{\text {er }}$ "dredge up", para una estrella con masa $M_{Z A M S}=$ $2.5 M_{\odot}$. Se han agregado los pulsos debido a la quema de helio. Observar como a partir del $8^{v o}$ pulso se produce un enriquecimiento en la superficie estelar con helio, carbono y oxígeno, y una disminución del hidrógeno. 


\section{$5.2 \quad$ El rol del overshooting}

La formación de las estrellas de carbono y su modelado mediante los cálculos de evolución estelar ha sido un problema desde hace mucho tiempo. Los primeros cálculos de evolución estelar predijeron el MCE, en su mayoría, para estrellas con un núcleo masivo, esto es a altas luminosidades, mientras que la mayoría de las estrellas de carbono observadas se encuentran a bajas luminosidades y, por lo tanto, son de baja masa (Iben, 1981). Además, varios cálculos evolutivos no encontraron MCE para todas o difieren en la eficiencia obtenida [por ejemplo (Vassiliadis and Wood, 1993b); (Blöcker, 1995b); (Forestini and Charbonnel, 1997)]. La falta de estrellas de carbono ópticamente brillantes puede ser explicada en términos del HBB y de la pérdida de masa. El HBB contrarrestra la formación de estrellas de carbono. Sin embargo, puesto que él cesa antes que el MCE los episodios finales de MCE pueden todavía producir estrellas de carbono (Frost et al., 1998). Debido a la pérdida de masa se esperaría que tales estrellas luminosas de carbono estén ocultas por el polvo escondiéndolas de las mediciones ópticas. En efecto, las observaciones infrarrojas de van Loon (van Loon et al., 1999) muestran evidencia para la existencia de estrellas de carbono luminosas oscurecidas. La falla para obtener un eficiente MCE, en particular para modelos AGB de baja masa, está relacionada a la descripción aproximada de la convección estelar y de acuerdo al tratamiento de los límites convectivos. Otra cuestión que está íntimamente ligada a este problema es cómo producir una suficiente cantidad de ${ }^{13} \mathrm{C}$ en el interior como la fuente de neutrones para la nucleosíntesis de procesos $s$. Por lo tanto, frecuentemente se concluye que la mezcla puede tomar lugar fuera de los límites convectivos [(Iben, 1976); (D’Antona and Mazzitelli, 1996); (Wood, 1997)].

Para sobrellevar estos problemas Herwig y colaboradores (Herwig et al., 1997) han considerado overshooting (OV) difusivo para todos los límites convectivos durante toda la evolución conduciendo a considerables cambios en los modelos. El OV empleado está basado sobre los cálculos hidrodinámicos de Freytag (Freytag et al., 1996) quien mostró que la mezcla toma lugar más allá del borde clásico de Schwarzschild debido al OV con un campo de velocidad que declina exponencialmente. En la región del OV el coeficiente de difusión correspondiente está dado por $D_{o s}=v_{0} \cdot H_{p} \cdot \exp \frac{-2 \cdot \zeta}{f \cdot H_{p}} \operatorname{con} v_{0}$ : velocidad de los elementos convectivos inmediatamente antes del borde de Schwarzschild; $\zeta$ : distancia desde el borde de la zona convectiva; $f$ : parámetro de eficiencia del OV. En nuestros cálculos de evolución estelar el parámetro de eficiencia fue elegido como $f=0.015$. Este método provee para las estrellas AGB una cantidad suficiente de MCE para formar estrellas de carbono de baja masa como las de las observaciones. Esto conduce a la formación de ${ }^{13} \mathrm{C}$ como fuente de neutrones para producir los procesos $s$ en estas estrellas -ver también (Herwig et al., 1999b).

Por un lado, estos cálculos muestran que el MCE puede obtenerse fácilmente, si existe 
alguna envoltura con OV para superar la discontinuidad del $\mathrm{H} / \mathrm{He}$ mediante el hundimiento de la envoltura convectiva. Por otro lado, el OV conduce también a un aumento de la zona convectiva conductora de los pulsos y a incrementar la mezcla del material nuclear desde las capas profundas pertenecientes a la capa de helio hacia la zona intercapa ("dragado de la intercapa", esta zona está ubicada por encima de la zona convectiva conductora de los pulsos y por debajo de la envoltura convectiva) resultando así un considerable cambio de las abundancias. Después del dragado de la intercapa las abundancias (en fracción de masa) de $(\mathrm{He}, \mathrm{C}, \mathrm{O})$ son típicamente iguales a $(0.40,0.40,0.16)$ en lugar de $(0.70,0.25,0.20)$ como en secuencias sin OV (Blöcker, 2001). Es importante notar que la cantidad total de dragado depende principalmente de la intensidad del dragado de la intercapa anterior (Herwig et al., 1999b). Estas abundancias modificadas de la intercapa son cercanas a las abundancias superficiales observadas de las estrellas centrales Wolf-Rayet y a las de las estrellas PG1159.

\subsection{Hot Bottom Burning (HBB)}

En las estrellas AGB más masivas $\left(M_{Z A M S} \gtrsim 4 M_{\odot}\right)$, la envoltura se extiende tan hacia adentro que puede penetrar dentro de la capa de quema de hidrógeno durante la fase entre pulsos, este fenómeno es conocido como Hot Bottom Burning (HBB) o "quema de la envoltura" -(Iben, 1975); (Scalo et al., 1975).

La consideración de overshooting difusivo para todos los límites convectivos provee a las estrellas AGB una cantidad suficiente de dredge up como para formar estrellas de carbono de baja masa como lo requieren las observaciones. Esto conduce a la formación de ${ }^{13} \mathrm{C}$ como una fuente de neutrones para producir el proceso $s$ en estas estrellas. El HBB, es decir la penetración de la envoltura convectiva dentro de la capa de quema hidrógeno, provee de estrellas AGB ricas en litio y puede retrasar o evitar el estado de estrella de carbono mediante el cambio de ${ }^{12} \mathrm{C}$ en ${ }^{13} \mathrm{C}$ y ${ }^{14} \mathrm{~N}$.

En la parte superior de la AGB, la quema de helio en capa se vuelve inestable aumentando los pulsos térmicos. Durante estas inestabilidades la luminosidad de la capa de helio aumenta rápidamente por un tiempo corto, de unos 100 años de $10^{6} L_{\odot}$ a $10^{8} L_{\odot}$. Esta enorme cantidad de energía producida fuerza al desarrollo de una zona convectiva conductora de los pulsos la cual mezcla los productos de la quema de helio, carbono y oxígeno, dentro de la región de la intercapa. Puesto que la capa de hidrógeno es empujada dentro de regiones más frías la quema de hidrógeno cesa temporariamente permitiendo a la envoltura convectiva moverse hacia abajo después del pulso, para penetrar en aquellas regiones de la intercapa enriquecidas anteriormente con carbono y mezclar ese material hacia la superficie ( $3^{\text {er }}$ dredge $u p$ ). El overshoot conduce a un alargamiento de la zona convectiva conductora de los pulsos y a aumentar la mezcla del material del núcleo desde las capas profundas debajo de la capa 
de helio a la zona de la intercapa (dredge up de la intercapa) y a ahondar la convección de la envoltura. Debido al overshoot se encuentran temperaturas elevadas en la base de la zona convectiva conductora de los pulsos. En la fig. (17), se representa temperatura vs. edad para estrellas con masas $M_{Z A M S}=3,4,5$ y $6 M_{\odot}$.

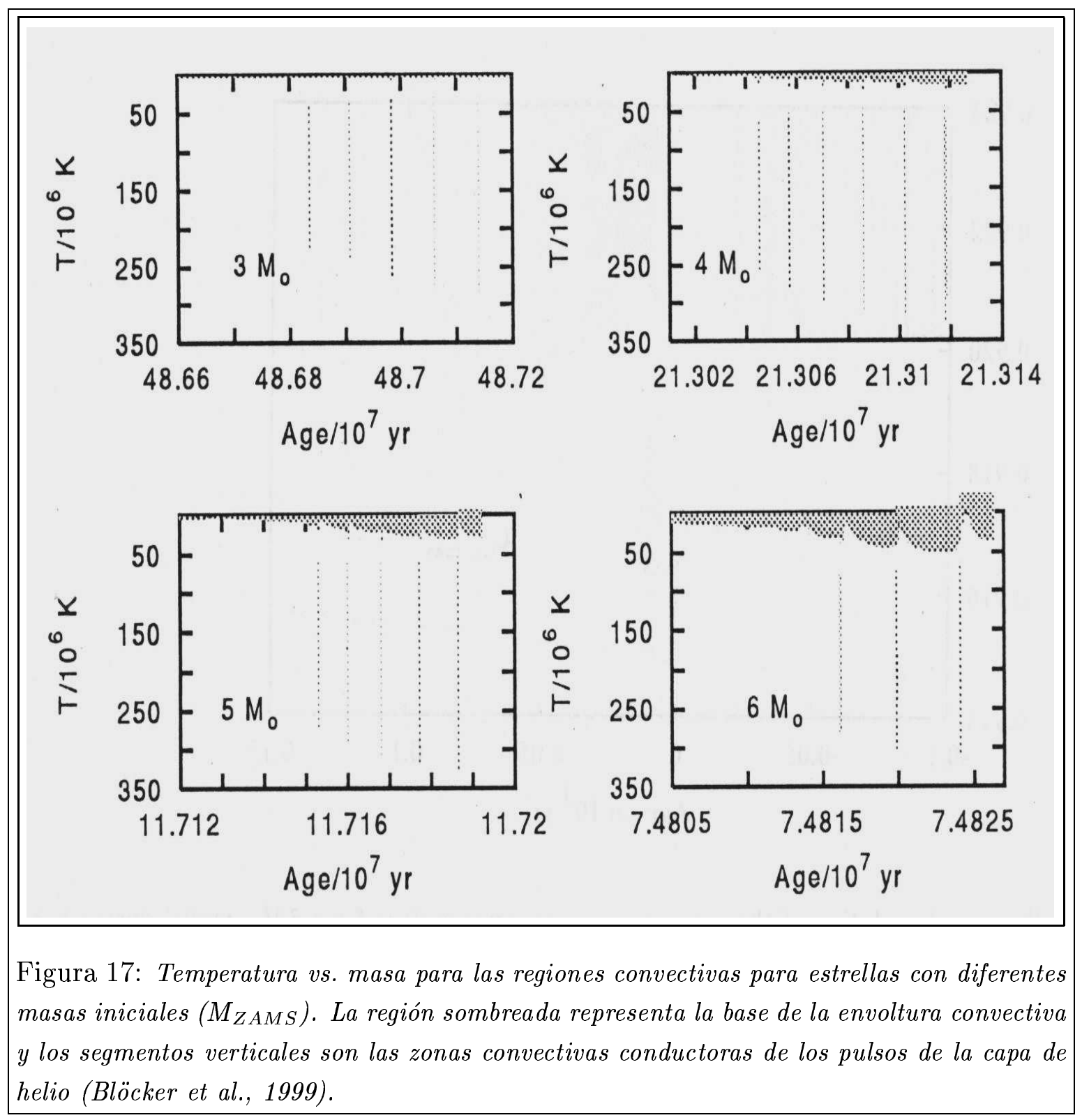

Vemos que para una estrella con $M_{Z A M S}=3 M_{\odot}$ las temperaturas están cerca a los $300 \times 10^{6} \mathrm{~K}$, el umbral de temperatura para que opere la fuente de neutrones ${ }^{22} \mathrm{Ne}(\alpha, n)^{25} \mathrm{Mg}$. Para modelos más masivos se muestran temperaturas de $350 \times 10^{6} \mathrm{~K}$ y más, después de unos pocos pulsos. Así, en estas estrellas, los procesos de nucleosíntesis $s$ estarán governados por las dos reacciones siguientes: ${ }^{13} \mathrm{C}(\alpha, n){ }^{16} \mathrm{O}$ y ${ }^{22} \mathrm{Ne}(\alpha, n){ }^{25} \mathrm{Mg}$, ambas fuentes de neutrones. 
En la base de la envoltura convectiva se alcanzan temperaturas por encima de $50 \times 10^{6} \mathrm{~K}$ y el material que es quemado ahí, inmediatamente es mezclado hacia la superficie (ver fig. 18). Debido al ciclo CNO de la envoltura el ${ }^{12} \mathrm{C}$ puede ser transformado en ${ }^{13} \mathrm{C}$ y ${ }^{14} \mathrm{~N}$. En consecuencia una baja relación ${ }^{12} \mathrm{C} /{ }^{13} \mathrm{C}$ es una señal típica de $\mathrm{HBB}$, el cual puede evitar que estrellas AGB se conviertan en estrellas de carbono -(Iben, 1975); (Boothroyd et al., 1993); (Frost et al., 1998).

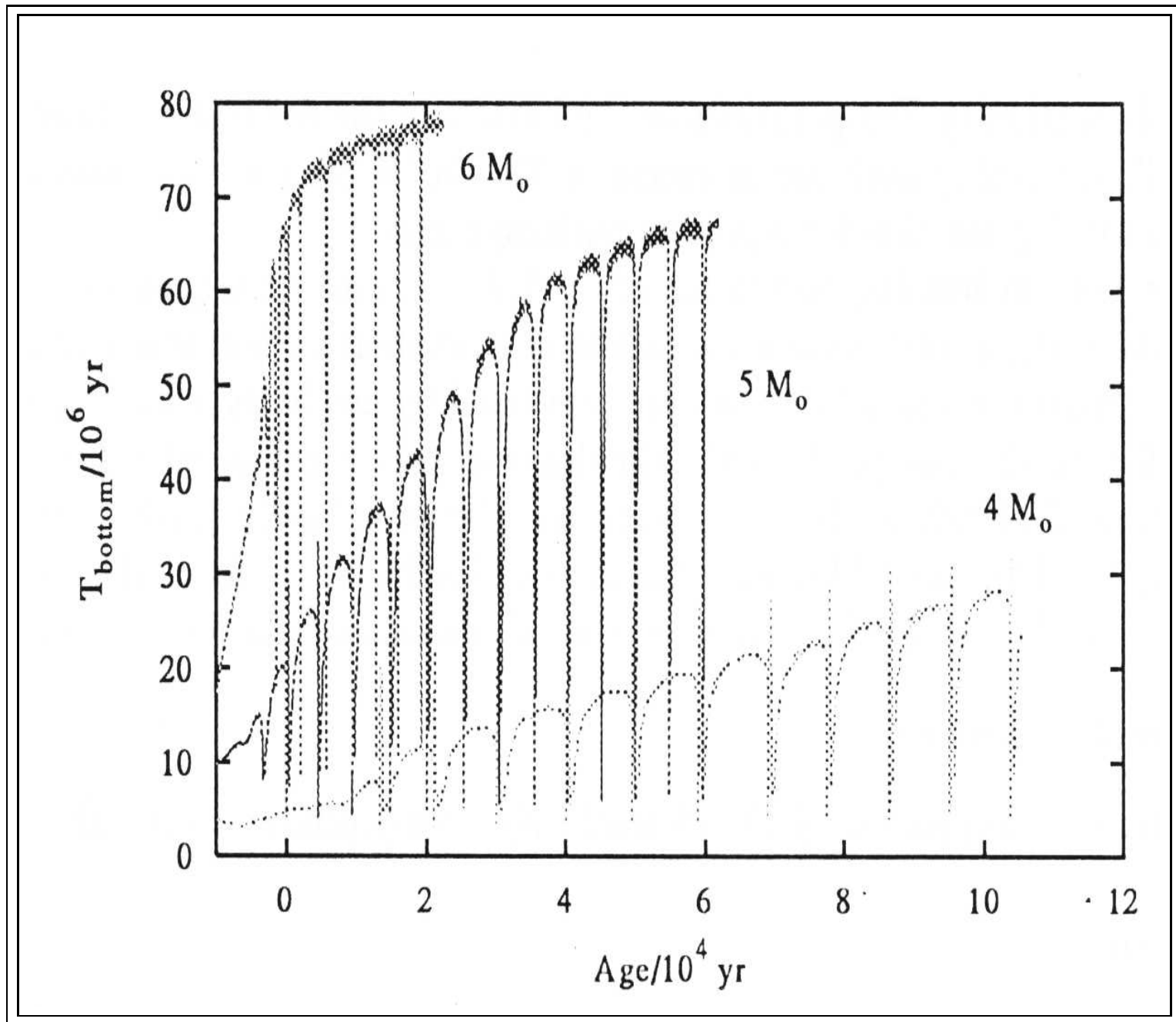

Figura 18: Temperatura de la base de la envoltura convectiva ( $\left.T_{b o t t o m}\right)$ para modelos con masas $M_{Z A M S}=4,5$ y $6 M_{\odot}$. El fuerte aumento de $T_{\text {bottom }}$ indica la eficiencia del HBB. El timepo $t=0$ se refiere al máximo de $L_{H e}$ del primer pulso térmico (Blöcker et al., 1999).

La evolución de las abundancias superficiales depende de la competencia entre HBB y el dredge up. Ambos efectos dependen de la masa de la envoltura, esto es de la pérdida de masa. En consecuencia, la relación C/O alcanzada al final de la evolución AGB depende en forma crucial de qué proceso se apaga primero. La masa crítica mínima de la envoltura para 
un eficiente HBB parece ser mayor que la necesaria para el dredge up (Frost et al., 1998), y para que la estrella de carbono se forme depende de la eficiencia del dredge up remanente. Si se considera overshoot, el dredge up opera aún durante el estado de post-AGB obteniéndose conclusiones importantes para la formación de estrellas deficientes en hidrógeno.

Otra consecuencia directa del HBB es la formación de estrellas ricas en litio (Scalo et al., 1975). Los tiempos de escala de los decaimientos $\beta$ del ${ }^{7} \mathrm{Be}$ el cual es prducido en la base de la envoltura convectiva es comparable a los tiempos de escala convectivos. Por lo tanto, antes de que el ${ }^{7} \mathrm{Be}$ sea quemado en la parte inferior de la envoltura, éste puede ser mezclado hacia arriba a las capas más frías donde decae para producir ${ }^{7} \mathrm{Li}$ el cual, es llevado hacia la superficie por convección. Este mecanismo es efectivo para temperaturas entre 30 y $80 \times 10^{6} \mathrm{~K}$ para la base de la envoltura. La producción de estrellas AGB ricas en litio puede ser posible con un tratamiento simultáneo de mezcla y quema. En cualquier otro caso, el ${ }^{7}$ Be podría ser quemado en la base de la envoltura antes de que éste sea mezclado hacia la superficie.

\subsection{La barrera de entropía}

La barrera de entropía es la encargada de evitar que el borde exterior de la capa convectica alcance la interfase de hidrógeno-helio $(\mathrm{H}-\mathrm{He})$, esa interfase es la región comprendida entre ambas regiones de quema -ver fig. (19). Esta barrera está presente a través de esa interfase durante el pulso - ver fig. (19). La contribuyente dominante a la magnitud de esta barrera es la presión de radiación -ver gráfico de la presión de radiación en fig. (20). Similarmente el proceso de dredge up puede entenderse en términos de muchas tasas diferentes de cambios de entropía a cada lado de la interfase de $\mathrm{H}-\mathrm{He}$.

Durante el largo período que hay entre pulsos, cuando la generación de energía debida a las reacciones de quema de helio cae a valores insignificantes - ver fig. (20)-, la entropía de la materia en la región rica en hidrógeno de la estrella es significativamente mayor que la entropía en la región rica en helio, justo debajo de la interfase $\mathrm{H}-\mathrm{He}$-ver fig. (19). En un intervalo muy angosto a través de la interfase $\mathrm{H}-\mathrm{He}$, la entropía aumenta hacia un valor de alrededor de $5 k / \mu M_{H}$ ( $k$ : constante de Boltzmann, $\mu$ : peso molecular medio, $M_{H}$ : masa atómica del $\mathrm{H}$ ), esto es la altura de la barrera que posee la capa 3. Esto sucede en un intervalo pequeño de tiempo que comprende al pulso de helio. Más de la mitad de este salto de entropía, se debe al rápido aumento en la relación de la presión de radiación a la presión del gas a través de la interfase $\mathrm{H}-\mathrm{He}$.

Mientras sucede el pulso toda la energía nuclear liberada por las reacciones de quema de helio es convertida localmente en energía térmica y en el trabajo de expansión -figs. (19) y (20). Cuando el pulso térmico comienza a disminuir rápidamente, el reservorio de energía atrapado en la región rica en helio es capaz de aumentar $S$ por un valor de $7 k / \mu M_{H}$ para la 


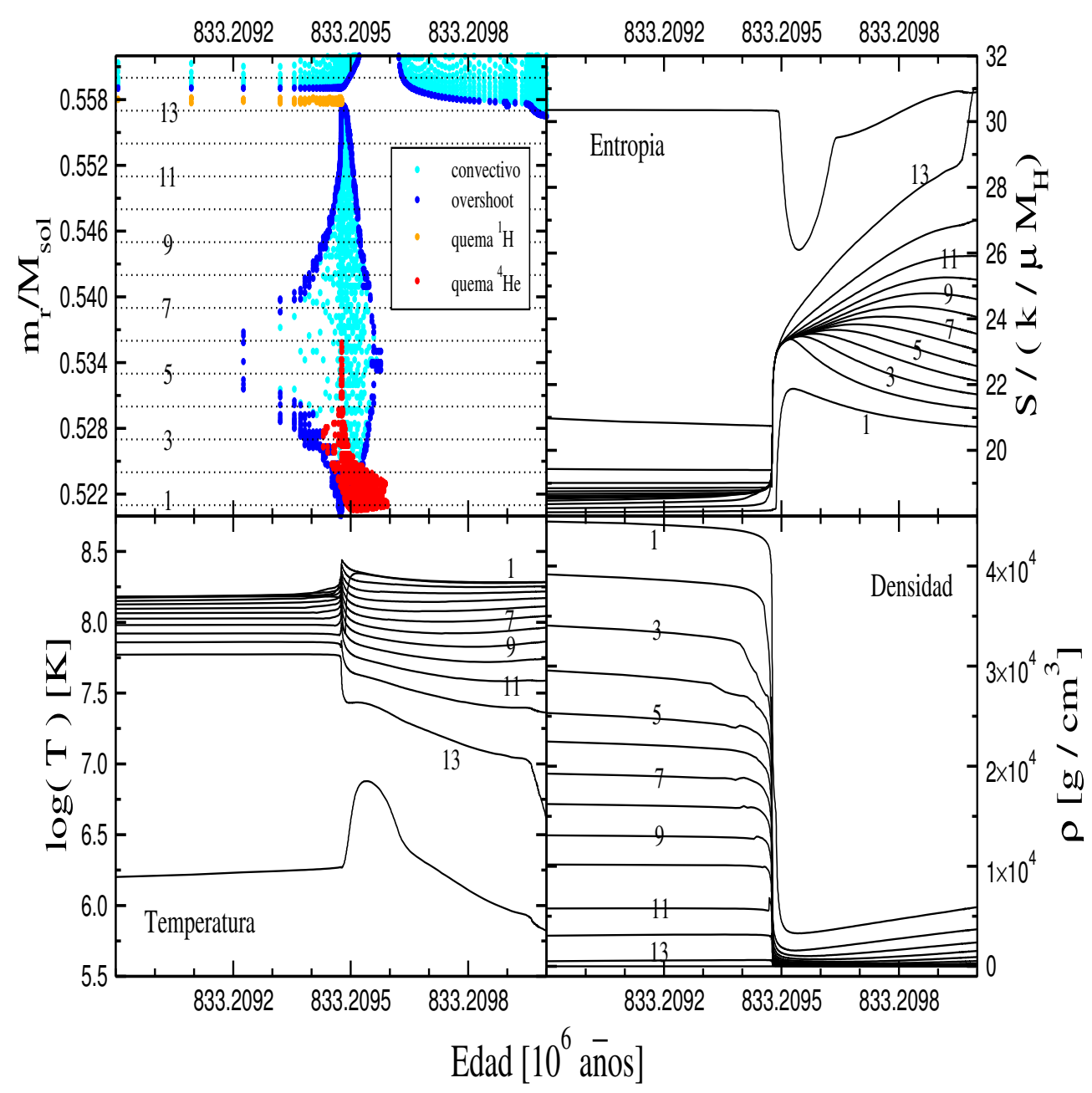

Figura 19: (a) En la figura superior izquierda mostramos, a partir de nuestros cálculos, la región interna de la estrella en donde está presente la capa conductora del gno pulso. Se ha considerado para la quema de hidrógeno y helio que $\varepsilon_{H}>10^{3} \mathrm{erg}^{-1} \mathrm{~s}^{-1}$ y $\varepsilon_{H e}>3 \times 10^{6} \mathrm{erg} \mathrm{g}^{-1} \mathrm{~s}^{-1}$. Los números corresponden a un valor dado para distintas coordenadas masa. (b) En la figura superior derecha se muestra como la barrera de entropía se levanta hacia el máximo de $\varepsilon_{H e}$. (c) En la figura inferior izquierda se grafica la marcha de la temperatura con el tiempo para distintas coordenadas masas, se observa que para la capa 1 la temperatura aumenta en $\simeq 100 \times 10^{6} \mathrm{~K}$ (de 158 a 251 en $\left.10^{6} \mathrm{~K}\right)$. (d) En la figura inferior derecha tenemos densidad vs. tiempo, observar que después del pulso la densidad de la capa 1 , cae $\approx 1$ orden de magnitud (de $\approx 4.4$ a 0.45 en unidades de $10^{4} \mathrm{~g}$ $\left(\mathrm{cm}^{-3}\right)$. 


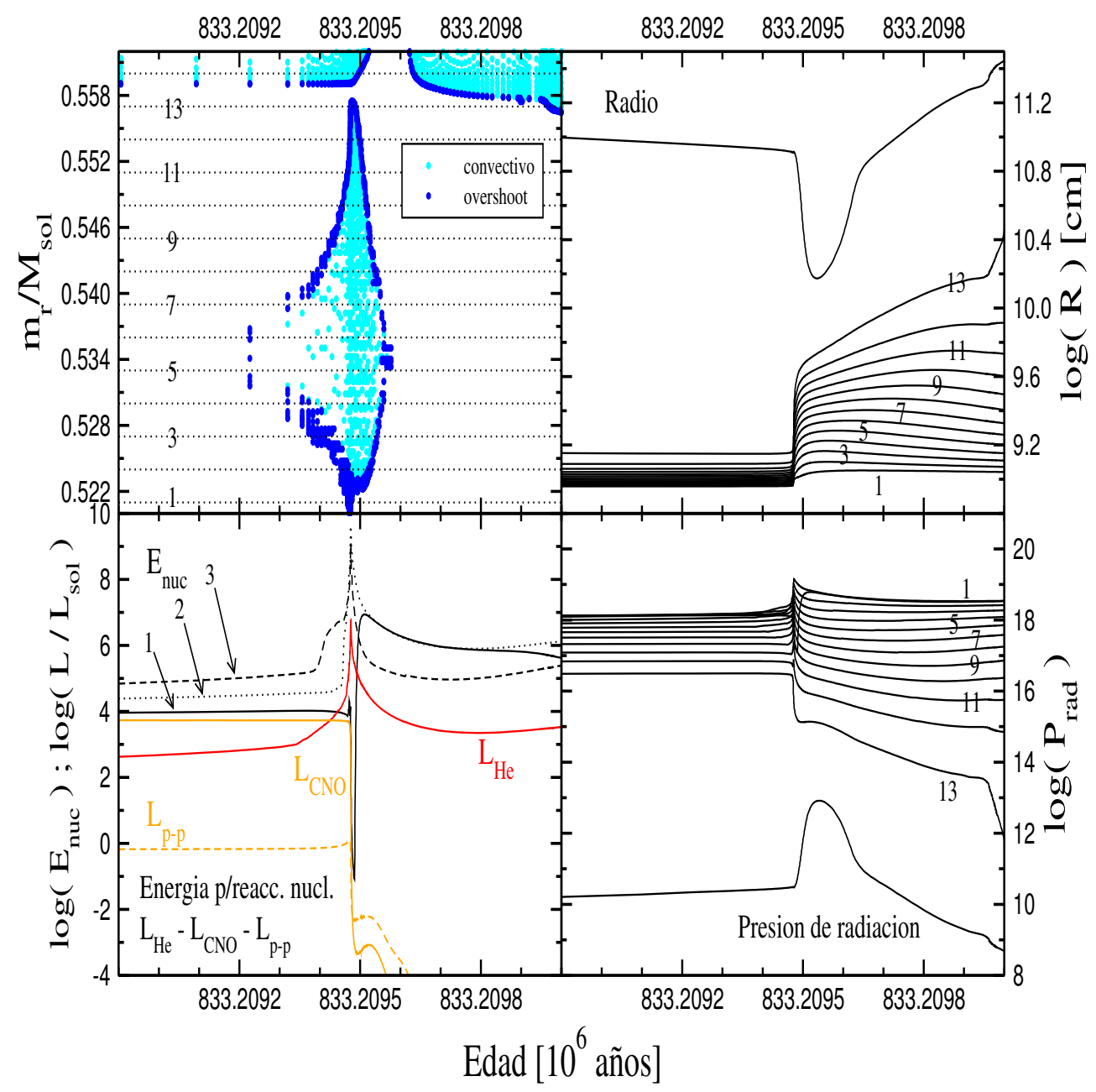

Figura 20: (a) Para la figura superior izquierda ver los comentarios de la fig. (19). (b) En la figura superior derecha se grafica el radio en función del tiempo. Notar cómo se expande la estrella en la parte superior de la capa convectiva (capa 13) moviéndose $\approx 2.37 \times 10^{5} \mathrm{~km}$ hacia afuera $\left(\approx \frac{1}{3} R_{\odot}\right.$, aumenta de 0.141 a $2.511 \times 10^{10} \mathrm{~cm}$ ). (c) En la figura inferior izquierda se representa la liberación de energía nuclear $\left(\varepsilon_{n u c}\right)$ para las capas 1, 2, y 3, estas capas corresponden a la zona en donde $\varepsilon_{n u c}$ es mayor. Se agregan también las luminosidades integradas en toda la estrella $L_{p-p}, L_{C N O}$ y $L_{H e}$. (d) En la figura inferior derecha se grafica la presión de radiación vs. tiempo. 
parte superior de la capa convectiva -ver capa 13 de la fig. (19).

Cuando la capa convectiva está en su máxima extensión la entropía de su parte superior, puede crecer (desde antes del pulso) en un valor de $10 k / \mu M_{H}$ hasta alcanzar la base de la envoltura convectiva -fig. (19)- más allá de la interfase H-He. Esto es, la barrera de entropía se levanta durante la fase del interpulso evitando el contacto entre el borde exterior de la capa convectiva y la envoltura convectiva rica en hidrógeno e impide el contacto con la materia más allá de la interfase $\mathrm{H}-\mathrm{He}$.

El fenómeno del dredge up puede ser atribuido a un rápido aumento de entropía en la región radiativa justo debajo de la base de la envoltura convectiva y a la lentitud, con la cual el gran reservorio de entropía representado por la envoltura, responde a ese aumento.

\subsubsection{La barrera de entropía previene el contacto entre las regiones de la capa y la envoltura convectiva}

En una región en donde el peso molecular es constante y donde la degeneración electrónica puede ser despreciada, la tasa para la cual el calor penetra un gramo de materia para ser convertida en energía térmica y en trabajo puede ser escrita como -ver (Iben, 1976):

$$
\frac{d Q}{d t}=T \frac{d S}{d t}=\frac{k T}{\mu M_{H}} \frac{d \bar{S}}{d t},
$$

donde

$$
\bar{S}=\ln \left(T^{3 / 2} \cdot \frac{\mu}{\varrho}\right)+\frac{4 P_{R}}{P_{G}},
$$

Aquí $T$ y $\varrho$ son la temperatura y la densidad respectivamente, $\mu$ es el peso molecular medio, y $P_{R}$ y $P_{G}$ son la presión de radiación y la presión del gas respectivamente. Si los cambios del peso molecular son tomados en cuenta en $d Q / d t$, entonces $d \bar{S} / d t$ en la ec. (23) debe ser reemplazado por $d \bar{S} / d t-\frac{3}{2} d(\ln \mu) / d t$. En la mayoría de todos los casos, los cambios del peso molecular pueden ser despreciados al calcular $d Q / d t$. Cuando $d \mu / d t$ es grande, entonces $\varepsilon_{\text {nuclear }} \gg d Q / d t$; y cuando $d Q / d t \gg \varepsilon_{\text {nuclear }}$, entonces $d \mu / d t$ es pequeño. La única excepción está cerca de una discontinuidad del peso molecular.

En la fig. (19) se muestra la distribución del parámetro de entropía $\bar{S}$ (o la entropía en unidades de $k / \mu M_{H}$ ) dentro de una región de producción de energía nuclear durante el $9^{n o}$ pulso térmico. El borde exterior de la capa convectiva alcanza su máximo exterior extendido en masa después del pico del pulso y de allí en adelante comienza a retroceder hacia dentro en masa fig. (20). La energía nuclear ha sido convertida en energía térmica por gramo y en trabajo por gramo $\left(k T \Delta \bar{S} / \mu M_{H}\right)$, y una cantidad considerable de trabajo ha sido empleado 
en expandir la materia en la región de quema de helio -ver fig. (20) grafico de $\log R$ vs. tiempo.

La presión de radiación es de principal importancia para establecer la barrera de entropía. Notar que en el momento, en que el borde exterior de la capa convectiva, ha alcanzado su máxima extensión en masa, la temperatura de la interfase $\mathrm{H}-\mathrm{He}$ ha caído un valor $52 \times 10^{6} \mathrm{~K}$ (es decir de $\log T \simeq 7.750$ a 6.625 -de $56 \times 10^{6}$ a $4 \times 10^{6} \mathrm{~K}$ - para la capa 13), esto es la generación de energía por la quema de hidrógeno contribuye muy poco al flujo de energía hacia la superficie. El flujo saliente es mantenido por la liberación de energía térmica y por la liberación de energía a partir de las reacciones de quema de helio.

La separación mínima lograda entre la interfase $\mathrm{H}-\mathrm{He}$ y el borde exterior de la capa convectiva es lo suficientemente pequeña, como para que la posibilidad de contacto entre la capa y la interfase permanezca abierta. Sin embargo la máxima proximidad es lograda después de que el pulso se ha ido hacia su fase declinante, y durante la fase de crecimiento del siguiente pulso.

Puesto que sólo una fracción de la altura de escala de la presión separa a la capa y la interfase para el mínimo de separación, uno debe esperar algo del OV de la materia desde la capa convectiva dentro de la región rica en hidrógeno, y consecuentemente, algo de goteo de hidrógeno en la capa convectiva.

\subsubsection{El MCE y el carbono estelar}

Mientras la capa convectiva decrece en tamaño, la región justo fuera de la interfase de $\mathrm{H}-\mathrm{He}$ se vuelve marginalmente inestable hacia la convección. Esto es, en lugar de ser completamente convectivo, como durante la fase entre pulsos, esta región se rompe en una capa pequeña convectiva alternada con una región radiativa - figs. (13) y (14). Esta conducta cesa una vez que la capa convectiva en la región rica en helio desaparece y la capa que quema hidrógeno se extingue completamente. De ahí en más, hay sólo una región completamente convectiva en la estrella, y ella se extiende desde la interfase $\mathrm{H}-\mathrm{He}$ hasta la superficie estelar. Después del pulso, la base de esta única región convectiva comienza a extenderse hacia adentro en masa, dragando primero los productos de una quema completa de hidrógeno y luego los productos de una incompleta quema de helio. La conducta de los límites de la zona convectiva durante el $9^{n o}, 10^{m o}$ y $11^{m o}$ pulsos se muestra en la fig. (14). En cada caso la fase del dragado dura aproximadamente 150 años y lleva hacia adentro de la envoltura una fracción significativa de materia que ha sido procesada por las reacciones de quema de helio en la capa convectiva durante el pico del pulso. El dragado dura el tiempo en que la envoltura desciende en masa, que en la fig. (14) está dada por la inclinación que toma ésta hacia adentro - ver $11^{m o}$ pulso. 


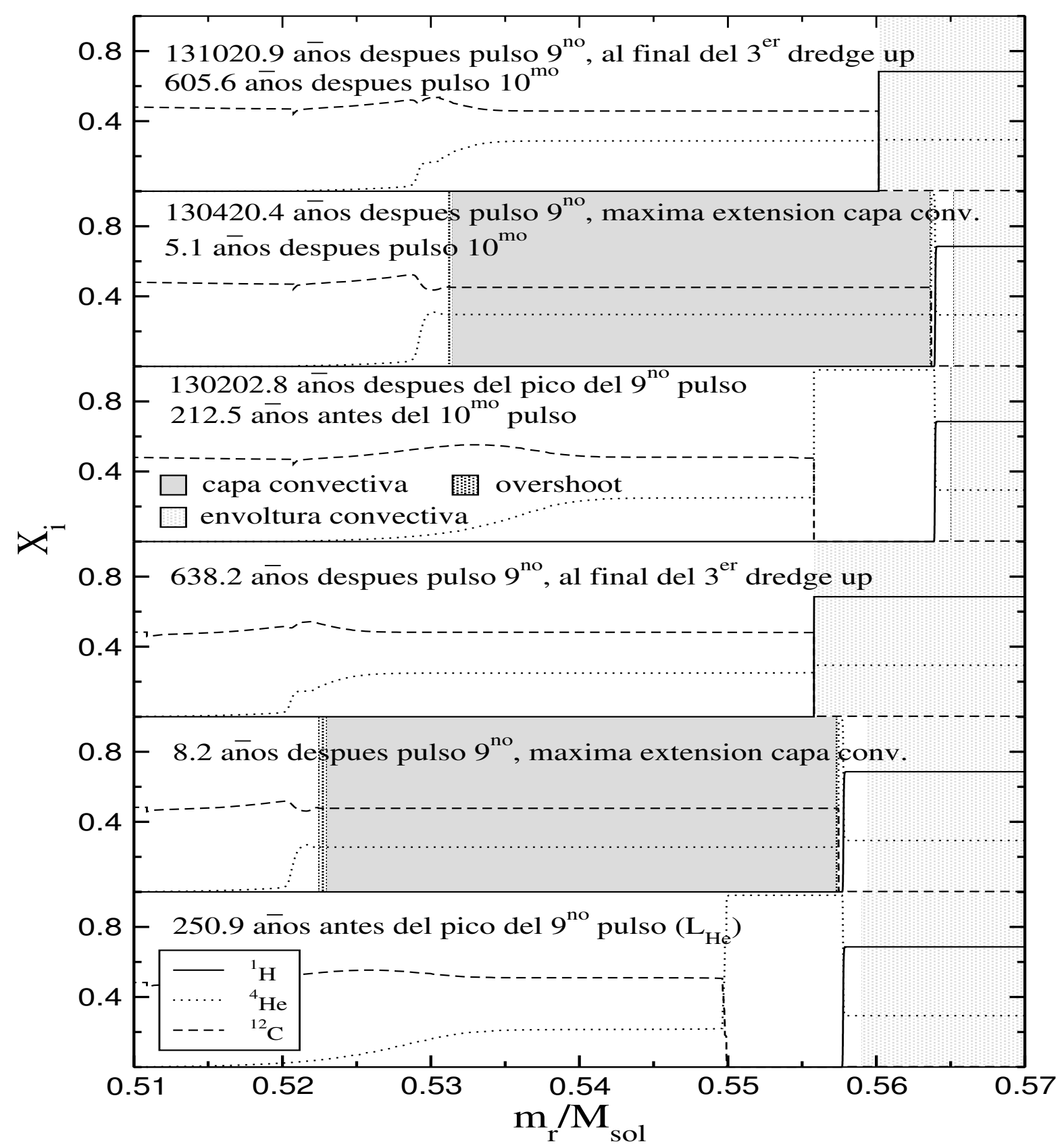

Figura 21: Se grafica el comportamiento del interior de la estrella en una región próxima a la capa convectiva y para varias etapas evolutivas en un entorno del $9^{\text {no }}$ y $10^{\text {mo }}$ pulso. Se muestran los perfiles internos de ${ }^{1} \mathrm{H},{ }^{4} \mathrm{He} y{ }^{12} \mathrm{C}$, las regiones con overshooting y convectivas pertenecientes a la envoltura y a la capa conductora de cada pulso. 
En la fig. (16) vemos el resultado superficial del dragado del ${ }^{12} \mathrm{C}$ hacia la superficie. En la fig. (21) se muestran para varias etapas evolutivas, alrededor de los pulsos $9^{\text {no }}$ y $10^{m o}$, las abundancias superficiales de ${ }^{1} \mathrm{H},{ }^{4} \mathrm{He}$ y ${ }^{12} \mathrm{C}$, el overshooting y la regiones convectivas correspondientes a la envoltura y la capa conductora del pulso. Notar que, para un dado pulso, aproximadamente $\frac{3}{4}$ del ${ }^{12} \mathrm{C}$ que reside en la capa convectiva cuando está en su máxima extensión, es el mismo que ha sido producido durante el pulso previo -ver por ejemplo fig. (21) los dos gráficos inferiores. El $\frac{1}{4}$ restante es producido en la capa convectiva durante el pulso. La abundancia por masa del ${ }^{12} \mathrm{C}$ en la materia que es dragada hacia la envoltura es de $\sim 0.0005$, para el $9^{n o}$ pulso y de $\sim 0.001$ para el $10^{m o}$ y $11^{m o}$-ver fig. (14). La interfase de $\mathrm{H}-\mathrm{He}$ en todos los gráficos de la fig. (21), está ubicada justo donde se produce el escalón del ${ }^{1} \mathrm{H}$.

Puede demostrarse que la temperatura de la base de la envoltura convectiva, es una función de la masa del núcleo de carbono-oxígeno. En ese lugar, ella crece con el aumento de la masa del núcleo y además sucede que, para una masa nuclear lo suficientemente grande, el ${ }^{12} \mathrm{C}$ de la envoltura será destruido más rápido de que éste sea dragado.

\subsubsection{El por qué del MCE y la entropía}

En la fig. (19) se observa que a medida que la capa convectiva retrocede en masa, la entropía de la región radiativa, entre el borde exterior de la capa convectiva y la base de la envoltura convectiva, crece (esto es entre la capa 13 y 14 -esta última no está rotulada). Una vez que la capa convectiva ha desaparecido, ocurre un reajuste en la entropía, la cual está siendo transferida desde la región de producción de energía por las reacciones de quema de helio a la materia sobre el lado radiativo de la interfase de $\mathrm{H}-\mathrm{He}$.

La entropía contenida en la envoltura convectiva por encima de la interfase $\mathrm{H}-\mathrm{He}$ es mayor respecto a la entropía contenida en la región rica en helio debajo de la interfase. Por lo tanto, la absorción de cantidades comparables de energía por cada región, corresponde a un mucho menor aumento en la entropía en la región convectiva por encima de la interfase, que en la región radiativa por debajo de la interfase (capas 13 y 14). La única manera en la cual la continuidad del parámetro de entropía $\bar{S}$ puede mantenerse a través de la interfase es por medio del movimiento de la interfase hacia adentro en masa.

Ya que el MCE es una consecuencia de un ajuste de entropía en la región rica en helio durante la fase de baja potencia de un pulso térmico, esto podría no pensarse como una extensión hacia adentro de la convección de la envoltura. En efecto, la materia rica en carbono es inyectada dentro de una región convectiva de la envoltura a través de una región convectiva intermedia siendo el pulso térmico el único responsable. 


\section{Evolución más allá de la AGB}

Para una estrella con $M_{Z A M S}=2.7 M_{\odot}$, la pérdida de masa termina la evolución AGB, cuando la masa de la envoltura es reducida $a \approx 10^{-2} M_{\odot}$. Entonces la estrella se mueve desde la AGB (Schönberner, 1979) evolucionando hacia el régimen de estrellas centrales de nebulosas planetarias. Análisis sobre la estructura y evolución de estrellas centrales de nebulosas planetarias están dadas por ejemplo por Iben (Iben, 1995), y Schönberner y Blöcker (Schönberner and Blöcker, 1996).

\subsection{Pérdida de masa}

Un aspecto importante de la transformación de estrellas AGB en enanas blancas es el tratamiento de pérdida de masa sobre la AGB y post-AGB. Además, la historia de pérdida de masa en la AGB determina la estructura interna de la estrella alcanzada en el pico de la AGB y por lo tanto la disminución de la velocidad a lo largo de la parte de enfriamiento de la evolución post-AGB (Blöcker, 1995b; Blöcker, 1995a). Por otro lado, los tiempos de transición desde la AGB a la región de estrella central depende sensiblemente de las tasas de pérdida de masa empleadas más allá de la AGB (Schönberner, 1983). Las observaciones indican que la pérdida de masa podría decrecer por órdenes de magnitud durante esta fase de transición (Perinotto, 1989). Sin embargo, en la actualidad no es bien conocido cómo y para qué rango de temperaturas toma lugar este decrecimiento fuerte. El tiempo de transición podría no ser demasiado largo puesto que las estrellas post-AGB más frías conocidas tienen temperaturas efectivas de alrededor de 5000K (Schönberner and Blöcker, 1993), y las edades cinemáticas de planetarias más jóvenes son sólo del orden de 1000 años.

En la región de nebulosa planetaria la pérdida de masa puede ser descripta por la teoría de viento conducido por radiación (Pauldrach et al., 1988). Las tasas respectivas de pérdida de masa pueden ser adaptadas como $\dot{M}=1.3 \cdot 10^{-15} L^{1.9}$ (Blöcker, 1995a), llevando a tasas de $10^{-8}$ a $10^{-7} M_{\odot} /$ año para remanentes de 0.6 a $0.8 M_{\odot}$. La correspondiente influencia de la velocidad evolutiva depende de la razón relativa entre el ritmo de quema y la pérdida de masa y es únicamente importante para remanentes masivos. Para los objetos que queman hidrógeno el tiempo de cruce total de la estrella luminosa central está por lo tanto unívocamente dado por el posible combustible (esto es la masa de la envoltura) dividida por la luminosidad de hidrógeno. Puesto que la masa de la envoltura decrece y la luminosidad aumenta con la masa del remanente, uno obtiene tiempos de cruce típicos (entre los $10000 \mathrm{~K}$ y hasta el punto de retorno de la trayectoria en el azul, en el diagrama H-R) de $\sim 100000$ años para $0.55 M_{\odot}, 4000$ años para $0.6 M_{\odot}$, y 50 años para $0.94 M_{\odot}$. 


\subsection{Pulsos térmicos finales}

La evolución al salir de la AGB y post-AGB depende de la fase del pulso térmico. La misma se calcula como el cociente entre, la fracción del intervalo de tiempo transcurrido desde el último pulso, y el intervalo de tiempo ocurrido entre los dos pulsos últimos. Se la denomina como $\phi$ y es la fase con la cual la estrella sale de la AGB -ver fig. (26). La evolución post-AGB, según Blöcker (Blöcker, 2001), está dominada por la quema de helio para $0 \leq \phi \leq 0.15$. Para $0.15 \leq \phi \leq 0.3$ ambas capas, que son fuente de energía nuclear, contribuyen con iguales fracciones de luminosidad, para $0.3 \leq \phi \leq 1.0$ la quema de hidrógeno determina la producción de energía nuclear (Iben, 1984). Si la fase cíclica del pulso térmico es suficientemente grande, puede ocurrir un último pulso térmico durante la evolución postAGB transformando un modelo que quema hidrógeno, en uno que quema helio. El flash fuerza a la estrella a expandirse rápidamente hacia las dimensiones de gigante roja, y el remanente evoluciona rápidamente hacia la AGB (escenario de 'renacido' - 'born again'-, aunque ajusta mejor el término 'rejuvenecimiento' que es lo que sufre en realidad la estrella en su aspecto superficial ya que se vuelve gigante roja). Ahí, comienza su evolución postAGB nuevamente, pero ahora como un objeto que quema helio (Iben, 1984). La escala de tiempo de cruce ahora es aproximadamente tres veces mayor que en el caso de objetos que queman hidrógeno. En principio podemos distinguir tres escenarios relevantes de pulsos térmicos finales para las estrellas Wolf-Rayet CSPNe (Blöcker, 2001):

1. Un pulso térmico 'final' de la AGB (AFTP: AGB Final Thermal Pulse), ocurre inmediatamente antes de que la estrella sale de la AGB. En este caso la masa de la envoltura es muy pequeña $\left(\sim 10^{-2} M_{\odot}\right)$. Si el dredge up es capaz de operar aún para esas masas pequeñas de la envoltura, una fracción significativa de la región intercapa será mezclada con la diminuta envoltura conduciendo a la dilusión de hidrógeno y el enriquecimiento con el carbono y el oxígeno. Sin embargo, los cálculos evolutivos standard predicen un decrecimiento de la eficiencia del MCE para envolturas de baja masa (Wood, 1981) y la falta de una suficientemente alta abundancia de oxígeno en la región de la intercapa.

2. Un pulso térmico 'tardío' (LTP: Late Thermal Pulse), ocurre cuando el modelo evoluciona con aproximadamente luminosidad constante desde la AGB hacia el dominio de las enanas blancas. Esta clase de pulso térmico es similar a aquellos experimentados por las estrellas AGB, pero la masa de la envoltura es aún más pequeña que para el AFTP $\left(\sim 10^{-4} M_{\odot}\right)$. Los cálculos evolutivos sin OV [por ejemplo (Blöcker and Schönberner, 1997)] predicen sólo una suave mezcla, si la hay. La envoltura convectiva no alcanza las capas enriquecidas con carbono, y no ocurre ningún $3^{\mathrm{er}} \mathrm{MCE}$. Por lo tanto, este escenario frecuentemente ha sido considerado en conección con la pérdida 
de masa, el cual, sin embargo, no puede exponer capas libres de hidrógeno antes de que sean alcanzadas temperaturas efectivas de $100000 \mathrm{~K}$ [(Iben, 1984); (Schönberner and Blöcker, 1992)]. Más adelante veremos que es necesario OV para que pueda diluir o quemar el hidrógeno.

3. Un pulso térmico 'muy tardío' (VLTP: Very Late Thermal Pulse), ocurre cuando el modelo está ya sobre la rama de enfriamiento de enana blanca, esto es, después del cese de la quema de hidrógeno. En este caso la zona convectiva conductora del pulso de la capa que quema el helio alcanza y penetra a la capa de hidrógeno causando una considerable o aún una quema total del hidrógeno [(Fujimoto, 1977); (Schönberner, 1979); (Iben, 1984); (Iben and MacDonald, 1995)]. Debido a la mezcla de la muy diminuta envoltura $\left(\lesssim 10^{-4} M_{\odot}\right)$ con fracciones significativas de 100 veces más masiva región de la intercapa, las abundancias resultantes superficiales de carbono y oxígeno están cerca a aquellas de la región de la intercapa. Este escenario ha sido considerado como el más prometedor para formar estrellas Wolf-Rayet CSPNe aunque falla en el ajuste de las abundancias observadas de oxígeno.

En síntesis, uno puede concluir que dentro de los cálculos evolutivos standard ni el AFTP ni el LTP o VLTP parecen ser escenarios bien satisfechos para estrellas Wolf-Rayet, dejando todavía estas estrellas como enigmáticas. Sin embargo, la consideración del OV conduce a un cambio considerable en los modelos haciendo estos escenarios mucho más prometedores.

\subsection{Pulso térmico final de la AGB (AFTP)}

En este escenario la fase es $\phi \approx 0$. Si el OV es aplicado a todas las regiones convectivas, los modelos AGB muestran eficiente dredge up, aún para envolturas de muy baja masa. Entonces, un AFTP puede conducir a ambos, un considerable enriquecimiento con carbono y oxígeno, y a la dilusión del hidrógeno. Las abundancias superficiales resultantes dependen de la masa actual de la envoltura para la cual ocurre el AFTP. Por ejemplo, Herwig (Herwig, 2001a) encuentra que para masas de la envoltura $M_{e n v}=4 \cdot 10^{-3} M_{\odot}$ las abundancias son $(H, H e, C, O)=(0.17,0.33,0.32,0.15)$ después del AFTP. Para obtener una probabilidad lo suficientemente alta para que ocurra el AFTP para masas muy pequeñas de la envoltura (esto es, para $\phi \approx 0$ ) requiere, sin embargo, un acoplamiento de la pérdida de masa al ciclo del pulso térmico. Por otro lado, la abundancia de hidrógeno será relativamente alta. Este escenario predice edades cinemáticas pequeñas para la nebulosa planetaria o estrellas centrales WR las cuales emergen directamente de la AGB. En la fig. (22) se observan las trayectorias evolutivas para tres modelos estelares con $\left(M_{Z A M S} / M_{\odot}, M_{W D} / M_{\odot}\right)=(3,0.605)$, 
$(3,0.625)$ y $(5,0.836)$. Los modelos están quemando hidrógeno hasta la extinsión de la capa fuente de energía (Blöcker, 1995a).

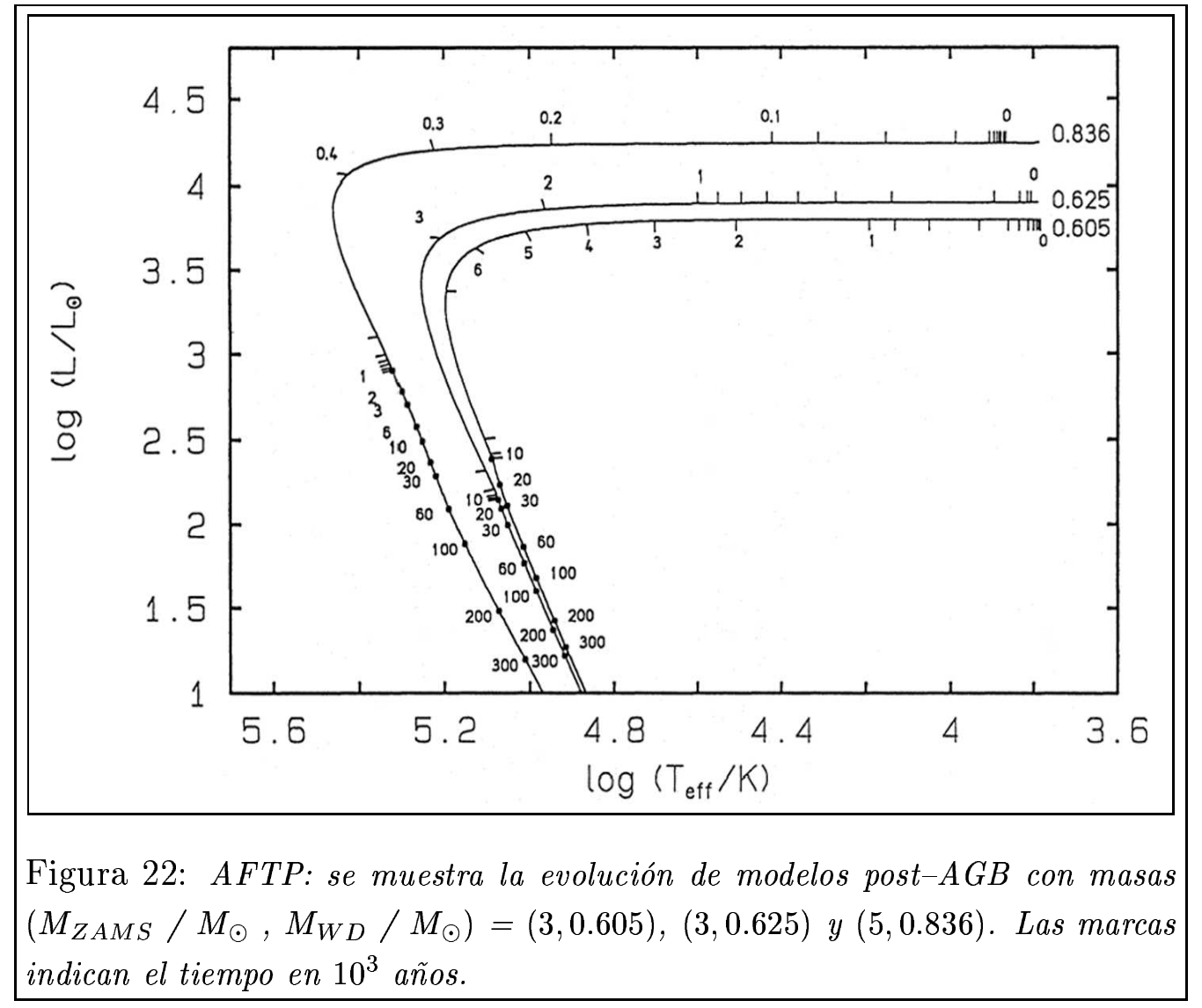

\subsection{Pulso térmico tardío (LTP)}

El LTP, ocurre para aquellas estrellas que abandonan la AGB con $\phi \gtrsim 0.80$ (según Blöcker), sólo han sido consideradas en conección con la pérdida de masa para explicar las abundancias superficiales exóticas de WR CSPNe puesto que el MCE está ausente. En contraste con los objetos que queman hidrógeno donde la gran pérdida de masa conduce a una fuerte aceleración de la evolución limitando la masa removible, las escalas grandes de tiempo evolutivo de los objetos que queman helio permiten exponer las capas libres de hidrógeno, aunque sólo para $T_{\text {eff }}$ por encima de $100000 \mathrm{~K}$. Se requieren fuertes vientos estelares para quitarse suficiente masa para exponer las capas profundas las cuales ajustan a las abundancias de carbono y oxígeno. Aunque este escenario parece no ser aplicable a las WR CSPNe, puede tener alguna relevancia para la más exótica de las estrellas PG1159, la H1504. La H1504 puebla la rama de enfriamiento y muestra una fotósfera conteniendo sólo carbono y oxígeno con $50 \%$ de cada uno. Un estudio de Schönberner y Blöcker (Schönber- 
ner and Blöcker, 1992) muestra que los parámetros fotosféricos $\left(T_{e f f}, \log g\right.$, abundancias superficiales) de $\mathrm{H} 1504$ pueden ser ajustados con un modelo LTP de $0.84 M_{\odot}$ sufriendo una tasa de pérdida de masa constante de $10^{-7} M_{\odot} /$ año hasta que es alcanzada la posición de H1054 en el diagrama H-R. Una cuestión sin resolver es si tales tasas de pérdida de masa pueden ser mantenidas sobre la parte de baja luminosidad de la rama de enfriamiento. Sin embargo, estudios de Werner (Werner et al., 1995) dan evidencia de que las enanas blancas calientes pueden sufrir fuertes vientos estelares. La trayectoria evolutiva para este escenario la podemos ver en la fig. (23) (Blöcker, 1995a).

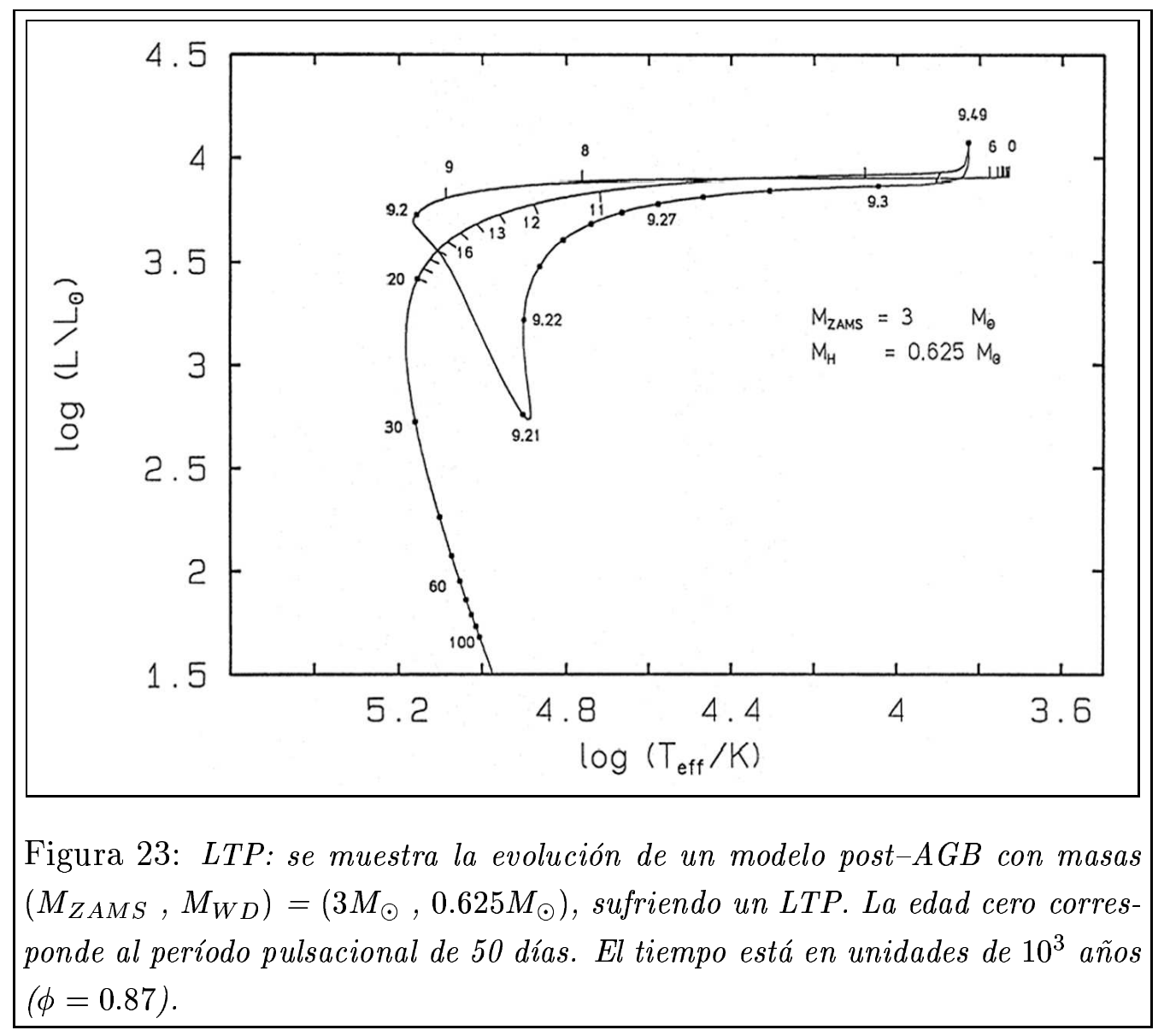

Hasta aquí, los cálculos no mostraron cualquier dredge up mezclando carbono hacia la superficie dentro del escenario LTP. Sin embargo, si es considerado el OV, un eficiente MCE opera aún para $M_{e n v} \lesssim 10^{-4} M_{\odot}$. El escenario LTP puede servir como un canal posible para las WR CSPNe. Notemos que como en el caso de la AFTP, el OV aplicado a la zona convectiva conductora del pulso es esencial para la eficiencia del MCE resultante. Las abundancias de carbono y oxígeno de la intercapa primero aumentan y luego se nivelan después de los pulsos térmicos para modelos de AGB con OV. El OV es necesario para poder explicar estrellas deficientes en hidrógeno producidas a partir de estos dos últimos 
escenarios (AFTP, LTP).

Después del flash el modelo evoluciona hacia el dominio de la AGB. Para el mínimo de temperatura efectiva $(\approx 6700 \mathrm{~K})$ el MCE crece y continúa hasta que la estrella se ha recalentado a $\approx 12000 \mathrm{~K}$ (Blöcker, 2001). El hidrógeno es diluido al $3 \%$ y las abundancias superficiales de helio, carbono y oxígeno son cercanas a aquellas de la región de la intercapa esto es $(\mathrm{He}, \mathrm{C}, \mathrm{O})=(0.45,0.38,0.12)$. Se obtienen las abundancias superficiales como las observadas en las WR CSPNe. La edad cinemática de la nebulosa planetaria es de unos pocos miles de años.

Este escenario se aplica a FG Sagittae (FG Sge) tan bien que sufrió un LTP unos cien años atrás. La FG Sge pareciera haber alcanzado ya su temperatura efectiva mínima y recalentado ahora nuevamente (Kipper, 1996). La masa puede ser estimada cercana a $0.6 M_{\odot}$ y su superficie permanece rica en hidrógeno durante su evolución hacia de vuelta a la AGB. Sin embargo, recientemente la evidencia está creciendo en que FG Sge se vuelve deficiente en hidrógeno durante su recalentamiento (González et al., 1998). Esto es difícil para explicar sin el escenario LTP standard (sin OV) pero está completamente en acuerdo con las predicciones de los nuevos modelos.

\subsection{Pulso térmico muy tardío (VLTP)}

EL VLTP es diferente del AFTP y LTP puesto que él causa un quemado (y mezcla) de la envoltura requiriendo más cálculos numéricos. Uno de los modelos posibles es el de Iben y MacDonald (Iben and MacDonald, 1995), el cual muestra fuerte deficiencia en hidrógeno después del flash pero también bajas abundancias superficiales de oxígeno como para tener en cuenta para las WR CSPNe. Herwig y colaboradores (Herwig et al., 1999a) presentaron el primer modelo VLTP el cual lleva a cabo un acuerdo general con los modelos de abundancias de las WR CSPNe debido a la consideración del OV. Nuevamente, la existencia de un dredge $u p$ en la intercapa provee abundancias de intercapa cercanas a aquellas observadas en las fotósfera de las WR CSPNe parece ser crucial para ajustar a las observaciones.

El VLTP ocurre sobre la rama de enfriamiento cuando la quema de hidrógeno ya se ha apagado. En esta instancia, la zona convectiva conductora del pulso puede alcanzar y penetrar la envoltura rica en hidrógeno debido a la ausencia de una barrera de entropía (Iben, 1976). Entonces, la mayoría de los protones es "tragada" por la zona convectiva donde se sucede el flash de helio y son quemados por completo. Esta región caliente es rica en carbono y los mismos son capturados mediante la reacción ${ }^{12} \mathrm{C}(p, \gamma){ }^{13} \mathrm{~N}$. Es importante destacar que las escalas de tiempo convectivo y las escalas de tiempo nuclear se vuelven comparables durante esta fase, esto hace que los protones sean quemados durante su trayecto hacia el interior. En acuerdo con esto, un tratamiento simultáneo de mezcla y de quema es esencial para computar 
correctamente esta fase evolutiva. El consumo de protones finalmente aumenta un flash de hidrógeno y la energía liberada por este flash conduce a una separación de la convección en una zona superior que genera energía por la quema de hidrógeno y otra zona inferior que genera energía por la quema de helio. Sin embargo, la zona convectiva superior es de vida corta debido a que el posible hidrógeno de la envoltura, es consumido rápidamente. Este flash hace que la estrella sea forzada a evolucionar rápidamente de vuelta hacia la AGB y finalmente convertirse en una estrella central de una nebulosa planetaria a altas temperaturas efectivas pero que ahora es un objeto deficiente en hidrógeno que quema helio en forma quieta. Lo que se espera, es que ese objeto exhiba capas superficiales enriquecidas con elementos producto de la quema de helio, como el carbono.

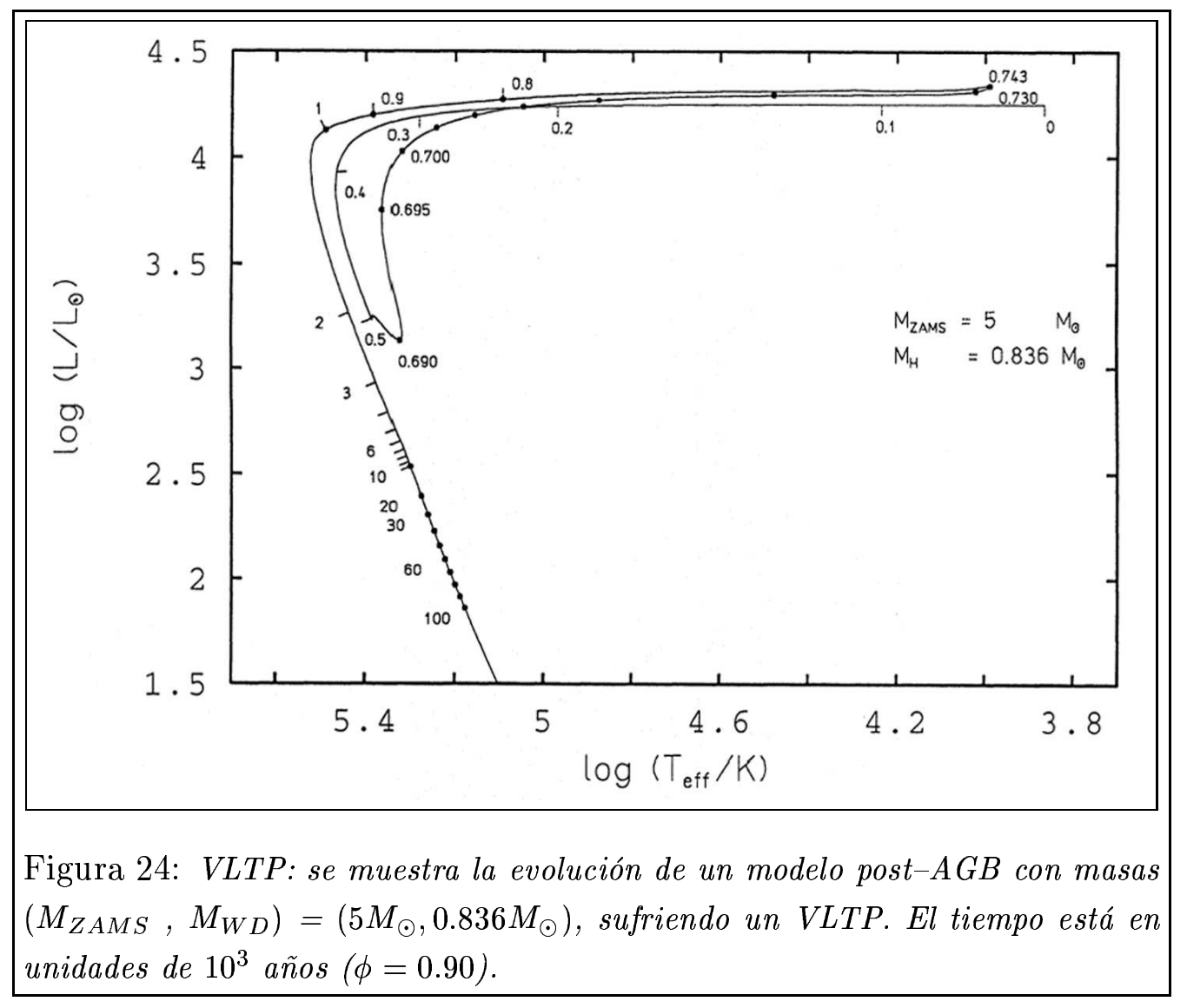

Un cálculo de un VLTP para un modelo de $\left(M_{Z A M S}, M_{W D}\right)=\left(5 M_{\odot}, 0.836 M_{\odot}\right)$ realizado por Blöcker podemos verlo en la fig. (24) (Blöcker, 1995a). Nuestros cómputos de un VLTP se muestran en la fig. (25). En ella vemos la evolución de una estrella con una masa $\left(M_{Z A M S}, M_{W D}\right)=\left(2.7 M_{\odot}, 0.5885 M_{\odot}\right)$ desde la secuencia principal hasta el dominio de las estrellas enanas blancas $\mathrm{DQ}$, incluyendo los estados correspondientes a los pulsos térmicos sobre la AGB y el episodio de born again. Las edades están en $10^{3}$ años, en donde el cero corresponde al pico de la quema de helio del último pulso térmico. 


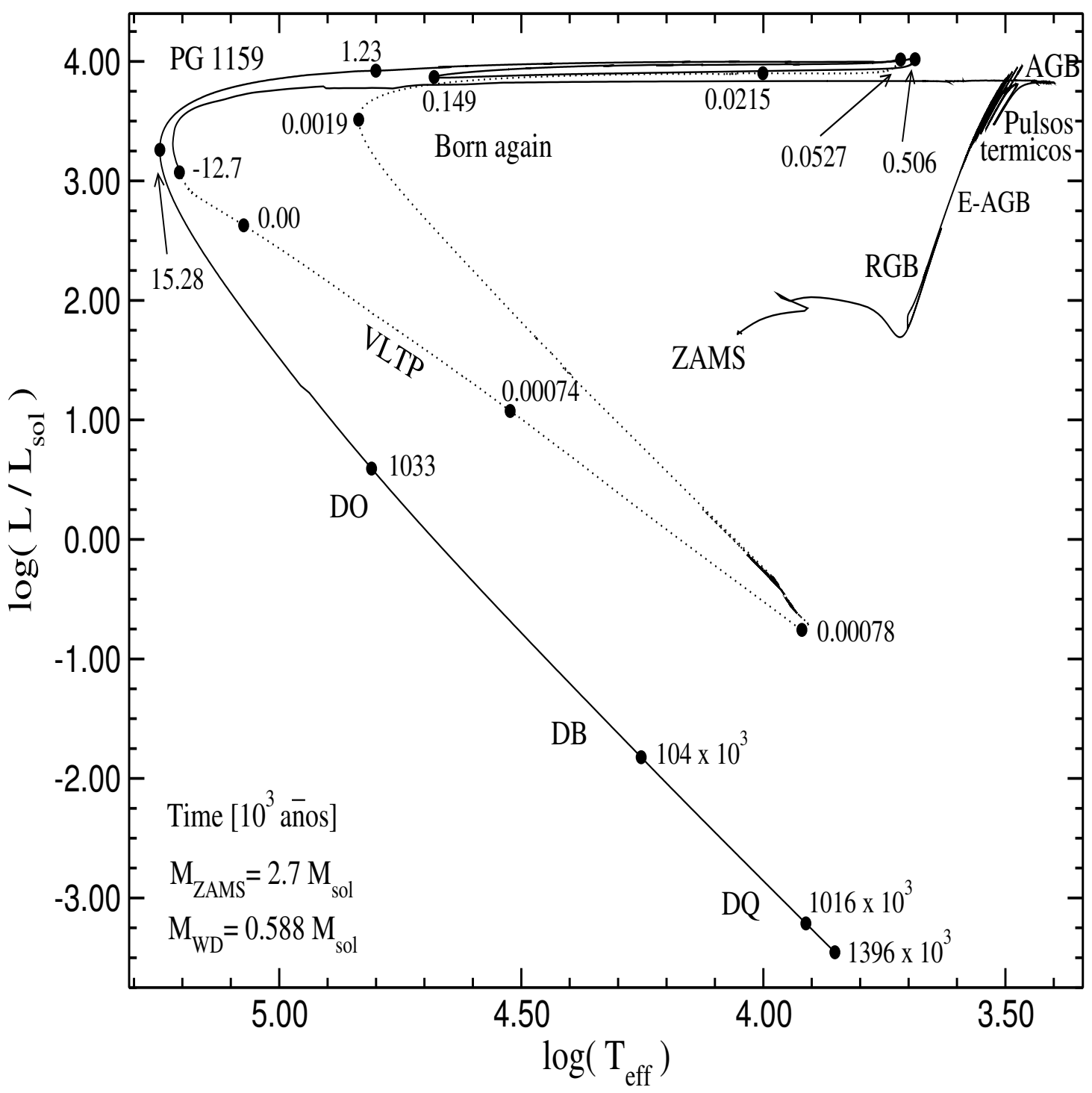

Figura 25: Trayectoria evolutiva sobre el diagrama $H-R$ de una estrella con masa $\left(M_{Z A M S}\right.$, $\left.M_{W D}\right)=\left(2.7 M_{\odot}, 0.5885 M_{\odot}\right)$ que experimenta un VLTP en su fase temprana de enfriamiento después de que la quema de hidrógeno ha cesado. Se muestra la evolución desde la ZAMS, pasando por la fase de los pulsos térmicos, etapa de nebulosa planetaria, fase de 'born again', hasta la rama de enfriamiento de las enanas blancas. Los números sobre la trayectoria indican edades en miles de años donde se ha tomado el cero en el máximo de $L_{H e}$ del último pulso. Se indica el dominio de las estrellas PG1159, DO, DB y DQ, como así también el estado de pulsos térmicos, VLTP y born again. Después del episodio de born again el remanente post-AGB deficiente en hidrógeno experimenta una segunda incursión a la región de temperaturas bajas alcanzando posteriormente la rama de enfriamiento de las enanas blancas. Computada con nuestro código. 
En la fig. (26) se muestran los primeros diez pulsos térmicos que se suceden en la AGB y el pulso térmico final que ocurre fuera de la AGB, para una estrella con masa $\left(M_{Z A M S}, M_{W D}\right)=$ $\left(2.7 M_{\odot}, 0.5885 M_{\odot}\right)$. Se grafican las luminosidades superficiales $L_{\text {sup }}$, la debida a la quema de hidrógeno por el ciclo CNO $L_{C N O}$ y la debida a helio $L_{H e}$, la escala temporal está en millones de años desde la secuencia principal. Se han computado 10 pulsos con un período de interpulso de unos $1.2 \times 10^{5}$ años calculado antes de que el remanente abandone la AGB como resultado de la pérdida de masa. En forma ampliada se grafica el pulso que ocurre fuera de la AGB (el VLTP). Se indica también la fase con la cual la estrella abandonó la AGB $\phi \approx 0.89$. Notar que los picos de los pulsos debido a helio son escalonados y ascendentes. El crecimiento de la luminosidad de helio se debe a que algo de helio de la intercapa es llevado hacia las capas más profundas y más calientes donde es quemado a temperaturas altas, esto es ayudado por el OV de la base de la capa convectiva del flash de helio -ver fig. (19.c).

En la fig. (27) se muestra parte del núcleo de carbono-oxígeno hasta el borde del progenitor como función de la coordenada masa. En el gráfico superior se muestra el perfil químico antes del $1^{e r}$ pulso térmico, esto es al final de la E-AGB; en el inferior se grafica al comienzo del born again en el punto $t=-12.7$, marcado con un círculo negro en la fig. (25).

Vale la pena mencionar que la distribución de las abundancias de carbono y oxígeno dentro del núcleo como la que se muestra en la fig. (27), es típica de situaciones para las cuales se permiten episodios de mezcla adicionales más allá del borde del núcleo convectivo durante la quema central de helio. Tales episodios de mezcla extra, particularmente el overshoot y/o semiconvección nuclear, tienen una gran influencia sobre la distribución de carbono y oxígeno en el núcleo de las enanas blancas. En nuestro modelo cuando el núcleo central de helio cae a cero, el oxígeno central tiene un valor de 0.724 por masa. La importancia del OV durante la quema de helio central, ha sido señalada por Althaus y colaboradores (Althaus et al., 2003) y Córsico y colaboradores (Córsico et al., 2004). Ellos encontraron que, la existencia de un overshooting nuclear durante la etapa de quema central de helio en un progenitor de una enana blanca masiva, muestra características notorias en el espectro teórico pulsacional de las estrellas ZZ Ceti masivas. La masa del núcleo de carbono/oxígeno aumenta considerablemente durante la fase térmicamente pulsante, debido a que la capa que quema helio se mueve hacia afuera. La región del OV se ensancha debajo de la zona convectiva del flash de helio -ver figs. (19) y (20)- hacia el momento del pico del pulso. Entonces el reservorio de helio de la región de la intercapa es llevado hacia adentro de la estrella enriqueciendo con carbono y oxígeno la base de la zona convectiva. Posterior al pulso el OV de la envoltura draga el carbono hacia la superficie. Esto hace que la relación carbono/oxígeno crezca de $\approx 0.25$ (antes de los pulsos) hasta $\approx 0.34$ (después de la fase térmicamente pulsante). Otro resultado de nuestros cálculos es la formación de ${ }^{13} \mathrm{C}$ y ${ }^{14} \mathrm{~N}$ en la base del reservorio de helio, después del final de la fase de dredge up. En este sentido, durante el OV difusivo del 


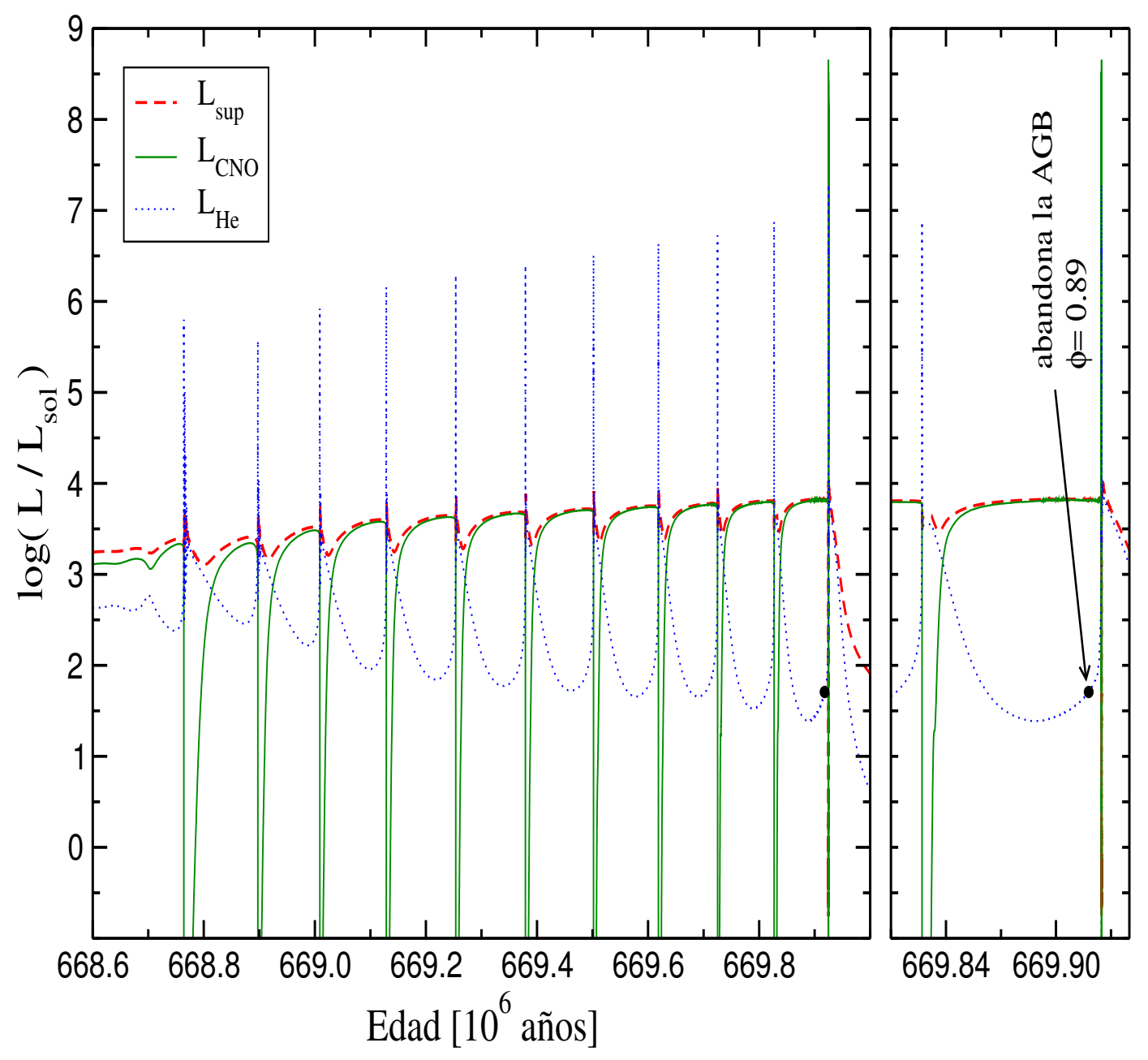

Figura 26: Pulsos térmicos para una estrella de $M_{Z A M S}=2.7 M_{\odot}$. Mostramos, a partir de nuestros resultados, la dependencia temporal de la luminosidad superficial $\left(L_{\text {sup }}\right)$, la luminosidad debida a la quema de hidrógeno por ciclo CNO ( $\left.L_{C N O}\right)$ y la debida a la quema de helio $\left(L_{H e}\right)$ en unidades solares. Después de experimentar 10 pulsos térmicos, el progenitor abandona la AGB, con una fase $\phi=0.89$, indicada en el gráfico con un punto negro. El gráfico de la derecha muestra el último pulso térmico que ocurre en la $A G B$ y el responsable del born again que se sucede en la fase post-AGB (pulso 11) cuando el remanente está en la rama de enfriamiento, ahí la luminosidad $L_{C N O}$ excede los $10^{8} L_{\odot}$. 


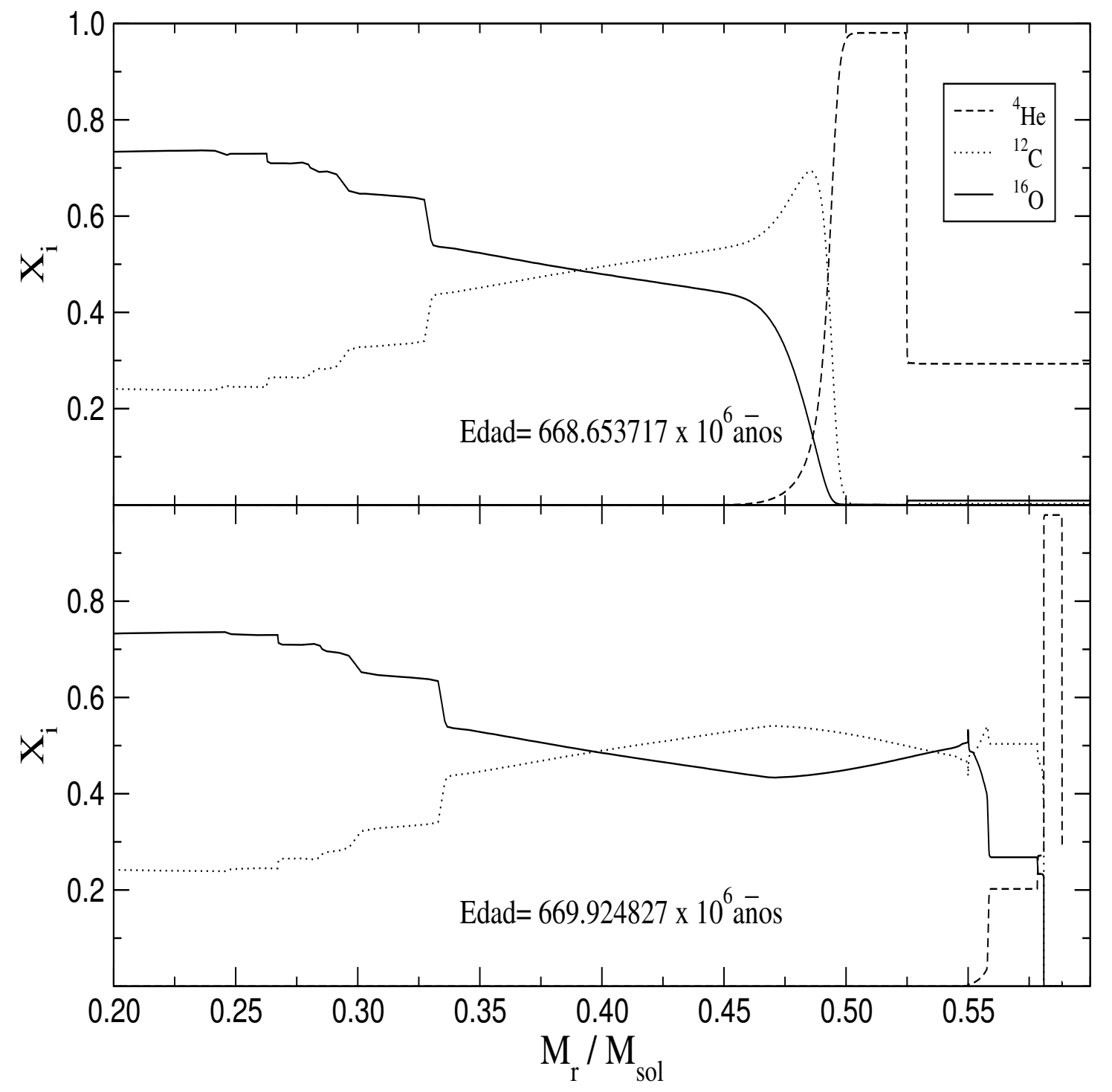

Figura 27: Para un modelo $\left(M_{Z A M S}, M_{W D}\right)=\left(2.7 M_{\odot}, 0.5885 M_{\odot}\right)$, se representa en el gráfico superior, la abundancia de ${ }^{4} \mathrm{He},{ }^{12} \mathrm{C} y{ }^{16} \mathrm{O}$ antes del primer pulso térmico. Ídem en el inferior, para el instante que realiza el primer codo en el azul en el diagrama $H-R$ (es el punto que en la fig. (25) está rotulado con $t=-12.7$ ). 
tercer dredge up se ha formado una región pequeña en la cual el hidrógeno de la envoltura coexiste con el carbono y el nitrógeno, en cantidades apreciables. Cuando esta región se calienta produce la formación de un reservorio de ${ }^{14} \mathrm{~N}$ con una abundancia por masa de 0.45 y un reservorio de ${ }^{13} \mathrm{C}$ con una abundancias de 0.06. Durante el período del interpulso, el reservorio de ${ }^{13} \mathrm{C}$ es quemado radiativamente a temperaturas relativamente bajas (alrededor de $\left.80 \times 10^{6} \mathrm{~K}\right)$ antes de que se queme en el próximo pulso mediante la reacción ${ }^{13} \mathrm{C}(\alpha, n){ }^{16} \mathrm{O}$, la principal fuente de neutrones en las estrellas AGB. Mientras que el ${ }^{14} \mathrm{~N}$ es tragado por la zona convectiva del flash de helio en el próximo pulso térmico y quemado mediante la cadena ${ }^{14} \mathrm{~N}(\alpha, \gamma){ }^{18} \mathrm{~F}\left(\beta^{+}, \nu\right){ }^{18} \mathrm{O}(\alpha, \gamma){ }^{22} \mathrm{Ne}$ que convierte en ${ }^{22} \mathrm{Ne}$ todo el ${ }^{14} \mathrm{~N}$ presente. En acuerdo con Herwig (Herwig, 2000) nuestros cálculos muestran que el OV difusivo de la base de la envoltura convectiva es un proceso que conduce a la formación de un reservorio de ${ }^{13} \mathrm{C}$ $\mathrm{y}{ }^{14} \mathrm{~N}$ que lleva a la formación de elementos pesados a través de procesos de captura lenta de neutrones. 


\section{$7 \quad$ La baja abundancia de hidrógeno}

Como hemos visto en el escenario desarrollado, el VLTP, algunas estrellas centrales de las nebulosas planetarias experimentan un pulso térmico final, después de haber logrado la configuración de una enana blanca y comenzado su descenso a lo largo de la secuencia de enfriamiento de las enanas blancas. Los cálculos teóricos demuestran que durante tal pulso, la mayoría del hidrógeno remanente en la estrella, hacia el encendido del pulso es incorporado dentro de la capa convectiva que quema helio y es quemado por completo. Entonces, luego del pulso, la estrella se expande brevemente hacia las dimensiones de gigante roja. La masa de hidrógeno remanente antes del último pulso térmico es del orden de $7 \times 10^{-5} M_{\odot}$. Vale la pena mencionar que de esta complicada etapa, el born again, existen en la literatura pocas simulaciones numéricas detalladas.

El diagrama $\mathrm{H}-\mathrm{R}$ del último pulso térmico de helio y la siguiente fase de born again se grafica en la fig. (28). Las letras sobre la trayectoria indican ciertos instantes evolutivos. El gráfico incorporado muestra la dependencia temporal de la luminosidad debida a la quema de hidrógeno por el CNO y la quema de helio. Notar que en escalas muy cortas de tiempo la estrella sufre cambios importantes en su estructura. Por ejemplo en 0.4 años la estrella desarrolla luminosidades por la quema de hidrógeno tan elevadas como de $10^{8} L_{\odot}$ (punto C), luego de que los protones fueran tragados por la zona convectiva del flash de helio (punto B). La mayor parte de la quema de hidrógeno de la envoltura ocurre entre los puntos $\mathrm{C}$ y E, en alrededor de un mes. En el punto E después de 0.9 años del pico de helio (punto A) la masa de la envoltura residual de hidrógeno ha sido reducida a $10^{-7} M_{\odot}$. Entre los puntos E y F la quema de hidrógeno se extingue gradualmente. En este momento el hidrógeno remanente en la estrella es de $1.3 \times 10^{-8} M_{\odot}$. Después de 0.6 años el remanente alcanza el punto de máxima temperatura efectiva por primera vez después del flash de helio para una temperatura de $\log T_{\text {eff }}=4.83$. Luego de esto el remanente evoluciona hacia el dominio de las gigantes en un tiempo algo más lento. En efecto la temperatura cae a una temperatura de $10000 \mathrm{~K}$ en un intervalo de tiempo de 20 años, y cae a $5200 \mathrm{~K}$ en alrededor de 50 años (el radio estelar ha aumentado 30.2 y $125 R_{\odot}$, respectivamente).

La fig. (29) representa la trayectoria completa en el diagrama $\mathrm{H}-\mathrm{R}$, de una estrella con una masa $\left(M_{Z A M S}, M_{W D}\right)=\left(2.7 M_{\odot}, 0.5885 M_{\odot}\right)$ que experimenta un VLTP, pasando por todas las etapas evolutivas: Secuencia Principal, la RGB, la E-AGB, la AGB, los pulsos térmicos, el inicio de la fase de nebulosa planetaria, la fase born again, el estado de preenana blanca como estrella PG1159 y finalmente la rama de enfriamiento de las enanas blancas. Los números sobre la trayectoria que acompañan a los círculos negros están en años. El interior estelar de los modelos marcados con círculos negros se grafican en las figs. (31) a (39). 


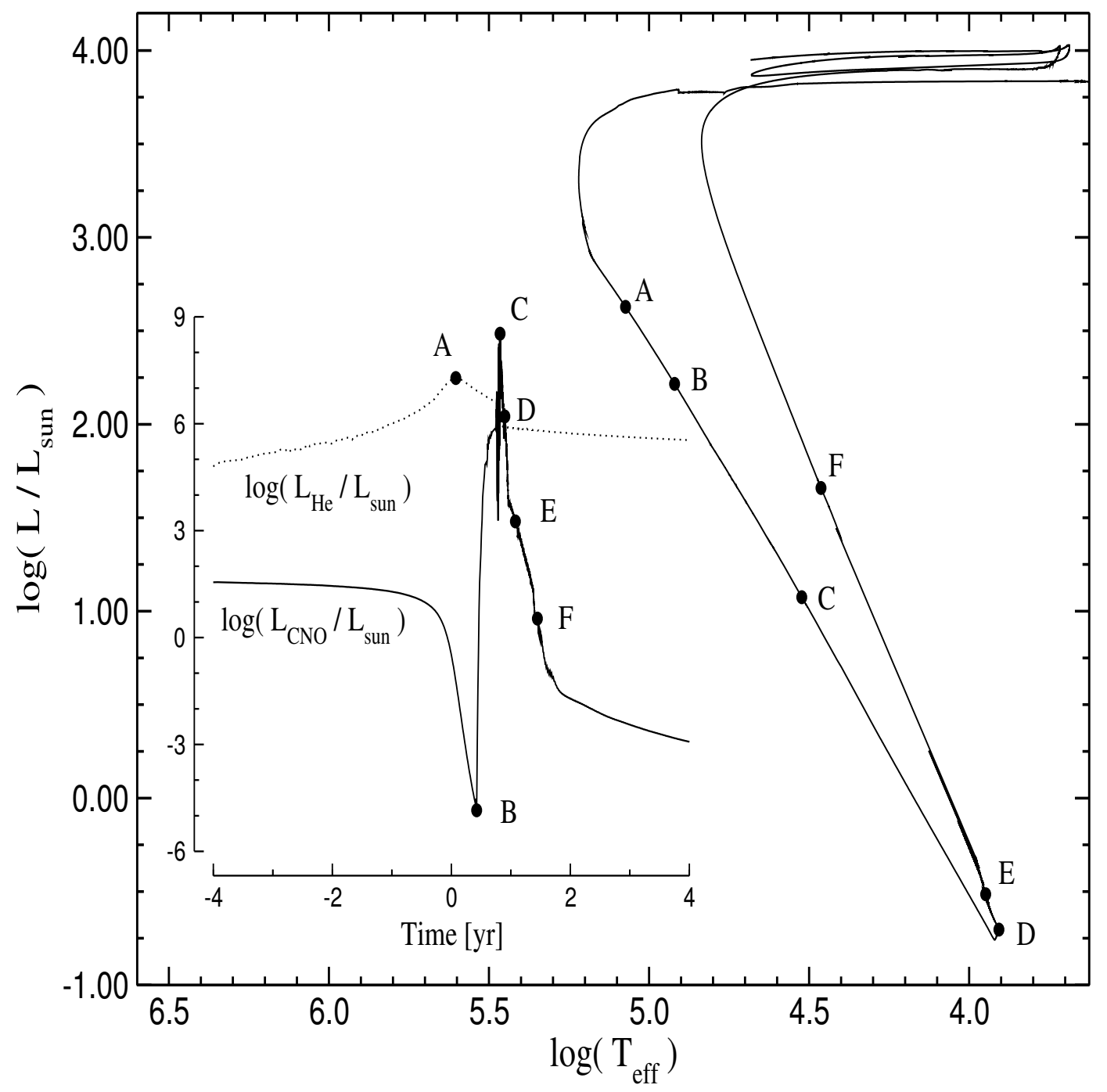

Figura 28: Diagrama $H-R$ de los estados evolutivos de un pulso térmico muy tardío para una estrella con masa $\left(M_{Z A M S}, M_{W D}\right)=\left(2.7 M_{\odot}, 0.5885 M_{\odot}\right)$. El último pulso térmico es el responsable del episodio born again. Notar que el remanente experimenta una segunda incursión (doble loop) hacia la región de las gigantes rojas antes de evolucionar hacia el estado de enana blanca. El gráfico que se incluye muestra la dependencia temporal de la quema de hidrógeno por el ciclo CNO y por el helio (línea llena y punteada, respectivamente). Las letras seleccionadas indican: $A$ y $C$ corresponden al pico de la quema de helio $\left(L_{H e}\right)$ y al pico de la quema de hidrógeno por el ciclo CNO, respectivamente. En el punto B los protones comienzan a ser tragados y quemados por la zona convectiva del flash de helio. Entre los puntos $E$ y $F$, la quema de hidrógeno se vuelve casi extinta, y el hidrógeno remanente alcanza el valor $1.3 \times 10^{-8} M_{\odot}$. 


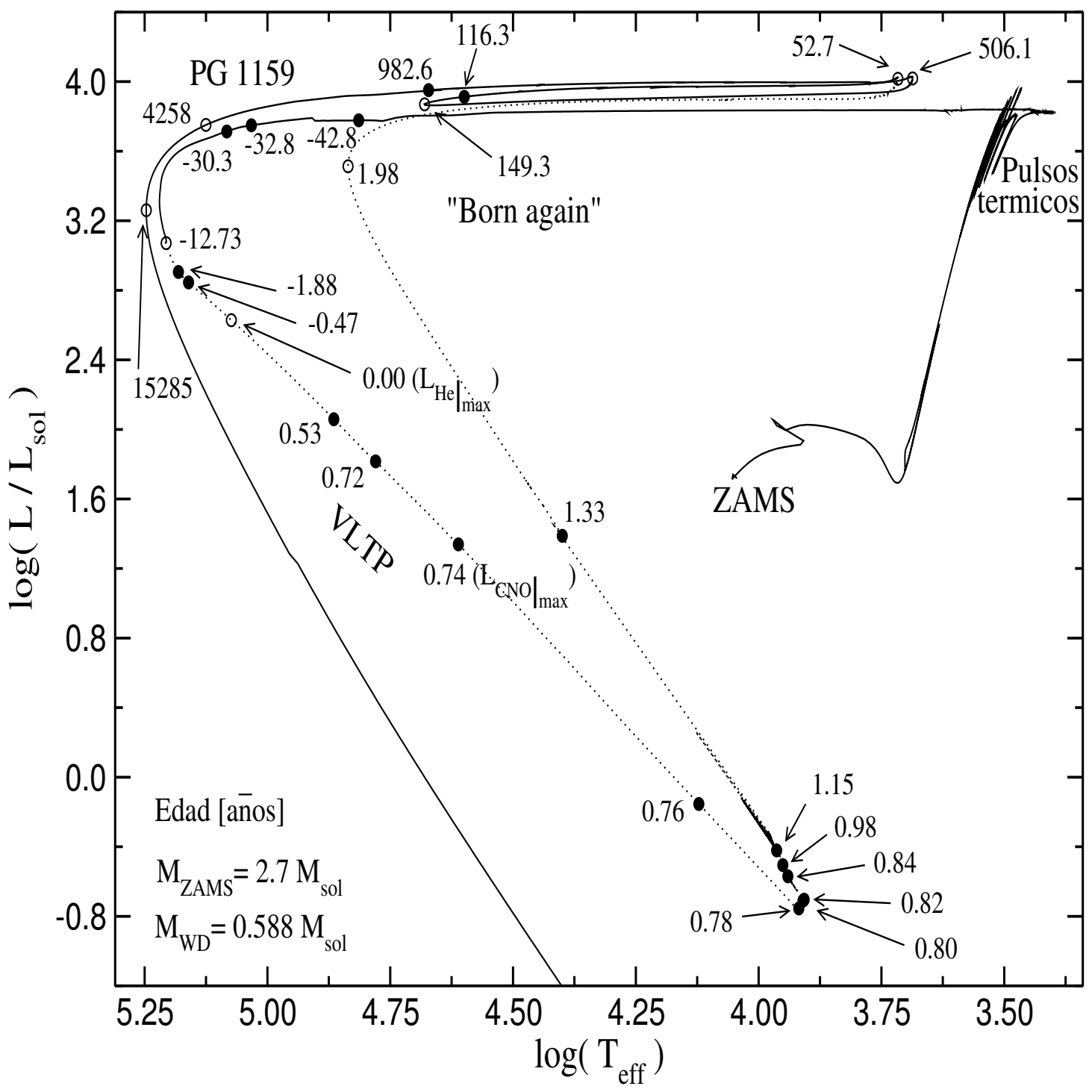

Figura 29: Trayectoria completa de una estrella con $\left(M_{Z A M S}, M_{W D}\right)=\left(2.7 M_{\odot}, 0.5885 M_{\odot}\right)$. Se indican con círculos negros los distintos tiempos los cuales se corresponden a las figs. (31) a (39). Los círculos blancos representan instantes en determinados puntos característicos de la evolución. El cero se ha tomado en el máximo de la $L_{H e}$ (en el mismo lugar que en la fig. (25)). Obtenida de nuestros resultados. 
El círculo blanco con $t=0.00$ es precisamente en el momento en que la luminosidad de helio $\left(L_{H e}\right)$ es máxima. El doble loop en el diagrama $\mathrm{H}-\mathrm{R}$ es un resultado de la penetración de la zona convectiva conductora del flash de helio dentro de las capas ricas en hidrógeno. Cuando los protones son llevados por convección a regiones lo suficientemente calientes son capturados por los núcleos de ${ }^{12} \mathrm{C}$. El consumo de protones por la zona convectiva continúa hasta que la tasa por generación de energía por captura de protones se vuelve comparable a aquella de la quema de helio. La entropía producida por la captura de protones fuerza a la separación de la zona convectiva en dos zonas convectivas distintas: una mantenida por la quema de hidrógeno y la otra por la quema de helio. La expansión inicial es producida por la capa que quema hidrógeno. Cuando esta energía se agota, la envoltura se contrae. La segunda expansión de la envoltura es producida por la capa de helio contigua. Cuando esta fuente de energía se agota, la envoltura se contrae nuevamente y la estrella retorna al dominio de las enanas blancas.

En la fig. (30) se muestran las luminosidades integradas $L_{H e}$ y $L_{C N O}$ para el último pulso. Los tiempos sobre los círculos grises son algunos de los mismos indicados en el diagrama $\mathrm{H}-\mathrm{R}$ de la fig. (29) y corresponden a los interiores estelares de las figs. (31) a (38). Desde el punto en el diagrama $\mathrm{H}-\mathrm{R}$ rotulado con $t=0.00$ hasta que se convierte nuevamente en gigante roja, nuestro modelo demora $\approx 52.7$ años. Luego la estrella procede a quemar el helio durante una escala de tiempo larga, desandando en el diagrama $\mathrm{H}-\mathrm{R}$ aproximadamente, el mismo camino que el seguido mientras quemó el hidrógeno durante la excitación inicial de la nebulosa, la cual se ha expandido considerablemente.

En las figs. (31) a (39) se grafican varios instantes evolutivos en donde los tiempos dados corresponden a los círculos negros de la fig. (29). En las mismas se muestra las variaciones de las abundancias químicas.

Específicamente se observa como cambian las abundancias por masa de ${ }^{1} \mathrm{H},{ }^{4} \mathrm{He},{ }^{12} \mathrm{C}$, ${ }^{13} \mathrm{C},{ }^{14} \mathrm{~N}$ y ${ }^{16} \mathrm{O}$ en función de la fracción de masa $q$. Las regiones grises y sombreadas representan los dominios de la convección y el OV, respectivamente. En la fig. (33) superior $(t=-0.47)$, muestra la estratificación química al comienzo del pulso térmico de helio. En las capas más externas, la composición química corresponde a aquella dada por los episodios de dredge up durante la fase AGB. En la capa de puro helio, la abundancia relativamente grande de ${ }^{14} \mathrm{~N}$ refleja la eficiencia de la quema de hidrógeno en las fases evolutivas previas en el procesamiento de ${ }^{12} \mathrm{C}$ y ${ }^{16} \mathrm{O}$ en ${ }^{14} \mathrm{~N}$. Gracias a la gran cantidad de energía resultante de la quema de helio, se va desarrollando una zona convectiva del flash de helio que crece hacia afuera. En aproximadamente 1 año el borde exterior de esta zona convectiva alcanza la base de la envoltura rica en hidrógeno -fig. (33) inferior $(t=0.53)$. Como resultado los protones comienzan a ser transportados hacia adentro, hacia capas más calientes ricas en carbono, donde son capturados mediante la reacción ${ }^{12} \mathrm{C}(p, \gamma){ }^{13} \mathrm{~N}$. Como resultado de una 


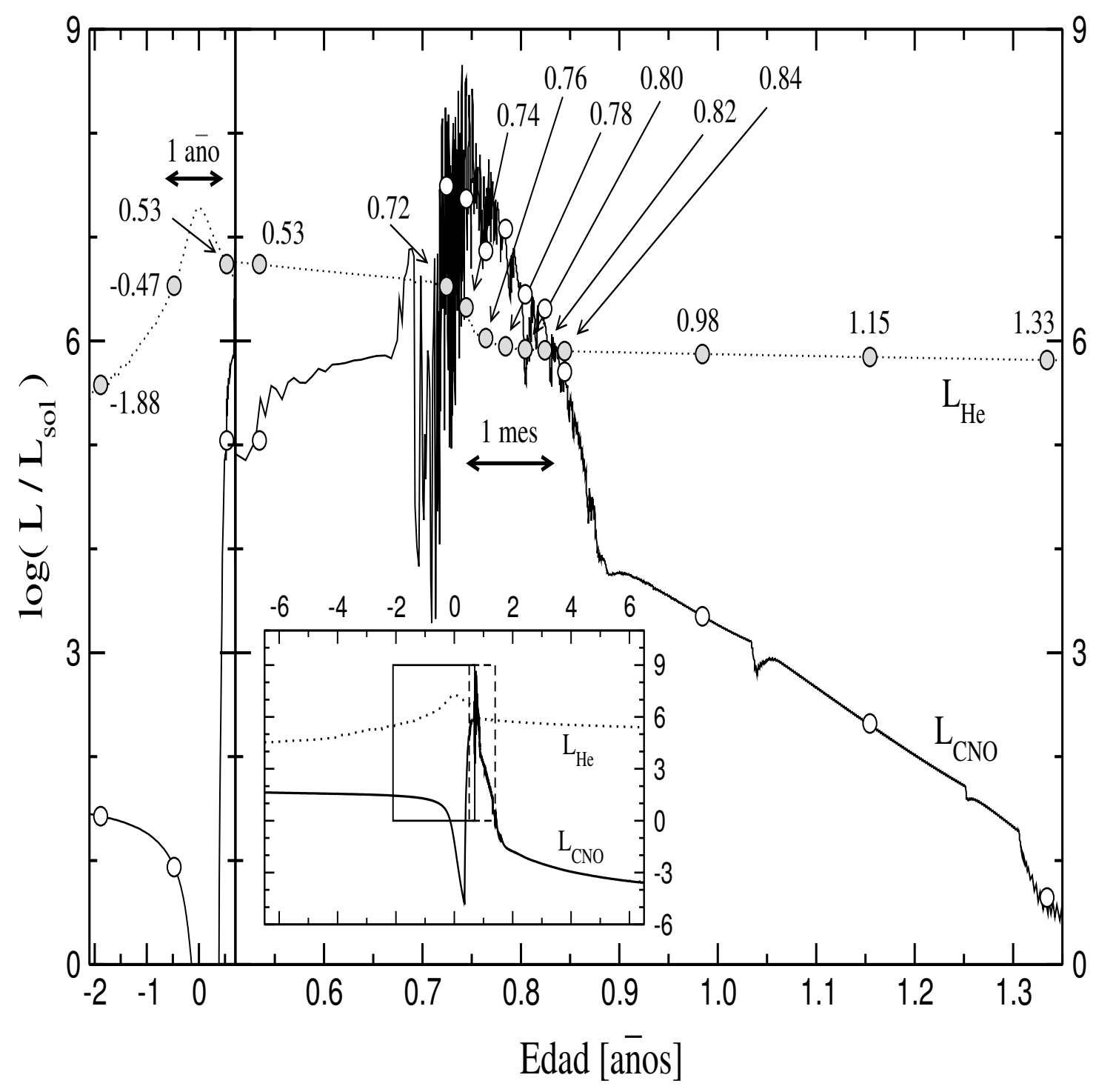

Figura 30: Para una estrella con masa $\left(M_{Z A M S}, M_{W D}\right)=\left(2.7 M_{\odot}, 0.5885 M_{\odot}\right)$ y para el último pulso encargado de generar el "born again", se grafican las luminosidades integradas $L_{H e}$ y $L_{C N O}$. Los círculos grises y blancos representan los tiempos dados en la fig. (29). Los tiempos que se indican sobre los círculos grises son los mismos que para los círculos blancos con igual abscisa. En el gráfico del recuadro inferior se indica que parte del pulso se muestra en forma ampliada. 

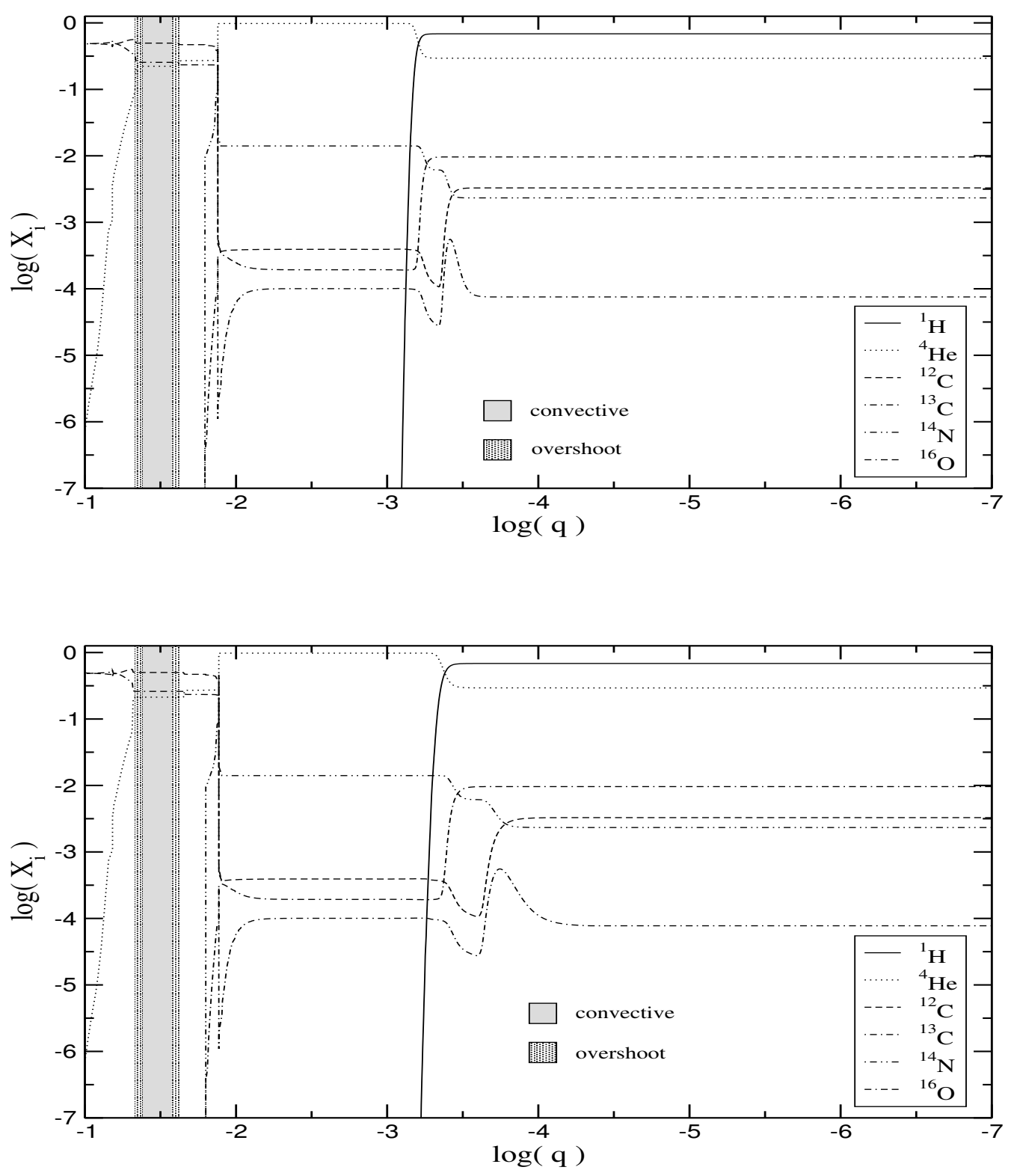

Figura 31: Interiores de los modelos estelares donde se indican las abundancias de ${ }^{1} H$, ${ }^{4} \mathrm{He},{ }^{12} \mathrm{C},{ }^{13} \mathrm{C},{ }^{14} \mathrm{~N}$ y ${ }^{16} \mathrm{O}$ como así también las regiones convectivas y el overshooting. Se grafica $\log q\left(\equiv \log \left(1-\frac{m_{r}}{M_{*}}\right)\right)$ vs. $\log X_{i}$, con $X_{i}$ : abundancia fraccional. El modelo superior corresponde $a t \simeq-42.8$ años y el inferior $a t \simeq-32.8$ años. 

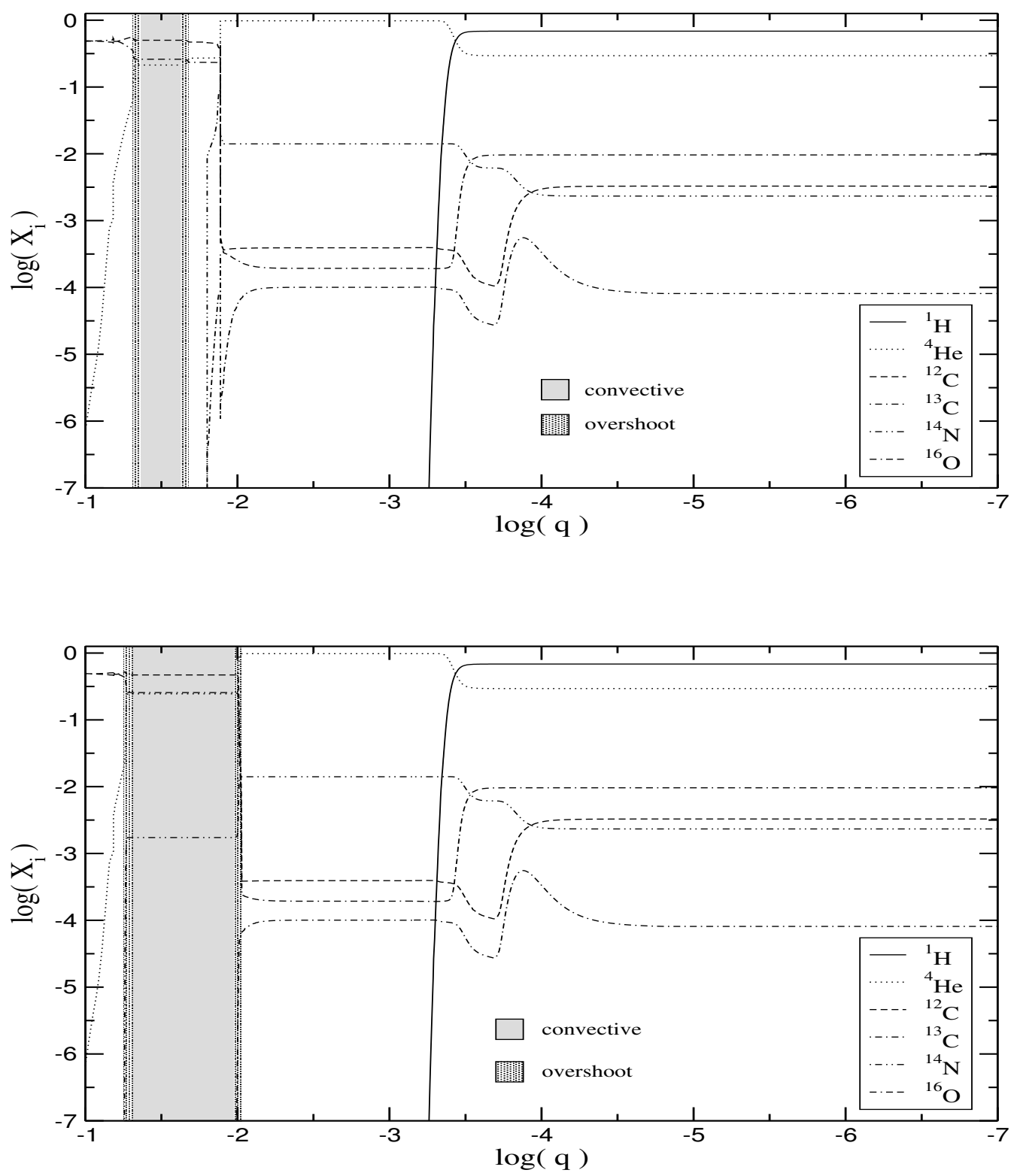

Figura 32: Idem fig. (31). El gráfico superior e inferior corresponde para $t \simeq-30.3$ y -1.88 años respectivamente. Observar el avance de la región convectiva. 

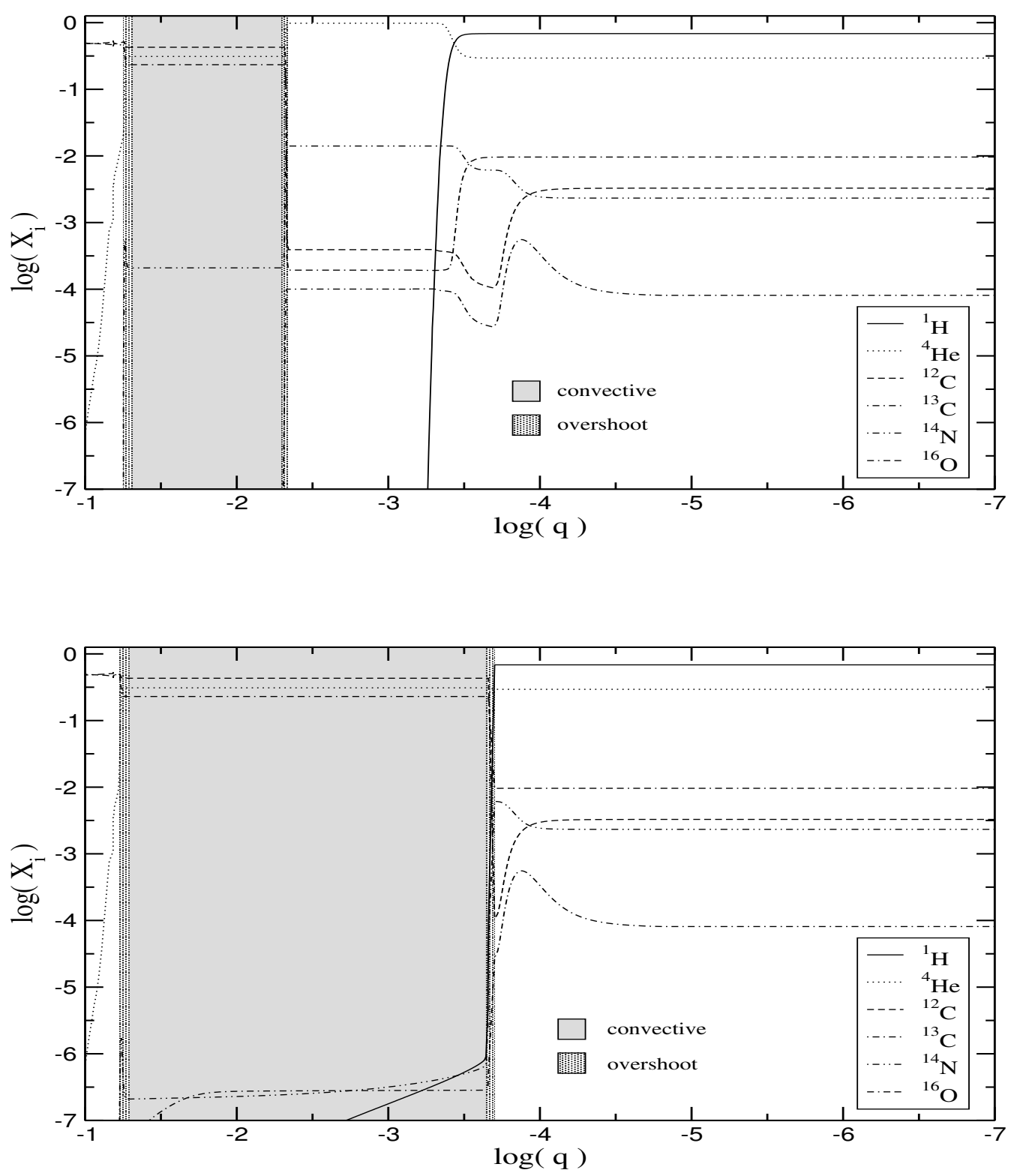

Figura 33: Idem fig. (31). El gráfico superior e inferior corresponde $a t \simeq-0.47$ y 0.53 años respectivamente. La región convectiva avanza hacia afuera transformando los perfiles internos de abundancias. 

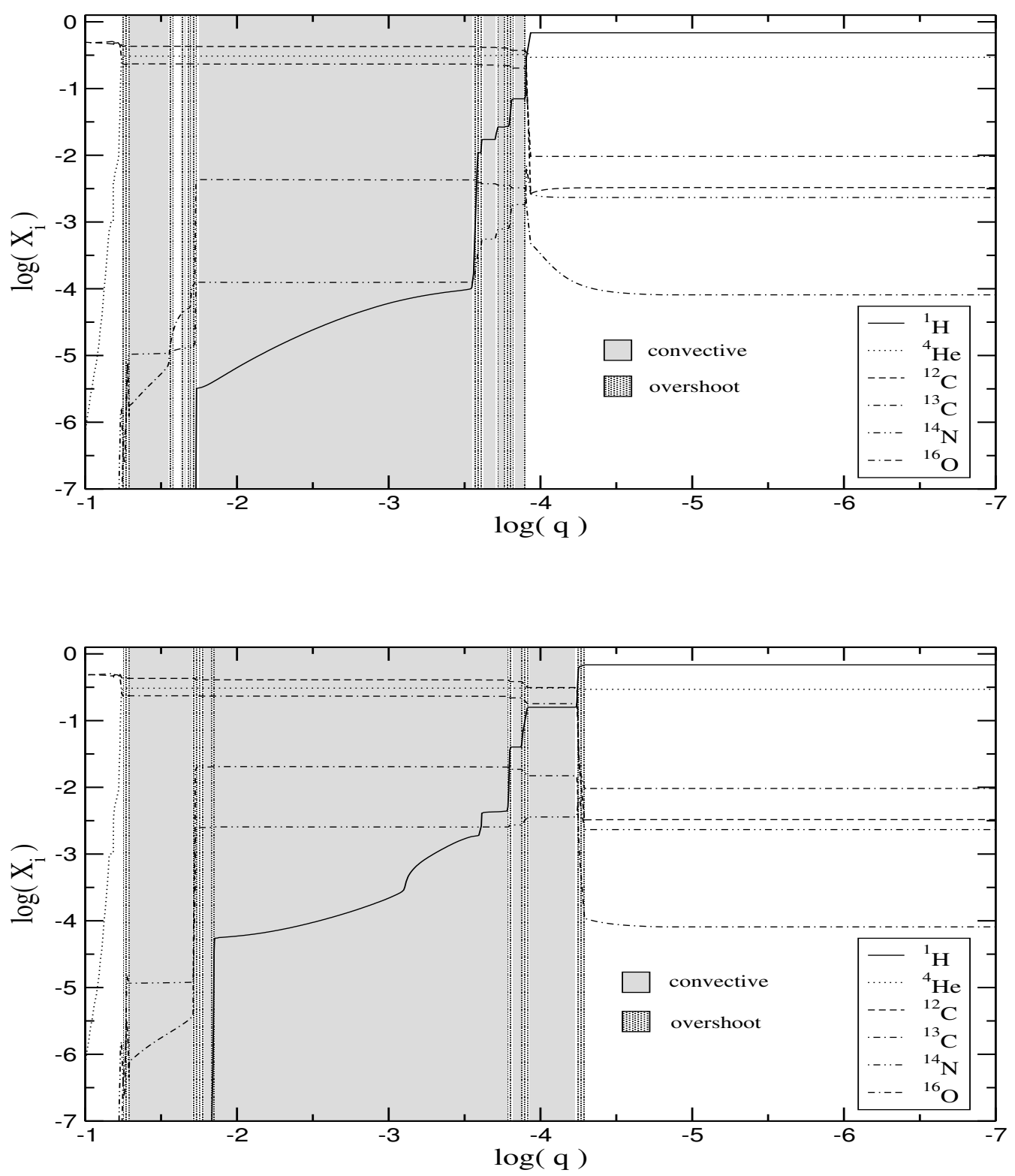

Figura 34: Idem fig. (31). El gráfico superior e inferior corresponde para $t \simeq 0.72$ y 0.74 años respectivamente ( $\Delta t \simeq 7.3$ días). El tiempo 0.74 es el del máximo de la luminosidad $L_{C N O}$. 

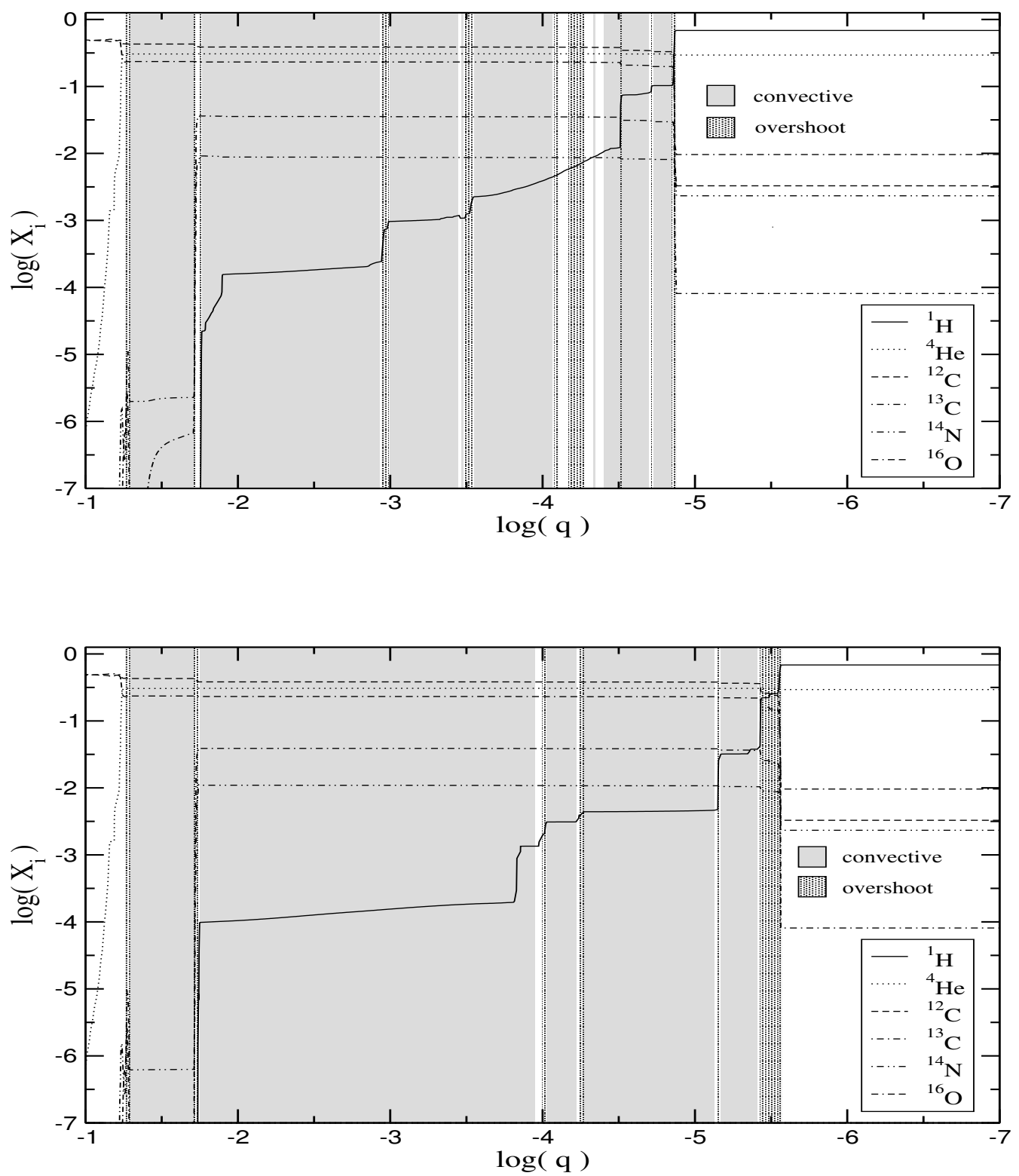

Figura 35: Idem fig. (31). El gráfico superior e inferior corresponde para $t \simeq 0.76$ y 0.78 años respectivamente $(\Delta t \simeq 7.3$ días $)$. 

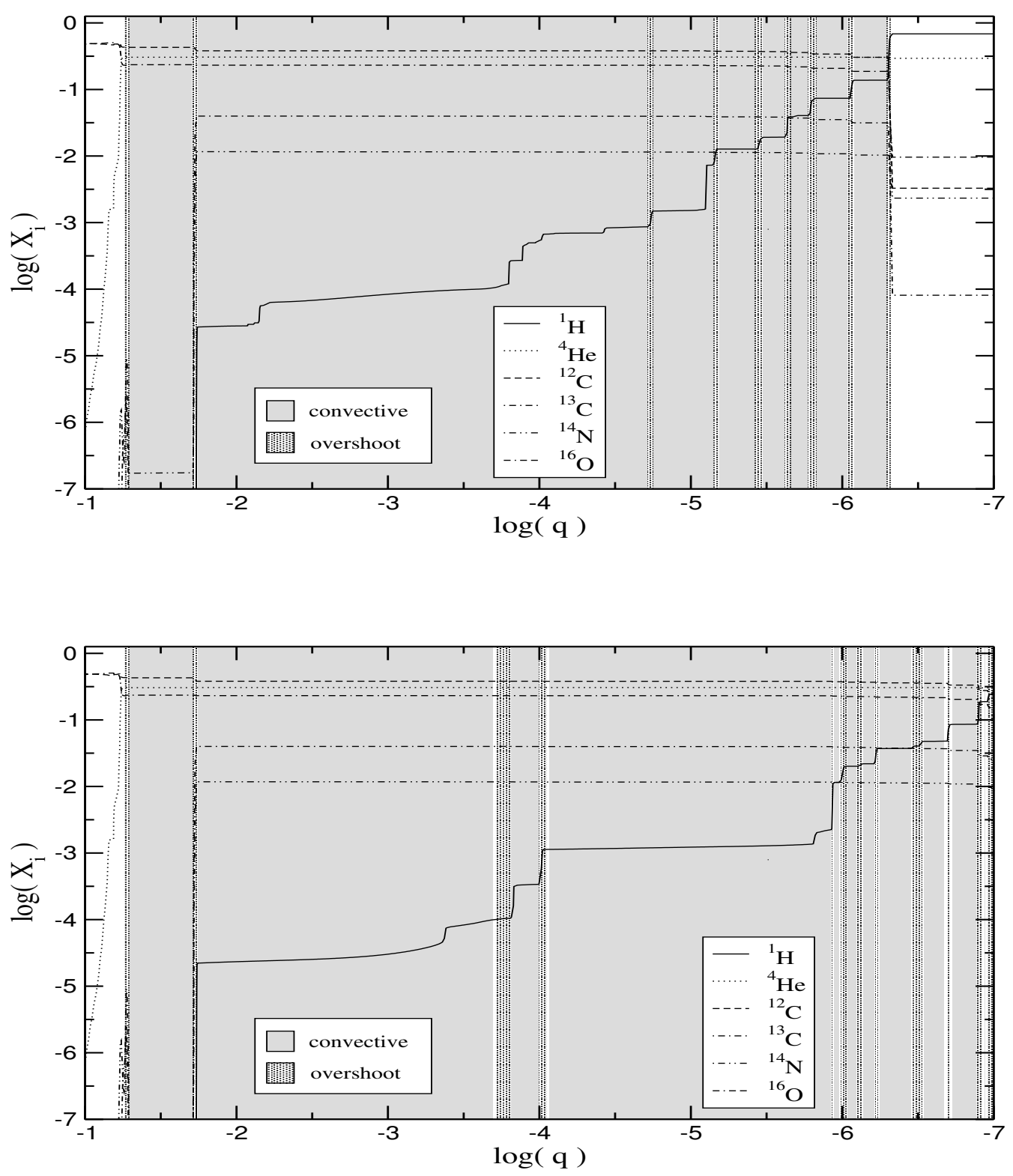

Figura 36: Idem fig. (31). El gráfico superior e inferior corresponde para $t \simeq 0.80$ y 0.82 años respectivamente ( $\Delta t \simeq 7.3$ días). La región convectiva alcanzó la superficie, la estrella está en el vértice inferior y avanzando hacia el born again. El hidrógeno superficial comienza a disminuir para el modelo del gráfico inferior. 

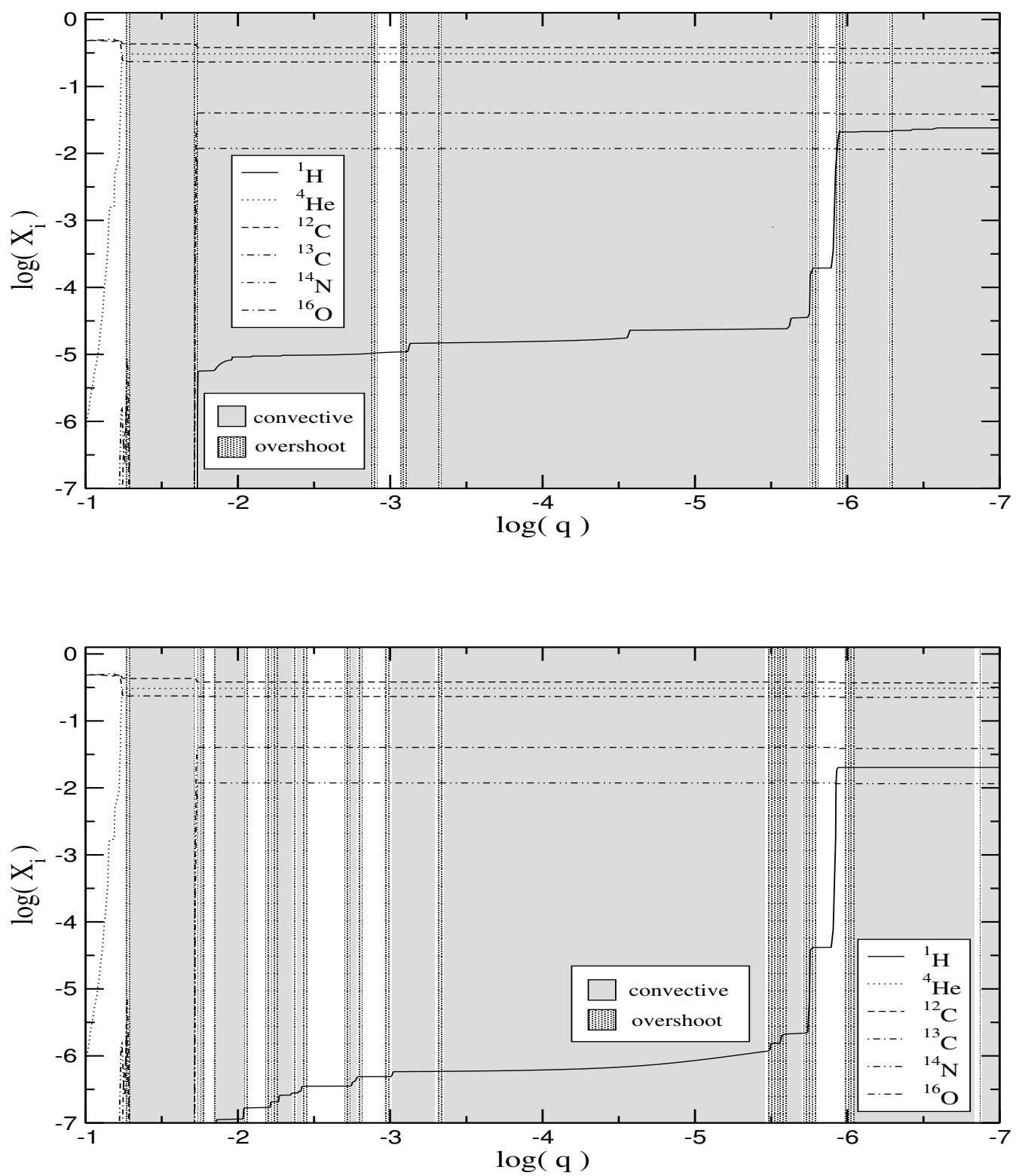

Figura 37: Idem fig. (31). El gráfico superior e inferior corresponde para $t \simeq 0.84$ y 0.98 años respectivamente $(\Delta t \simeq 51.1$ días $)$. El hidrógeno superficial es $X_{H} \simeq 0.018$. 

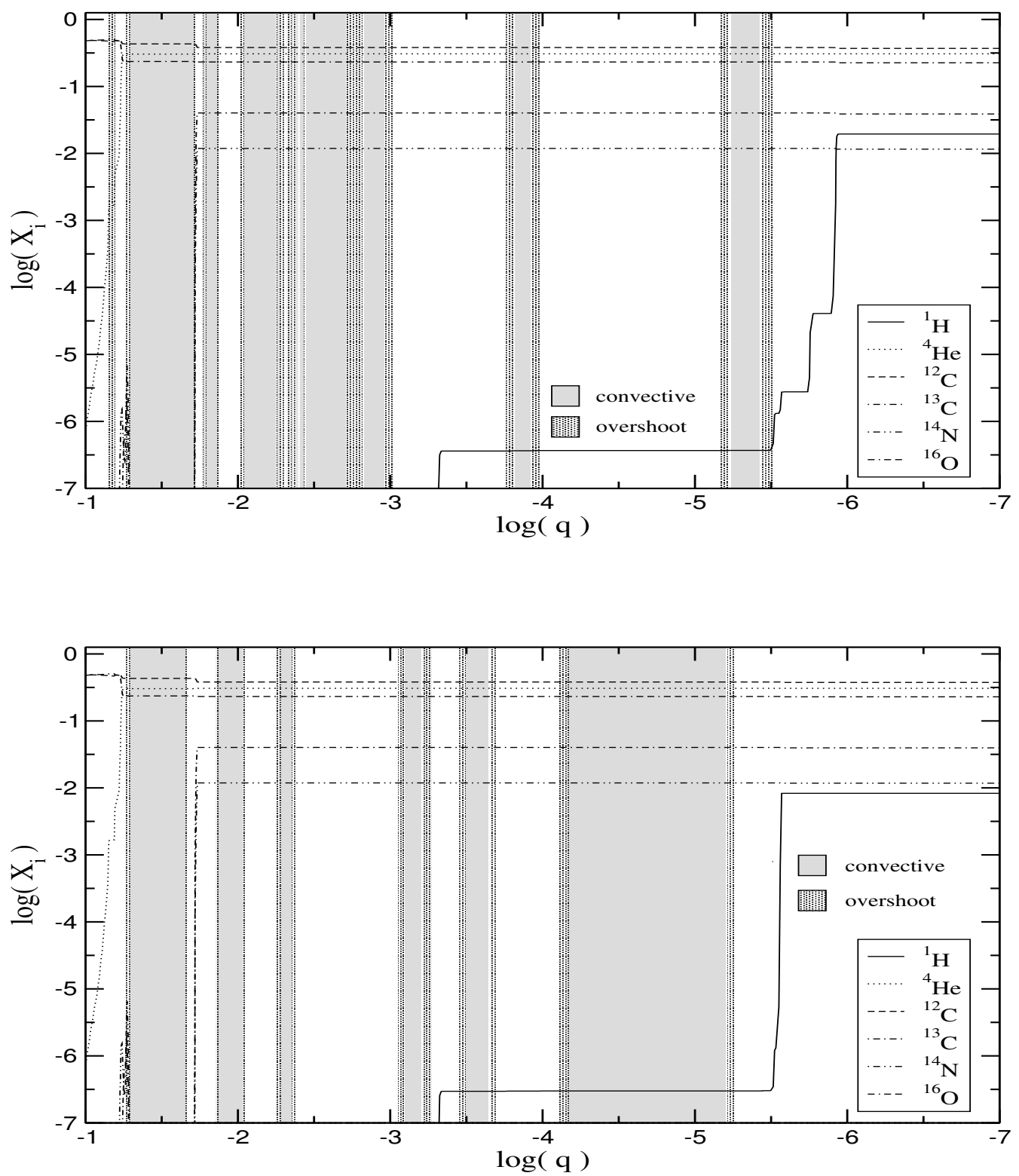

Figura 38: Idem fig. (31). El gráfico superior e inferior corresponde para $t \simeq 1.15$ y 1.33 años respectivamente $(\Delta t \simeq 65.7$ días $)$. El hidrógeno superficial a $t \simeq 14.07$ es $X_{H} \simeq 0.008$. 

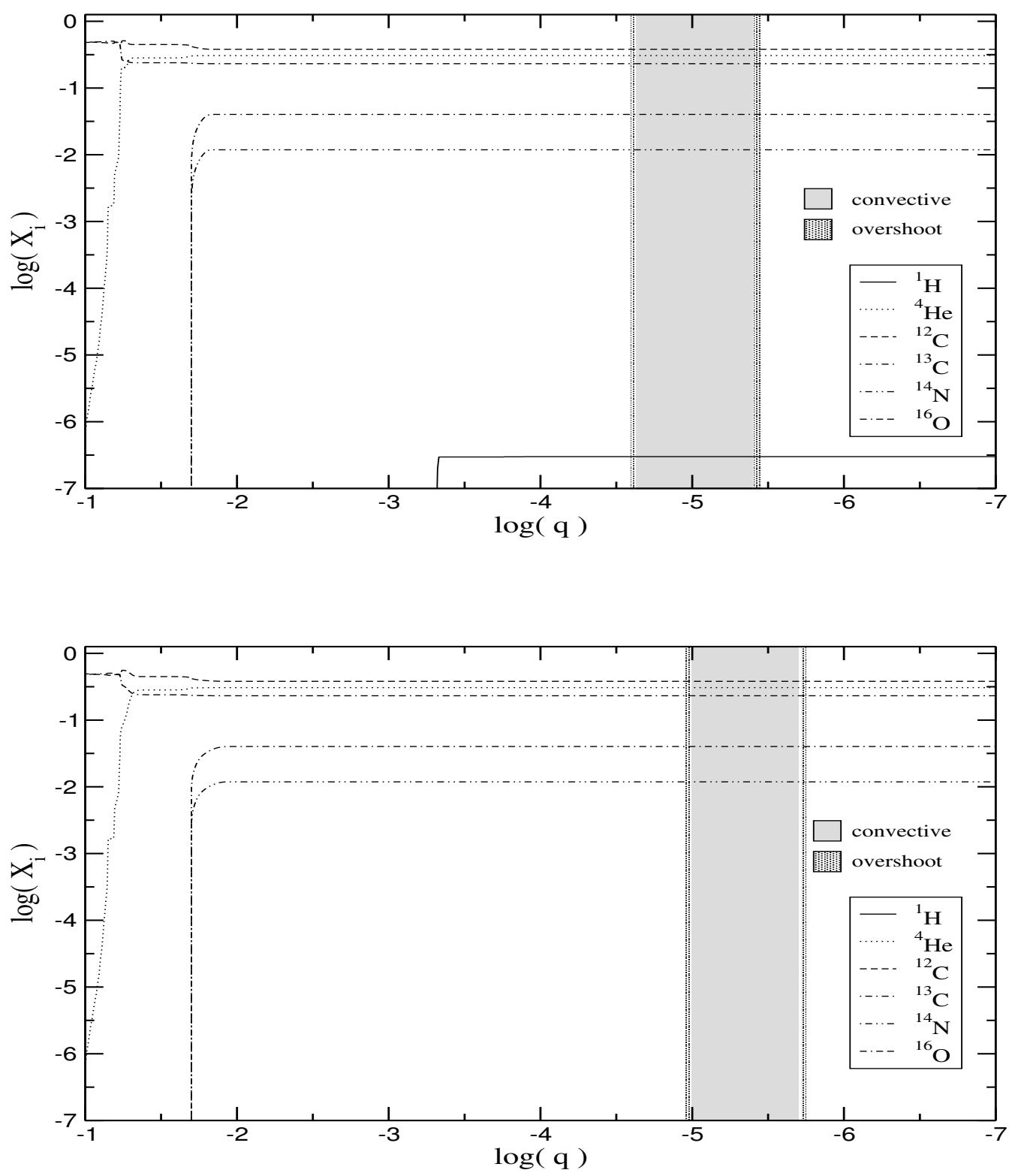

Figura 39: Idem fig. (31). El gráfico superior e inferior corresponde para $t \simeq 116.3$ y 982.6 años respectivamente. En el grafico superior es $X_{H} \simeq 3 \times 10^{-7}$ y en el inferior el hidrógeno superficial ha sido agotado casi por completo. 
fuerte quema de hidrógeno una zona radiativa y de salt finger pequeña se establece hacia $\log q \approx 1.6$, la cual separa la zona convectiva original en dos zonas convectivas distintas. Esta situación está ilustrada en la fig. (34) superior $(t=0.72)$. La quema de hidrógeno y helio toma lugar en la base de tales zonas convectivas. Notar la gran cantidad de ${ }^{13} \mathrm{C}$ en la zona convectiva mantenida por la quema de hidrógeno (zona convectiva superior). En la zona caliente convectiva del flash de helio el ${ }^{13} \mathrm{C}$ (y el ${ }^{14} \mathrm{~N}$ ) es destruido por la captura $\alpha$ mientras son liberados neutrones. Notar que en estas etapas es de importancia un tratamiento simultáneo de mezcla y quema como se puede apreciar en el perfil de hidrógeno. En la fig. (35) superior $(t=0.76)$, se observa que el borde exterior de la zona convectiva superior se propaga hacia afuera en masa respecto a lo ocurrido para $t=0.72$. Este desplazamiento hacia afuera, mezcla protones de la envoltura original sin procesar. Notar que se mantiene la región salt finger que separa las dos zonas convectivas y que ocurre un aumento de ${ }^{13} \mathrm{C}$ y ${ }^{14} \mathrm{~N}$ en la zona convectiva superior, debido a la ingesta de protones. Para $t=0.84-$ fig. (37) superior- la convección llega hasta la superficie, produciendo una zona inestable convectiva extendida desde la zona de quema de protones hasta la superficie estelar. Posteriormente la quema de hidrógeno se extingue -fig. (38) inferior $(t=1.33)$ - y el hidrógeno remanente en este momento es de $\approx 1.310^{-8} M_{\odot}$.

\subsection{Abell 30 y Abell 78}

Dos ejemplos de estrellas que se hallan en la etapa del post-pulso, son las estrellas centrales de las nebulosas planetarias Abell 30 y Abell 78. Ambas se encuentran en la fase de quema quieta de helio y muestran evidencia de eyección de materia rica en helio, desde las superficies de sus estrellas centrales. Su ubicación en el diagrama H-R está acotada por ( $R_{n e b}$ es el radio de la nebulosa circundante):

- Abell 30: $4.85 \lesssim \log T_{\text {eff }} \lesssim 5.0, \quad 2.7 \lesssim \log L / L_{\odot} \lesssim 3.1, \quad R_{\text {neb }} \approx 5300 \mathrm{pc}$

- Abell 78: $4.83 \lesssim \log T_{\text {eff }} \lesssim 4.9, \quad 3.1 \lesssim \log L / L_{\odot} \lesssim 3.3, \quad R_{n e b} \approx 4500 \mathrm{pc}$

Se han estimado las pérdidas de masa, para las estrellas Abell 30 y Abell 78, mostrando altas abundancias de helio y carbono características del material de la intercapa, el cual aparece en las superficies de las estrellas centrales cuando ellas están en la fase extendida de quema de helio. Para Abell 30 (Hazard et al., 1980) y Abell 78 (Ford and Jacoby, 1983) se encuentran abundancias con una relación $\mathrm{He} / \mathrm{H}$ de 20. La historia previa para estas estrellas es la siguiente: durante el pulso térmico final, la mayor parte de hidrógeno es absorbida por la capa convectiva que quema helio, entonces hay suficiente energía nuclear que es convertida en energía térmica durante el pulso. El exceso de energía térmica es 
transformado en energía potencial gravitacional, lo que hace que la estrella se expanda hacia dimensiones de gigante. El modelo se lanza sobre una fase de quema quieta de helio, durante la cual la estrella vuelve casi sobre la trayectoria que había atravesado durante la fase final de quema de hidrógeno. Ya previamente el remanente había eyectado material desde su superficie, que luego de lograr una temperatura lo suficientemente alta, lo enciende como nebulosa planetaria. Las estrellas de Abell 30 y Abell 78 tienen un espectro de WR y despliegan fuertes perfiles P Cygni en el ultravioleta, ambas características dan la certeza de pérdida de masa y elevadas luminosidades estelares. La ausencia de hidrógeno en sus superficies puede deberse a tres posibles escenarios (Iben et al., 1983), descriptos en las secciones (7.1.1), (7.1.2) y (7.1.3).

\subsubsection{Dilución y quema de hidrógeno}

El hidrógeno, es literalmente "tragado" por la capa convectiva que quema helio durante el pico del último pulso térmico. Éste es llevado hacia adentro de la estrella por convección (y por lo tanto es diluido "en abundancia") hacia regiones de temperaturas y densidades lo suficientemente altas. Comenzará entonces una quema mediante la reacción ${ }^{12} \mathrm{C}(p, \gamma){ }^{13} \mathrm{~N}$. La energía liberada por ésta y las reacciones resultantes puede conducir al desdoblamiento de la zona convectiva original en dos zonas convectivas separadas por una delgada zona radiativa, con quema de helio en la base de la capa convectiva interior y quema de hidrógeno en la base de la capa convectiva exterior. En la base de la capa convectiva, la conversión del ${ }^{12} \mathrm{C}$ en ${ }^{13} \mathrm{~N}$ depende tanto de la relación de protones y ${ }^{12} \mathrm{C}$ hacia el comienzo de la fase de quema en la capa, como de la temperatura (Iben et al., 1983):

i) Si la concentración de protones es inicialmente menor que la del ${ }^{12} \mathrm{C}$ la quema culminará con la formación de ${ }^{13} \mathrm{C}$; pero no se formará ${ }^{14} \mathrm{~N}$ nuevo, aunque el ${ }^{14} \mathrm{~N}$ preexistente en la capa convectiva externa sobrevivirá.

ii) Si la abundancia de protones excede por lejos a la del ${ }^{12} \mathrm{C}$, las reacciones:

$$
{ }^{13} \mathrm{C}(p, \gamma){ }^{14} \mathrm{~N}(p, \gamma){ }^{15} \mathrm{O}\left(e^{+}, \nu\right){ }^{15} \mathrm{~N}(p, \alpha){ }^{12} \mathrm{C}
$$

formarán ${ }^{14} \mathrm{~N}$ y ${ }^{4} \mathrm{He}$ frescos.

iii) En cualquier otro caso, hacia el final de la fase de quema de hidrógeno la estrella quedará con una capa superficial agotada en hidrógeno, consistiendo entre otros de ${ }^{4} \mathrm{He},{ }^{12} \mathrm{C},{ }^{13} \mathrm{C}$ y ${ }^{14} \mathrm{~N}$. 
Si el número de protones introducidos en la capa excede al número de núcleos de ${ }^{12} \mathrm{C}$, el resultado neto será una relación de abundancias de N/C $>1$.

El hidrógeno podría quemarse hasta agotarse en una escala de tiempo corta comparada a la fase del pico de quema de helio, la capa convectiva que quema helio puede extenderse nuevamente hacia afuera dentro de la región que está experimentando quema de hidrógeno. En consecuencia el ${ }^{13} \mathrm{C}$ puede ser barrido dentro de la capa convectiva y desaparecerá mediante la reacción ${ }^{13} \mathrm{C}(\alpha, n){ }^{16} \mathrm{O}$, y los neutrones liberados producirán isótopos ricos en neutrones, los que serán distribuidos en toda la capa convectiva. La característica de este proceso de adición de neutrones será una función sensible de ambos de la relación ${ }^{14} \mathrm{~N} /{ }^{13} \mathrm{C}$ en la capa y de la temperatura efectiva para la cual se quema el ${ }^{13} \mathrm{C}$. Cuando el ${ }^{14} \mathrm{~N} /{ }^{13} \mathrm{C} \gtrsim 1$, la reacción ${ }^{14} \mathrm{~N}(n, p){ }^{14} \mathrm{C}$ impondrá una restricción sobre el número de neutrones posibles para ser capturados por núcleos pesados. La escala de tiempo de liberación de neutrones es esencialmente el tiempo de vida del ${ }^{13} \mathrm{C}$ con respecto a la reacción ${ }^{13} \mathrm{C}(\alpha, n){ }^{16} \mathrm{O}$ (Iben et al., 1983).

\subsubsection{Dilución convectiva superficial}

Se debe a un avance de la zona convectiva superficial, hacia adentro de la estrella, la cual aparece y crece cuando la estrella se expande hacia dimensiones de gigante posterior al pico del pulso. Por ejemplo para un modelo estelar de $0.6 M_{\odot}$, la base de la zona convectiva superficial comienza a extenderse hacia adentro a través de la capa rica en hidrógeno, pero en este caso logra entrar sólo hasta un máximo en masa de $10^{-5} M_{\odot}$ (Iben et al., 1983), por lo tanto fallaría en alcanzar la base de la envoltura rica en hidrógeno. Sin embargo, el proceso considerado en la sección (7.1.1) puede reducir la capa rica en hidrógeno, la cual sobrevive al "tragado" convectivo durante el pico del pulso hasta alcanzar una masa menor que $10^{-5} M_{\odot}$, en tal caso la convección superficial durante la fase gigante será efectiva en diluir esta capa con un procesamiento total del material en su interior.

\subsubsection{Eyección debida al viento}

Este mecanismo se debe a un viento que opera, durante la alta luminosidad de la fase de quema quasiestática de helio. Gracias a la presencia de perfiles P Cygni en los espectros de las estrellas centrales de las nebulosas planetarias, puede inducirse la pérdida de masa occurrida por conducción del viento. Las tasas de pérdidas de masa son del orden de $\sim 10^{-10} M_{\odot} / a \tilde{n} o$ hasta $\sim 10^{-6} M_{\odot} / a \tilde{n} o$. La pérdida de masa durante la fase AGB puede influir en la escala de tiempo evolutiva de las estrellas centrales de nebulosas planetarias. Esta pérdida podría afectar levemente la masa de la envoltura residual rica en hidrógeno en el encendido del flash final. La pérdida de masa puede remover los últimos vestigios del hidrógeno de la superficie, después del pulso térmico cuando la estrella vuelve sobre sus pasos o cercano a ellos en el 
diagrama H-R. Si no se tienen en cuenta los procesos dados en las secciones (7.1.1) y (7.1.2), para obtener una superficie carente de hidrógeno requiere que $\sim 5 \times 10^{-5} M_{\odot}$ del material sea removida en una escala de tiempo de $\sim 10^{4}$ años, dando una tasa de pérdida de masa de solamente $\gtrsim 5 \times 10^{-9} M_{\odot} /$ años. Esto está de acuerdo con las estimaciones existentes de tasas de pérdidas de masa.

\subsubsection{Mecanismos posibles para explicar el nitrógeno superficial}

Los tres mecanismos descriptos en las secciones (7.1.1), (7.1.2) y (7.1.3) tienen en común que predicen como resultado una abundancia superficial enriquecida en helio y en carbono. Pero difieren en la abundancia de nitrógeno. Los dos mecanismos descriptos primero, la conversión termonuclear de hidrógeno superficial en helio durante el pico del pulso y el proceso de dilución convectiva durante la fase gigante siguiendo al pico del pulso, son compatibles con la presencia de una abundancia de nitrógeno observable. En cambio, la pérdida de masa occurrida por conducción del viento durante la fase de quema quieta de helio dejará al descubierto la materia de la intercapa la cual no contiene nitrógeno (durante el pulso térmico, el nitrógeno es totalmente destruido en la intercapa).

La mayoría de los núcleos de nebulosas planetarias del tipo WR pertenecen a la subclase WC, con ninguna línea de nitrógeno. Sin embargo en Abell 30 y Abell 78 sus marcados perfiles P Cygni muestran que el nitrógeno está presente en la atmósfera de sus estrellas centrales (clasificadas como estrellas WCN), esto sería evidencia para: dilución y procesamiento termonuclear de hidrógeno durante el pico del pulso y/o dilución por la convección superficial durante la fase gigante siguiente al pico del pulso. 


\section{Escenarios born again}

En esta sección describiremos las características de otros escenarios born again, como el de los objetos Sakurai y las implicaciones del esquema del transporte convectivo en los tiempos evolutivos.

\subsection{Objetos Sakurai: otra evidencia observacional de born again}

Los objetos Sakurai, como V4334 Sagittarii, son estrellas que sufren un born again AGB posterior a un pulso térmico muy tardío (VLTP). Son objetos que pasan desde un estado de pre-enana blanca al de una gigante en unos pocos años. Una masa estelar alta no puede ser la causa de su rápida evolución. En cambio, la escala de tiempo evolutiva es reproducida en modelos estelares mediante la aceptación de que la eficiencia para la mezcla de los elementos en la zona convectiva del flash de helio durante un pulso térmico muy tardío, es menor que la predicha por la mixing length theory (MLT). Como resultado de esto, la generación principal de energía a partir de la captura de protones ocurre cerca de la superficie y la expansión al estado de gigante es acelerado en pocos años.

\subsubsection{La historia evolutiva de un objeto Sakurai: V4334 Sgr}

El objeto Sakurai, la variable V4334, ha desarrollado una evolución dramáticamente rápida tanto en sus parámetros estelares como en abundancia química [(Duerbeck et al., 1997); (Duerbeck et al., 2000)]; (Asplund et al., 1999)].

Hacia 1976, posiblemente detectado por el relevamiento ESO/SERC cerca del límite de detección de $m_{j}=21$ (Pollacco, 1999), la cual coincide con los parámetros estelares de una pre-enana blanca en el diagrama de H-R -ver fig. (40) (Herwig, 2001b).

La última vez que no se detectó fue en 1994 para el límite de magnitud $m_{v}=15.5$ y la primer detección posterior fue para $m_{v}=12.4$ por K. Takamizawa en 1995 -ver (Duerbeck et al., 1997)-, esto representa una rigurosa restricción sobre la velocidad evolutiva -fig. (40). Esta evolución ha sido interpretada como el resultado de un flash de helio final que ocurrió en 1994. Hacia comienzos de 1996, la estrella había alcanzado $\log \left(L / L_{\odot}\right) \simeq 3.8$ y se enfrió hasta bien por debajo de $\log \left(T_{e f f}\right) \sim 4$. Desde entonces, la estrella ha continuado enfriándose e iluminándose. De esta manera, de acuerdo a la evidencia observacional, V4334 Sgr debe haber completado la evolución born again desde el estado de pre-enana blanca hasta su apariencia actual como una gigante en alrededor de 2 años. En nuestro modelo de born again, éste posee una masa de $M \simeq 0.5885 M_{\odot}$ y pasó de enana blanca al estado de gigante en $\approx 52$ años -fig. (25).

De acuerdo a las observaciones se ha establecido una escala de tiempo evolutiva muy corta 


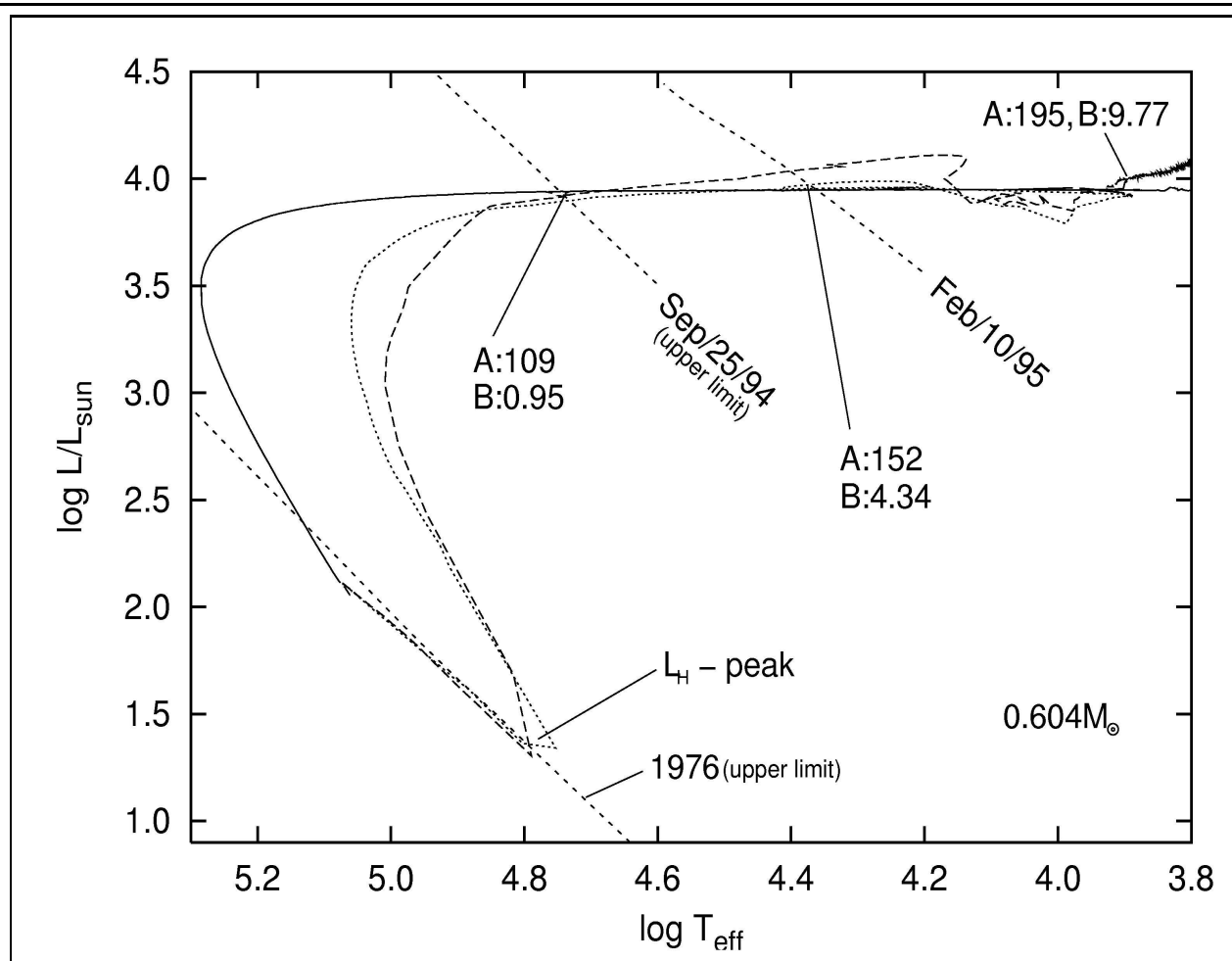

Figura 40: Diagrama $H-R$ de la secuencia post-AGB para una estrella de $0.604 M_{\odot}$ de Herwig et al. (1999a;línea llena) con el VLTP y la subsiguiente evolución born again recalculada con $f_{v}=3$ (línea punteada, rotulada con “A:") y $f_{v}=30$ (línea de segmentos, rotulada como "B:"). El tiempo dado para $\log T_{\text {eff }}=3.9$ corresponde para el primer espectro presentado en Asplund et al. (1999) con fecha del 20 de abril de 1996. Todos los tiempos indicados están en años y dan el tiempo evolutivo desde el momento del pico de la gran luminosidad de la quema de hidrógeno $L_{H}$. Las rectas de trazos cortos representan tres magnitudes diferentes debajo de las cuales ha sido observada V4334 Sgr en diferentes momentos. En esta figura, se ha asumido que $d=4 \mathrm{kpc}$ y la luminosidad considerada es la de la trayectoria evolutiva de la estrella de $0.604 M_{\odot}$. Hay dos rectas segmentadas en donde se indican como límite superior (upper limit) debido a que V4334 Sgr ha sido vista por debajo o cerca del límite de detección. (Herwig, 2001)

con una estrella central de nebulosa planetaria (CSPN) aparentemente normal, lo que hace que falte una explicación teórica que aclare tal fenómeno. Se podrían invocar dos escenarios diferentes posibles de flashes finales: LTP y VLTP. El primero de ellos, el LTP, es aplicable a FG Sagittae. El pulso térmico ocurre mientras la estrella está todavía sobre el tramo horizontal de la trayectoria cuando está cruzando desde la fase AGB a la fase de CSPN a 
luminosidad constante. Durante la primer fase post-AGB, la capa de hidrógeno está aún encendida y esto evita la mezcla del material de la envoltura dentro de la zona convectiva del flash de helio, durante el pulso térmico. La evolución born again es energéticamente conducida por el flash de helio. Para estos modelos LTP, el intervalo de tiempo $\left(\tau_{B A}\right)$ desde que ocurre el LTP/VLTP hasta la vuelta a la AGB es de alrededor de 100-200 años [(Blöcker, 1995a); (Schönberner, 1979)]. Esto está en total desacuerdo con lo observado en V4334 Sgr.

Tanto la detección posible de ESO/SERC en 1976 como los modelos de fotoionización indican que el pulso térmico post-AGB ha ocurrido demasiado tarde, cuando la estrella se había aproximado a la secuencia de enfriamiento de las enanas blancas. En este caso, de un VLTP, la quema de hidrógeno se ha frenado; luego los protones en la envoltura son mezclados hacia abajo y dentro de la zona convectiva del flash de helio donde son quemados en una escala de tiempo convectivo. Puesto que la quema nuclear y la mezcla convectiva ocurren en la misma escala de tiempo y en la misma ubicación debe emplearse un tratamiento especial.

El VLTP desarrollado por Herwig y colaboradores (Herwig et al., 1999a) tiene en cuenta la generación de energía nuclear por captura de protones en la zona convectiva del flash de helio. Ellos encuentran que $\tau_{B A} \sim 350$ años. Este modelo no está de acuerdo con V4334 Sgr. Para que se obtengan tiempos cortos para este tipo de born again debe cumplirse que la eficiencia de mezcla de los elementos en la zona convectiva del flash de helio, es reducida comparada con la velocidad de mezcla predicha por la mixing length theory (MLT).

\subsection{2 ¿Cómo se reproduce un modelo de un objeto Sakurai?}

Si en un modelo VLTP, el pico de energía liberada por la captura de protones está localizado en una zona profunda en donde está la zona convectiva del flash de helio, la entropía aumenta en esas capas por una luminosidad adicional por quema de hidrógeno, que apenas afecta las capas más exteriores. Esto es debido a que en la zona convectiva del flash de helio, la temperatura es muy elevada por el actual flash de helio. Si los protones son capturados a tal profundidad en la región de la intercapa, la energía liberada correspondiente es simplemente una perturbación de la prominente inestabilidad del flash de helio. Entonces, la ingestión de protones no cambia en forma significativa las escalas evolutivas de tiempo del born again. Como resultado la evolución born again siguiente a un VLTP será (similar a la evolución born again después de un LTP) del orden de unos pocos cientos de años. Esto no es compatible con las escalas de tiempo observadas de la evolución del born again de la V4334 Sgr.

La posición del pico de la quema de hidrógeno está determinada por la competencia entre las escalas de tiempo de mezcla $\left(\tau_{m e z c}\right)$ y nuclear $\left(\tau_{n u c}\right)$. El coeficiente de difusión dado para el tratamiento dependiente con el tiempo para un elemento de mezcla convectivo está dado 
por $D_{M L T}=\frac{1}{3} \alpha_{M L T} \cdot H_{p} \cdot v_{M L T}$, donde $v_{M L T}$ es la velocidad de mezcla convectiva de acuerdo a la MLT (Langer et al., 1985) y la cantidad $\ell_{p}=\alpha_{M L T} \cdot H_{p}$ es la longitud de mezcla. La escala de tiempo nuclear $\tau_{n u c}$ decrece con el aumento de la temperatura así como la tasa de reacción de la captura de protones por el ${ }^{12} \mathrm{C}$, aumenta. La principal generación de energía para una quema convectiva rápida de protones, ocurrirá para la posición en que la zona convectiva del flash de helio cumple con $\tau_{n u c} \simeq \tau_{m e z c}$. Esta posición se mueve hacia la parte superior de la zona convectiva del flash de helio, si la eficiencia convectiva de mezcla de los isótopos se reduce. Entonces es de esperarse que la velocidad de la evolución del born again después de un VLTP, dependa de la posición de la liberación de energía dentro de la estrella.

Una manera de reducir la eficiencia de mezcla convectiva podría ser mediante el cambio del parámetro de longitud de mezcla $\alpha_{M L T}$. Sin embargo, esto afecta tanto al transporte de energía convectivo como así también lo que le sucede. Se define entonces un nuevo parámetro $F_{v}=D_{M L T} / D_{C M}$, donde $D_{C M}$ es el coeficiente de difusión para la mezcla de la composición. Los cálculos realizados por Herwig 2001, fueron para secuencias de modelos VLTP y born again con valores de $F_{v}=1,3,30$, y 300 .

Los modelos con velocidad convectiva reducida para la mezcla de la composición, no sólo evolucionan más rápido sino también presentan una evolución modificada para las zonas convectivas. En los cálculos con la eficiencia de mezcla convectiva reducida, la quema de hidrógeno toma lugar en la región superior de la zona convectiva de la intercapa y establece su propia capa convectiva por encima de la actual zona convectiva del flash de helio. En contraste, la quema de hidrógeno, del caso en donde no se altera el coeficiente de difusión, sucede en un lugar más profundo dentro de la región de la intercapa, y la zona convectiva separada de quema de hidrógeno tiene una vida muy corta dentro de la zona convectiva del flash de helio.

\subsubsection{Comparación con las observaciones}

La eficiencia reducida de la mezcla de la composición conduce a una evolución diferente de las zonas convectivas. Esto afecta a los elementos $\mathrm{CNO}$ y sus razones isotópicas. La relación superficial ${ }^{12} \mathrm{C} /{ }^{13} \mathrm{C}$ para el modelo born again con $f_{v}=30$ es de $\sim 5$ y así está de acuerdo con las observaciones de Asplund et al. (1999).

Como se vio la evolución born again posterior al VLTP es sensiblemente dependiente de la mezcla de la composición debida a la convección. Los cómputos realizados por Herwig muestran que la velocidad convectiva en la zona convectiva del flash de helio, decrece con la masa estelar de la estrella post-AGB. Mientras que la estrella con masa $0.604 M_{\odot}$ tiene una velocidad $v_{M L T} \sim 3 \mathrm{~km} \mathrm{~s}^{-1}$, la de $0.535 M_{\odot}$ muestra sólo una $v_{M L T} \sim 0.35 \mathrm{~km} \mathrm{~s}^{-1}$. Esto hace que el modelo de menor masa evolucione más rápido en su vuelta a la AGB. Esta rápida 
evolución debe atribuirse a la baja velocidad convectiva.

El modelo born again para V4334 Sgr que mejor ajusta es el de $F_{v} \sim 100$, es decir la eficiencia del transporte del material por convección es más pequeña que la predicha por la MLT en un factor de $\sim 100$ (Herwig, 2001b). Con ese valor de $F_{v}$ Herwig ha reproducido el tiempo evolutivo observado.

\subsection{Tratamiento de la convección}

Como hemos visto nuestro tiempo de escala de born again $(\sim 52$ años desde el pico de helio hasta el extremo rojo en la primera incursión a la fase gigante) es mayor que el obtenido por Herwig para el objeto Sakurai V4334 Sgr ( 2 años $)$. Otros cálculos evolutivos realizados por Herwig (Herwig, 2001b) están basados en la MLT standard, la cual predice tiempos evolutivos grandes de born again $(\sim 350$ años $)$ para una estrella de $0.604 M_{\odot}$. Como vimos en las secciones precedentes el tiempo de la evolución born again puede ser reducido si se disminuye la eficiencia de mezcla en la zona convectiva del flash de helio en un factor 100. La baja eficiencia de mezcla hace que la mayor captura de protones por el ${ }^{12} \mathrm{C}$ ocurra cerca de la superficie estelar por encima de la zona convectiva de quema de helio, con la consecuencia que la expansión hacia la región de las gigantes se produzca en pocos años. Aunque nuestros tiempos evolutivos son mayores que los observados en los objetos Sakurai, son menores en un factor $\sim 7$ a los calculados por Herwig (Herwig, 2001b) para el caso en que no se ha reducido la eficiencia de mezcla. En particular la diferencia puede ser atribuida a nuestro tratamiento para la mezcla convectiva. Recordemos que el esquema empleado para la convección es la MLT doble difusiva para fluidos con gradientes de composición (Grossman and Taam, 1996).

En la fig. (41) graficamos la energía nuclear liberada por la quema de hidrógeno y helio, y el coeficiente de difusión $D$ para el momento de la gran luminosidad por quema de hidrógeno (punto C de la fig. (28)). Notar que en la fig. (41) superior, los picos de liberación de energía debido a la quema de hidrógeno y de helio están ubicados a una profundidad de 0.008 y $0.03 M_{\odot}$ bajo la superficie, respectivamente. Esto está en buen acuerdo con lo encontrado por Herwig y colaboradores (Herwig et al., 1999a).

El pico de energía de la captura de protones por el ${ }^{12} \mathrm{C}$ ocurre a una temperatura por debajo de $T_{8} \approx 1.26$. Las dos zonas convectivas se encuentran separadas por una región de inestabilidad salt finger. La gran diferencia entre nuestros resultados y los de Herwig (Herwig et al., 1999a), es que en el de aquel, las dos zonas convectivas están conectadas por una zona de OV muy delgada, a través de la cual algunos protones alcanzan las capas más profundas. Por lo que algunos protones son quemados algo más adentro. En contraposición, como nuestros modelos poseen una región de inestabilidad salt finger, evita que esto ocurra. 

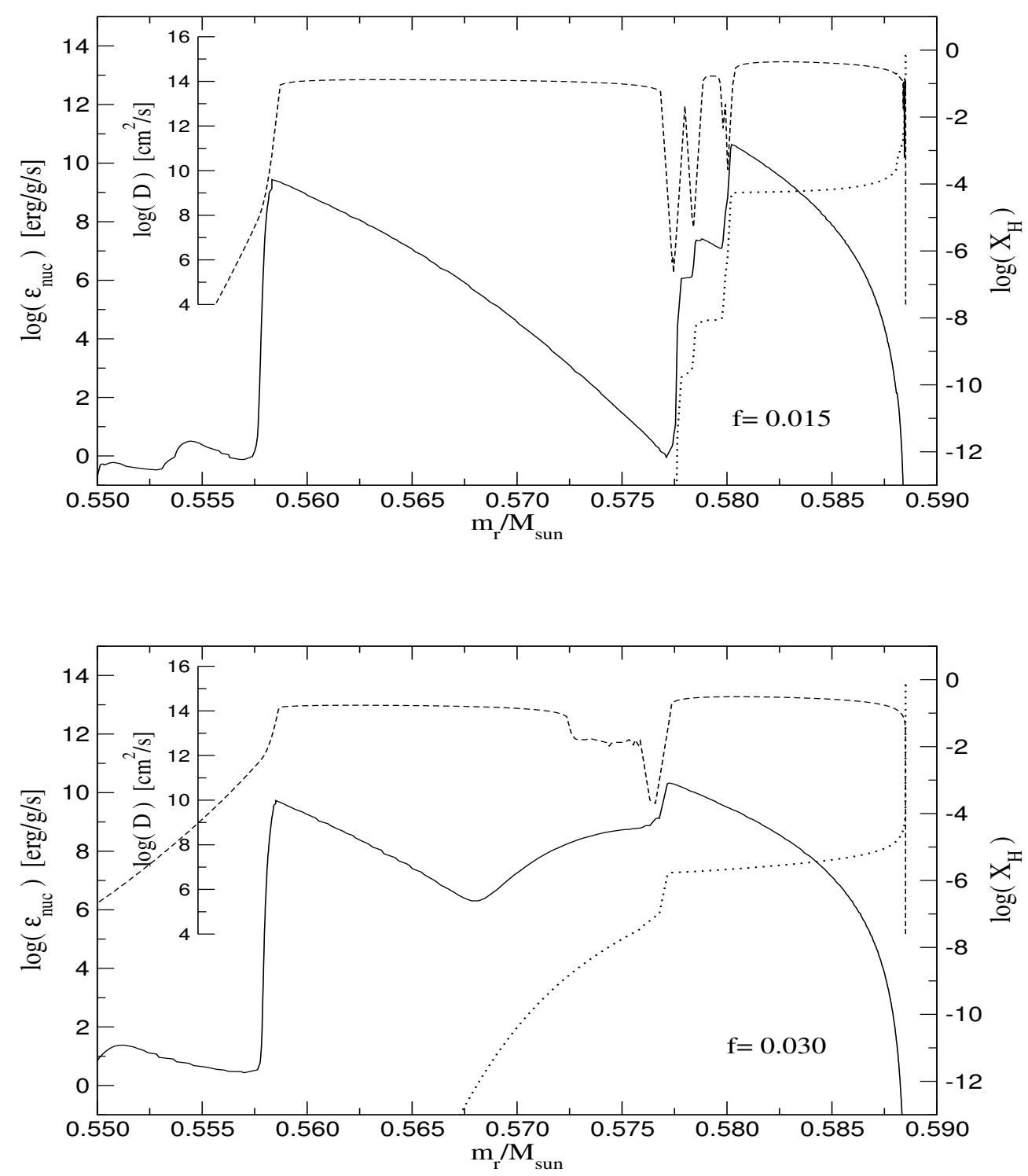

Figura 41: Figura superior: mostramos la liberación de energía nuclear por quema de hidrógeno y helio, y el coeficiente de difusión (línea llena y segmentada, respectivamente, escala izquierda), y perfil de hidrógeno (línea punteada, escala derecha) para el máximo de luminosidad por quema de hidrógeno durante el último pulso térmico - punto $C$ de la fig. (28). Observar los picos de liberación de energía por quema de hidrógeno y helio debajo de la superficie estelar hacia 0.008 y $0.03 M_{\odot}$, respectivamente. Notar la presencia de las dos zonas convectivas (caraterizadas por grandes valores de D) las cuales están separadas por una región de inestabilidad salt finger. El parámetro de OV es $f=0.015$. Figura inferior: al igual que en la superior, pero para un parámetro de $O V$ de $f=0.030$. Las 2 zonas convectivas están conectadas por una zona de $O V$, con la consecuencia de que algunos protones son ingeridos por la zona convectiva del flash de helio. 
En efecto, notar que la región salt finger es lo suficientemente ancha como para que las regiones de OV no superpongan las zonas de inestabilidad convectiva. El resultado neto es que las zonas de quema de hidrógeno y de helio se hallen bien separadas. Para estudiar cuál es la dependencia de este resultado sobre el parámetro de overshoot $f$, hemos recomputado la evolución desde poco antes del último pulso térmico pero considerando un parámetro de eficiencia de mezcla mayor. En este caso se ha tomado un valor de $f=0.030$. Esto podemos verlo en la fig. (41) inferior. Como podemos ver ahora las zonas convectivas se hallan conectadas gracias al OV, lo que tiene como consecuencia que algunos protones sean ingeridos en la zona convectiva del flash de helio. Puesto que los protones son quemados ahora más adentro en la estrella, se espera que esto altere la evolución siguiente. Esto puede observarse en la fig. (42), la cual ilustra la temperatura efectiva como una función de la edad para los estados evolutivos siguientes al último pulso térmico de helio. Notar que si $f=0.030$ la estrella se expande a dimensiones de gigante en una escala temporal mayor a 300 años, en acuerdo a las predicciones de Herwig (Herwig, 2001b). En contraposición al caso de $f=0.015$ esto ocurre en una escala temporal de 20 - 30 años. Como veremos más adelante, un parámetro de OV de $f=0.030$ está en desacuerdo con lo esperado observacionalmente. Se concluye así que la convección GNA provee un tiempo de escala born again marcadamente menor sin invocar ninguna reducción de la eficiencia convectiva.

Finalmente, otra predicción de nuestros cálculos es la aparición de un doble loop en el diagrama H-R. Esto es la estrella incursiona en la región de las gigantes por segunda vez después del último pulso térmico y antes de ingresar a la rama de enfriamiento de las enanas blancas, como fue señalado por Lawlor y MacDonald (Lawlor and MacDonald, 2003), y Herwig (Herwig, 2003) para el caso de baja eficiencia en la mezcla convectiva. En nuestro caso la segunda vuelta hacia le estado de gigante roja lo realiza después de 350 años. 


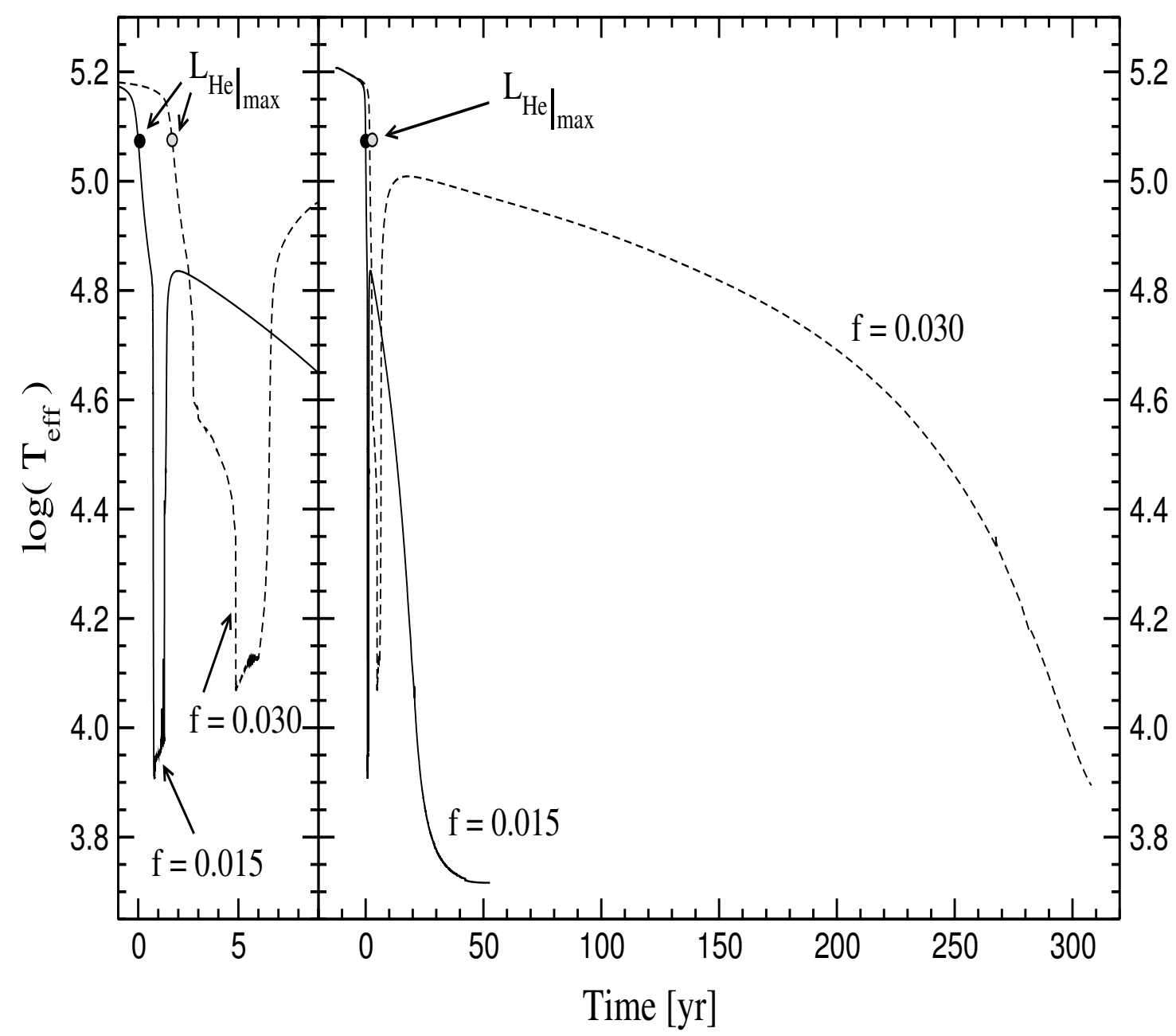

Figura 42: Temperatura efectiva vs. tiempo para los estados evolutivos desde el último pulso térmico de helio y subsiguientes. Las líneas llenas y segmentadas corresponden a valores del parámetro de $O V f$ de 0.015 y 0.030, respectivamente. Para mayor claridad los primeros 9 años de la evolución son expandidos en el gráfico de la izquierda. En cada secuencia el cero en el tiempo corresponde al pico del pulso térmico de helio. Notar que en el caso de mayor eficiencia de $O V(f=0.030)$, la evolución born again es considerablemente mayor a aquella para el caso de $f=0.015$. Gráfico obtenido a partir de nuestros resultados. 


\section{$9 \quad$ Las estrellas PG1159}

\subsection{Generalidades}

El complemento observacional de las estrellas post-AGB deficientes en hidrógeno son las estrellas PG1159 y sus posibles progenitores, las estrellas centrales de las nebulosas planetarias Wolf-Rayet. Las estrellas pre-enanas blancas PG1159 son los objetos más calientes conocidos, cuyo principal exponente es la PG1159-035 (=GW Vir) ${ }^{3}$, la que da nombre a la serie. Análisis espectroscópicos revelan que la mayoría de las estrellas post-AGB están caracterizadas por deficiencia de hidrógeno y abundancias superficiales ricas en helio, carbono y oxígeno. La banda de inestabilidad de las GW Vir está compuesta por estrellas post-AGB deficientes en hidrógeno. Los límites observacionales determinados para la banda de inestabilidad de las variables GW Vir en el diagrama $T_{\text {eff }}-\log g$, son para el borde azul de $180000 \mathrm{~K}$ hasta el borde rojo hacia los $75000 \mathrm{~K}$, la cual es la más fría de las estrellas PG1159 conocidas. Para un modelo con masa $\left(M_{Z A M S}, M_{W D}\right)=\left(2.7 M_{\odot}, 0.5885 M_{\odot}\right)$ se muestra en la fig. (43) la parte de la trayectoria durante la cual la estrella atraviesa el régimen de pre-enana blanca PG1159. Los círculos grises muestran aproximadamente los extremos de la banda de las PG1159, los círculos negros indican los modelos con los tiempos, correspondientes a los perfiles internos de composición química de la fig. (44).

Existe fuerte evidencia observacional que sugiere que las PG1159 son los predecesores directos de la mayoría de las estrellas ricas en helio, las enanas blancas calientes DO, los progenitores inmediatamente anteriores de las enanas blancas DB. La existencia de un nexo evolutivo entre las PG1159 y la mayoría de las DO ha tomado lugar sobre los fundamentos teóricos dados por Unglaub y Bues (Unglaub and Bues, 2000), basados en cálculos de difusión con pérdida de masa para estrellas enanas blancas calientes. En la actualidad se conocen más de treinta PG1159, nueve de ellas se encontraron que eran variables, cinco pertenecen al grupo de las PNNVs (estas son estrellas pre-enanas blancas variables, rodeadas por nebulosas planetarias, y denominadas "Núcleos de Nebulosas Planetarias Variables (PNNV)" ) y cuatro a las estrellas GW Vir (carentes de la nebulosa circundante). Como hemos mencionado las PG1159 son los objetos más calientes conocidos, van desde temperaturas efectivas de $75000 \mathrm{~K}$ (HS0704+6153) hasta $T_{\text {eff }}=180000 \mathrm{~K}$ (RXJ0122.9-7521), lo cual representa un rango bastante amplio en temperatura y la región en el diagrama $\mathrm{H}-\mathrm{R}$ se superpone con la de las enanas blancas DO (Dreizler and Heber, 1998). Las gravedades superficiales también cubren un amplio rango entre $\log g=5.5-8.0$. Estas estrellas tienen típicamente una composición superficial de $\left({ }^{4} \mathrm{He},{ }^{12} \mathrm{C},{ }^{16} \mathrm{O}\right)=(0.33,0.50,0.17)$ por masa (para PG1159-035).

Las estrellas PG1159 proveen un nexo evolutivo entre las estrellas centrales de nebulosas

\footnotetext{
${ }^{3}$ Detectada en el Catálogo de Green de Palomar (Green et al., 1986).
} 


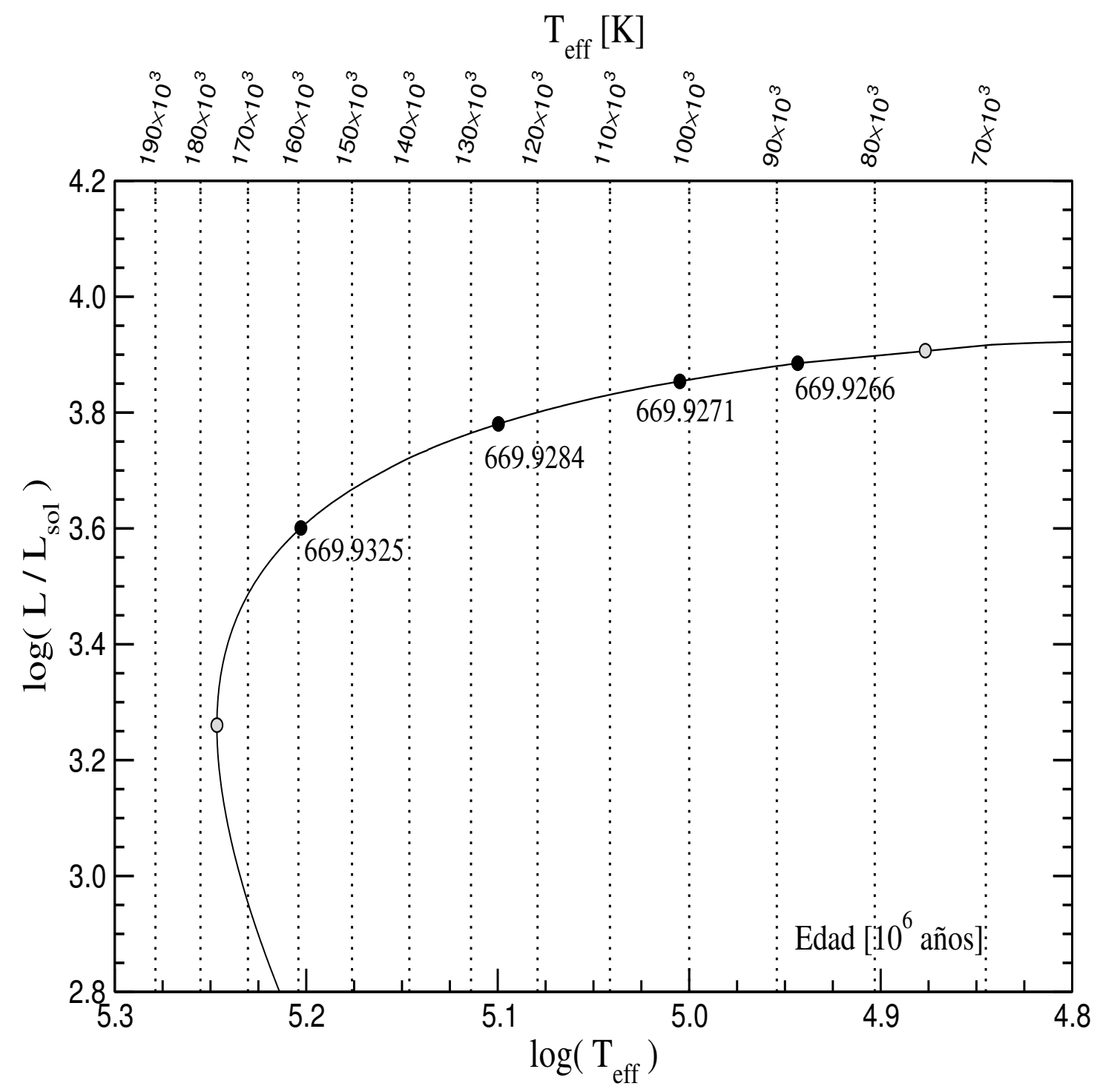

Figura 43: Parte de la trayectoria evolutiva en el diagrama HR de una estrella con una masa $\left(M_{Z A M S}, M_{W D}\right)=\left(2.7 M_{\odot}, 0.5885 M_{\odot}\right)$, en la etapa de estrella pre-enana blanca PG1159. Indicamos con círculos negros los cuatro tiempos correspondientes a la fig. (44). Los círculos grises indican los extremos de la banda de las PG1159. 
planetarias ricas en helio y en carbono (tipo espectral [WC]) y la secuencia de las enanas blancas ricas en helio (DO y DB). Las enanas blancas calientes ricas en helio (DO) se las observan como las sucesoras de las estrellas PG1159. El asentamiento gravitacional de los elementos pesados, probablemente retrasado por la aceleración radiativa, transforma a una estrella PG1159 en una DO, si es que no quedan rastros de hidrógeno en su envoltura. Las estrellas PG1159 también pueden transformarse en enanas blancas DA ricas en hidrógeno.

\subsection{Evolución de las PG1159}

Las abundancias muy elevadas de carbono y oxígeno sobre la superficie de las estrellas PG1159 indican la presencia de material procesado debido a la reacción triple $\alpha$. La abundancia del nitrógeno en este contexto es muy interesante para la evolución de las estrellas PG1159, puesto que la existencia de nitrógeno en un entorno dominado de carbono y oxígeno es difícil de explicar para la evolución nuclear. Todo el nitrógeno es destruido en el proceso triple $\alpha$. Los procesos de mezcla conducen a la destrucción de hidrógeno durante el flash de la capa de helio.

Las abundancias de carbono y oxígeno son de especial interés para la conección evolutiva entre las enanas blancas DO y las estrellas PG1159. Se cree que el asentamiento gravitacional remueve los elementos más pesados dejando una atmósfera rica en helio (asumiendo que la estrella ya está libre de hidrógeno) cuando la estrella evoluciona hacia una estrella DO. La transición parece completarse en la posición de las estrellas PG1159 más frías.

Las abundancias de carbono y oxígeno en las estrellas pulsantes parece ser levemente mayor que en las no-variables en concordancia con las predicciones teóricas que las pulsaciones son conducidas por la ionización cíclica de carbono y oxígeno. Todas las estrellas GW Vir son ricas en nitrógeno, y no se ha detectado nitrógeno en las no-variables. Esta correlación provee la clave para entender el mecanismo de conducción. La abundancia de nitrógeno es también la marcadora de la historia evolutiva de estas estrellas. A fin de tener nitrógeno en un entorno extremadamente rico en carbono y oxígeno, se requieren procesos de mezcla conduciendo al agotamiento de hidrógeno y quema durante un flash en la capa de helio.

En nuestros modelos encontramos que en las capas externas las especies dominantes son ${ }^{4} \mathrm{He},{ }^{12} \mathrm{C}$ y ${ }^{16} \mathrm{O}$ con una abundancia por masa de $\left({ }^{4} \mathrm{He},{ }^{12} \mathrm{C},{ }^{16} \mathrm{O}\right)=(0.306,0.376,0.228)$. También es importante destacar las siguientes abundancias por masa $\left({ }^{13} \mathrm{C},{ }^{14} \mathrm{~N},{ }^{22} \mathrm{Ne}\right)=$ $(0.040,0.012,0.021)$. En la fig. (44) se representan los perfiles internos de ${ }^{4} \mathrm{He},{ }^{12} \mathrm{C},{ }^{13} \mathrm{C}$, ${ }^{14} \mathrm{~N} \mathrm{y}{ }^{16} \mathrm{O}$ para cuatro instantes evolutivos distintos.

$\mathrm{El}^{13} \mathrm{C}$ y ${ }^{14} \mathrm{~N}$ se encuentran presentes desde las capas más exteriores hasta adentro en la base de la primer capa convectiva superior en una profundidad debajo de la superficie estelar de $0.01 M_{\odot}$. En las capas más profundas ellos fueron quemados mediante capturas $\alpha$. La 

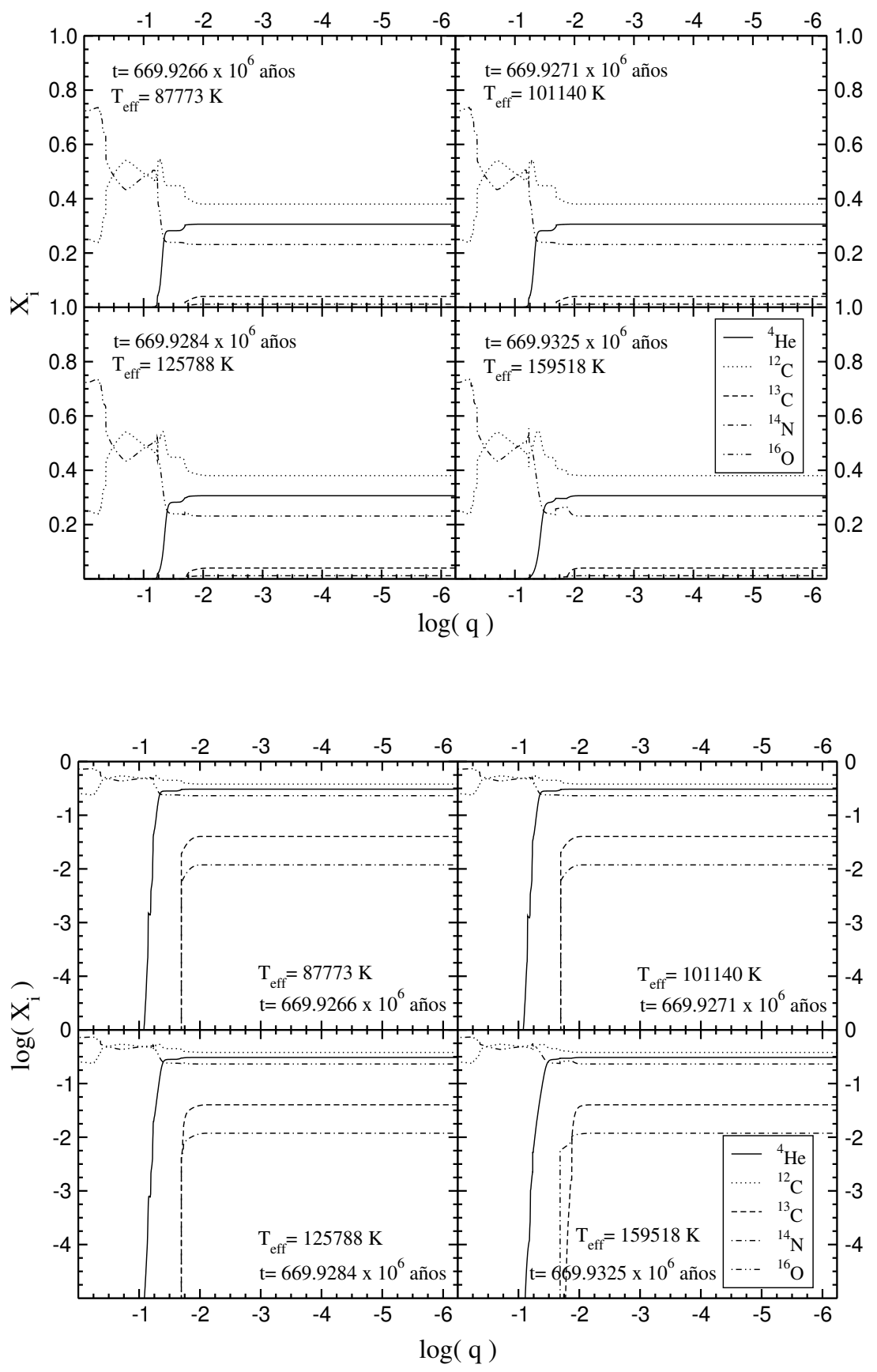

Figura 44: Figura superior: abundancias químicas internas de cuatro instantes evolutivos distintos para un modelo con una masa de $\left(M_{Z A M S}, M_{W D}\right)=\left(2.7 M_{\odot}, 0.5885 M_{\odot}\right)$, en el régimen de preenana blanca PG1159. Se representan las abundancias de ${ }^{4} \mathrm{He},{ }^{12} \mathrm{C},{ }^{13} \mathrm{C},{ }^{14} \mathrm{~N}$ y ${ }^{16} \mathrm{O}$ en función de la fracción de masa q para el remanente de $0.5885 M_{\odot}$. Notar que en la figura para $t=669.9325 \times$ $10^{6}$ años aparece un aumento para el oxígeno como resultado de la captura de partículas $\alpha$ por el ${ }^{13}$ C. Figura inferior: idem fig. superior pero con las abundancias dadas en escala logarítmica. 
gran fracción de masa de oxígeno es un indicio de la presencia de OV difusivo durante la fase AGB de los pulsos térmicos esto está en buen acuerdo con las predicciones de los cálculos de la evolución born again realizadas por Herwig y colaboradores (Herwig et al., 1999a). Las composiciones superficiales finales de nuestros modelos concuerdan con las composiciones observadas en las estrellas más calientes post-AGB deficientes en hidrógeno como las estrellas PG1159 y las estrellas centrales de las nebulosas planetarias de tipo espectral WC -(Werner, 2001); (Dreizler and Heber, 1998); (Koesterke and Hamann, 1997). Cabe destacar el acuerdo entre la abundancia de ${ }^{14} \mathrm{~N}$ de nuestros cálculos y el detectado por Dreizler y Heber (Dreizler and Heber, 1998) en cinco de nueve estrellas PG1159 que soportan la idea de que estas estrellas podrían ser las descendientes AGB que han experimentado un episodio born again. En efecto, la mezcla y quema de protones en la zona convectiva del flash de helio es necesaria para crear ${ }^{14} \mathrm{~N}$ en un medio rico de carbono y oxígeno. Sin embargo, encontramos que en el caso de una gran eficiencia de OV $(f=0.03)$, la fracción superficial de masa de ${ }^{14} \mathrm{~N}$ es reducida a $10^{-6}$ y las principales especies superficiales cambian a $\left({ }^{4} \mathrm{He},{ }^{12} \mathrm{C},{ }^{16} \mathrm{O}\right)=$ $(0.27,0.42,0.277)$ con una abundancia demaciado grande de oxígeno para que sea compatible con las observaciones de las estrellas post-AGB deficientes en hidrógeno. Esto sería un indicio de que el parámetro de OV en la zona convectiva del flash de helio no podría ser tan grande como $f=0.03$. La abundancia superficial encontrada extremadamente baja de ${ }^{14} \mathrm{~N}$ está en desacuerdo con lo detectado sobre este elemento, el cual es abundante en algunas estrellas PG1159 (Dreizler and Heber, 1998).

Notemos que la evolución a través del dominio de las estrellas PG1159, muestra cambios en la composición química que ocurre debido a la quema nuclear. Esto puede verse en la fig. (44). En la misma, para $t=669.9325 \times 10^{6}$ años puede verse que para el ${ }^{16} \mathrm{O}$ hacia $\approx 10^{-2} M_{\odot}$ debajo de la superficie estelar aparece un aumento, debido a la quema del ${ }^{13} \mathrm{C}$ mediante la reacción ${ }^{13} \mathrm{C}(\alpha, n){ }^{16} \mathrm{O}$. Antes de que el remanente alcance el máximo de temperatura efectiva en el diagrama $\mathrm{H}-\mathrm{R}$, la temperatura de la base de la distribución de ${ }^{13} \mathrm{C}$ excede a $85 \times 10^{6} \mathrm{~K}$, la cual es lo suficientemente alta como para que opere la reacción ${ }^{13} \mathrm{C}(\alpha, n){ }^{16} \mathrm{O}$. Durante el estado de PG1159 el contenido de helio en la estrella se reduce de $8.5 \times 10^{-3}$ a $5.2 \times 10^{-3} M_{\odot}$, como resultado de la quema de helio. Esta abundancia representa la cantidad con la que la estrella entra al dominio de las enanas blancas después de que se ha extinguido la quema de helio. 


\section{Aspectos generales de las enanas blancas}

\section{1 ¿Cuáles estrellas se convierten en enanas blancas?}

Como es sabido la máxima masa de una estrella de carbono-oxígeno que puede convertirse en enana blanca está dada por la masa límite de Chandrasekhar -ver Apéndice A, sec. (13.7.3)- y es de $\sim 1.4 M_{\odot}$, las estrellas de baja masa descriptas en la sec. (4.5) no tienen dificultad en alcanzar este estado final. Las estrellas con masas mayores sobre la secuencia principal también se las arreglan para alcanzar este estado. Una evidencia directa es a través de la observación de los cúmulos estelares. Es conocido entre los astrofísicos que estos grupos estelares en donde sus componentes poseen diferentes masas, se forman al mismo tiempo. Debido a que la luminosidad sobre la secuencia principal aumenta mucho más rápido que la masa ${ }^{4}$ una estrella masiva necesita un tiempo más corto para consumir su combustible nuclear y comenzar la evolución hacia la rama gigante, que una de menor masa.

Las secuencias principales de los cúmulos queman su núcleo más rápidamente desde arriba de la secuencia y hacia abajo. Las estrellas más masivas del cúmulo que todavía están en la secuencia principal dan un indicio de la edad del cúmulo. Este punto es conocido como turnoff point. Por ejemplo en el cúmulo de las Hyades las estrellas con masas $M \approx 2 M_{\odot}$ están todavía en la secuencia principal jsin embargo el cúmulo posee alrededor de una docena de enanas blancas! Estas enanas blancas tienen masas típicas de $0.6 M_{\odot}$, pero sobre la secuencia principal las estrellas debieron haber sido más masivas que las del turn-off para que hayan evolucionado a enana blanca.

La solución a este enigma, es la pérdida de masa durante la evolución. Aún en estrellas normales como nuestro sol, hay evidencia de una pérdida de masa continua (viento solar); en las gigantes rojas la pérdida de masa observada es varios órdenes de magnitud mayor (Blöcker, 1995b). Existirá entonces, para las estrellas sobre la secuencia principal, una masa crítica por debajo de la cual las estrellas evolucionarán a enanas blancas. Es sabido que el remanente de una estrella masiva es una enana blanca más masiva y viceversa.

En conclusión las estrellas moderadamente masivas, posiblemente hasta $8 M_{\odot}$, terminarán como enanas blancas. Comparando este límite con la distribución de masas sobre la secuencia principal esto determina que el $90 \%$ de todas las estrellas se convierten en enanas blancas.

\subsection{Aspectos observacionales}

De los datos de la distribución de energía, espectros ópticos y ultravioletas, se sabe que la temperatura efectiva $\left(T_{\text {eff }}\right)$ de las enanas blancas es de $T_{\text {eff }} \sim 150000 \mathrm{~K}$ para los objetos

\footnotetext{
${ }^{4} L / L_{\odot} \propto\left[M / M_{\odot}\right]^{\eta}$ en un rango de $1-10 M_{\odot}, \eta=3.88$; para $1-40 M_{\odot}, \eta=3.35$; para todo el rango de
} masas es $\eta=3.2$ 
más calientes, hasta $T_{\text {eff }} \sim 4000 \mathrm{~K}$ para los más fríos. Los análisis espectroscópicos también proporcionan la gravedad superficial $(\log g)$, debido a que la intensidad y el ancho de las líneas espectrales son sensibles a la densidad del gas en la atmósfera, la cual es función de la gravedad superficial. Sin embargo, para las enanas blancas más frías toda información deducible a partir de los rasgos espectrales desaparece y no pueden obtenerse determinaciones espectroscópicas de $\log g$. En este caso es necesario, a partir de mediciones de la paralaje, determinar el radio estelar y la masa (a través de la relación masa-radio), y finalmente deducir la gravedad. El valor promedio para enanas blancas es de $\log g \sim 8$ (nótese que el valor solar es de $\log g \sim 4.4)$. El intervalo en luminosidad $(L)$ cubierto por las enanas blancas es muy amplio, reflejando el enorme rango en $T_{\text {eff }}$. Así, las enanas blancas más calientes alcanzan un valor de $L \sim 10^{2}-10^{3} L_{\odot}$, mientras que las más frías tienen un valor de $L \sim 10^{-4.7} L_{\odot}$. La magnitud visual aparente $(V)$ de las enanas blancas es, en promedio, de $V \sim 15.5^{m}$.

Por ser objetos muy débiles deben detectarse en forma indirecta, como por ejemplo, mediante perturbaciones gravitatorias sobre estrellas visibles, a través de los movimientos propios y sólo para objetos próximos al Sol. De estos objetos, las candidatos a ser enanas blancas son aquellas estrellas azules o muy azules, que las distinguen de estrellas de secuencia principal o próximas a ella; la confirmación proviene por medio de análisis espectroscópicos. Es un hecho bien establecido que la distribución de enanas blancas en las vecindades del Sol corresponde a una población de disco (Población I), con una escala de altura de $250-300$ pc. La densidad espacial es del orden de $0.005 \mathrm{pc}^{-3}$ para magnitudes bolométricas $M_{\mathrm{bol}}<15^{m}$.

\subsection{Aspectos estadísticos}

Los antecesores directos de la mayor parte de las enanas blancas son núcleos de nebulosas planetarias, formadas estas últimas a partir de estrellas de masa baja e intermedia de la secuencia principal. Se estima que estrellas que comienzan su evolución en la secuencia principal con una masa estelar $\lesssim 8 M_{\odot}$ (o la mayoría de ellas) terminan su vida como enanas blancas. La mayoría de las enanas blancas ha quemado hidrógeno y helio en su interior en su historia previa, por lo cual gran parte de su estructura interna está conformada por un núcleo de carbono y oxígeno (el cual contiene el $99 \%$ de la masa total de la estrella).

La distribución observada de masas de enanas blancas aisladas tiene un pico muy pronunciado alrededor de un valor de $M_{*} \sim 0.59 M_{\odot}$. Si bien el rango observado de masas es de $0.3 M_{\odot} \lesssim M_{*} \lesssim 1.2 M_{\odot}$ (donde las colas de la distribución podrían deberse a la formación de enanas blancas producto de la evolución binaria), la mayor parte de las enanas blancas tienen masas muy próximas a la masa promedio, con una dipersión muy pequeña de $\sim 0.13 M_{\odot}$. Claramente los procesos de pérdida de masa experimentado por los progenitores (los cuales 
tienen inicialmente un rango amplio de masas) son tal que el resultado final es, en todos los casos, la aparición de enanas blancas con masas muy similares. Así, a pesar de sus diversos orígenes y luminosidades, las enanas blancas forman una clase marcadamente homogénea de estrellas.

Existe evidencia de que pequeñas cantidades de helio e hidrógeno permanecen en la estrella luego de que los procesos de pérdida de masa han actuado. Tomando en cuenta los procesos termonucleares previos y la acción de la sedimentación gravitacional, la estructura de una enana blanca típica debe ser (esperable desde la teoría) un objeto estratificado formado por un núcleo de carbono-oxígeno rodeado por una fina envoltura rica en helio, rodeada a su vez por una capa rica en hidrógeno. La densidad media debe ser del orden de $10^{6} \mathrm{~g} \mathrm{~cm}^{-3}$. El espesor de las envolturas de hidrógeno y helio depende de los detalles de la evolución previa, y no son cantidades conocidas observacionalmente (excepto por determinaciones astrosismológicas). A partir de consideraciones teóricas, sin embargo, se cree que los valores máximos de la masa de helio e hidrógeno que puede sobrevivir a la combustión nuclear en la etapa de nebulosa planetaria son de $\sim 10^{-2} M_{*} \mathrm{y} \sim 10^{-4} M_{*}$, respectivamente. Si bien estas capas son muy delgadas, ellas son extremadamente opacas a la radiación, y actúan como reguladoras del flujo de energía que escapa al espacio. Por lo tanto el valor preciso del espesor de tales envolturas es de fundamental importancia en la evolución de las enanas blancas.

\subsection{Clasificación espectral}

El esquema más común de clasificación espectral es reproducido en la tabla [(3), sec. (10.4)] (Hansen and Kawaler, 1994). La letra 'D' proviene de dwarf (enana). Si se puede hacer una estimación de la temperatura, entonces a la designación se le agrega un dígito indicador de temperatura $\left(\begin{array}{l}0 \\ \text {... }\end{array}\right)$. En algunos casos las designaciones pueden ser combinadas, por ejemplo "DZA" que indica que es un espectro dominado por líneas de metales pero mostrando también líneas débiles de hidrógeno (cuando hablamos de "metales" nos referimos a elementos más allá del helio). Los espectros de las enanas blancas se pueden ubicar en dos grupos bien definidos: aquellos con atmósferas dominadas por el hidrógeno, DA, y las atmósferas dominadas por el helio (DB, DC, DO, DZ, DQ) o no-DA. Los distintos tipos espectrales se ubican en el gráfico de la fig. (45).

La ubicación de las enanas blancas según los rangos de temperatura es el siguiente:

DO: esta clase está caracterizada por las atmósferas dominadas por el helio en un rango de 45000 a $100000 \mathrm{~K}$. El hidrógeno está presente con una abundancia numérica $\gtrsim 1 \%$.

DA: esta clase es por lejos la mayor y está observada sobre casi el rango completo de temperaturas donde las líneas de Balmer son visibles, desde los 6000 hasta los $\sim 70000 \mathrm{~K}$. 
Las líneas de Balmer podrían ser visibles aún a temperaturas mayores, pero no como DA. Las DAO $\left(T_{\text {eff }} \sim 60000 \mathrm{~K}\right)$ tienen abundancias de helio del orden de $1 \%$.

DB: esta clase muestra sólo líneas de HeI y es observada entre 12000 y $30000 \mathrm{~K}$, pero está ausente en el rango de 30000 y 45000K. Hay observaciones de DB que muestran líneas de hidrógeno débiles (DBA) con abundancias del orden de $10^{-4}$.

DC: históricamente las más frías con un espectro rico en helio, debajo del rango de las DB $(<12000 \mathrm{~K})$ fueron clasificadas como DC.

DQ: muestran líneas resonantes del CI. Las abundancias típicas de carbono en estos objetos se encuentran entre $10^{-7}$ a $10^{-3}$. Se cree que la presencia de ${ }^{12} \mathrm{C}$ en la superficie es de origen interno y no interestelar. En efecto gracias al asentamiento gravitacional que actúa en las capas exteriores de las enanas blancas, se espera que una capa delgada de helio flote en la parte superior del siguiente elemento pesado (carbono), fabricado por la quema de helio en etapas evolutivas previas. Si la zona convectiva en la capa de helio alcanza la parte inferior, de la cola de la distribución de carbono, entonces podría llevar rastros de carbono hacia la superficie, sin transformar las capas superiores casi en carbono puro. Los cálculos detallados predicen un máximo de abundancia de carbono en un rango entre los $11000 \mathrm{y}$ $12000 \mathrm{~K}$ debido a un efecto combinado de las extensiones de la zona convectivas y la presión de radiación.

DZ: son las estrellas no-DA más frías. Gracias a las observaciones del satélite IUE algunas de estas revelaron fuertes características de Mg, Fe y Si en el espectro UV.

\begin{tabular}{|cl|}
\hline \multicolumn{2}{|c|}{ Tabla 3: Clasificación espectral de estrellas enanas blancas. } \\
\hline \hline Tipo Espectral & Características \\
\hline DA & con líneas de H, sin HeI ni metales presentes \\
DB & con líneas de HeI, sin H ni metales presentes \\
DC & espectro contínuo sin líneas distinguibles \\
DO & con líneas intensas HeII; puede haber HeI o H \\
DZ & con líneas de metales, sin H ni He \\
DQ & con líneas de C \\
\hline \hline
\end{tabular}

Observaciones espectroscópicas y fotométricas permiten concluir que la mayoría de las enanas blancas poseen atmósferas de hidrógeno casi puro; usualmente se las denomina enanas blancas DA. Sin embargo, alrededor de un $25 \%$ de las enanas blancas conocidas no poseen tal envoltura de hidrógeno, y exhiben una atmósfera rica en helio; a estas se las cononoce como enanas blancas DB. El resto de las enanas blancas son estrellas con atmósferas híbridas o con abundancias químicas peculiares. 


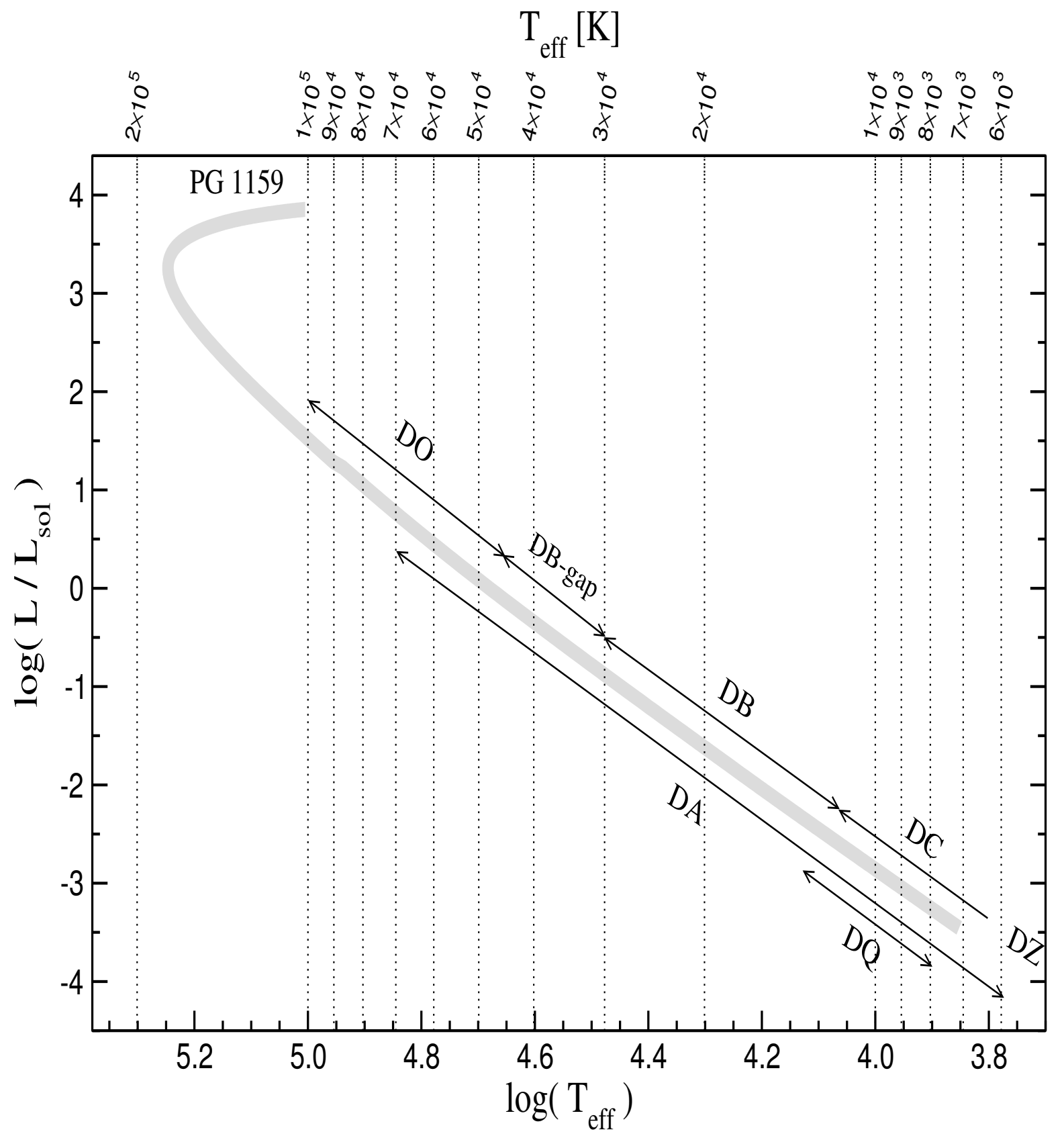

Figura 45: Diagrama de Hertzsprung-Russell esquemático ilustrando la localización de varias clases de estrellas enanas blancas. La banda gris representa la curva de enfriamiento de las enanas blancas. Sobre la misma se indica los distintos tipos espectrales de enanas blancas, se muestra también la brecha en donde están ausentes las DB y las pre-enanas blanca PG1159. 
Existen numerosas evidencias observacionales de que alguna clase de evolución espectral tiene lugar a lo largo de la evolución de las enanas blancas, ya que algunas DA se tornan DB y viceversa en algunas etapas del enfriamiento. Tal es así que el cociente entre enanas blancas DA y DB es diferente para distintas temperaturas efectivas. En particular, es evidente la existencia del llamado gap de las DB, situado a $45000 \gtrsim T_{\text {eff }} \gtrsim 30000 \mathrm{~K}$, en el cual no se han podido encontrar enanas blancas tipo DB. También es conocido otro intervalo en $T_{\text {eff }}$, más estrecho, situado entre los $6000 \gtrsim T_{\text {eff }} \gtrsim 5000 \mathrm{~K}$, en el cual tampoco se encuentran objetos tipo DB. Si bien no hay explicaciones completamente satisfactorias para estas anomalías en la distribución de enanas blancas con atmósferas ricas en helio, se sospecha que algún tipo de interrelación entre procesos de difusión química y convección debe actuar para que algunas estrellas cambien su composición química superficial.

\subsection{Aspectos evolutivos}

La mayoría de las enanas blancas nacen (de acuerdo a su condición previa de núcleo de nebulosa planetaria) como objetos compactos extremadamente calientes los cuales comienzan a enfriarse, con sus fuentes de energía nuclear casi agotadas; la energía gravitacional no puede ser liberada eficientemente debido a que la presión de los electrones degenerados impide cualquier contracción mayor adicional: la estrella está obligada a evolucionar a radio casi constante, siendo el decrecimiento de la energía interna almacenada en los iones su fuente de luminosidad. Entonces, el problema de la evolución de una enana blanca puede representarse básicamente como un problema de enfriamiento.

Una propiedad inherente a los electrones degenerados es su alta conductividad del calor, a raíz de lo cual el núcleo de las enanas blancas es casi isotermo. Su temperatura varía entre $\sim 2 . \times 10^{7} \mathrm{~K}$ y $\sim 5 . \times 10^{6} \mathrm{~K}$ para enanas blancas en el rango de $T_{\text {eff }}$ de $16000-8000 \mathrm{~K}$. La gran caída en la temperatura yendo del centro a la superficie, tiene lugar principalmente en la envoltura estelar, que actúa como aislante. Este enorme gradiente de temperatura da lugar a la aparición de zonas convectivas superficiales en las enanas blancas más frías. Estas zonas convectivas, cuando existen, regulan el flujo emergente de energía hacia el exterior. También juegan un papel clave en la tasa de enfriamiento de la estrella cuando la convección alcanza el borde externo del núcleo degenerado, acoplándolo con la superficie y así aumentando el flujo de energía a través de la envoltura (en comparación con el caso en que el transporte es radiativo).

Como se mencionó, los responsables de proveer la energía térmica que luego resulta en la luminosidad de la enana blanca son los iones (no degenerados). De hecho, la estrella brilla principalmente a expensas del calor acumulado en los iones durante su historia evolutiva previa (los electrones no contribuyen significativamente). A medida que la energía térmica 
es gradualmente perdida por la estrella en forma de radiación, la energía cinética de los iones disminuye y el gas de iones pasa de un estado gaseoso a un estado fluido y luego a un estado sólido. El pasaje del estado fluido a sólido (cristalización) trae aparejada una liberación de calor latente, capaz de frenar considerablemente el enfriamiento de la estrella. Finalmente la reserva de energía térmica se agota y la estrella, ahora casi completamente cristalizada, pasa a la invisibilidad.

Una interesante propiedad de las enanas blancas es que, como mencionamos antes, algunas de ellas experimentan inestabilidades pulsacionales. En efecto, a lo largo de su enfriamiento, atraviesan dos bandas de inestabilidad: la de las DAV y DBV -ver sec. (10.6). Las inestabilidades se manifiestan en términos de modos $g$ no-radiales con períodos de oscilacion comprendidos entre 100 y 1200 segundos. La estructura del espectro de períodos es fuertemente dependiente de la estructura interna de estas estrellas (en particular de la estructura química interna). De esta manera estas estrellas ofrecen la posibilidad de sondear su interior estudiando sus propiedades vibracionales. De hecho, los estudios de pulsaciones de enanas blancas constituyen el mejor ejemplo de aplicación de la astrosismología -ver (Córsico, 2003).

\subsection{Enanas blancas variables: bandas de inestabilidad}

Existen varias clases de enanas blancas pulsantes, las cuales definen bandas de inestabilidad bien localizadas en el diagrama $\mathrm{H}-\mathrm{R}$. Una lista completa conteniendo las propiedades de las distintas clases de enanas blancas pulsantes puede encontrarse en el trabajo de Bradley (Bradley, 2000). En la fig. (46) se ubican los distintos tipos de estrellas pulsantes, el mismo es una adaptación de Christensen-Dalsgaard (Christensen-Dalsgaard, 1998).

\subsubsection{Estrellas pre-enanas blancas variables}

Están ubicadas en la región del diagrama $\mathrm{H}-\mathrm{R}$ donde la trayectoria las estrellas post-AGB se une a las secuencias de enfriamiento de las enanas blancas. Estas estrellas tienen muy altas temperaturas efectivas, con $7 \times 10^{4} \mathrm{~K} \lesssim T_{\text {eff }} \lesssim 1.7 \times 10^{5} \mathrm{~K}$. Espectroscópicamente muestran fuertes deficiencias en hidrógeno y rasgos pronunciados de carbono, helio y oxígeno [(Werner, 1992); (Dreizler et al., 1995)]. Muestran periodicidades fotométricas con períodos entre 7 y 30 minutos, y se cree que son debidas a modos $g$ (gravedad) de bajo grado armónico $\ell$ y alto orden radial $k$. Aquellas pre-enanas blancas variables las cuales están rodeadas por nebulosas planetarias se denominan en la literatura como núcleos de nebulosas planetarias variables (PNNV), y las que carecen de cualquier rastro de nebulosa son llamadas variables PG1159 $(\mathrm{GW} \text { Vir })^{5}$. Los períodos de pulsación de las PNNVs son más largos que los correspondientes

\footnotetext{
${ }^{5}$ En la literatura es también muy común denominarlas DOV.
} 


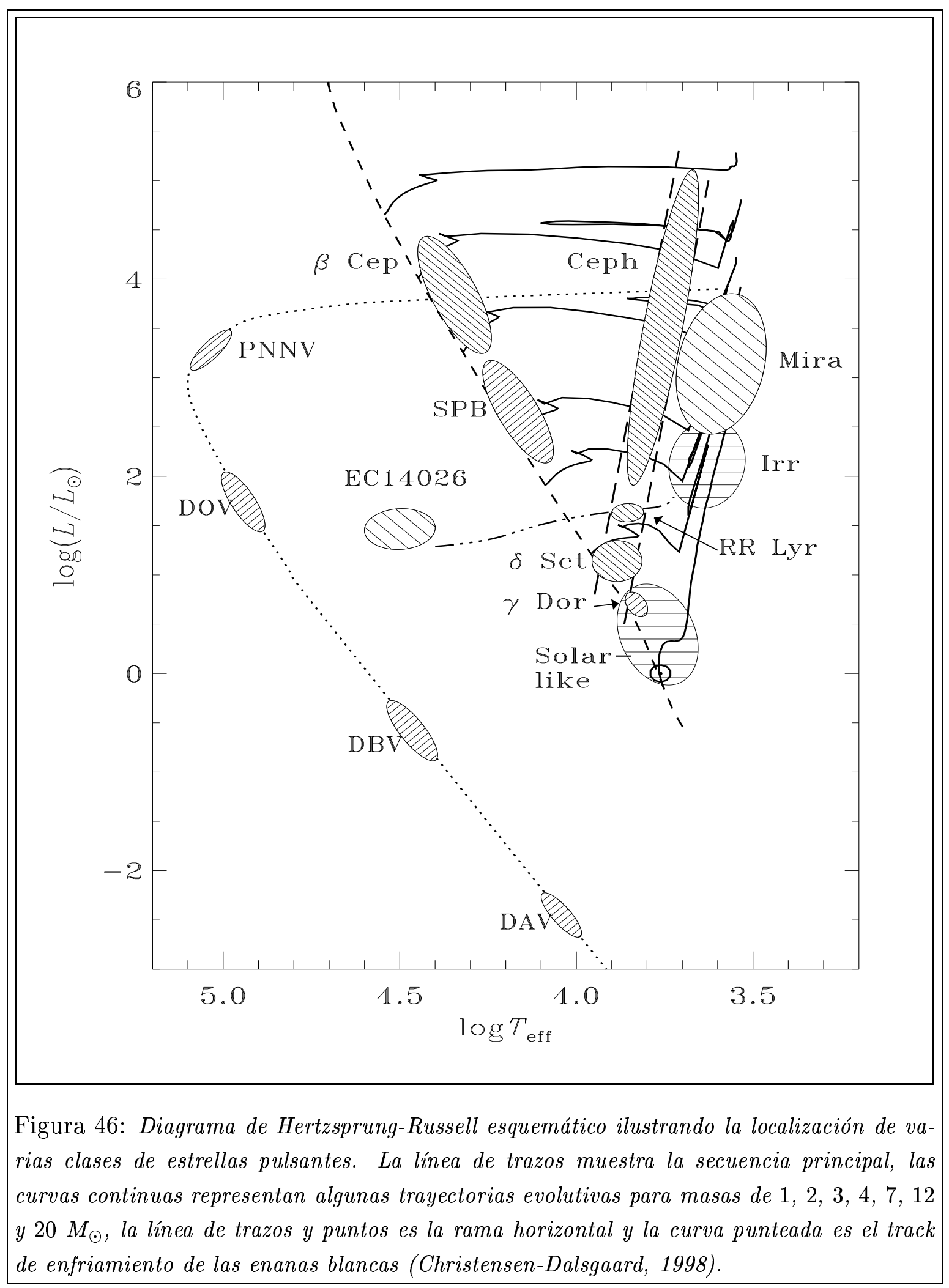


a las PG1159, indicando que las PNNVs tienen mayores radios. Desde el punto de vista evolutivo, las PG1159 son consideradas como objetos que están entrando en las primeras etapas de enfriamiento de la trayectoria de enanas blancas, mientras que, en contraste, las PNNVs están todavía desplazándose hacia altas temperaturas a luminosidad constante. Se conocen nueve miembros de la categoría PNNV. Por otra parte sólo cinco miembros de la clase PG1159 son conocidos hasta el momento. Entre ellas hay una PG1159 "híbrida", denominada HS2324+3944, la cual muestra hidrógeno en su espectro (Silvotti et al., 1999). Es notable que, si bien la región del diagrama H-R donde las PG1159 pulsan está bien determinada, existen entre las variables objetos no-variables: la banda de inestabilidad no es "pura" (Werner, 1993). En el caso de las PG1159, las que pulsan se pueden distinguir de las no-variables por el hecho de que muestran nitrógeno en sus espectros (Dreizler and Heber, 1998).

\subsubsection{Estrellas variables DBV (o V4777 Her)}

Estas enanas blancas variables están confinadas a un rango de $21500 \mathrm{~K} \lesssim T_{\text {eff }} \lesssim 24000 \mathrm{~K}$, con luminosidades $\sim 10^{-1.3} L_{\odot}$. Esta clase de variables es la única cuya existencia fue predicha (Winget et al., 1982b) antes de su descubrimiento, por Winget y colaboradores (Winget et al., 1982a). Los períodos encontrados están en el rango de 140 a 1000 segundos. Actualmente se conocen unas ocho estrellas miembros de esta clase, la mayoría de las cuales muestran espectros de pulsación complejos, con muchas frecuencias presentes. La DBV más estudiada (la primera en descubrirse) es GD358, la cual es al mismo tiempo la más brillante del grupo. La banda de inestabilidad de las DBV tampoco es pura: existe entre un $25 \%$ y un $50 \%$ de estrellas DB no pulsantes en el dominio de inestabilidad, dependiendo de que sus parámetros atmosféricos sean derivados a partir de atmósferas con helio puro o conteniendo pequeñas cantidades de hidrógeno (Beauchamp et al., 1999).

\subsubsection{Estrellas variables DAV (o ZZ Ceti)}

Se conocen 31 miembros de esta clase. En contraste con el caso de las pre-enanas blancas y las DBV, la banda de inestabilidad de las DAV es pura: no hay estrellas DA no-variables en el dominio del diagrama $\mathrm{H}-\mathrm{R}$ donde las DAV están localizadas, en el rango de temperaturas efectivas $12500 \mathrm{~K} \gtrsim T_{\text {eff }} \gtrsim 10700 \mathrm{~K}$. Estudios espectroscópicos de DAV muestran un continuo de cuerpo negro con líneas de absorción de hidrógeno superpuestas, sin señales de helio o metales presentes (Koester \& Chanmugam 1990). Las variabilidades fotométricas de las DAV son multiperiódicas, con períodos entre los 100 y 1200 segundos y amplitudes hasta $0.3^{m}$. En muchos casos las frecuencias están divididas en multipletes, debido a rotación lenta. 
Las observaciones de DAV sugieren que ellas tienden a acumularse en dos grupos principales (Unno et al., 1989): un grupo de estrellas calientes que exhiben un pequeño número de períodos cortos y estables (cambios de amplitud muy pequeños), y otro grupo de estrellas más frías con muchos más períodos presentes pero mostrando fuertes efectos no-lineales y variaciones de amplitud.

Un aspecto importante relacionado con la localización del borde azul de la banda de inestabilidad de las DAV es el de la influencia de la eficiencia convectiva utilizada en la construcción de los modelos en equilibrio. Aparentemente sólo zonas de convección muy extendidas, tratadas con la MLT, dan lugar a bordes azules compatibles con los datos observacionales (Bradley and Winget, 1994a). Las prescripciones ML2 o ML3 -ver (Tassoul et al., 1990)- de la MLT son las que proporcionan el mejor acuerdo con la observación. 


\section{Evolución de las enanas blancas}

Una vez que la quema de helio se ha extinguido para $T_{\text {eff }} \approx 100000 \mathrm{~K}$ después de $10^{5}$ años de evolución desde el último pulso térmico, el remanente se posiciona sobre la rama de enfriamiento de las enanas blancas.

En la fig. (47) se muestra el diagrama $\mathrm{H}-\mathrm{R}$ en donde se representan los puntos correspondientes a los modelos de las figs. (48), (49), (51) y (52). En la misma, la edad está indicada en millones de años y la temperatura efectiva en grados kelvin.

A medida que el remanente evoluciona, modifica la distribución de abundancias debido a procesos de difusión que actúan durante la fase de enfriamiento de la enana blanca. Esto puede verse en las figs. (48), (49), (51) y (52), donde se ilustran las abundancias por masa de ${ }^{4} \mathrm{He},{ }^{12} \mathrm{C},{ }^{13} \mathrm{C},{ }^{14} \mathrm{~N}$ y ${ }^{16} \mathrm{O}$ para un remanente de $0.5885 M_{\odot}$ como función de la fracción de masa, caracterizadas por valores de edad y temperatura efectiva.

\subsection{Primeras etapas de la rama de enfriamiento}

La fig. (48) muestra la estratificación al comienzo de la rama de enfriamiento. Para $t=$ $669.940 \times 10^{6}$ años el modelo se encuentra en el extremo más al azul con una $T_{\text {eff }} \approx 176500 \mathrm{~K}$ y a altas luminosidades.

En los tres modelos siguientes de la figura el remanente comienza a disminuir en luminosidad y a enfriarse. En las capas más exteriores, la composición química corresponde a aquella con la cual el remanente emergió de los eventos de mezcla y quema del último pulso térmico de helio y siguiente episodio de born again.

Rápidamente, el asentamiento gravitacional hace que el helio flote hacia la superficie y los elementos más pesados se desplacen hacia abajo en la envoltura. Este efecto comienza a notarse en la figura con $t=670.089 \times 10^{6}$ años.

\subsection{Enanas blancas DO}

Conforme se sigue enfriando el remanente, observamos como el helio aumenta y los elementos pesados disminuyen en la parte exterior a causa de la difusión -ver fig. (49). En este momento el remanente se halla en el dominio de las enanas blancas DO. El rango de temperatura para estos objetos se encuentra entre los 45000 y $100000 \mathrm{~K}$.

Vemos como se va construyendo para la abundancia de ${ }^{4} \mathrm{He}$ un perfil de capa doble. Esta estructura química de doble capa es desarrollada gracias a la difusión de los elementos químicos. 


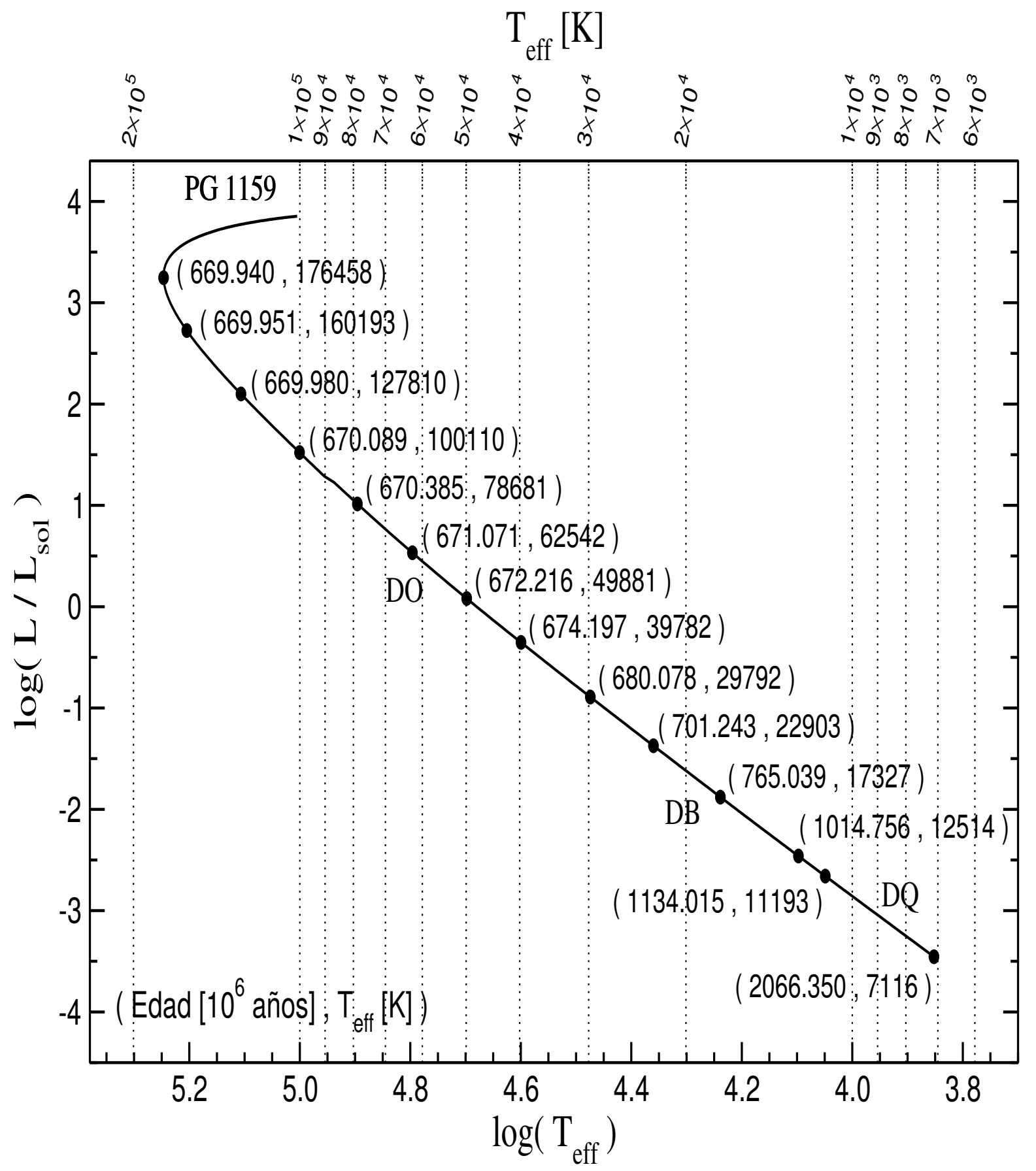

Figura 47: Diagrama $H-R$ de la rama de enfriamiento para el remanente de $0.5885 M_{\odot}$, los círculos negros representan los distintos modelos de las fig. (48), (49), (51) y (52). Las edades están en millones de años y las temperaturas efectivas en grados kelvin. Obtenido a partir de nuestros resultados. 

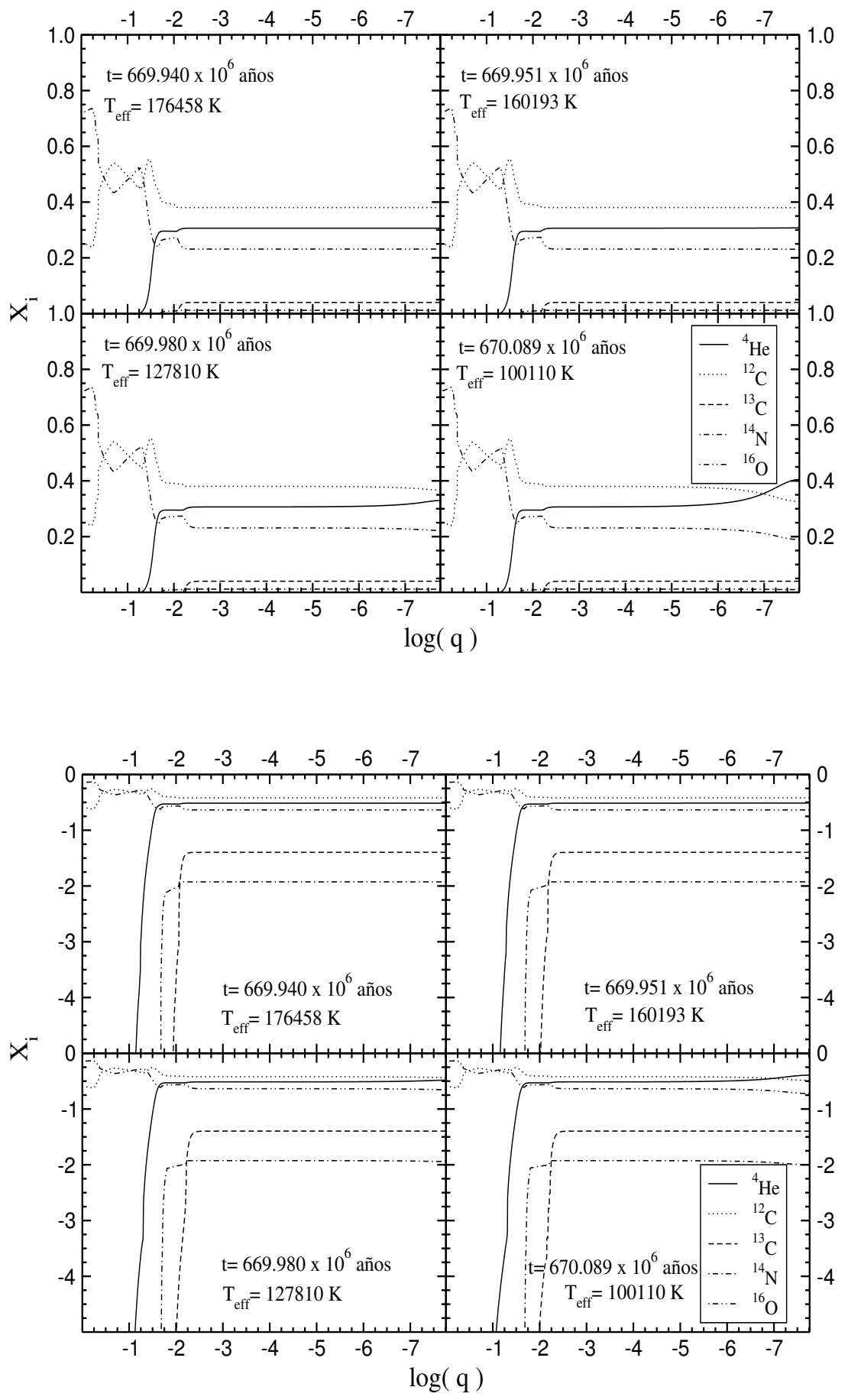

Figura 48: Figura superior: abundancias químicas internas de cuatro modelos distintos para una estrella con $\left(M_{Z A M S}, M_{W D}\right)=\left(2.7 M_{\odot}, 0.5885 M_{\odot}\right)$, en las primeras etapas de la rama de enfriamiento de enana blanca. Figura inferior: idem figura superior pero con las abundancias dadas en escala logarítmica. 

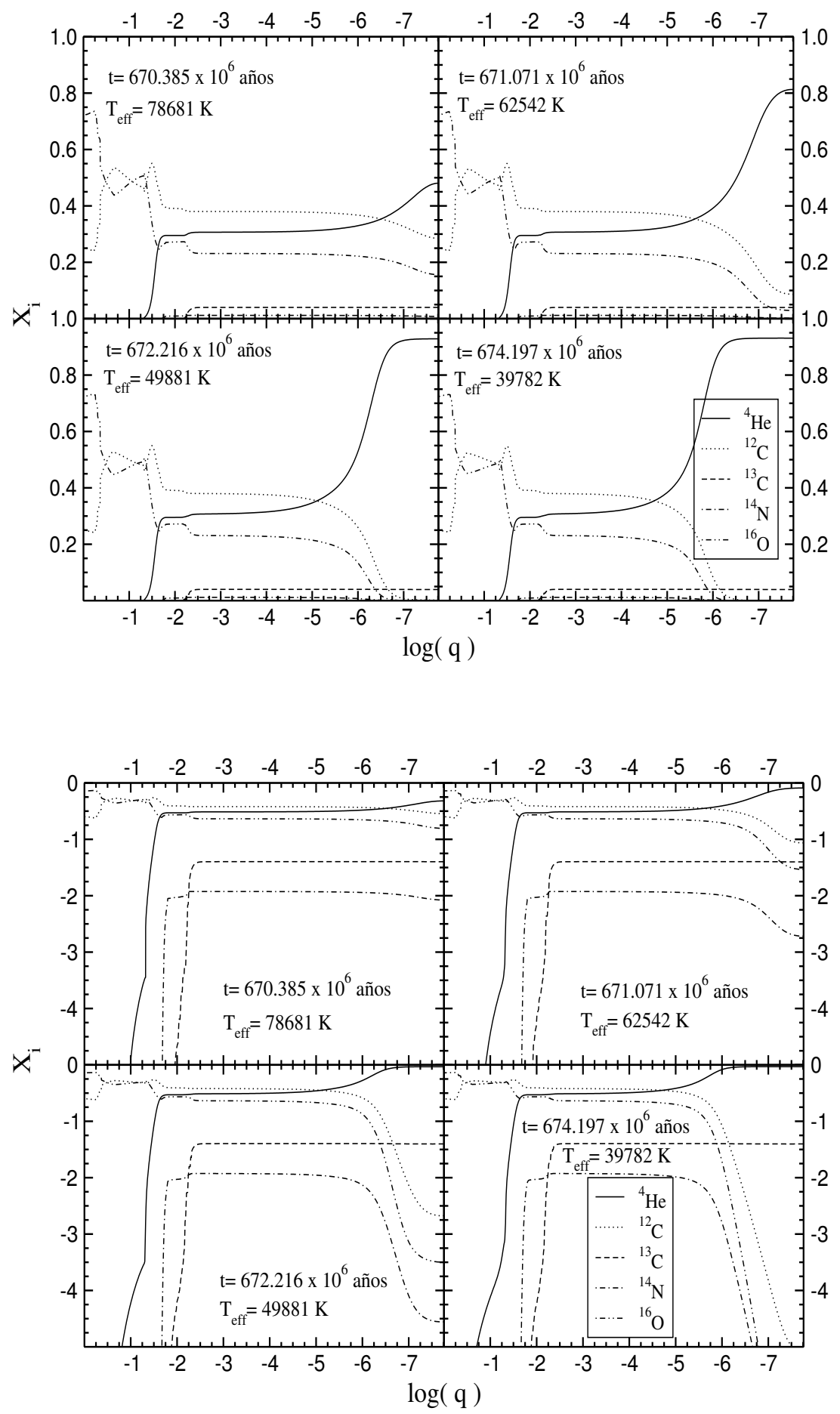

Figura 49: Figura superior: abundancias químicas internas de cuatro modelos distintos para una enana blanca DO con $\left(M_{Z A M S}, M_{W D}\right)=\left(2.7 M_{\odot}, 0.5885 M_{\odot}\right)$. Figura inferior: idem figura superior pero con las abundancias dadas en escala logarítmica. 


\subsection{Enanas blancas DB}

Estas estrellas se encuentran entre los 12000 y 30000K. Como señalaron Fontaine y Brassard (Fontaine and Brassard, 2002), la presencia de una estructura de doble capa tiene importantes implicaciones en los espectros pulsacionales de las DB pulsantes - ver (Althaus and Córsico, 2004).

El tratamiento de difusión empleado tiene en cuenta la difusión térmica. Esto no es una contribución despreciable al proceso de difusión, ya que la difusión térmica actúa en la misma dirección que el asentamiento gravitacional -fig. (50). Despreciar la difusión térmica es subestimar el ritmo para el cual el carbono y el oxígeno se difunden hacia dentro en la estrella - ver (Althaus and Córsico, 2004). La fig. (50) muestra un perfil interno de estrella DB de doble capa (Althaus and Córsico, 2004).

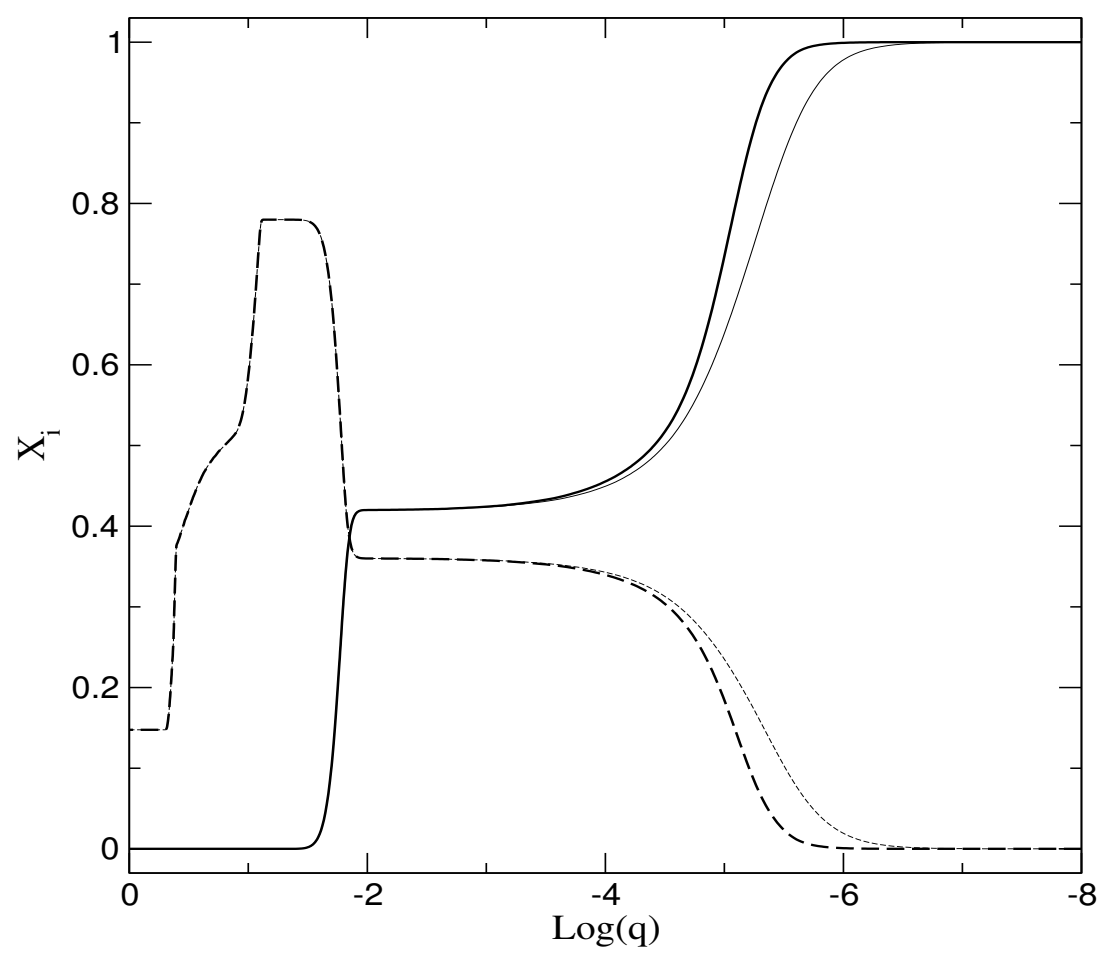

Figura 50: Abundancia fraccional por masa para ${ }^{4}$ He $y{ }^{12} \mathrm{C}$ (línea llena segmentada, respectivamente) como una función de la fracción de masa exterior q para un modelo de $D B$ de $0.60 M_{\odot}$ para una temperatura $T_{\text {eff }}=27300 \mathrm{~K}$. Las líneas gruesas (delgadas) corresponden al caso en el cual la difusión térmica es considerada (despreciada). Claramente se ve que la inclusión de la difusión térmica conduce a mantos puros de helio más gruesos (Althaus and Córsico, 2004).

En la fig. (51) se representan cuatro modelos con los perfiles internos de abundancias químicas para el dominio de las estrellas enanas blancas DB donde se aprecia la estructura de doble capa. 

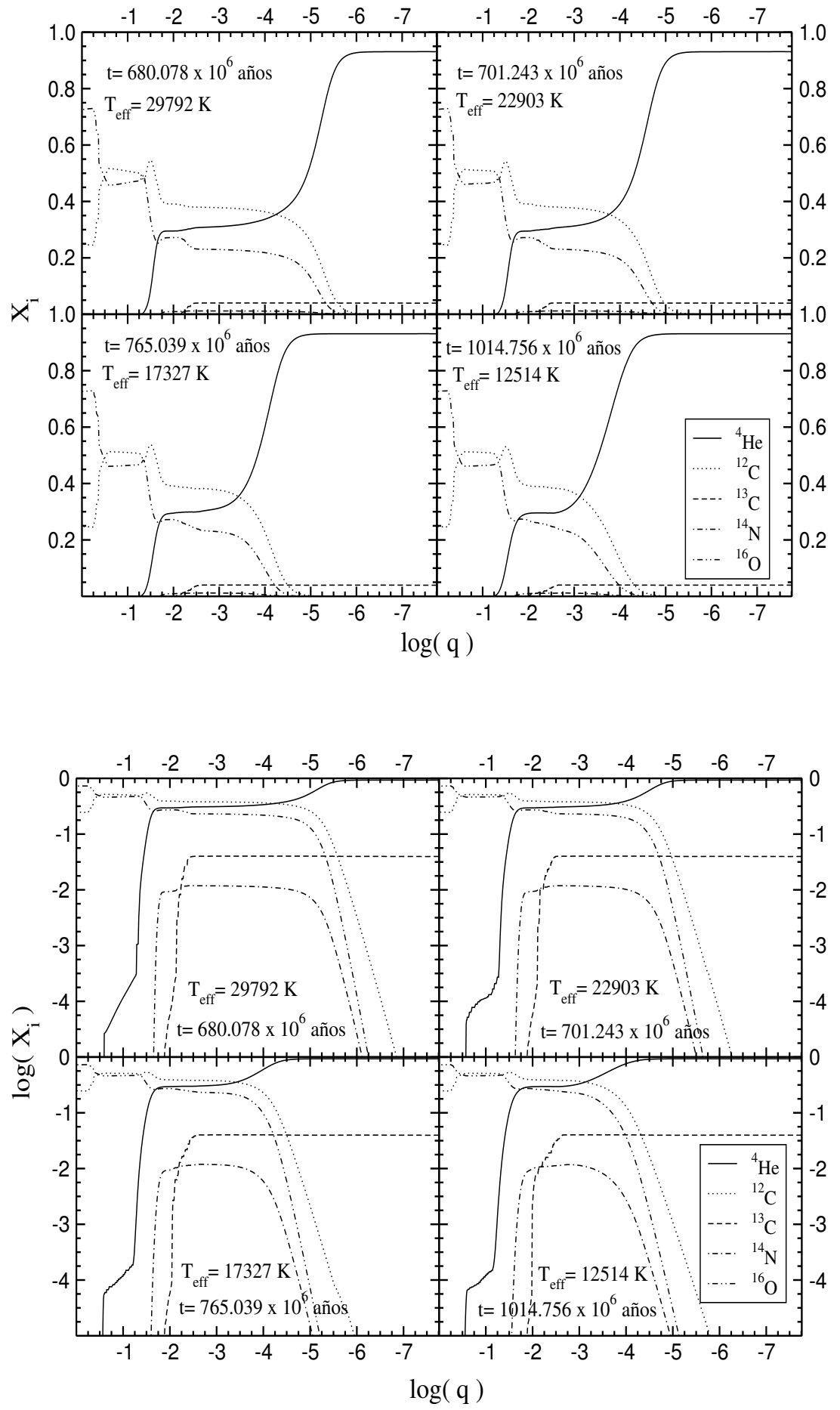

Figura 51: Figura superior: abundancias químicas internas de cuatro modelos distintos para una enana blanca $D B$ con $\left(M_{Z A M S}, M_{W D}\right)=\left(2.7 M_{\odot}, 0.5885 M_{\odot}\right)$. Figura inferior: idem figura superior pero con las abundancias dadas en escala logarítmica. 
En esta etapa el remanente posee bien desarrollada la estructura de doble capa con una envoltura rica en helio de $\approx 2.3 \times 10^{-6} M_{\odot}$, por encima de una envoltura remanente intermedia rica en helio, carbono y oxígeno, los restos de un pulso térmico de helio. Ambas envolturas yacen sobre un núcleo de carbono y oxígeno.

Otra característica importante es el episodio de mezcla que toma lugar en la región debajo de la zona de la intercapa en torno a un valor de $\log q=-1$. En efecto la región se carateriza por un peso molecular medio inducido que decrece hacia adentro debido a la aparición del overshooting durante la fase térmicamente pulsante AGB. La mezcla salt finger es la responsable de la redistribución de la abundancia química de la región.

Está claro que los procesos de difusión modifican en forma apreciable los perfiles químicos conforme evolucionan las enanas blancas, logrando que el grueso de helio flote hacia la superficie y los elementos pesados desciendan permaneciendo lejos de las capas superficiales. Por lo tanto se espera una formación de estructura de doble capa, en el momento en que las enanas blancas han alcanzado el dominio de la inestabilidad pulsacional [(Dehner and Kawaler, 1995); (Gautschy and Althaus, 2002); (Fontaine and Brassard, 2002)]. En este sentido, si la difusión actúa eficientemente, resulta que las capas externas son de helio puro, quedando por debajo de ésta una capa de helio, carbono y oxígeno.

Un hecho importante es que el perfil químico se va modificando apreciablemente con la evolución a través de la banda de inestabilbidad de las DB. Durante tales etapas los elementos de difusión no sólo espesan el manto de helio puro, sino que también modifican la forma del perfil químico. Notar que aún a etapas avanzadas el perfil químico está caraterizado por la estructura de doble capa.

\subsection{Enanas blancas DQ}

Luego de atravesar la etapa de las DB, el remanente estelar alcanza el dominio de las enanas blancas contaminadas de carbono de tipo espectral DQ. Para temperaturas menores de $12000 \mathrm{~K}$ hemos computado las etapas evolutivas de estos objetos. La composición química de dos modelos de enanas blancas DQ puede verse en la fig. (52).

Aún para estas etapas avanzadas la estrella posee una estructura de doble capa. La envoltura de helio puro tiene un valor de $1.9 \times 10^{-5} M_{\odot}$. Notar que se produce un enriquecimiento de las capas superficiales como un resultado del dredge up convectivo de la cola de carbono difundido, un proceso que se cree es el responsable del carbono observado de las DQ. 


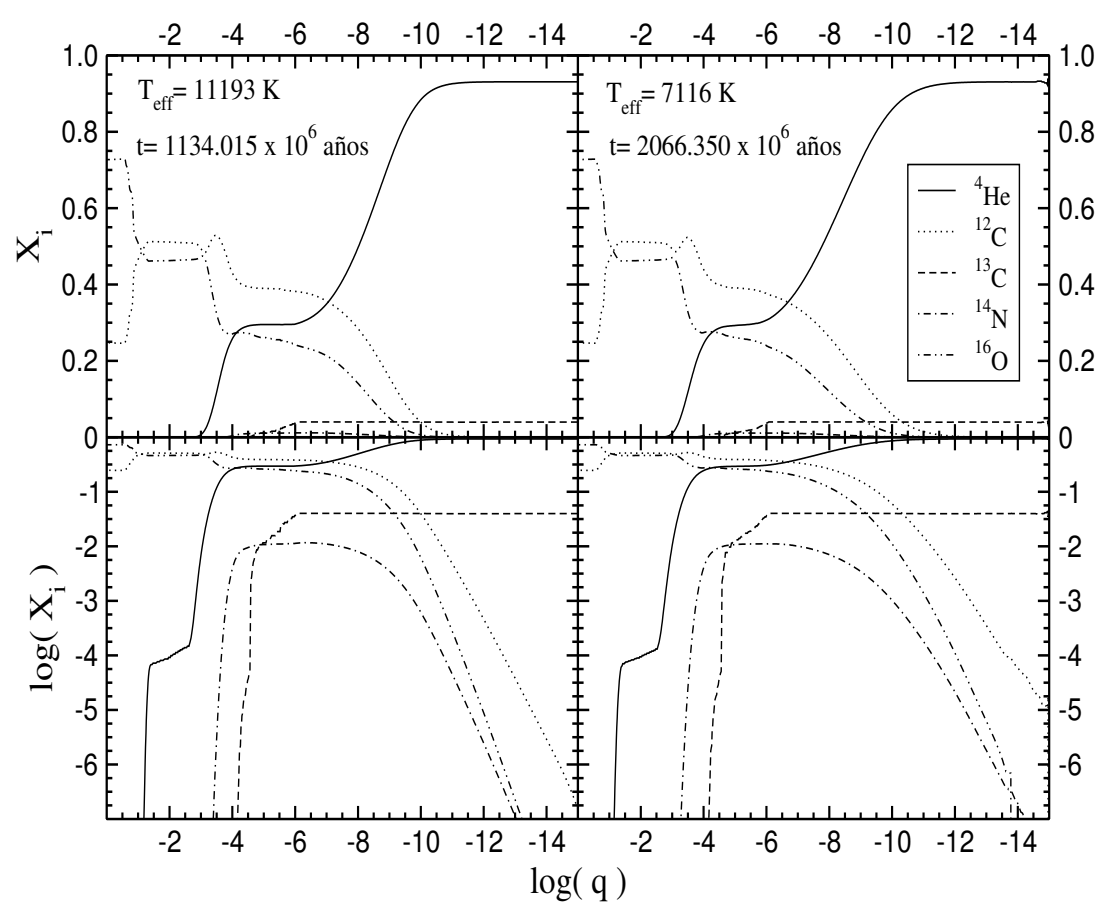

Figura 52: Figura superior: abundancias químicas internas de dos modelos distintos para una enana blanca $D Q$ con $\left(M_{Z A M S}, M_{W D}\right)=\left(2.7 M_{\odot}, 0.5885 M_{\odot}\right)$. Figura inferior: idem figura superior pero con las abundancias dadas en escala logarítmica.

\subsection{Una enana blanca DB: GD358}

Gracias a la astrosismología se puede determinar la relación carbono-oxígeno en el interior de las enanas blancas, y en particular en las enanas blancas DB. El caso más estudiado de este tipo de estrellas es la GD358. Se encuentra que la difusión conduce a la formación de una configuración de capa doble que lleva a una fuerte señal en el espectro de los modelos de la GD358. Fontaine y Brassard (Fontaine and Brassard, 2002) encontraron que sus modelos de capa doble con composiciones nucleares uniformes, son igualmente útiles para explicar los datos de una GD358 con una capa simple pero con composición nuclear variable. Las estrellas pulsantes DB con sus atmósferas de helio, se encuentran en un rango angosto de temperatura efectiva alrededor de $T_{\text {eff }} \sim 25000 \mathrm{~K}$. Nueve de estas estrellas son conocidas, y sus períodos están en un rango de 150 - 1100 segundos.

A partir de análisis astrosismológicos, realizados por Bradley y Winget (Bradley and Winget, 1994b) se estimó que la masa de la capa de helio en GD358 es de $\log \left(M_{\mathrm{He}} / M_{*}\right) \simeq-5.70$. Como es sabido las estrellas DQ son las descendientes más frías de las DB. En este sentido, en estrellas enanas blancas con atmósferas de helio de $T_{\text {eff }} \sim 10000 \mathrm{~K}$, se encontró con ante- 
rioridad, que la presencia de rastros de carbono en sus fotósferas podrían ser explicadas en términos de un efecto de dredge up en estrellas con $\log \left(M_{\mathrm{He}} / M_{*}\right) \simeq-3.5$ a -4.0 . Este valor es de unos 2 órdenes de magnitud mayor que la determinación astrosismológica en GD358 a $T_{\text {eff }} \sim 25000 \mathrm{~K}$, objeto que se presume es casi similar al antecesor de las estrellas DQ.

Encontramos modelos evolutivos muy detallados de estrellas enanas blancas DB, que incluyen difusión dependiente del tiempo realizados por Gautschy y Althaus (Gautschy and Althaus, 2002), existen dos zonas distinguibles de transición de composición química. Esto es, alrededor de un núcleo de carbono-oxígeno, existe un manto hecho de una mezcla de helio, carbono y oxígeno en proporciones aproximadamente iguales, los restos de la composición de la envoltura producidos por una evolución de born again post-AGB. Alrededor del manto, existe una envoltura de helio puro, formada por el asentamiento de carbono y oxígeno, un proceso que ocurre todavía en la fase evolutiva de las DB.

Para los modelos con difusión el perfil de composición química carateriza tres estructuras distintas que contribuyen a hacer un período espectral no-uniforme. Estos son: (1) la zona de transición de composición entre la envoltura de helio puro y el manto de helio-carbonooxígeno, (2) la zona de transición de composición entre el manto de helio-carbono-oxígeno y el núcleo de carbono-oxígeno y (3) la relación variable de carbono/oxígeno en el núcleo. Se supone que existe una conección evolutiva entre tipos espectrales de enanas blancas, la tan conocida relación PG1159-DB-DQ, la cual está basada sobre cálculos detallados de difusión dependientes con el tiempo acoplados a los cómputos evolutivos.

La inclusión de la difusión en la evolución de las enanas blancas DB indica que estas estrellas están todavía experimentando asentamiento de carbono y oxígeno y su estructura está caracterizada por una configuración química de doble capa. Esta propuesta de la doble capa explica naturalmente la conección evolutiva PG1159-DB-DQ. En comparación con los modelos de las DB "clásicas" caracterizados por una zona de transición de la composición de una capa sola, estas no se pueden conectar con la fase evolutiva previa de las PG1159, y están en conflicto con los modelos de su contraparte más fría, las estrellas DQ. La diferencia de la estructura mecánica entre los dos tipos de modelos (las de capa doble vs. las de capa simple) afectan a los períodos teóricos del espectro. 


\section{Conclusiones}

Argumentos teóricos y observacionales indican que el hidrógeno es el elemento más abundante en el universo, y que es el principal constituyente de la mayoría de las estrellas. Sin embargo, desde hace algún tiempo se han detectado estrellas que presentan ausencia o deficiencia de hidrógeno en sus atmósferas. Entre los objetos que presentan estas características, podemos mencionar las estrellas R Coronae Borealis, las estrellas centrales de nebulosas planetarias Wolf-Rayet y las PG1159, estas últimas son las estrellas más calientes que se conocen. En este contexto, el objetivo de la presente tesis ha estado enfocado hacia el estudio de aspectos evolutivos de las estrellas deficientes en hidrógeno. Nuestra motivación radica en el hecho que existen muy escasas simulaciones numéricas capaces de explicar convincentemente su formación y evolución. Además, estos objetos están captando un interés observacional entre los investigadores en parte porque representan un nexo evolutivo entre las estrellas supergigantes rojas y las enanas blancas.

Los resultados obtenidos en esta tesis están basados en la utilización del código de evolución estelar $\mathrm{LPCODE}^{6}$, desarrollado íntegramente en el Observatorio Astronómico de La Plata, el cual ha sido usado en numerosos trabajos por nuestro grupo de investigación. El código LPCODE ha sido perfeccionado y actualizado en su contenido de física a lo largo de varios años - ver las tesis doctorales (Althaus, 1996) y (Serenelli, 2002)-, y que sumado a las modificaciones realizadas para llevar a cabo la presente tesis, permite simular la evolución completa de una estrella de masa intermedia desde su etapa de secuencia principal hasta su evolución final de enana blanca.

Los resultados de mayor relevancia aportados por esta tesis son:

1. Se calculó la evolución completa desde la secuencia principal pasando por todas las etapas intermedias hasta arribar a la configuración de enana blanca con deficiencia en hidrógeno, computando su evolución en forma autoconsistente con los cambios químicos y sobre la base de un tratamiento completo y detallado de los procesos físicos que ocurren en un interior estelar. Cabe remarcar que los estudios existentes en la literatura no cubren todas las etapas evolutivas estudiadas aquí de manera integral. Por lo tanto, es la primera vez que modelos evolutivos de enanas blancas DB son calculados teniendo en cuenta la historia evolutiva completa de la estrella progenitora.

2. Se ha explorado por primera vez la teoría extendida de convección denominada GNA para fluidos con gradiente de composición química en estrellas de masa intermedia. ${ }^{7}$

\footnotetext{
${ }^{6} \mathrm{El}$ código LPCODE está escrito en lenguaje de programación FORTRAN 77, el cual posee más de 27000 líneas.

${ }^{7}$ La teoría de la GNA fue aplicada sólo para estrellas masivas por los autores que la desarrollaron -ver
} 
Hemos encontrado que esta teoría reproduce aspectos observacionales tales como el fenómeno Hot Bottom Burning y el $3^{\text {er }}$ dredge up los cuales tienen lugar durante la fase AGB. En particular nuestros cálculos predicen la formación de estrellas de baja masa ricas en carbono en buen acuerdo con las observaciones. Por otra parte, encontramos que la GNA predice naturalmente edades para objetos born again mucho más cortas (y por ende en mejor acuerdo con la escala de tiempo observada para los objetos Sakurai) que las predichas por la teoría standard de la mixing length.

3. Como consecuencia de los procesos de difusión que operan durante el régimen de enana blanca, encontramos que el perfil químico de la envoltura que caracterizaría a las enanas blancas pulsantes DB, tendría una configuración de doble capa. Esto tendría implicaciones importantes para las propiedades pulsacionales de estas estrellas, como ha sido sugerido recientemente por Fontaine y Brassard (Fontaine and Brassard, 2002). En efecto, estos autores han demostrado que la presencia de una configuración de doble capa en estas estrellas podría modificar sustancialmente las actuales inferencias astrosismológicas acerca de la sección eficaz de la reacción ${ }^{12} \mathrm{C}(\alpha, \gamma){ }^{16} \mathrm{O}$.

4. Nuestros resultados indican que el proceso de born again es, en efecto, un mecanismo eficiente para explicar la existencia de enanas blancas deficientes en hidrógeno. Específicamente encontramos que la masa de la envoltura de hidrógeno es reducida de $\approx 10^{-4}$ a $1.3 \times 10^{-8} M_{\odot}$ en sólo un mes.

Con relación a lo hecho por otros autores vemos que, nuestros cálculos refuerzan resultados obtenidos por el grupo de investigación del Instituto Max Planck. Sin embargo, la investigación llevada a cabo en esta tesis es mucho más general, que la realizada por el mencionado grupo, y cubre, como se mencionó, todas las fases evolutivas que llevan a la formación de una estrella deficiente en hidrógeno, aún hasta su estado final de enana blanca.

Específicamente hemos calculado, en forma completa, la evolución de un modelo estelar con masa inicial de $2.7 M_{\odot}$ desde la secuencia principal a través de la fase AGB y pérdida de masa, pasando por el escenario de born again hacia la etapa de enana blanca, terminando como un remanente de $0.5885 M_{\odot}$. Los resultados obtenidos cubren varias etapas evolutivas de difícil tratamiento numérico como son los pulsos térmicos o la fase de born again. Este escenario, se produce a partir de un pulso térmico de helio muy tardío ocurrido en la rama de enfriamiento temprana de las enanas blancas, después de que la quema de hidrógeno ha cesado. La inclusión de un esquema dependiente con el tiempo para el tratamiento simultáneo de la evolución nuclear y los procesos de mezcla debido a la convección, salt finger y overshooting difusivo, ha permitido un estudio detallado de los cambios de abundancias a por ejemplo (Grossman and Taam, 1996). 
través de todas las fases evolutivas, particularmente durante la fase térmicamente pulsante de la AGB y el episodio extremadamente corto del born again, para las cuales un esquema instantáneo de mezcla, es totalmente inadecuado.

A partir de los resultados obtenidos podemos mencionar las siguientes características:

- En la etapa correspondiente a la existencia del núcleo de helio y la fase térmicamente pulsante observamos:

1. La ocurrencia del OV durante la quema central de helio conduce a una discontinuidad en el perfil de oxígeno. Esto está en muy buen acuerdo con cálculos realizados por Straniero y colaboradores (Straniero et al., 2003), quienes han reportado que la existencia de episodios extra de mezcla conduce a discontinuidades en los perfiles químicos del núcleo de las estrellas de masa intermedia. Este es un punto importante teniendo en cuenta que es precisamente durante la quema central de helio cuando se forma el núcleo de carbono-oxígeno que finalmente caracterizará a la estrella enana blanca. La importancia de tales episodios de mezcla, particularmente el OV, ha sido recientemente enfatizada por nuestro grupo de investigación -ver (Althaus et al., 2003)- quienes encuentran que la ocurrencia de OV durante el estado de quema central de helio afecta marcadamente al espectro teórico pulsacional de las enanas blancas masivas ZZ Ceti. Los modelos estelares aquí desarrollados serán de aplicación inmediata, por nuestro grupo, al estudio de las propiedades pulsacionales de enanas blancas de masa intermedia. La comparación de las predicciones teóricas con la observación de enanas blancas pulsantes, nos permitirá obtener conclusiones sobre la ocurrencia o no de episodios de OV en los progenitores de enanas blancas.

2. Encontramos que la región de intercapa posee abundante oxígeno. Esto es consecuencia de la mezcla de OV que tiene lugar durante el pico del pulso térmico, detalle que es de importancia y para tener en cuenta en etapas evolutivas posteriores cuando se exponen en la superficie estelar las abundancias de dicha intercapa.

3. Encontramos la formación de pequeños reservorios de ${ }^{13} \mathrm{C}$ y ${ }^{14} \mathrm{~N}$ en la base de la capa de helio puro, después del final de la fase de dredge up. Durante el período del interpulso, la zona de ${ }^{13} \mathrm{C}$ es quemada radiativamente, mientras que la zona de ${ }^{14} \mathrm{~N}$ es "tragada" por la zona convectiva del flash de helio durante el próximo pulso térmico. El flujo de neutrones que resulta de la quema de estos elementos mediante la captura de partículas $\alpha$, constituye el flujo requerido de neutrones para la formación de elementos más pesados que el hierro a través del llamado proceso lento de captura. Nuestros resultados muestran que, para obtener una 
abundancia de ${ }^{13} \mathrm{C}$ y ${ }^{14} \mathrm{~N}$ lo suficientemente alta como para producir un flujo de neutrones apreciable, se requiere la existencia de procesos de mezcla adicionales más allá de lo que predice el criterio de Schwarzschild.

- Luego de la etapa de los pulsos térmicos sucede lo que se conoce como born again. Esta etapa no ha sido estudiada por otros autores, salvo en los trabajos de Herwig quien últimamente ha realizado cálculos detallados sobre la misma. En esta fase podemos mencionar, además de los ya mencionados, los siguientes hechos:

1. La ocurrencia de un pulso térmico tardío responsable de la quema de protones remanentes en la estrella. Reforzando así, los resultados obtenidos por Herwig.

2. Después del episodio born again, nuestros modelos predicen un doble loop en el diagrama $\mathrm{H}-\mathrm{R}$; esto es la estrella alcanza las dimensiones de gigante roja por segunda vez después de que ha ocurrido el último pulso térmico y antes de que finalmente arribe a la rama de enfriamiento de las enanas blancas. La primera vez la estrella evoluciona rápidamente hacia la AGB, vuelve al azul, y luego evoluciona más lentamente hacia la AGB por segunda vez, antes de tomar finalmente la rama enfriamiento de enana blanca. La existencia de un doble loop abre la interesante posibilidad de interpretar al objeto Sakurai y a la estrella FG Sagitae ${ }^{8}$ como pertenecientes a un mismo canal evolutivo pero en distintas etapas del mismo. En efecto, el primero ha estado evolucionando hacia la AGB por primera vez después de la ocurrencia del ultimo pulso térmico, mientras que el objeto FG Sge ha sido observado durante su segundo retorno a la AGB.

3. Después del episodio born again, ${ }^{4} \mathrm{He},{ }^{12} \mathrm{C}$ y ${ }^{16} \mathrm{O}$ son por lejos las especies más dominantes en la envoltura con una abundancia por masa de $\left({ }^{4} \mathrm{He},{ }^{12} \mathrm{C},{ }^{16} \mathrm{O}\right)=$ $(0.306,0.376,0.228)$. Mientras que para los siguientes núcleos tenemos las cantidades $\left({ }^{13} \mathrm{C},{ }^{14} \mathrm{~N},{ }^{22} \mathrm{Ne}\right)=(0.040,0.012,0.021)$. Esto está en acuerdo con abundancias superficiales observadas en la mayoría de las estrellas post-AGB deficientes en hidrógeno como las PG1159. En particular encontramos que la alta abundancia de oxígeno encontrada, es reproducible por los modelos estelares, si se invocan episodios de mezcla extras en la envoltura, como por ejemplo overshooting. Este resultado es una indicación de que el OV, cuya existencia en los interiores estelares ha sido debatida durante largos años, juega un rol preponderante en la evolución estelar. Otra predicción de nuestros modelos estelares, es la elevada abundancia

\footnotetext{
${ }^{8}$ Recordemos que esta estrella comenzó a ser observada en el año 1880 y que a lo largo de los cien años siguientes ha incrementado su radio en tres órdenes de magnitud.
} 
de ${ }^{14} \mathrm{~N}$, lo que está en acuerdo con lo observado por Dreizler y Heber (Dreizler and Heber, 1998) en algunas PG1159.

4. Otra característica encontrada está relacionada a la eficiencia de OV. En particular una gran eficiencia de OV $(f=0.03)$ predice que la fracción de masa superficial de ${ }^{14} \mathrm{~N}$ es reducida a $10^{-6}$ y las especies superficiales principales cambian a $\left({ }^{4} \mathrm{He},{ }^{12} \mathrm{C},{ }^{16} \mathrm{O}\right)=(0.27,0.42,0.277)$. En particular, la abundancia de oxígeno obtenida en este caso es bastante más grande que la observada. Concluimos entonces que solamente un OV moderado predice un buen acuerdo con las observaciones.

5. Durante la etapa temprana de PG1159 alrededor de $3 \times 10^{-4} M_{\odot}$ de ${ }^{13} \mathrm{C}$ es procesado mediante la reacción ${ }^{13} \mathrm{C}(\alpha, n){ }^{16} \mathrm{O}$. El helio es reducido de $8.5 \times 10^{-3}$ a $5.2 \times 10^{-4} M_{\odot}$ como resultado de la quema de helio.

- Con respecto a los efectos del esquema de difusión empleado para los elementos químicos, podemos citar:

1. La difusión de elementos químicos conduce a una estructura de doble capa durante la evolución de las estrellas DB. En estos remanentes estelares tenemos una capa de helio puro que yace sobre una de helio, carbono y oxígeno, la cual descanza sobre el núcleo inerte de carbono-oxígeno.

Finalmente, quisiera mencionar que los modelos obtenidos en este trabajo de tesis doctoral, permitirán a nuestro grupo de investigación encarar, entre otras, las siguientes cuestiones y aplicaciones:

1. Es bien sabido que las enanas blancas muy luminosas emiten una gran cantidad de neutrinos provenientes de las regiones centrales muy calientes. Por ejemplo, la emisión de neutrinos constituye el principal mecanismo de enfriamiento de la enana blanca durante sus primeras etapas evolutivas. Esto abre la posibilidad de utilizar a estas estrellas para estudiar propiedades de los neutrinos. Esto es posible gracias a la existencia de enanas blancas pulsantes luminosas para las cuales muchos de sus períodos de oscilación han sido medidos con gran precisión.

2. El descubrimiento de que muchas PG1159 pulsan, abre un camino importante al estudio de estas estrellas y también de su historia evolutiva previa. Los modelos evolutivos desarrollados en esta tesis, dado su gran detalle, serán de utilidad para permitir estudiar las propiedades pulsacionales de estas estrellas y tratar de entender unos de los problemas no resueltos tales como la variación temporal del período $(\dot{P})$. La estrella PG1159-035 tiene medido el $\dot{P}$. Pero la medición relizada da un $\dot{P}$ de un orden de 
magnitud más grande que el esperado teóricamente. Una posibilidad para explicar ese valor observado es suponer que dicha estrella experimenta una tasa de pérdida de masa que la hace evolucionar más rápidamente exhibiendo así un $\dot{P}$ más grande. Es nuestra intención explicar esta posibilidad en base a los modelos de las estrellas PG1159 presentados en esta tesis.

3. Se podrá estudiar en detalle la nucleosíntesis que lleva a la formación de elementos pesados durante el born again. Esto sería importante porque podríamos compararlo con la formación de elementos pesados observada en los objetos Sakurai.

4. Otro estudio futuro concierne a los aspectos evolutivos de las enanas blancas DB, los cuales requieren un conocimiento detallado de la evolución de sus progenitores. En particular, la formación de una estructura de doble capa y en especial las consecuencias para las propiedades pulsacionales de las enanas blancas variables DBV está dentro de los objetivos de nuestro grupo de investigacion. 


\section{Apéndice A: Conceptos astrofísicos}

\subsection{Ecuación de equilibrio hidrostático}

Consideremos una capa esférica y delgada en equilibrio. La misma experimenta una fuerza debido a la gravedad (hacia el centro de la estrella) y otra debido a la diferencia de presión entre ambas superficies de la capa esférica (hacia afuera, pues la presión en la cara inferior es mayor que en la superior); ambas por unidad de volumen. Entonces:

$$
\frac{\partial P}{\partial r}+g \varrho=0
$$

la cual da la condición de equilibrio hidrostático como:

$$
\frac{\partial P}{\partial r}=-g \varrho
$$

Notar que $P$ decrece con el aumento de $r$. Esto muestra el balance entre las fuerzas de presión y gravedad.

Dentro de un cuerpo simétricamente esférico la aceleración de gravedad $g$ a una distancia $r$ del centro está dada por:

$$
g=\frac{G m}{r^{2}}
$$

donde $G$ es la constante newtoniana de la gravitación, $m$ la masa dentro del radio $r$. Usando esto en la ec. (26) obtenemos:

$$
\frac{\partial P}{\partial r}=-\frac{G m}{r^{2}} \varrho
$$

Si tomamos $m$ como variable independiente en lugar de $r$, obtenemos la condición de equilibrio hidrostático. De la ecuación de conservación de la masa:

$$
\frac{\partial m}{\partial r}=4 \pi r^{2} \varrho
$$

entonces si multiplicamos por su inversa:

$$
\frac{\partial r}{\partial m}=\frac{1}{4 \pi r^{2} \varrho}
$$

obteniendo, la ecuación de equilibrio hidrostático en forma Lagrangeana:

$$
\frac{\partial P}{\partial m}=-\frac{G m}{4 \pi r^{4}} .
$$




\subsection{Transporte de energía por radiación, conducción y convec- ción}

\subsubsection{Transporte de energía radiativa}

El camino libre medio de un fotón $l_{f o t}$ dentro de una estrella como el sol, está dado por:

$$
l_{\text {fot }}=\frac{1}{\kappa \varrho}
$$

donde $\varrho$ la densidad del medio y $\kappa$ es el coeficiente de absorción media, esto es la sección eficaz por unidad de masa promediada sobre la frecuencia. El valor típico para el Sol es de $l_{\text {fot }}=2 \mathrm{~cm}$ (es decir la materia estelar es muy opaca), con $\kappa=0.4 \mathrm{~cm}^{2} \mathrm{~g}^{-1}$, así $l_{\text {fot }} / R_{\odot} \approx$ $3 \times 10^{-11}$. En este caso, el transporte puede ser tratado como un proceso de difusión. El flujo difusivo $\boldsymbol{j}$ (por unidad de área y tiempo) entre lugares de diferente densidad de partícula $n$ está dado por:

$$
j=-D \cdot \nabla n
$$

donde D es el coeficiente de difusión,

$$
D=\frac{1}{3} v l_{p}
$$

determinado por los valores medios de la velocidad media $v$ y el camino libre medio de las partículas $l_{p}$.

Para obtener el flujo difusivo correspondiente a la energía radiativa $\boldsymbol{F}$, reemplazamos $n$ por la densidad de energía de radiación $U$ en ec. (33) y $\boldsymbol{j}$ por $\boldsymbol{F}$,

$$
\begin{gathered}
\boldsymbol{F}=-D \cdot \nabla U, \\
\operatorname{con} \quad U=a T^{4},
\end{gathered}
$$

$v$ por la velocidad de la luz $c$, y $l_{p}$ por $l_{f o t}$. En ec. (36), a es la constante de densidad de radiación. Por la simetría esférica del problema, $\boldsymbol{F}$ tiene sólo componente radial $F_{r}=|\boldsymbol{F}|$ $=F$ y por lo tanto $\nabla U$ se reduce a la derivada en la dirección radial

$$
\frac{\partial U}{\partial r}=4 a T^{3} \frac{\partial T}{\partial r}
$$


quedando entonces:

$$
F=-\frac{4 a c}{3} \frac{T^{3}}{\kappa \varrho} \frac{\partial T}{\partial r}
$$

Nótese que esto puede ser interpretado como una ecuación de conducción de calor mediante

$$
\boldsymbol{F}=-k_{r a d} \cdot \nabla T
$$

donde

$$
k_{\text {rad }}=\frac{4 a c}{3} \frac{T^{3}}{\kappa \varrho}
$$

representa el coeficiente de conducción para este transporte radiativo, o mejor usando $\kappa_{\text {rad }}$ en lugar de $\kappa$ para distinguirlo del coeficiente que definiremos más adelante en el caso de transporte conductivo.

$$
k_{\text {rad }}=\frac{4 a c}{3} \frac{T^{3}}{\kappa_{\text {rad }} \varrho}
$$

De ec. (38) para el gradiente de temperatura y reemplazando $F$ por la luminosidad local $l=4 \pi r^{2} F$; tenemos

$$
\frac{\partial T}{\partial r}=-\frac{3}{16 \pi a c} \frac{\kappa_{r a d} \varrho l}{r^{2} T^{3}}
$$

Después de la transformación para la variable independiente $m$, ec. (30) se obtiene:

$$
\frac{\partial T}{\partial m}=-\frac{3}{64 \pi^{2} a c} \frac{\kappa_{r a d} l}{r^{4} T^{3}}
$$

Esta ecuación no es válida cuando uno se aproxima a la superficie de la estrella, debido a que la densidad decrece haciendo cambiar el camino libre medio del fotón, volviéndose éste comparable a la distancia hacia la superficie, y por lo tanto no vale la aproximación de difusión. 


\subsubsection{Transporte de energía conductiva}

En la conducción del calor, la transferencia de energía ocurre mediante colisiones durante el movimiento térmico aleatorio de las partículas. Para la materia no-degenerada la conducción no es importante en el transporte de energía. El camino libre medio es varios órdenes de magnitud menor que aquel para los fotones (con densidades de $\varrho \approx 1.4 \mathrm{~g} \mathrm{~m}^{-3}$ en el sol); y la velocidad de las partículas es sólo de unos centésimos de $c$. Por lo tanto el coeficiente de difusión ec. (34) es mucho menor que aquel para los fotones.

La situación se vuelve completamente diferente para los núcleos de las estrellas evolucionadas, donde el gas de electrones está altamente degenerado. La degeneración aumenta el camino libre medio considerablemente. Entonces el coeficiente de difusión es grande.

El flujo de energía $\boldsymbol{F}_{\text {cond }}$ debido a la conducción de calor puede ser escrito como:

$$
\boldsymbol{F}_{\text {cond }}=-k_{\text {cond }} \cdot \nabla T .
$$

Análogamente a la ec. (41) escribimos el coeficiente conductivo $k_{\text {cond }}$ como,

$$
k_{\text {cond }}=\frac{4 a c}{3} \frac{T^{3}}{\kappa_{\text {cond }} \varrho}
$$

donde hemos definido la "opacidad conductiva" $\kappa_{\text {cond }}$.

\subsubsection{Gradiente radiativo}

La suma del flujo conductivo $\boldsymbol{F}_{\text {cond }}$ ec. (44) y el radiativo $\boldsymbol{F}_{\boldsymbol{r a d}}$ ec. (39) está dado por

$$
\boldsymbol{F}=\boldsymbol{F}_{\text {rad }}+\boldsymbol{F}_{\text {cond }}=-\left(k_{\text {rad }}+k_{\text {cond }}\right) \cdot \boldsymbol{\nabla} T,
$$

Entonces de ec. (46) tenemos

$$
\boldsymbol{F}=-\frac{4 a c}{3} \frac{T^{3}}{\varrho}\left(\frac{1}{\kappa_{\text {rad }}}+\frac{1}{\kappa_{\text {cond }}}\right) \cdot \nabla T,
$$

y definimos $1 / \kappa$ a la inversa de la opacidad total como, la suma de las inversas de las opacidades radiativa y conductiva, esto es:

$$
\frac{1}{\kappa}=\frac{1}{\kappa_{\text {rad }}}+\frac{1}{\kappa_{\text {cond }}}
$$


reescribiendo la ec. (43) considerando la opacidad total,

$$
\frac{\partial T}{\partial m}=-\frac{3}{64 \pi^{2} a c} \frac{\kappa l}{r^{4} T^{3}}
$$

Para obtener el gradiente de temperatura radiativo hacemos el cociente de ec. (49) sobre ec. (31) obteniendo (notar que se emplea el $\kappa$ y no el $\kappa_{\text {rad }}$ ):

$$
\frac{(\partial T / \partial m)}{(\partial P / \partial m)}=\frac{3}{16 \pi a c G} \frac{\kappa l}{m T^{3}} .
$$

Llamamos al cociente de derivadas como $(d T / d P)_{\text {rad }}$, y con eso queremos representar la variación de la temperatura $\mathrm{T}$ con la profundidad, donde la profundidad está expresada por la presión, la cual crece monótonamente hacia adentro. En este sentido, una estrella que está en equilibrio hidrostático y el transporte de energía es por radiación (y conducción), $(d T / d P)_{\text {rad }}$ es un gradiente que describe la variación de temperatura con la profundidad. Si usamos la notación acostumbrada:

$$
\begin{gathered}
\nabla_{r a d}=\left(\frac{d \ln T}{d \ln P}\right)_{r a d}, \\
\nabla_{r a d}=\frac{3}{16 \pi a c G} \frac{\kappa l P}{m T^{4}} .
\end{gathered}
$$

en donde se han incluido los efectos de la conducción.

\subsubsection{Gradiente adiabático}

Definimos el gradiente de temperatura adiabático $\nabla_{a d}$, por la cantidad:

$$
\nabla_{a d}=\left(\frac{\partial \ln T}{\partial \ln P}\right)_{s},
$$

donde el subíndice $s$ indica que la definición es válida a entropía constante, puesto que para cambios adiabáticos la entropía tiene que permanecer constante. El $\nabla_{a d}$ podemos escribirlo también como:

$$
\nabla_{a d}=\left(\frac{P}{T} \frac{d T}{d P}\right)_{s}=\frac{P \delta}{T \varrho c_{P}}
$$




$$
\text { donde } \quad \delta=-\left(\frac{\partial \ln \varrho}{\partial \ln T}\right)_{P}=\frac{T}{v}\left(\frac{\partial v}{\partial T}\right)_{P},
$$

$\varrho$ es la densidad y sale de la ecuación de estado $\varrho=\varrho(P, T), v=1 / \varrho$ es el volumen específico (definido por unidad de masa); y

$$
c_{P}=\left(\frac{d q}{d T}\right)_{P}
$$

es el calor específico a presión constante -ver (Kippenhahn and Weigert, 1990).

Notar la diferencia en definición y en significado de $\nabla_{r a d}$ ec. (52) y de $\nabla_{a d}$ introducido en la ec. (54), la cual no sólo corresponde a sus valores numéricos. El $\nabla_{\text {rad }}$ significa una derivada espacial (conectando a $P$ y $T$ entre dos capas de masa vecinas), mientras que $\nabla_{a d}$ describe la variación térmica de un único elemento de masa durante su compresión adiabática. Solamente en casos especiales tienen el mismo valor, y en ese caso hablamos de una "estratificación adiabática".

\subsection{Inestabilidad dinámica}

El criterio de estabilidad dinámica o criterio de Schwarzschild (llamado así después de Karl Schwarzschild) es el siguiente:

$$
\nabla_{\text {rad }}<\nabla_{a d}
$$

en ese caso la capa radiativa es estable. Pero si en el criterio, ec. (57), el miembro izquierdo es mayor que el derecho, la capa es dinámicamente inestable. Si son iguales uno habla de estabilidad marginal. Esto ocurre en el interior de las estrellas evolucionadas, donde los elementos pesados son generalmente producidos debajo de los más livianos, de tal manera que el peso molecular $\mu$ aumenta hacia adentro (como lo hace la presión).

Si este criterio ocurre entonces favorece la estabilidad, luego los movimientos convectivos no van a ocurrir, y todo el flujo será llevado por radiación, esto es el gradiente actual $\nabla=\nabla_{\text {rad }}$. Pero si el criterio favorece a la inestabilidad, entonces pequeñas perturbaciones aumentarán una amplitud finita hasta que toda la región se mezcla con movimientos convectivos que llevan parte del flujo. Esta inestabilidad puede ser causada o por el hecho que $\nabla_{\text {rad }}$ se haya vuelto muy grande (gran flujo o materia muy opaca) o por una disminución del $\nabla_{a d}$, ambos casos ocurren en las estrellas. 


\subsection{Overshoot (OV)}

Consideremos el caso en las cercanías y fuera del límite de una zona convectiva (imaginemos que estamos por encima de esa zona convectiva), cuya masa (la de esa pequeña región) es $M_{\text {lim.conv. }}$, como la calculada en ausencia de OV. El límite está definido como una superficie de estabilidad neutra, donde:

$$
\nabla_{r a d}=\nabla_{a d}
$$

de acuerdo al criterio clásico, ec. (57); (sin perder generalidad lo pensaremos para una estrella de la ZAMS, es decir de composición homogénea). Se asume mezcla completa y una estratificación adiabática con $\nabla=\nabla_{a d}+\varepsilon(0<\varepsilon \ll 1)$ en la región convectiva debajo de $M_{\text {lim.conv. }}$ mientras que asumimos que no hay mezcla y que $\nabla=\nabla_{\text {rad }}$ para la región radiativa sobre $M_{\text {lim.conv. }}$

Este modelo implica un problema obvio: el límite entre los regímenes en los cuales los movimientos convectivos están presentes $(v>0)$ y ausentes $(v=0)$ es determinado por el criterio ec. (58), los cuales esencialmente dependen de las fuerzas de flotabilidad, y por lo tanto describe la aceleración $\dot{v}$ más que la velocidad $v$. Los elementos ascendentes de convección son acelerados hasta que ellos han alcanzado $M_{\text {lim.conv. }}$; el frenado comienza más allá de este borde, el cual es pasado por los elementos debido a su inercia.

\subsection{Semiconvección}

Las inestabilidades doble difusivas aparecen cuando el transporte de dos propiedades diferentes compiten una en contra de la otra para dominar la estabilidad del fluido. En las estrellas, el calor y la composición son las dos cantidades transportadas por la mezcla. la semiconvección ocurre cuando un fluido conducido térmicamente tiene un gradiente que se opone a la inestabilidad. En las estrellas, la quema nuclear provee la fuente de calor para la conducción térmica. Esto también causa cambios de composición química, como los casos en que los elementos son transformados en especies de mayor peso molecular, conduciendo a la posibilidad de estabilizar el gradiente de composición.

Como hemos dicho, un fluido de composición homogénea es inestable para la convección si su gradiente de temperatura es mayor que el gradiente adiabático:

$$
\nabla-\nabla_{a d}>0
$$

donde: 


$$
\nabla=\frac{\partial \ln T}{\partial \ln P}
$$

es el gradiente logarítmico de temperatura, y $\nabla_{a d}$ es la expresión dada en ec. (53). En las estrellas, en ciertas etapas evolutivas, la convección es tan rápida comparada con los tiempos de escala que hace que el proceso sea prácticamente instantáneo.

¿Qué pasaría si una estrellas es inestable según el criterio Schwarzschild en una región con un marcado gradiente de composición? Si el gradiente de composición que se opone a la inestabilidad térmica satisface la relación:

$$
\left(\nabla-\nabla_{a d}\right)-\nabla_{\mu}<0
$$

el rápido crecimiento de los movimientos convectivos se estabiliza, donde $\nabla_{\mu}$ es:

$$
\nabla_{\mu}=\frac{\partial \ln \mu}{\partial \ln P}
$$

es el gradiente de composición sin dimensiones, donde la composición está medida por el peso molecular $\mu$. Este es el criterio de estabilidad de Ledoux (Ledoux, 1947).

Consideremos una burbuja de fluido que se desplaza hacia arriba desde su posición de equilibrio en un medio con gradientes de temperatura y composiciones tales que el fluido es Ledoux estable y Schwarzschild inestable. La burbuja desplazada será más caliente que su entorno puesto que la inestabilidad Schwarzschild y la flotabilidad térmica la llevarán hacia arriba. Ella será también más pesada que su entorno, y por lo tanto sentirá una fuerza hacia abajo. Puesto que el fluido es Ledoux estable, la fuerza hacia abajo gana apareciendo una fuerza restauradora neta, esto es la burbuja oscila entorno a su posición de equilibrio. Si hay una tasa no nula de difusión térmica entonces mientras la burbuja está sobre su excursión hacia arriba donde es más caliente que su entorno, ésta pierde calor y vuelve a su posición de equilibrio con una temperatura menor que con la que comenzó. Cuando hace su excusión hacia abajo, la flotabilidad negativa la hará viajar algo más profundo que la que podría tener en cualquier otro caso. Así, la amplitud de la oscilación crece. Esta inestabilidad vibracional o sobreestabilidad crece en un tiempo de escala de difusión térmica. Esto es lo que aquellos que estudian la estabilidad de fluidos llaman semiconvección -(Grossman et al., 1993); (Kippenhahn and Weigert, 1990).

\subsection{Peso molecular medio y presión de radiación}

Para un gas ideal constituido de $n$ partículas por unidad de volumen y todas con peso molecular medio $\mu$, la ecuación de estado es: 


$$
P=n k T=\frac{\Re}{\mu} \varrho T
$$

con $\varrho=n \mu M_{u}$ ( $k$ : constante de Boltzman, $\Re=k / M_{u}$ : constante universal de los gases, $M_{u}$ : 1 uma). El peso molecular es una cantidad sin dimensiones y es simplemente la masa de la partícula dividida por 1 uma.

En los interiores estelares los gases están completamente ionizados. Tenemos por lo tanto una mezcla de 2 gases, el de los núcleos (el cual puede consistir de más de una componente) y el de los electrones libres. La mezcla puede ser tratada como un gas de una componente, si todas las componentes simples obedecen la ecuación del gas ideal.

Consideremos una mezcla de núcleos completamente ionizados. La composición química puede ser descripta especificando todos los $X_{i}$, la fracción de peso de los núcleos del tipo $i$, la cual tiene peso molecular $\mu_{i}$ y número de carga $Z_{i}$. Si tenemos $n_{i}$ núcleos por unidad de volumen y, densidad parcial $\varrho_{i}$, entonces es:

$$
X_{i}=\frac{\varrho_{i}}{\varrho}
$$

$\mathrm{y}$

$$
n_{i}=\frac{\varrho_{i}}{\mu_{i} M_{u}}=\frac{\varrho}{M_{u}} \frac{X_{i}}{\mu_{i}}
$$

(se ha despreciado la masa de los electrones comparada a la de los iones). La presión total $P$ de la mezcla, es la suma de las presiones parciales:

$$
P=P_{e}+\sum_{i} P_{i}=\left(n_{e}+\sum_{i} n_{i}\right) k T
$$

aquí $P_{e}$ es la presión de los electrones libres, mientras que $P_{i}$ es la presión parcial debida a los núcleos del tipo $i$. La contribución de un átomo completamente ionizado del elemento $i$ al número total de partículas (núcleo más $Z_{i}$ electrones libres) es $1+Z_{i}$; por lo tanto

$$
n=n_{e}+\sum_{i} n_{i}=\sum_{i}\left(1+Z_{i}\right) n_{i}
$$

y usando las ecs. (63) y (67) tenemos: 


$$
P=\sum_{i}\left(1+Z_{i}\right) n_{i} k T
$$

reemplazando la ec. (68) en la ec. (65)

$$
P=\sum_{i}\left(1+Z_{i}\right) \frac{\varrho_{i}}{\mu_{i} M_{u}} k T
$$

reemplazando en la ec. (69), la ec. (64):

$$
P=\sum_{i} \frac{\left(1+Z_{i}\right) X_{i}}{\mu_{i}} \frac{\varrho k T}{M_{u}}
$$

y como $\Re=k / M_{u}$ y por la ec. (63), obtenemos:

$$
P=\Re \sum_{i} \frac{\left(1+Z_{i}\right) X_{i}}{\mu_{i}} \varrho T=\frac{\Re}{\mu} \varrho T
$$

podemos por lo tanto escribir el peso molecular medio como:

$$
\mu=\left[\sum_{i} \frac{\left(1+Z_{i}\right) X_{i}}{\mu_{i}}\right]^{-1}
$$

por lo tanto introduciendo el peso molecular medio podemos tratar una mezcla de gases ideales como un gas ideal uniforme. Por ejemplo para el caso de hidrógeno puro (completamente ionizado) con $X_{H}=1, \mu_{H}=1, Z_{H}=1$ tenemos $\mu=1 / 2$, mientras que para un gas de helio completamente ionizado $\left(X_{H e}=1, \mu_{H e}=4, Z_{H e}=2\right)$ tenemos $\mu=4 / 3$. Para el caso de un gas neutro donde todos los electrones están en el átomo, se reemplaza $1+Z_{i}$ por $1:$

$$
\mu_{0}=\left[\sum_{i} \frac{X_{i}}{\mu_{i}}\right]^{-1}
$$

Para encontrar el peso molecular medio por electrón libre $\mu_{e}$, en un gas completamente ionizado, cada núcleo $i$ contribuye con $Z_{i}$ electrones libres, tenemos entonces:

$$
\mu_{e}=\left[\sum_{i} \frac{X_{i} Z_{i}}{\mu_{i}}\right]^{-1}
$$


Para los elementos más pesados que el helio es una buena aproximación tomar $\mu_{i} / Z_{i} \simeq 2$, encontrando:

$$
\mu_{e}=\left[X+\frac{1}{2} Y+\frac{1}{2}(1-X-Y)\right]^{-1}=\frac{2}{1+X}
$$

Pero la presión en una estrella no está sólo dada por la presión del gas, puesto que los fotones en el interior estelar pueden contribuir considerablemente a la presión. Ya que la radiación es prácticamente la del cuerpo negro, la presión de radiación $P_{r a d}$ está dada por:

$$
P_{\text {rad }}=\frac{1}{3} U=\frac{a}{3} T^{4}
$$

donde $U=a T^{4}$ según ec. (36). Entonces la presión $P$ consiste de la presión del gas $P_{\text {gas }} \mathrm{y}$ la presión de radiación $P_{r a d}$ :

$$
P=P_{g a s}+P_{r a d}=\frac{\Re}{\mu} \varrho T+\frac{a}{3} T^{4}
$$

donde hemos asumido que el gas es ideal. Definimos una cantidad útil, debido a la importancia de la presión de radiación la cual es:

$$
\beta=\frac{P_{g a s}}{P} \quad 1-\beta=\frac{P_{r a d}}{P}
$$

Para $\beta=1$, la presión de radiación es cero; mientras que si $\beta=0$, es la presión del gas cero. Las definiciones de la ec. (78) pueden ser usadas también si el gas es no ideal.

Otras dos cantidades útiles son las derivadas de la ec. (78):

$$
\begin{gathered}
\left(\frac{\partial \beta}{\partial T}\right)_{P}=-\left[\frac{\partial(1-\beta)}{\partial T}\right]_{P}=-\frac{4}{T}(1-\beta) \\
\left(\frac{\partial \beta}{\partial P}\right)_{T}=-\left[\frac{\partial(1-\beta)}{\partial P}\right]_{T}=\frac{1}{P}(1-\beta)
\end{gathered}
$$

\subsection{Física de las enanas blancas}

\subsubsection{Ecuación de estado para un gas ideal de electrones}

Para un sistema en equilibrio la función distribución sin dimensiones en el espacio de las fases, $f(\boldsymbol{x}, \boldsymbol{p})$ está dada por: 


$$
\frac{d n(\boldsymbol{x}, \boldsymbol{p})}{g d^{3} x d^{3} p / h^{3}}=f(\boldsymbol{x}, \boldsymbol{p})
$$

donde $d n$ es el número de electrones en el elemento de volumen del espacio de las fases $d^{3} x d^{3} p, g$ es el número de grados de libertad (o peso estadístico, para los electrones es $g=2$ ) y el denominador del miembro izquierdo de la ec. (81) representa el número de "celdas" correspondientes al volumen del espacio de las fases. La función $f$ da así el número de ocupación de una celda en el espacio de las fases. A partir de esta distribución pueden obtenerse todas las cantidades de interés (densidad numérica $n$, presión $P$ y densidad de energía $u$ ) empleando la relación relativista entre el momento y la energía cinética:

$$
\varepsilon(p)=m c^{2}\left[\sqrt{1+(p / m c)^{2}}-1\right]
$$

y la función distribución para un gas ideal de Fermi en equilibrio

$$
f(\varepsilon)=\frac{1}{\exp [(\varepsilon-\mu) / k T]+1}
$$

donde $k$ es la constante de Boltzmann y $\mu$ es el potencial químico. Para densidades bajas y altas temperaturas, $f(\varepsilon)$ se reduce a la distribución de Maxwell-Boltzmann. Para fermiones completamente degenerados $(T \rightarrow 0), \mu$ es llamada la energía de Fermi $\varepsilon_{F}$, y:

$$
f(\varepsilon)= \begin{cases}1 & \varepsilon \leq \varepsilon_{F} \\ 0 & \varepsilon>\varepsilon_{F}\end{cases}
$$

El impulso correspondiente a $\varepsilon_{F}$ de acuerdo a la ec. (82), se llama impulso de Fermi $p_{\mathrm{F}}$. La ecuación de estado puede obtenerse a partir de la función distribución en forma parametrizada como función de dos variables sin dimensiones: el "parámetro de degeneración" $\eta=\mu / k T$ y el "parámetro relativista" $\beta=k T / m c^{2}$. En término de estas variables:

$$
\begin{aligned}
& P=\frac{16 \pi \sqrt{2}}{3} \frac{m^{4} c^{5}}{h^{3}} \beta^{5 / 2}\left[F_{3 / 2}(\eta, \beta)+\frac{\beta}{2} F_{5 / 2}(\eta, \beta)\right] \\
& n=8 \pi \sqrt{2} \frac{m^{3} c^{3}}{h^{3}} \beta^{3 / 2}\left[F_{1 / 2}(\eta, \beta)+\beta F_{3 / 2}(\eta, \beta)\right] \\
& u=8 \pi \sqrt{2} \frac{m^{4} c^{5}}{h^{3}} \beta^{5 / 2}\left[F_{3 / 2}(\eta, \beta)+\beta F_{5 / 2}(\eta, \beta)\right]
\end{aligned}
$$

donde $P, n$ y $u$ son la presión, la densidad numérica y la densidad de energía. Las funciones $F_{K}$ aparecen definidas por las integrales: 


$$
F_{K}(\eta, \beta)=\int_{0}^{\infty} \frac{x^{K}(1+\beta x / 2)^{1 / 2}}{\exp (\eta+x)+1} d x
$$

y en general sólo pueden evaluarse numéricamente.

Las enanas blancas reales a baja degeneración siempre implican un régimen no-relativista, mientras que los efectos relativistas son sólo importantes para muy alta degeneración. La simplificación más significativa resulta de la aceptación de degeneración completa $(T \rightarrow 0)$, estas son las "estrellas de temperatura cero". Para este caso se utiliza $\eta \beta=\mu / m c^{2}$ con el límite $\varepsilon_{F} / m c^{2}$ para degeneración completa. Una variable más útil es el impulso de Fermi, una cantidad sin dimensiones es $x=p_{\mathrm{F}} / m c$ que se relaciona con $\eta \beta$ mediante la ec. (82):

$$
1+x^{2}=(\eta \beta)^{2}
$$

Para degeneración completa, la densidad numérica es:

$$
n=\frac{2}{h^{3}} \int_{0}^{p_{\mathrm{F}}} 4 \pi p^{2} d p=\frac{8 \pi}{3 h^{3}} p_{\mathrm{F}}{ }^{3}=\frac{8 \pi m^{3} c^{3}}{3 h^{3}} x^{3}=n_{0} x^{3}
$$

con

$$
x=p_{\mathrm{F}} / m c
$$

y la presión:

$$
P=\frac{8 \pi}{3 h^{3}} \int_{0}^{p_{\mathrm{F}}} \frac{\left(p^{4} / m\right) d p}{\sqrt{1+(p / m c)^{2}}}=\frac{\pi m^{4} c^{5}}{3 h^{3}} f(x)=P_{0} f(x)
$$

donde $f(x)$ está dada por:

$$
f(x)=x\left(x^{2}+1\right)^{1 / 2}\left(2 x^{2}-3\right)+3 \ln \left(x+\sqrt{1+x^{2}}\right)
$$

$P_{0}$ y $n_{0}$ involucran sólo las constantes físicas y $h$ enfatiza el rol de la mecánica cuántica en la estructura de las enanas blancas.

La densidad de materia $\varrho$ y la densidad numérica de electrones $n$, están relacionadas a través del requerimiento de la neutralidad de carga. En la materia completamente ionizada dados el peso molecular $\mu$ y la carga $Z$, entonces el número de iones es $n / Z$ y la densidad de masa es: 


$$
\varrho \simeq \varrho_{\text {iones }}=\frac{n}{Z} \mu M_{u}
$$

con $M_{u}$ la unidad de masa atómica, donde se ha despreciado la masa de los electrones frente a la masa de los iones. Por ec. (74) para una densidad uniforme, determinada por un peso molecular $\mu$ y una carga $Z$ (en ese caso la fracción de masa es $X=1$ ), tenemos

$$
\mu_{e} \simeq \frac{\mu}{Z}
$$

luego:

$$
\varrho \simeq n \mu_{e} M_{u}
$$

Reemplazando la ec. (90) obtenemos:

$$
\begin{aligned}
& \varrho=n_{0} \mu_{e} M_{u} x^{3}=\varrho_{0} \mu_{e} x^{3} \\
& \text { con } \quad \varrho_{0}=n_{0} M_{u}
\end{aligned}
$$

donde $\mu_{e}$ es el peso molecular por electrón, que toma el valor 2, para composiciones donde el número de neutrones $N$ es igual al de protones $Z$ (ya que $A \simeq \mu$ ). Para el hidrógeno vale 1 , para el hierro completamente ionizado vale 2.15. Si tenemos una mezcla de varios elementos debe tomarse el valor medio.

Tomando los casos límites $x \rightarrow 0$ (no-relativista) y $x \rightarrow \infty$ (ultra-relativista), en ambos casos los desarrollos de $f(x)$ permiten eliminar el "impulso de Fermi sin unidades, $x$ " resultando:

$$
\begin{aligned}
& P=\frac{8 P_{0}}{5 \varrho_{0}^{5 / 3}}\left(\frac{\varrho}{\mu_{e}}\right)^{5 / 3}=\left(\frac{3}{8 \pi}\right)^{2 / 3} \frac{h^{2}}{5 m M_{u}^{5 / 3}}\left(\frac{\varrho}{\mu_{e}}\right)^{5 / 3}, \quad x \ll 1 \\
& P=\frac{2 P_{0}}{\varrho_{0}^{4 / 3}}\left(\frac{\varrho}{\mu_{e}}\right)^{4 / 3}=\left(\frac{3}{8 \pi}\right)^{1 / 3} \frac{h c}{4 M_{u}^{4 / 3}}\left(\frac{\varrho}{\mu_{e}}\right)^{4 / 3}, \quad x \gg 1
\end{aligned}
$$




\subsubsection{La ecuación de Chandrasekhar}

Chandrasekhar (Chandrasekhar, 1939) demostró, que a partir del siguiente sistema de ecuaciones, compuesto por la ecuación de equilibrio hidrostático, distibución de masa y la ecuación de estado para un gas de electrones degenerados a temperatura cero:

$$
\frac{\partial P}{\partial r}=-\frac{G m}{r^{2}} \varrho \quad, \quad \frac{\partial m}{\partial r}=4 \pi r^{2} \varrho \quad, \quad P=P\left(\varrho, \mu_{e}\right)
$$

la estructura mecánica de una enana blanca puede derivarse a partir de la siguiente ecuación diferencial:

$$
\frac{1}{\eta^{2}} \frac{d}{d \eta}\left(\eta^{2} \frac{d \Phi}{d \eta}\right)=-\left(\Phi^{2}-\frac{1}{y_{0}^{2}}\right)^{3 / 2}
$$

conocida como ecuación de Chandrasekhar, con $\Phi(0)=1$ y $\left.\frac{d \Phi}{d \eta}\right|_{\eta=0}=0$. Para arribar a la ec. (102), se debe tener en cuenta la ecuación de estado para un gas totalmente degenerado relativista, dada por:

$$
\begin{aligned}
P & =A \cdot f(x) \\
A & =\frac{\pi m_{0}{ }^{4} c^{5}}{3 h^{3}} \\
f(x) & =x\left(2 x^{2}-3\right)\left(1+x^{2}\right)^{1 / 2}+3 \sinh ^{-1} x \\
x & =\frac{p_{\mathrm{F}}}{m_{0} c}
\end{aligned}
$$

donde $m_{0}$ : masa en reposo del electrón, $c$ : velocidad de la luz, $h$ : constante de Planck, $p_{\mathrm{F}}$ : impulso de Fermi -ver sec. (13.7.1)-, x: impulso de Fermi normalizado. Para este caso la densidad $\varrho$ está dada por:

$$
\begin{aligned}
\varrho & =B \cdot x^{3} \\
B & =\frac{8 \pi}{3}\left(\frac{m c}{h}\right)^{3} \mu_{e} M_{u}
\end{aligned}
$$

donde $\mu_{e}$ : es el peso molecular por electrón y $M_{u} 1$ uma. La variable $\eta$ está relacionada con el radio $r$ de la configuración mediante:

$$
\begin{aligned}
r & =\alpha \cdot \eta \\
\alpha & =\left(\frac{2 A}{\pi G}\right)^{\frac{1}{2}} \frac{1}{B y_{0}}
\end{aligned}
$$


donde $G$ : constante de gravitación. Como por definición $y=y_{0} \cdot \Phi$ con $y$ relacionado al impulso de Fermi mediante $y^{2}=x^{2}+1$, la cantidad $y_{0}$ es el valor de $y$ en el centro (ya que $\Phi(0)=1)$ y está vinculada a la densidad en el centro pues $\varrho_{0}=B\left(y_{0}{ }^{2}-1\right)^{3 / 2}$. Para el borde es $\varrho=0, x=0$, esto es $y=1$. Luego $1=y_{0} \cdot \Phi\left(\eta_{\mathrm{I}}\right)$, esto es $\Phi\left(\eta_{\mathrm{I}}\right)=y_{0}{ }^{-1}$ y $\eta_{\mathrm{I}}$ está vinculada al radio de la enana blanca mediante $R_{\mathrm{EB}}=\alpha \cdot \eta_{\mathrm{I}}$.

\subsubsection{Modelos estelares simples, relación masa-radio y el límite de Chandra- sekhar}

La estructura de las estrellas con simetría esférica está determinada por cuatro ecuaciones básicas, derivadas de las leyes de conservación de energía, masa y momento, y la ley que describe el transporte de energía en presencia de un gradiente de temperatura. Para estrellas a temperatura cero sólo son necesarias dos de estas ecuaciones, la ecuación de equilibrio hidrostático, ec. (28) y la ecuación de conservación de la masa, ec. (29)

$$
\frac{\partial P}{\partial r}=-\frac{G m}{r^{2}} \varrho \quad, \quad \frac{\partial m}{\partial r}=4 \pi r^{2} \varrho .
$$

El sistema de ecuaciones está completo con la ecuación de estado $P=P\left(\varrho, \mu_{e}\right)$. Las condiciones de borde son:

$$
\begin{array}{ll}
\text { en el centro: } & m(0)=0 \\
\text { en la superficie: } & P(R)=0
\end{array}
$$

Podemos entender las características básicas de la relación masa-radio sin resolver las tres ecuaciones anteriores con las dos condiciones de borde anteriores (se elimina $\varrho$ utilizando la ecuación de estado y puesto que $\mu_{e}$ es un valor preestablecido para el problema en cuestión, se resuelve un sistema de dos ecuaciones diferenciales en derivadas parciales de primer orden cuyas variables dependientes son $P$ y $m$, y la independiente es $r$ ) a partir de un simple análisis.

Si en la ec. (31) $\partial P / \partial m=-G m / 4 \pi r^{4}$, reemplazamos el miembro izquierdo por un gradiente de presión medio: $\left(P_{\text {sup }}-P_{\text {ctro }}\right) /\left(m_{\text {sup }}-m_{\text {ctro }}\right)$, donde

$$
\begin{aligned}
P_{\text {sup }} & =P(r=R)=0 \\
P_{\text {ctro }} & =P(r=0)=P_{c} \\
m_{\text {sup }} & =m(r=R)=M \\
m_{\text {ctro }} & =m(r=0)=0
\end{aligned}
$$


son los valores para la presión y la masa, en la superficie y en el centro. Y en el miembro derecho reemplazamos $m$ y $r$ por los valores medios $M / 2$ y $R / 2$, obteniendo:

$$
\frac{-P_{c}}{M} \cong-\frac{G M / 2}{4 \pi(R / 2)^{4}}
$$

dando así, un valor para la presión central

$$
P_{c} \cong \frac{2 G M^{2}}{\pi R^{4}}
$$

Análogamente para la ec. (29) $\partial m / \partial r=4 \pi r^{2} \varrho$, reemplazando el miembro izquierdo por un gradiente de masa medio $(M-0) /(R-0)$ y el derecho por los valores medios de la coordenada radio $r$ y la densidad $\varrho: \bar{r}=R / 2$ y $\varrho=[\varrho(r=R)+\varrho(r=0)] / 2=\varrho_{c} / 2$, donde $\varrho_{c}$ es el valor de la densidad central. Luego:

$$
\frac{M}{R} \cong 4 \pi\left(\frac{R}{2}\right)^{2} \frac{\varrho_{c}}{2}
$$

o lo que es lo mismo

$$
\varrho_{c} \cong \frac{2}{\pi} \frac{M}{R^{3}}
$$

arribando entonces a sendas condiciones centrales para la "presión gravitacional" y la densidad

$$
\begin{gathered}
P_{c} \propto \frac{G M^{2}}{R^{4}} \\
\varrho_{c} \propto \frac{M}{R^{3}}
\end{gathered}
$$

\section{Caso no-relativista}

Vimos que la presión electrónica para modelos a baja densidad, ec. $(99)(x \ll 1)$ es:

$$
P_{c} \propto \frac{P_{0}}{\varrho_{0}^{5 / 3}}\left(\frac{\varrho_{c}}{\mu_{e}}\right)^{5 / 3}
$$


donde el subíndice $c$ indica que está evaluada en el centro. Para $x \ll 1$ de ec. (97) es $\varrho \ll \varrho_{0} \mu_{e}$. Empleando la ec. (118) obtenemos:

$$
P_{c} \propto \frac{P_{0}}{\left(\varrho_{0} \mu_{e}\right)^{5 / 3}} \frac{M^{5 / 3}}{R^{5}}
$$

En equilibrio la presión gravitacional [ec. (117)] debe ser balanceada por la presión electrónica [ec. (120)] conduciendo a la relación masa-radio:

$$
\frac{G M^{2}}{R^{4}} \propto \frac{P_{0}}{\left(\varrho_{0} \mu_{e}\right)^{5 / 3}} \frac{M^{5 / 3}}{R^{5}}
$$

esto es:

$$
R \propto \frac{P_{0}}{G\left(\varrho_{0} \mu_{e}\right)^{5 / 3}} M^{-1 / 3}
$$

La ec. (121) muestra que en las enanas blancas para baja densidades, el radio decrece con el aumento de la masa como $M^{-1 / 3}$.

\section{Caso ultra-relativista}

En este caso ambas presiones dependen del radio de la misma forma, pues de ec. (100) $(x \gg 1)$, la presión en el centro es:

$$
P_{c} \propto \frac{P_{0}}{\varrho_{0}^{4 / 3}}\left(\frac{\varrho_{c}}{\mu_{e}}\right)^{4 / 3}
$$

Para $x \gg 1$ de ec. (97) es $\varrho \gg \varrho_{0} \mu_{e}$. Empleando la ec. (118) obtenemos:

$$
P_{c} \propto \frac{P_{0}}{\left(\varrho_{0} \mu_{e}\right)^{4 / 3}} \frac{M^{4 / 3}}{R^{4}}
$$

la cual tiene la misma dependencia con $R$ que la presión gravitacional. Igualando ambas presiones se obtiene:

$$
\frac{G M^{2}}{R^{4}} \propto \frac{P_{0}}{\left(\varrho_{0} \mu_{e}\right)^{4 / 3}} \frac{M^{4 / 3}}{R^{4}}
$$


dando:

$$
M \propto\left(\frac{P_{0}}{G}\right)^{3 / 2} \frac{1}{\left(\varrho_{0} \mu_{e}\right)^{2}}
$$

Esto es posible, entonces sólo para una única masa, la masa calculada por Chandrasekhar.

De ec. (92):

$$
P_{0} \propto \frac{m_{e}{ }^{4} c^{5}}{h^{3}}
$$

De ecs. (90) y (98)

$$
n_{0} \propto \frac{m_{e}{ }^{3} c^{3}}{h^{3}} \quad y \quad \varrho_{0}=n_{0} M_{u} \quad, \quad \varrho_{0} \propto \frac{m_{e}{ }^{3} c^{3}}{h^{3}} M_{u}
$$

Haciendo el cociente $P_{0}^{3 / 2} / \varrho_{0}^{2}$ obtenemos:

$$
\frac{P_{0}^{3 / 2}}{\varrho^{2}} \propto \frac{m_{e}{ }^{6} c^{15 / 2}}{h^{9 / 2}} \cdot \frac{h^{6}}{m_{e}{ }^{6} c^{6} M_{u}{ }^{2}}=\frac{(h c)^{3 / 2}}{M_{u}{ }^{2}}
$$

Reemplazando esto último en la ec. (124) obtenemos la masa de Chandrasekhar:

$$
M_{C h} \propto \frac{(h c)^{3 / 2}}{G^{3 / 2} M_{u}^{2}} \mu_{e}^{-2}
$$

Sin embargo esto no es real, debido a que la condición para la ecuación de estado ultra-relativista requiere una densidad infinita y $R=0$. La interpretación física es la siguiente: sólo para la masa $M=M_{C h}$ puede la presión gravitacional y la presión electrónica estar balanceadas. Para una masa levemente mayor, la presión gravitacional gana y la estrella colapsa dinámicamente a una singularidad. Para una masa levemente menor, la presión electrónica domina. La estrella comienza a expandirse, bajando la densidad y aproximándose a la ecuación de estado no-relativista en partes del interior estelar, hasta que el equilibrio puede obtenerse para un radio finito. Notar que la ec. (126) tampoco depende de la masa del electrón. 


\section{Apéndice B: Glosario}

AFTP (AGB Final Thermal Pulse): Pulso Térmico Final de la Rama Asintótica Gigante.

AGB (Asymptotic Giant Branch): Rama Asintótica Gigante.

AGB-manqué (manqué: del francés, ausente): significa que se halla ausente la Rama Asintótica Gigante. Son las estrellas que no alcanzan la fase de los pulsos térmicos.

born again: renacido; aunque ajusta mejor el término 'rejuvenecimiento' que es lo que sufre en realidad la estrella en su apariencia superficial.

CSPNe (Central Stars of Planetary Nebulae): Estrellas Centrales de las Nebulosas Planetarias.

dredge: dragar, rastrear. to dredge up: desenterrar. Se puede identificar como un 'mezclado convectivo externo' (MCE).

DA: Enanas blancas con líneas de H, sin HeI ni metales presentes.

DB: Enanas blancas con líneas de HeI, sin H ni metales presentes.

DC: Enanas blancas con espectro contínuo sin líneas distinguibles.

DO: Enanas blancas con líneas intensas HeII; puede haber HeI o H.

DQ: Enanas blancas con líneas de C.

DZ: Enanas blancas con líneas de metales, sin H ni He.

E-AGB (Early Asymptotic Giant Branch): Rama Asintótica Gigante Temprana.

EB: Enana Blanca.

EHB (Extreme Horizontal Branch): Rama Horizontal Extrema.

HBB (Hot Bottom Burning): Alude a la quema de combustible nuclear en la base caliente de la envoltura convectiva.

H-S: alude a la relación masa-radio de T. Hamada y E. E. Salpeter.

H-R: Hertzprung-Russell.

LTP (Late Thermal Pulse): Pulso Térmico Tardío. 
MCE: mezclado convectivo externo ver dredge up.

MLT: Mixing Length Theory.

OV (overshooting): Alude a una sobreextensión de la zona convectiva. Overshoot: irse por encima de; pasarse de.

PN (Planetary Nebula): Nebulosa Planetaria.

PNNV (Planetary Nebula Nucleus Variable): Núcleo de Nebulosa Planetaria Variable. PNNVs (Planetary Nebulae Nuclei Variables).

RGB (Red Giant Branch): Rama Gigante Roja.

VLTP (Very Late Thermal Pulse): Pulso Térmico Muy Tardío.

WC: estrellas Wolf-Rayet ricas en carbono.

WD (White Dwarf): estrellas enanas blancas.

WN: estrellas Wolf-Rayet ricas en nitrógeno.

WR: estrellas Wolf-Rayet.

ZAHB (Zero Age Horizontal Branch): Rama Horizontal de Edad Cero.

ZAMS (Zero Age Main Secuence): Secuencia Principal de Edad Cero. 


\section{Apéndice C: Tabla de símbolos}

- $L_{\odot}$ : luminosidades solares.

- $M_{\odot}$ : masas solares.

- $R_{\odot}$ : radios solares.

- $M_{*}$ : masa de la estrella.

- $R_{*}$ : radio de la estrella.

- $M_{u}$ : uma (unidad de masa atómica). 


\section{Apéndice D: Tabla de unidades}

- Velocidad de la luz en el vacío: $c=2.99792458 \times 10^{10} \mathrm{~cm} \mathrm{~s}^{-1}$

- Constante Newtoniana de la gravitación: $G=6.6726 \times 10^{-8} \mathrm{~g}^{-1} \mathrm{~cm}^{3} \mathrm{~s}^{-2}$

- Constante de Avogadro: $N_{A}=6.0221367 \times 10^{23} \mathrm{~mol}^{-1}$

- Constante de Planck: $h=6.626 \times 10^{-27}$ erg $s$

- Constante de Boltzmann: $k=1.380658 \times 10^{-16}$ erg $K^{-1}$

- Constante de densidad de radiación: $a=7.565 \times 10^{-15} \mathrm{erg} \mathrm{cm}^{-3} \mathrm{~K}^{-4}$

- Constante universal de los gases: $\Re=k / M_{u}=83145111.45 \mathrm{erg} K^{-1} g^{-1}$

- Conversión de eV a erg: $1 \mathrm{eV} \equiv 1.60217733 \times 10^{-12} \mathrm{erg}$

- Conversión de uma a gramo: 1 uma $\equiv 1.6605402 \times 10^{-24} g \equiv 1 M_{u}$

- Masa del electrón: $m_{e}=9.1093898 \times 10^{-28} \mathrm{~g} \equiv 5.4858 \times 10^{-4} u m a$ $\equiv 0.5109991 \mathrm{MeV} \mathrm{c}^{-2}$

- Masa del protón: $m_{\mathrm{H}}=m_{p}=1.6726231 \times 10^{-24} \mathrm{~g} \equiv 1.00727647 u m a$ $\equiv 938.27231 \mathrm{MeV} \mathrm{c}^{-2}$

- Masa del neutrón: $m_{n}=1.6749286 \times 10^{-24} \mathrm{~g} \equiv 1.0086649$ uma $\equiv 939.56563 \mathrm{MeV} \mathrm{c}^{-2}$

- Luminosidad solar: $L_{\odot}=(3.847 \pm 0.003) \times 10^{33} \operatorname{erg~s}^{-1}$

- Masa solar: $M_{\odot}=(1.9891 \pm 0.0004) \times 10^{33} \mathrm{~g}$

- Radio solar: $R_{\odot}=6.96 \times 10^{10} \mathrm{~cm}=6.96 \times 10^{5} \mathrm{~km}$

- Temperatura efectiva del Sol: $T_{\text {eff } \odot}=5780 \mathrm{~K}$

- $\operatorname{Parsec}(\mathrm{pc}): 1 p c \equiv 3.086 \times 10^{18} \mathrm{~cm} \equiv 3.261 \mathrm{AL}$

- Unidad Astronómica (UA): $1 U A \equiv 1.496 \times 10^{13} \mathrm{~cm}$ 


\section{Apéndice E: Abundancias Solares y Reacciones Nu- cleares}

\subsection{Abundancias solares}

La siguiente tabla fue obtenida a partir de (Arnett, 1996).

\begin{tabular}{|c|c|c|c|c|c|}
\hline Rango & $\mathrm{Z}$ & Símbolo & A & $\begin{array}{l}\text { Fracción } \\
\text { Nuclear }\end{array}$ & $\begin{array}{l}\text { Fuente } \\
\text { (proceso) }\end{array}$ \\
\hline 1 & 1 & $\mathrm{H}$ & 1 & $7.057 \mathrm{e}-01$ & Big Bang \\
\hline 2 & 2 & $\mathrm{He}$ & 4 & $2.752 \mathrm{e}-01$ & Big Bang, CNO, pp \\
\hline 3 & 8 & $\mathrm{O}$ & 16 & $9.592 \mathrm{e}-03$ & Helio \\
\hline 4 & 6 & $\mathrm{C}$ & 12 & $3.032 \mathrm{e}-03$ & Helio \\
\hline 5 & 10 & $\mathrm{Ne}$ & 20 & $1.548 \mathrm{e}-03$ & Carbono \\
\hline 6 & 26 & $\mathrm{Fe}$ & 56 & $1.169 \mathrm{e}-03$ & procesos-e \\
\hline 7 & 7 & $\mathrm{~N}$ & 14 & $1.105 \mathrm{e}-03$ & $\mathrm{CNO}$ \\
\hline 8 & 14 & $\mathrm{Si}$ & 28 & $6.530 \mathrm{e}-04$ & Oxígeno \\
\hline 9 & 12 & $\mathrm{Mg}$ & 24 & $5.130 \mathrm{e}-04$ & Carbono \\
\hline 10 & 16 & $\mathrm{~S}$ & 32 & $3.958 \mathrm{e}-04$ & Oxígeno \\
\hline 11 & 10 & $\mathrm{Ne}$ & 22 & $2.076 \mathrm{e}-04$ & Helio \\
\hline 12 & 12 & $\mathrm{Mg}$ & 26 & $7.892 \mathrm{e}-05$ & Carbono \\
\hline 13 & 18 & Ar & 36 & $7.740 \mathrm{e}-05$ & Silicio, Oxígeno \\
\hline 14 & 26 & $\mathrm{Fe}$ & 54 & $7.158 \mathrm{e}-05$ & procesos-e, Silicio \\
\hline 15 & 12 & $\mathrm{Mg}$ & 25 & $6.893 \mathrm{e}-05$ & Carbono \\
\hline 16 & 20 & $\mathrm{Ca}$ & 40 & $5.990 \mathrm{e}-05$ & Silicio, Oxígeno \\
\hline 17 & 13 & $\mathrm{Al}$ & 27 & $5.798 \mathrm{e}-05$ & Carbono \\
\hline 18 & 28 & $\mathrm{Ni}$ & 58 & $4.915 \mathrm{e}-05$ & Silicio, procesos-e \\
\hline 19 & 6 & $\mathrm{C}$ & 13 & $3.683 \mathrm{e}-05$ & $\mathrm{CNO}$ \\
\hline 20 & 2 & $\mathrm{He}$ & 3 & $3.453 \mathrm{e}-05$ & Big Bang, pp \\
\hline 21 & 14 & $\mathrm{Si}$ & 29 & $3.448 \mathrm{e}-05$ & Carbono, Neon \\
\hline 22 & 11 & $\mathrm{Na}$ & 23 & $3.339 \mathrm{e}-05$ & Carbono \\
\hline 23 & 26 & $\mathrm{Fe}$ & 57 & $2.840 \mathrm{e}-05$ & procesos-e \\
\hline 24 & 14 & $\mathrm{Si}$ & 30 & $2.345 \mathrm{e}-05$ & Carbono, Neon \\
\hline 25 & 1 & $\mathrm{H}$ & 2 & $2.317 \mathrm{e}-05$ & Big Bang \\
\hline
\end{tabular}




\subsection{Cadenas protón-protón}

Las cadenas protón-protón (Clayton, 1968).

\begin{tabular}{|c|c|c|}
\hline Reacción & $\mathrm{Q}[\mathrm{Mev}]$ & $\begin{array}{c}\epsilon_{\nu}[\mathrm{Mev}] \text { pérdida } \\
\text { por neutrinos }\end{array}$ \\
\hline \multicolumn{3}{|l|}{ PPI: } \\
\hline$p+p \rightarrow d+e^{+}+\nu$ & 1.442 & $\leq 0.420$ \\
\hline$d+p \rightarrow{ }^{3} \mathrm{He}+\gamma$ & 5.493 & \\
\hline${ }^{3} \mathrm{He}+{ }^{3} \mathrm{He} \rightarrow{ }^{4} \mathrm{He}+p+p$ & 12.859 & \\
\hline \multicolumn{3}{|l|}{ PPII: } \\
\hline$p+p \rightarrow d+e^{+}+\nu$ & 1.442 & $\leq 0.420$ \\
\hline$d+p \rightarrow{ }^{3} \mathrm{He}+\gamma$ & 5.493 & \\
\hline${ }^{3} \mathrm{He}+{ }^{4} \mathrm{He} \rightarrow{ }^{7} \mathrm{Be}+\gamma$ & 1.586 & \\
\hline${ }^{7} \mathrm{Be}+e^{-} \rightarrow{ }^{7} \mathrm{Li}+\nu$ & 0.861 & $\leq 0.861$ \\
\hline${ }^{7} \mathrm{Li}+p \rightarrow{ }^{4} \mathrm{He}+{ }^{4} \mathrm{He}$ & 17.347 & \\
\hline \multicolumn{3}{|l|}{ PPIII: } \\
\hline$p+p \rightarrow d+e^{+}+\nu$ & 1.442 & $\leq 0.420$ \\
\hline$d+p \rightarrow{ }^{3} \mathrm{He}+\gamma$ & 5.493 & \\
\hline${ }^{3} \mathrm{He}+{ }^{4} \mathrm{He} \rightarrow{ }^{7} \mathrm{Be}+\gamma$ & 1.586 & \\
\hline${ }^{7} \mathrm{Be}+p \rightarrow{ }^{8} \mathrm{~B}+\gamma$ & 0.135 & \\
\hline${ }^{8} \mathrm{~B} \rightarrow{ }^{8} \mathrm{Be}+e^{+}+\nu$ & & $\leq 15.000$ \\
\hline${ }^{8} \mathrm{Be} \rightarrow{ }^{4} \mathrm{He}+{ }^{4} \mathrm{He}$ & 18.074 & \\
\hline
\end{tabular}

$$
\begin{aligned}
& \varepsilon_{p p}=2.38 \times 10^{6} \psi f_{11} g_{11} \varrho X_{1}^{2} T_{6}^{-2 / 3} \exp \left(-33.80 / T_{6}^{1 / 3}\right) \\
& g_{11}=1+0.0123 T_{6}^{1 / 3}+0.0109 T_{6}^{2 / 3}+0.0009 T_{6}
\end{aligned}
$$

se ha tomado toda $\varepsilon_{p p}$ como proporcional a la rama $p p 1 ; \varepsilon_{p p}$ y $\varrho$ están en el cgs y $f_{11}$ es un factor de apantallamiento para esta reacción. El factor $\psi$ corrige por la generación de energía adicional en las ramas $p p 2$ y $p p 3$. La generación de energía por la cadena $p p$ ocurre para temperaturas $T_{6} \gtrsim 8$. 


\subsection{Ciclo CNOF}

Los ciclos carbono-nitrógeno-oxígeno-flúor (Arnett, 1996).

\begin{tabular}{|lcc|}
\hline \multicolumn{3}{|c|}{ Tabla 6: Los ciclos CNOF } \\
\hline \hline Reacción & Q[Mev] & $\begin{array}{c}\epsilon_{\nu}[\text { Mev }] \text { pérdida } \\
\text { por neutrinos }\end{array}$ \\
\hline $\mathrm{CN}:$ & & \\
${ }^{12} \mathrm{C}+p \rightarrow{ }^{13} \mathrm{~N}+\gamma$ & 1.944 & \\
${ }^{13} \mathrm{~N} \rightarrow{ }^{13} \mathrm{C}+e^{+}+\nu$ & 2.221 & $\leq 1.199$ \\
${ }^{13} \mathrm{C}+p \rightarrow{ }^{14} \mathrm{~N}+\gamma$ & 7.550 & \\
${ }^{14} \mathrm{~N}+p \rightarrow{ }^{15} \mathrm{O}+\gamma$ & 7.293 & \\
${ }^{15} \mathrm{O} \rightarrow{ }^{15} \mathrm{~N}+e^{+}+\nu$ & 2.761 & $\leq 1.732$ \\
${ }^{15} \mathrm{~N}+p \rightarrow{ }^{12} \mathrm{C}+{ }^{4} \mathrm{He}$ & 4.965 & \\
${ }^{15} \mathrm{~N}+p \rightarrow{ }^{16} \mathrm{O}+\gamma$ & 12.126 & \\
& & \\
$\mathrm{NO}:$ & 0.609 & \\
${ }^{16} \mathrm{O}+p \rightarrow{ }^{17} \mathrm{~F}+\gamma$ & & \\
${ }^{17} \mathrm{~F} \rightarrow{ }^{17} \mathrm{O}+e^{+}+\nu$ & 2.762 & $\leq .740$ \\
${ }^{17} \mathrm{O}+p \rightarrow{ }^{14} \mathrm{~N}+{ }^{4} \mathrm{He}$ & 1.193 & \\
$\mathrm{OF}:$ & & \\
${ }^{17} \mathrm{O}+p \rightarrow{ }^{18} \mathrm{~F}+\gamma$ & 5.601 & \\
${ }^{18} \mathrm{~F}+e^{-} \rightarrow{ }^{18} \mathrm{O}+\nu$ & 1.6555 & $\leq 0.6335$ \\
${ }^{18} \mathrm{O}+p \rightarrow{ }^{19} \mathrm{~F}+\gamma$ & 7.993 & \\
${ }^{19} \mathrm{~F}+p \rightarrow{ }^{16} \mathrm{O}+{ }^{4} \mathrm{He}$ & 8.115 & \\
\hline \hline
\end{tabular}

$$
\begin{aligned}
\varepsilon_{C N O} & =8.67 \times 10^{27} g_{14,1} X_{C N O} X_{1} \varrho T_{6}^{-2 / 3} \exp \left(-152.28 / T_{6}^{1 / 3}\right) \\
g_{14,1} & =1+0.0027 T_{6}^{1 / 3}-0.00778 T_{6}^{2 / 3}-0.000149 T_{6}
\end{aligned}
$$

donde $\varepsilon_{C N O}$ y $\varrho$ están en el cgs, $X_{C N O}$ es la suma de $X_{C}, X_{N}$ y $X_{O}$. La generación de energía por el ciclo $C N O$ ocurre para temperaturas $T_{6} \gtrsim 15$. 


\subsection{Quema de helio}

Ciclo triple alfa (Hansen and Kawaler, 1994).

\begin{tabular}{|lr|}
\hline \multicolumn{3}{|c|}{ Tabla 7: Ciclo 3- $\alpha$} \\
\hline \hline Reacción & Q[Mev] \\
\hline${ }^{4} \mathrm{He}+{ }^{4} \mathrm{He} \leftrightarrow{ }^{8} \mathrm{Be}$ & $-9.178 \mathrm{e}-2$ \\
${ }^{8} \mathrm{Be}+{ }^{4} \mathrm{He} \rightarrow{ }^{12} \mathrm{C}+\gamma$ & 7.367 \\
\hline \hline
\end{tabular}

$$
\varepsilon_{3 \alpha}=5.09 \times 10^{11} f_{3 \alpha} \varrho^{2} X_{4}^{3} T_{8}^{-3} \exp \left(-44.027 / T_{8}\right)
$$

$\varepsilon_{3 \alpha}$ y $\varrho$ están en el cgs y $f_{3 \alpha}$ es un factor de apantallamiento. La generación de energía por la reacción $3 \alpha$ ocurre para temperaturas $T_{6} \gtrsim 100$.

Algunas reacciones mediante las cuales se capturan partículas $\alpha$ (Arnett, 1996).

\begin{tabular}{|lc|}
\hline \multicolumn{3}{|c|}{ Tabla 8: Quema de helio } \\
\hline \hline Reacción & Q[Mev] \\
\hline${ }^{12} \mathrm{C}+{ }^{4} \mathrm{He} \rightarrow{ }^{16} \mathrm{O}+\gamma$ & 7.162 \\
${ }^{13} \mathrm{C}+{ }^{4} \mathrm{He} \rightarrow{ }^{16} \mathrm{O}+n$ & \\
${ }^{14} \mathrm{~N}+{ }^{4} \mathrm{He} \rightarrow{ }^{18} \mathrm{O}$ & \\
${ }^{14} \mathrm{~N}+{ }^{4} \mathrm{He} \rightarrow{ }^{18} \mathrm{~F}+\gamma$ & \\
${ }^{15} \mathrm{~N}+{ }^{4} \mathrm{He} \rightarrow{ }^{19} \mathrm{~F}+\gamma$ & \\
${ }^{16} \mathrm{O}+{ }^{4} \mathrm{He} \rightarrow{ }^{20} \mathrm{Ne}+\gamma$ & 4.730 \\
${ }^{17} \mathrm{O}+{ }^{4} \mathrm{He} \rightarrow{ }^{20} \mathrm{Ne}+p$ & \\
${ }^{18} \mathrm{O}+{ }^{4} \mathrm{He} \rightarrow{ }^{22} \mathrm{Ne}+\gamma$ & \\
${ }^{20} \mathrm{Ne}+{ }^{4} \mathrm{He} \rightarrow{ }^{24} \mathrm{Mg}+\gamma$ & 9.310 \\
${ }^{22} \mathrm{Ne}+{ }^{4} \mathrm{He} \rightarrow{ }^{25} \mathrm{Mg}+n$ & \\
${ }^{22} \mathrm{Ne}+{ }^{4} \mathrm{He} \rightarrow{ }^{26} \mathrm{Mg}+\gamma$ & \\
\hline \hline
\end{tabular}

$$
\begin{aligned}
\varepsilon_{12, \alpha}= & 1.3 \times 10^{27} f_{12,4} X_{12} X_{4} \varrho T_{8}^{-2}\left(\frac{1+0.134 T_{8}^{2 / 3}}{1+0.01 T_{8}^{2 / 3}}\right) \exp \left(-69.20 / T_{8}^{1 / 3}\right) \\
\varepsilon_{16, \alpha} \approx & X_{16} X_{4} \varrho f_{16,4}\left[1.82 \times 10^{27} T_{8}^{-2 / 3} \exp \left(-85.68 / T_{8}^{1 / 3}\right)\right. \\
& \left.+9.22 \times 10^{19} T_{8}^{-3 / 2} \exp \left(-103.59 / T_{8}\right)\right]
\end{aligned}
$$




\subsection{Quema de carbono y oxígeno}

Carbono (Clayton, 1968).

\begin{tabular}{|lr|}
\hline \multicolumn{2}{|c|}{ Tabla 9: Quema de carbono } \\
\hline \hline Reacción & Q[Mev] \\
\hline${ }^{12} \mathrm{C}+{ }^{12} \mathrm{C} \rightarrow{ }^{24} \mathrm{Mg}+\gamma$ & 13.930 \\
${ }^{12} \mathrm{C}+{ }^{12} \mathrm{C} \rightarrow{ }^{23} \mathrm{Na}+p$ & 2.238 \\
${ }^{12} \mathrm{C}+{ }^{12} \mathrm{C} \rightarrow{ }^{20} \mathrm{Ne}+{ }^{4} \mathrm{He}$ & 4.616 \\
${ }^{12} \mathrm{C}+{ }^{12} \mathrm{C} \rightarrow{ }^{23} \mathrm{Mg}+n$ & -2.605 \\
${ }^{12} \mathrm{C}+{ }^{12} \mathrm{C} \rightarrow{ }^{16} \mathrm{O}+{ }^{4} \mathrm{He}+{ }^{4} \mathrm{He}$ & -0.114 \\
\hline \hline
\end{tabular}

$$
\begin{aligned}
\varepsilon_{C C} \approx & 5.49 \times 10^{43} f_{C C} \varrho X_{12}^{2} T_{9}^{-3 / 2} T_{9 a}^{5 / 6} \exp \left(-84.165 / T_{9 a}^{1 / 3}\right) \\
& \times\left[\exp \left(-0.01 / T_{9 a}^{4}\right)+5.56 \times 10^{-3} \exp \left(1.685 T_{9 a}^{2 / 3}\right)\right]^{-1} \\
T_{9 a}= & T_{9} /\left(1+0.067 T_{9}\right)
\end{aligned}
$$

La generación de energía por la reacción $C-C$ ocurre para temperaturas $T_{6} \gtrsim 500$. Oxígeno (Clayton, 1968).

Tabla 10: Quema del oxígeno

\begin{tabular}{lr}
\hline \hline Reacción & Q[Mev] \\
\hline${ }^{16} \mathrm{O}+{ }^{16} \mathrm{O} \rightarrow{ }^{32} \mathrm{~S}+\gamma$ & 16.539 \\
${ }^{16} \mathrm{O}+{ }^{16} \mathrm{O} \rightarrow{ }^{31} \mathrm{P}+p$ & 7.676 \\
${ }^{16} \mathrm{O}+{ }^{16} \mathrm{O} \rightarrow{ }^{31} \mathrm{~S}+n$ & 1.459 \\
${ }^{16} \mathrm{O}+{ }^{16} \mathrm{O} \rightarrow{ }^{28} \mathrm{Si}+{ }^{4} \mathrm{He}$ & 9.593 \\
${ }^{16} \mathrm{O}+{ }^{16} \mathrm{O} \rightarrow{ }^{24} \mathrm{Mg}+{ }^{4} \mathrm{He}+{ }^{4} \mathrm{He}$ & -0.393 \\
\hline \hline
\end{tabular}

$$
\begin{aligned}
\varepsilon_{O O} \approx & 1.09 \times 10^{54} f_{O O} \varrho X_{16}^{2} T_{9}^{-3 / 2} T_{9 a}^{5 / 6} \exp \left(-135.93 / T_{9 a}^{1 / 3}\right) \\
& \times\left[\exp \left(-0.032 / T_{9 a}^{4}\right)+3.89 \times 10^{-4} \exp \left(2.659 T_{9 a}^{-2 / 3}\right)\right]^{-1} \\
T_{9 a}= & T_{9} /\left(1+0.067 T_{9}\right)
\end{aligned}
$$

La generación de energía por la reacción $O-O$ ocurre para temperaturas $T_{6} \gtrsim 1000$. 


\subsection{Fotodesintegración del neón}

Neón (Clayton, 1968).

\begin{tabular}{lr}
\multicolumn{3}{|l}{ Tabla 11: Fotodesintegración del neón } \\
\hline \hline Reacción & Q $[$ Mev] \\
\hline${ }^{20} \mathrm{Ne}+\gamma \rightarrow{ }^{16} \mathrm{O}+{ }^{4} \mathrm{He}$ & -4.73 \\
\hline \hline
\end{tabular}

La fotodesintegración del neón ocurre antes de la quema de oxígeno. 


\section{Bibliografía}

Alexander, D. R. and Ferguson, J. W.: 1994, ApJ 437, 879

Althaus, L. G.: 1996, Ph.D. thesis, Facultad de Ciencias Astronómicas y Geofísicas, Universidad Nacional de La Plata

Althaus, L. G. and Benvenuto, O. G.: 2000, MNRAS 317, 952

Althaus, L. G. and Córsico, A. H.: 2004, A\&A 417, 1115

Althaus, L. G., Serenelli, A. M., Córsico, A. H., and Montgomery, M. H.: 2003, A\& A 404, 593

Angulo, C.: 1999, Nucl. Phys. A 656, 3

Arnett, D.: 1996, Supernovae and Nucleosynthesis: an investigation of the history of matter, from the big bang to the present, Princeton University Press, Princeton, USA

Arnett, W. D. and Truran, J. W.: 1969, ApJ 157, 339

Asplund, M., Lambert, D. L., Kipper, T., Pollacco, D., and Shetrone, M. D.: 1999, A\&A 343, 507

Beauchamp, A., Wesemael, F., Bergeron, P., Fontaine, G., Saffer, R. A., Liebert, J., and Brassard, P.: 1999, ApJ 516, 887

Blöcker, T.: 1995a, A\&A 299, 755

Blöcker, T.: 1995b, A\&A 297, 727

Blöcker, T.: 2001, ApSS 275, 1

Blöcker, T. and Schönberner, D.: 1997, A\&A 324, 991

Boothroyd, A. D., Sackmann, I. J., and Ahern, S. C.: 1993, ApJ 416, 762

Bradley, P. A.: 2000, Baltic Astonomy 9, 485

Bradley, P. A. and Winget, D. E.: 1994a, ApJ 421, 236

Bradley, P. A. and Winget, D. E.: 1994b, ApJ 430, 850

Burgers, J. M.: 1969, Flow Equations for Composite Gases, Academic, New York

Caughlan, G. R. and Fowler, W. A.: 1988, Atomic Data and Nuclear Data Tables 40, 290

Chandrasekhar, S.: 1935, MNRAS 95, 207

Chandrasekhar, S.: 1939, An Introduction to the Study of Stellar Structure, Princeton Series in Astrophysics, University of Chicago Press, Chicago

Christensen-Dalsgaard, J.: 1998, in H. Kjeldsen and T. R. Bedding (eds.), The First MONS Workshop: Science With a Small Space Telescope, Aarhus Universitet

Clayton, D. D.: 1968, Principles of Stellar Evolution and Nucleosynthesis, University of Chicago Press, 1983 edition

Córsico, A. H.: 2003, Ph.D. thesis, Facultad de Ciencias Astronómicas y Geofísicas, Universidad Nacional de La Plata

Córsico, A. H., Althaus, L. G., Montgomery, M. H., García-Berro, E., and Isern, J.: 2004, 


\section{$A \& A$ submitted}

D'Antona, F. and Mazzitelli, I.: 1996, ApJ 470, 1093

Dehner, B. T. and Kawaler, S. D.: 1995, ApJ 445, L141

Dreizler, S. and Heber, U.: 1998, A\&A 334, 618

Dreizler, S., Werner, K., and Heber, U.: 1995, in D. Koester and K. Werner (eds.), White

Dwarfs, Vol. 443 of Lecture Notes in Physics, p. 160, Springer, Berlin

Duerbeck, H. W., Benetti, S., Gautschy, A., van Genderen C. Kemper, A. M., Lillier, W., and Thomas, T.: 1997, AJ 114, 1657

Duerbeck, H. W., Liller, W., and Benetti, S.: 2000, AJ 119, 2360

Fermi, E.: 1936, Thermodynamics, EUDEBA, $19855^{\text {th }}$ edition

Fontaine, G. and Brassard, P.: 2002, ApJ 581, L33

Ford, H. C. and Jacoby, G.: 1983, ApJ 266(1), 298

Forestini, M. and Charbonnel, C.: 1997, A\&AS 123, 241

Freytag, B., Ludwig, H.-G., and Steffen, M.: 1996, A\&A 313, 497

Frost, C., Cannon, R., Lattanzio, J., Wood, P. R., and Forestini, M.: 1998, A\& A 332, L17

Fujimoto, M. Y.: 1977, PASJ 29, 331

Gautschy, A. and Althaus, L. G.: 2002, A\&A 382, 141

González, G., Lambert, D. L., Wallerstein, G., Rao, N. K., Smith, V. V., and McCarthy,

J. K.: 1998, ApJS 114, 133

Green, R. F., Schmidt, M., and Liebert, J.: 1986, ApJS 61, 305

Grossman, S. A., Narayan, R., and Arnett, D.: 1993, ApJ 407, 284

Grossman, S. A. and Taam, R. E.: 1996, MNRAS 283, 1165

Habing, H. J.: 1996, A\&AR 7, 97

Hamada, T. and Salpeter, E. E.: 1961, ApJ 134, 683

Hansen, C. J. and Kawaler, S. D.: 1994, Stellar Interiors: Physical Principles, Structure and Evolution, A\&A Library, Springer-Verlag New York, $19993^{\text {rd }}$ edition

Hazard, C., Terlevich, B., Morton, D. C., Sargent, W. L. W., and Ferland, G.: 1980, Nature 285, 463

Herwig, F.: 2000, A\&A 360, 952

Herwig, F.: 2001a, ApSS 275, 100

Herwig, F.: 2001b, ApJ 554, L71

Herwig, F.: 2003, in M. D. S. Kwok and R. Sutherland (eds.), Planetary Nebulae: their evolution and role in the Universe, Vol. 209 of IAU Symp., p. 111, ASP

Herwig, F., Blöcker, T., Langer, N., and Driebe, T.: 1999a, A\&A 349, L5

Herwig, F., Blöcker, T., and Schönberner, D.: 1999b, in T. L. Bertre, A. Lebre, and C.

Waelkens (eds.), AGB Stars, Vol. 191 of IAU Symp., p. 41, ASP

Herwig, F., Blöcker, T., Schönberner, D., and Eid, M. E.: 1997, A\&AA 324, L81 
Iben, I. J.: 1975, ApJ 196, 525

Iben, I. J.: 1976, ApJ 208, 165

Iben, I. J.: 1981, ApJ 246, 278

Iben, I. J.: 1984, ApJ 277, 333

Iben, I. J.: 1995, Phys. Rep. 250, 1

Iben, I. J., Kaler, J. B., Truran, J. W., and Renzini, A.: 1983, ApJ 264, 605

Iben, I. J. and MacDonald, J.: 1985, ApJ 296, 540

Iben, I. J. and MacDonald, J.: 1995, in D. Koester and K. Werner (eds.), White dwarfs, Vol. 443 of Lecture Notes in Physics, p. 48, Springer, Berlin

Iben, I. J. and Renzini, A.: 1983, Ann. Rev. A\&A 21, 271

Iben, I. J., Tutukov, A. V., and Yungelson, L. R.: 1996, ApJ 456, 750

Iglesias, C. A. and Rogers, F. J.: 1996, ApJ 464, 943

Isern, J., Canal, R., and Labay, J.: 1991, ApJ 372, L83

Itoh, N., Kohyama, Y., Matsumoto, N., and Seki, M.: 1984a, ApJ 285, 758

Itoh, N., Kohyama, Y., Matsumoto, N., and Seki, M.: 1984b, ApJ 285, 304

Itoh, N., Kohyama, Y., Matsumoto, N., and Seki, M.: 1987, ApJ 322, 584, (erratum)

Kippenhahn, R. and Weigert, A.: 1990, Stellar Structure and Evolution, A\&A Library, Springer-Verlag Berlin Heidelberg, $19912^{\text {nd }}$ edition

Kippenhahn, R., Weigert, A., and Hofmeister, E.: 1967, in S. Fernbach and M. Rottenberg (eds.), Methods in computational Physics, No. 7 in Alder B, p. 129, New York: Academic Press

Kipper, T.: 1996, in C. S. Jefferey and U. Heber (eds.), Hydrogen-deficient stars, Vol. 96 of ASP Conf. Ser., p. 329

Koesterke, L. and Hamann, W. R.: 1997, A\&A 320, 91

Langer, N., Eid, M. E., and Fricke, K. J.: 1985, A\&A 145, 179

Lawlor, T. M. and MacDonald, J.: 2003, ApJ 583, 913

Ledoux, P.: 1947, ApJ 105, 305

Madej, J., , Należyty, M., and Althaus, L. G.: 2004, A\&A aceptado, Letter

Madej, J., Pietrachowicz, M., Joss, P. C., Majczyna, A., Różańska, A., and Należyty, M.: 2003, AधA 412, 837

Magni, G. and Mazzitelli, I.: 1979, A\&A 72, 134

Mazzitelli, I., D’Antona, F., and Ventura, P.: 1999, A\&A 348, 846

Panei, J. A., Althaus, L. G., and Benvenuto, O. G.: 2000a, MNRAS 312, 531

Panei, J. A., Althaus, L. G., and Benvenuto, O. G.: 2000b, A\&A 353, 970

Pauldrach, A., Puls, J., Kudritzki, R. P., Méndez, R., and Heap, S. R.: 1988, A\&A 207, 123

Perinotto, M.: 1989, in S. Torres-Peimbert (ed.), Planetary Nebulae, Vol. 131 of IAU Symp., 
p. 293, Reidel, Dordrecht

Pollacco, D.: 1999, MNRAS 304, 127

Provencal, J. L., Shipman, H. L., Hog, E., and Thejll, P.: 1998, ApJ 494, 759

Salpeter, E. E.: 1961, ApJ 134, 669

Scalo, J. M., Despain, K. H., and Ulrich, R. K.: 1975, ApJ 196, 805

Schönberner, D.: 1979, A\&A 79, 108

Schönberner, D.: 1983, ApJ 272, 708

Schönberner, D. and Blöcker, T.: 1992, in U. Heber and C. S. Jeffery (eds.), Atmospheres of Early-Type Stars, p. 305, Springer

Schönberner, D. and Blöcker, T.: 1993, in D. Sasselov (ed.), Luminous High Latitude Stars, Vol. 45, p. 337, ASP Conf. Ser.

Schönberner, D. and Blöcker, T.: 1996, ApSS 245, 201

Schwarzschild, M. and Härm, R.: 1965, ApJ 142, 855

Serenelli, A. M.: 2002, Ph.D. thesis, Facultad de Ciencias Astronómicas y Geofísicas, Universidad Nacional de La Plata

Silvotti, R., Dreizler, S., Handler, G., and Jiang, X. J.: 1999, A\&A 342, 745

Straniero, O., Domínguez, I., Imbriani, G., and Piersanti, L.: 2003, ApJ 583, 878

Tassoul, M., Fontaine, G., and Winget, D. E.: 1990, ApJS 72, 335

Unglaub, K. and Bues, I.: 2000, A\&A 359, 1042

Unno, W., Osaki, Y., Ando, H., Saio, H., and Shibahashi, H.: 1989, Nonradial Oscillations of Stars, University of Tokyo Press, $2^{\text {nd }}$ edition

van Loon, J. T., Zijlstra, A. A., and Groenewegen, M. A. T.: 1999, A\&A 346, 805

Vassiliadis, E. and Wood, P. R.: 1993a, ApJ 92, 125

Vassiliadis, E. and Wood, P. R.: 1993b, ApJS 413, 641

Ventura, P., D'Antona, F., and Mazzitelli, I.: 1999, ApJ 524, L111

Weigert, A.: 1966, Z. Astrophys. 64, 395

Werner, K.: 1992, in The Atmospheres of Early-Type Stars, Vol. 401 of Lecture Notes in Physics, p. 273, Springer, Berlin

Werner, K.: 1993, in White Dwarfs: Advances in Observation and Theory, Vol. 403 of NATO $A S I C$ Proc, Springer, Berlin

Werner, K.: 2001, ApSS 275, 27

Werner, K., Dreizler, S., Heber, U., Rauch, T., Wisotzki, L., and Hagen, H.-J.: 1995, A\&̈A 293, L75

Winget, D. E., Robinson, E. L., Nather, R. D., and Fontaine, G.: 1982a, ApJ 262, L11

Winget, D. E., van Horn, H. M., Tassoul, M., Fontaine, G., Hansen, C. J., and Carroll, B. W.: 1982b, ApJ 252, L65

Winters, J.-M.: 1998, ApSS 255, 257 
Wood, P. R.: 1981, in I. Iben and A. Renzini (eds.), Physical Processes in Red Giants, p. 135, Reidel, Dordrecht

Wood, P. R.: 1997, in H. J. Habing and H. J. G. L. M. Lamers (eds.), Planetary Nebulae, Vol. 180 of IAU Symp., p. 297, Kluwer, Dordrecht 


\section{Tesis Doctoral}

Formación y Evolución de

Enanas Blancas

Deficientes en Hidrógeno

Día: $1^{\circ}$ de Julio de 2004

Hora: 11:00

Salón Meridiano 